\title{
Journal of Cardiology and Therapy
}

\section{Possible Pivotal Role of Latent Chronic T. gondii Infection in the Pathogenesis of Atherosclerosis}

\author{
Joseph Prandota
}

Joseph Prandota, Faculty of Health Sciences, Wroclaw Medical University, Wroclaw, Poland

Conflict-of-interest statement: The author(s) declare(s) that there is no conflict of interest regarding the publication of this paper.

Open-Access: This article is an open-access article which was selected by an in-house editor and fully peer-reviewed by external reviewers. It is distributed in accordance with the Creative Commons Attribution Non Commercial (CC BY-NC 4.0) license, which permits others to distribute, remix, adapt, build upon this work noncommercially, and license their derivative works on different terms, provided the original work is properly cited and the use is noncommercial. See: http: //creativecommons.org/licenses/by-nc/4.0/

Correspondence to: Joseph Prandota, Faculty of Health Sciences, Wroclaw Medical University, 5 Bartla Street, 51-618 Wroclaw, Poland.

Email: jozef.prandota9@gmail.com

Telephone: $+48-713484210$

Fax: $+48-713432086$

Received: November 30, 2016

Revised: February 12, 2017

Accepted: February 14, 2017

Published online: April 17, 2017

\section{ABSTRACT}

Cardiovascular diseases such as atherosclerosis, atherothrombosis, coronary artery disease and stroke are the main causes of death worldwide. Atherosclerosis is a chronic, progressive immuneinflammatory and fibroproliferative disease of medium and large sized arteries with increased blood lipoprotein/cholesterol and their disposition in the arterial wall, important $\mathrm{T}_{\mathrm{H}} 1$ type proinflammatory reaction, and thrombogenic status. Elevated plasma levels of proinflammatory cytokines have been demonstrated in patients with established atherosclerotic disease, and therefore it is believed that cytokines are key players in all stages of disease and have a profound influence on the pathogenesis of the disease. Several authors suggested that the number of different pathogens, including T. gondii, may promote synergistic inflammatory responses that are capable of triggering and exacerbating atherosclerotic process. Latent chronic
T. gondii infection may be a frequent cause of vascular endothelial cell dysfunction because this pathogen attacks all nucleated cells, and endothelium cells have enhanced susceptibility to infection with $T$. gondii tachyzoites. Oxidative stress characteristic for atherosclerosis may be caused by the host infection with the parasite. T. gondii is unable to synthesize sterol and acquires cholesterol from the host LDL receptor pathway. The accumulation of foamy transformed macrophages in the aortic intima characteristic for atherosclerotic lesion is due to acyl-CoA: cholesterol acyltransferase 1 and 2 (ACAT1 and ACAT2) increased expression in macrophages and other cells infected with $T$. gondii. ACAT2 is crucial in foam cell formation and development of atherosclerosis. The parasite also expresses two cholesteryl ester(CE)-synthesizing enzymes $\mathrm{TgACAT} 1$ and $\operatorname{TgACAT} 2$ that contribute to the CEs formation for storage in lipid bodies, but at the same time ACAT and CEs play a crucial role in replication of the pathogen. It must be emphasized that the increased expression of ACAT1 and ACAT2 normally present in macrophages may thus be further enhanced by the superimposing $\operatorname{TgACAT} 1$ and $T g$ ACAT2 activities available in the host cells infected with $T$. gondii. In addition, proinflammatory cytokines increase foam cell formation and latent chronic T. gondii infection persistently generates these biomediators and thus play an important role in foam cell biogenesis. Patients with atherosclerosis had increased plasma levels of TGF- $\beta$ and this cytokine increased $T$. gondii replication in the host cells, and participated in development of fibrotic changes in atherosclerotic lesions. The parasite may also produce some extracellular vesicles known to play a role in triggering cardiovascular events. Atherogenesis involved platelets activation with subsequent serotonin release. Hyperserotoninemia was also reported in autistic and mentally retarded children, and recently, a significantly higher seroprevalence of chronic toxoplasmosis was found in autistic children as compared with controls. T. gondii infection caused also increased leptin levels, and a marked association between $T$. gondii seropositivity and obesity has been demonstrated. Cysteine cathepsins (Cat) also play an important role of in foam cell formation and generation of amyloid in atherosclerotic arteries. Infection of vascular endothelial cells with $T$. gondii tachyzoites provide an additional source of Cat that are superimposing on similar enzymes already present in host cells, because the parasite expresses few members of the cathepsins, such as CatL-like, CatB-like, and CatClike proteases. Finally, vitamin D exerted beneficial effects in both atherosclerosis and $T$. gondii infection, especially that it improved host immunity and decreased proliferation of $T$. gondii tachyzoites in 
macrophages and reduced tissue pathology caused by the pathogen. Because the parasite is widely disseminated in animals and humans, it seems that latent chronic toxoplasmosis play a critical role in the pathogenesis of atherosclerosis.

Key words: Atherosclerosis; Atherothrombosis; Cardiovascular diseases; Stroke; T. gondii Infection; Endothelial dysfunction; Oxidative stress; ACAT1; ACAT2; Foam cells; Mast cells; Blood coagulation; Iron; Leptin; Extracellular vesicles; Platelets; Serotonin; Cysteine cathepsins; Vitamin D; Statins; Propranolol

(C) 2017 The Author(s). Published by ACT Publishing Group Ltd. All rights reserved.

Prandota J. Possible Pivotal Role of Latent Chronic T. gondii Infection in the Pathogenesis of Atherosclerosis. Journal of Cardiology and Therapy 2016; 4(2): 611-663 Available from: URL: http: //www.ghrnet.org/index.php/jct/article/view/1939

\section{INTRODUCTION}

\section{Atherosclerosis}

Cardiovascular diseases (CVDs) are the leading cause of death worldwide, accounting approximately 17.3 million deaths per year ${ }^{[1,2]}$, and this number will probably increase to 23.3 million by $2030^{[3]}$. Atherosclerosis also is the main cause of death, and in Europe, cardiovascular disease, atherothrombosis, coronary artery disease and stroke account for over 4 million deaths per year ${ }^{[4]}$.

Atherosclerosis is a chronic, progressive immune-inflammatory and fibroproliferative disease of medium and large sized arteries with three main components: (a) increased blood lipoprotein/ cholesterol and their disposition in the arterial wall; (b) an important $\mathrm{T}_{\mathrm{H}} 1$ type proinflammatory reaction; and (c) thrombogenic status ${ }^{[5]}$. The development of atheromatous plaques involves a number of cellular and molecular events including endothelial dysfunction, leukocyte recruitment and diapedesis, low-density lipoprotein (LDL) transcytosis and oxidation, intimal smooth muscle cells recruitment and proliferation, synthesis of extracellular matrix proteins, and accumulation of foamy macrophages along with interplay of various cytokines ${ }^{[6]}$.

Atherosclerotic process is initiated by the infiltration, retention and accumulation of LDL in the tunica intima of the arterial wall ${ }^{[7-9]}$, and typically begins with endothelial cell dysfunction, mononuclear cell recruitment into vascular wall, and the differentiation of macrophages into foam cells $\mathrm{s}^{[10,11]}$. Atherosclerotic lesions are characterized by progressive accumulation of lipids, macrophages, NK cells, T and B lymphocytes, smooth muscle cells, and fibroproliferative elements in the intima of arteries ${ }^{[12,13]}$. Functional analysis of different T-cell subsets role identified the $\mathrm{T}_{\mathrm{H}} 1$ responses as proatherogenic, whereas $T_{H} 2$ immune responses exert antiatherogenic actions ${ }^{[14,15]}$. Atherosclerosis is also associated with B-cell activation, and traditional $\mathrm{T}$ cell-driven $\mathrm{B} 2$ cells responses promote atherosclerosis, while innate $\mathrm{B} 1$ cells provide protective action through the secretion of naturally occurring antibodies ${ }^{[14,15]}$.

Several authors have hypothesized that the number of different pathogens, including $T$. gondii ${ }^{[16]}$, may promote synergistic inflammatory responses that are capable of triggering and exacerbating atherosclerosis ${ }^{[17]}$. Lipopolysaccharide was found to be a potential contributor to the development of atherosclerotic plaque even at extremely low serum concentrations ${ }^{[18,19]}$. In susceptible individuals, the presence of a chronic infective stimulus, as well as a prolific generation of proinflammatory cytokines, may directly activate metabolic pathways, such as for example cholesterol esterification, leading to foam cell formation and development of atherosclerosis ${ }^{[20]}$. Macrophages and foam cells present in the evolving atherosclerotic lesion, secrete various proinflammatory cytokines, such as TNF- $\alpha$, IFN- $\gamma$, IL-6, and chemokines, including ICAM-1 and VCAM-1. In response to enhanced production of proinflammatory cytokines, endothelial cells express high levels of leukocyte adhesion molecules on their surface, leading to further mononuclear cell recruitment and, hence, to development of a chronic inflammatory state ${ }^{[21-23]}$.

\section{T. gondii infection}

Toxoplasmosis is one of the most frequent infections in both animals and humans affecting approximately $30-50 \%$ of the total world human population ${ }^{[24,25]}$. Ophthalmoimmunologists suggested that even some six billion people are chronically infected with T. gondii, with high seroprevalence in tropical areas and low prevalence in cold regions $^{[26]}$. Seroprevalence increased markedly with age, to approximately $80-100 \%$ in persons aged $66-75$ years ${ }^{[26,27]}$. At present, however, in immunocompetent individuals $T$. gondii infection is still believed to be asymptomatic ${ }^{[28,29]}$, but a rapidly increasing body of literature strongly indicate that the parasite is slowly emerging as a global health threat ${ }^{24,25,30]}$.

Three clonal strain types (I, II, and III) of $T$. gondii predominate worldwide $^{[31]}$. In mice, the outcome of infection is highly dependent of the parasite genotype with type I strains being uniformly virulent, while types II and III are nonvirulent. Strains types II and III result in controlled infections that persist chronically ${ }^{[32]}$. The most prevalent in nature and frequent in humans infection with type II strain parasites stimulate NF- $\kappa \mathrm{B}$ nuclear translocation and lead to an abnormal overproduction of proinflammatory $\mathrm{T}_{\mathrm{H}} 1$ cytokines, including IFN- $\gamma$, TNF- $\alpha$, IL-1 $\beta$, IL-12 and IL-6, that are typically protective ${ }^{[31-34]}$. Different strains of $T$. gondii produce different cytokines and cause different clinical entities ${ }^{[30]}$, e.g. type I strain is more prevalent in ocular toxoplasmosis ${ }^{[30,35]}$.

The aim of the work was to review and critically analyze available literature data, especially focusing on the molecular pathomechanisms of both atherosclerosis and toxoplasmosis, and provide solid scientific documentation that latent chronic $T$. gondii infection probably play an essential role in triggering, development and progression of atherosclerosis in both animals and humans.

INCREASED SUSCEPTIBILITY OF VASCULAR
ENDOTHELIAL CELLS TO INFECTION WITH T.
gONDIi MAY BE A FREQUENT CAUSE OF THE
ENDOTHELIAL DYSFUNCTION IN PATIENTS
WITH ATHEROSCLEROSIS

Endothelial cells maintain the functional integrity of the vascular wall, and are involved in controling vascular wall permeability, the regulation of immune and inflammatory reactions, the modulation of blood flow and vascular resistance, and the maintenance of a nonthrombogenic blood-tissue interface ${ }^{[36]}$.

Damage of vascular endothelial cells by proatherosclerotic risk factors, such as proinflammatory cytokines, oxidized proteins/ lipids, and/or hyperglycemia, leads to endothelial dysfunction ${ }^{[37,38]}$. Accelerated apoptosis of vascular endothelium cells ${ }^{[39]}$ and an associated delay in cell cycle progression, affecting morphology and impairing function of the endothelial monolayer, are believed to be key bioevents for triggering and progression of micro- and 
macrovascular diseases, including premature atherosclerosis and diabetic neuropathy ${ }^{[37-39]}$

Endothelial cell dysfunction plays an important role in the process of atherosclerotic lesion formation by promoting leukocyte adhesion, increasing chemokine generation and cell permeability to lipids, enhancing LDL oxidation, stimulation of vascular smooth muscle cell proliferation and migration, and platelet activation ${ }^{[40]}$. Endothelial dysfunction is characterized by a reduction in the bioavailability of vasodilators, especially $\mathrm{NO}$, and the increase in production of endothelium-derived contractile factors. There is a state of endothelial cell activation associated with increased generation of proinflammatory, proliferative, and procoagulant biofactors, all favoring triggering and development of atherogenesis ${ }^{[4]}$. Endothelial dysfunction may be caused by acute and/or chronic inflammation state and enhanced production of proinflammatory cytokines and free radicals ROS and RNI. Increased LDL oxidation causes enhanced expression of adhesion molecules in the endothelium, which facilitates monocyte infiltration of subendothelial space ${ }^{[42]}$.

It must be noted that flow-mediated dilation (FMD) (the most widely used non-invasive ultrasound method to assess endothelial function) derived using a proximal or distal cuff may serve as future cardiovascular events prediction ${ }^{[42 a, 42 b]}$. Age- and sex-specific FMD data were early markers of atherosclerosis, and males had a lower FMD values than females, and FMD appeared to decline with age (except of pediatric population) ${ }^{[42 c-42 e]}$. Obstructive sleep apnea and hypertension also appeared to be early clinical markers of carotid atherosclerosis ${ }^{[42 f, 42 g]}$. In addition, plasma pentraxin 3 (PTX3) was found to be a more sensitive predictor of endothelial dysfunction than serum high-sensitive C-reactive protein (hSCRP) ${ }^{[2 h h]}$, at least in part because PTX3 overexpression in neutrophils was associated with intracellular ROS overproduction ${ }^{[42 i]}$.

\section{Vascular endothelial cells are susceptible to infection with $T$. gondii tachyzoites}

The parasite is disseminating in the body as a Trojan horse in various eukaryotic cells, including endothelial cells and macrophages, and division rate of intracellular unprimed T. gondii tachyzoites in endothelial cells, monocyte-derived macrophages, peritoneal, or alveolar cells is rapid (Table $\mathbf{1})^{[43]}$.

In congenital toxoplasmosis, the parasites first invaded the umbilical vein endothelial cells and were then disseminated throughout the fetus ${ }^{[60,61]}$. Canedo-Solares et al ${ }^{[60]}$ found that RH tachyzoites infected up to $19 \%$ human umbilical vein endothelial cells (HUVECs), while ME49 parasites invaded up to $63 \%$ human microvascular endotheial cell-1 line (HMEC-1). Both RH and Me49 tachyzoites invaded 46 and 49\% HUVECs and HMEC-1 cells, respectively. Reinvasion and formation of new parasitophorous vacuoles of infected cells was more frequent than invasion of noninfected cells. Thus, dissemination and vertical transmission of $T$. gondii appeared to be parasite strain type, host cell type/subtype and activation state- dependent. Replication speed of the parasite seemed to affect its virulence ${ }^{[60]}$.

Smith $e t a l^{[62]}$ found that retinal vascular endothelium cells have enhanced susceptibility to infection with $T$. gondii tachyzoites in comparison with aorta (55\% more), umbilical vein (33\%), and dermal endothelial cells ( $34 \%$ more), and tropism of different pathogens for particular cell types and/or specific tissue sites was a long-recognized biological phenomenon ${ }^{[62]}$. Free $T$. gondii tachyzoites had the ability to transmigrate a stimulated human retinal endothelium monolayer ${ }^{[63]}$. Tachyzoites crossed retinal endothelium assisted by intercellular adhesion molecule-1 (ICAM-1) (the cell surface IgG immunoglobin
Table 1 Division rate of intracellular $T$. gondii tachyzoites in primary human cells in vitro (acc. to Channon et $^{\left[l^{[3]}\right.}$; with own modification).

\begin{tabular}{|c|c|c|c|c|}
\hline Cell type & $\begin{array}{c}\text { Parasite } \\
\text { division } \\
\text { rate } \\
\text { Unprimed }\end{array}$ & $\begin{array}{c}\text { IFN- } \gamma \\
\text { primed }\end{array}$ & Mechanism & Refs \\
\hline \multicolumn{5}{|l|}{ Hemopoietic } \\
\hline Lymphocyte & $\mathrm{s}$ & & & [43] \\
\hline Neutrophil & $\mathrm{s}$ & & & [43-45] \\
\hline Adherent monocyte & $\mathrm{s}$ & & & [45-50] \\
\hline Nonadherent monocyte & $\mathrm{R}$ & $\mathrm{R}$ & ROS; not TS & {$[43,47]$} \\
\hline Dendritic cell & $\mathrm{R}$ & & & [43] \\
\hline Alveolar macrophage & $\mathrm{R}$ & $\mathrm{s}$ & Partly TS & [50] \\
\hline Peritoneal macrophage & $\mathrm{R}$ & $\mathrm{s}$ & & [51] \\
\hline $\begin{array}{l}\text { Monocyte-derived } \\
\text { macrophage }\end{array}$ & $\mathrm{R}$ & S & ROS; not RNI & [49-52] \\
\hline \multicolumn{5}{|l|}{ Nonhemopoietic } \\
\hline Neuron & S & & & [53] \\
\hline Foreskin fibroblast & $\mathrm{R}$ & $\mathrm{s}$ & TS & {$[54,55]$} \\
\hline $\begin{array}{l}\text { Umbilical vein endothelial } \\
\text { cell }\end{array}$ & $\mathrm{R}$ & S & TS or ROS & {$[49,56]$} \\
\hline Retinal pigment epithelial cell & $\mathrm{R}$ & $\mathrm{s}$ & TS & {$[57]$} \\
\hline Fetal astrocyte & $\mathrm{R}$ & $\mathrm{S}$ & RNI & {$[53,58]$} \\
\hline Fetal microglial cell & $\mathrm{R}$ & $\mathrm{R}$ & & [59] \\
\hline
\end{tabular}

$\mathrm{R}$, rapid; S, slow; RNI, reactive nitrogen intermediates; ROS, reactive oxygen species; TS, tryptophan starvation.

superfamily member) in vitro, and ICAM-1 blockade significantly (by approximately $50 \%, p<0.001$ ) inhibited the parasite migration across stimulated human retinal, but not choroidal, vascular endothelium ${ }^{[63]}$. There has been interest in the heterogeneity of vascular endothelium, not only between arteries and venous types ${ }^{[64]}$, but also between same type vessels located within different organs or within different tissues in the same organ ${ }^{[65,66]}$. An intracellular environment that is low in ROS or RNI and/or high in iron or tryptophan is known to facilitate tachyzoite proliferation ${ }^{[49,54,67]}$. Courret et $a{ }^{[68]}$ tracked fluorescently labeled tachyzoite-infected CD11c-positive or CD11b-positive leukocytes from blood to brain, and showed that human dendritic cells (DCs) and monocytes were more permissive to infection with $T$. gondii tachyzoites than neutrophils or lymphocytes ${ }^{[43]}$. Moreover, transmigration of human DCs across retinal endothelium was augmented following infection with the parasite ${ }^{[69]}$. In this context, it must be emphasized that nearly 1 million strokes occur in the US each year, and approximately 10$15 \%$ can be attributed to intracranial atherosclerotic disease ${ }^{[70]}$, and the infection of brain vascular endothelial cells with tachyzoites may play an important role in these clinical bioevents.

\section{Enhanced T. gondii adhesion to vascular endothelium during} disturbed blood flow

Atherosclerosis disease occurs at sites of disturbed blood flow, typically in the curvature of vessels or at branching points ${ }^{[71-76]}$. Smooth laminar blood flow induces NO production, which has multiple anti-atherosclerotic functions, including suppressing adhesion molecule expression by endothelial cells, inhibiting platelet aggregation, and inhibition proinflammatory cytokine production ${ }^{[77]}$. It was demonstrated that $T$. gondii modulated the dynamics of human monocyte adhesion to vascular endothelium under fluidic shear stress $^{[78]}$. T. gondii-infected primary human monocytes and THP-1 cells exhibited altered adhesion dynamics compared with uninfected monocytes. Infected cells rolled at markedly higher velocities (2.5- to 4.6-fold) and over greater distances (2.6- to 4.8-fold), than 
uninfected monocytes, before firmly adhering ${ }^{[78]}$. Moreover, T. gondii tachyzoites adhered and glided on human vascular endothelium under shear stress conditions ${ }^{[79]}$. Compared to static conditions, shear stress enhanced $T$. gondii helical gliding, resulting in a significantly greater displacement, and increased the percentage of tachyzoites that invaded and sustained adhesion to vascular endothelium. It appeared that the parasite surface protein MIC2 contributed to initial adhesion but was not required for adhesion strengthening ${ }^{[79]}$.

\section{Increasing T. gondii infection percentage during human aging}

Endothelial cells are a replicative niche for entry of the parasite to the brain vasculature, and replication in and lysis of these cells precedes invasion of the central nervous system ${ }^{[80]}$. Vascular endothelial dysfunction occurs during the human aging process and is accompanied by deterioration in the balance between vasodilator and vasoconstriction substances produced by the endothelium ${ }^{[81]}$. This imbalance is mainly characterized by a progressive reduction of the bioavailability of $\mathrm{NO}$ and an increase in the production of COX-derived vasoconstrictor factors. Both circumstances are in turn related to an increased generation of ROS and $\mathrm{RNI}^{[81]}$. Interestingly, the percentage of individuals chronically infected with $T$. gondii also is increasing with age (Table 2) and this may, at least in part, be responsible for development of vascular endothelial dysfunction during aging.

Proinflammatory and antiinflammatory cytokines acting as positive or inhibitory stimuli influence the development and maintenance of differentiated properties in the vasculature. In SMCs, the gene for the contractile protein, $\alpha$-actin, is stimulated by TGF- $\beta$ but inhibited by IFN- $\gamma^{[82,83]}$. Similarly, TGF- $\beta$ strongly promoted the synthesis of interstitial collagens types I and III by human SMCs, whereas IFN- $\gamma$ powerfully inhibited their synthesis of collagen, as well as $\alpha$-actin ${ }^{[84]}$. Vascular cells participate in and propagate the inflammatory response at sites of microbial challenge or pathological processes by generating large amounts of cytokines (Table 3). Both ECs and SMCs responded to stimulation with IL-1, TNF- $\alpha$, or CD40 ligand by producing great quantities of IL- $6^{[85]}$, and in one study of 14,916 apparently healthy men who developed myocardial infarction, plasma IL-6 levels were significantly elevated as compared with control individuals who did not have heart problems ${ }^{[86]}$.

\section{Impaired nitric oxide (NO) production in endothelial dysfunction} Vascular endothelium-derived NO, a major mediator of endotheliumdependent vasodilation, has important antiinflammatory and antithrombotic properties, such as inhibiting leukocyte adhesion, limiting platelet adhesion and aggregation, and the expression of PAI-1, a prothrombotic protein ${ }^{[88-90]}$. A common mechanism underlying endothelial dysfunction relates to inflammation and increased vascular production of ROS and RNI ${ }^{[00]}$. Endothelial cell tetrahydrobiopterin $\left(\mathrm{BH}_{4}\right)$, a naturally occurring essential cofactor for several critical metabolic pathways, including the production of $\mathrm{NO}$, is required for the maintenance of vascular function through enhanced $\mathrm{NO}$ production, and reduced levels of $\mathrm{BH}_{4}$ were associated with vascular dysfunction ${ }^{[76]}$. Stroes et al ${ }^{[88]}$ demonstrated that $\mathrm{BH}_{4}$ restored vascular endothelial function in patients with familial hypercholesterolemia which was associated with impaired NO activity. Recently, it was also however reported that although acute and short-term $\mathrm{BH}_{4}$ oral administration improved vascular endothelial function in patients with rheumatoid arthritis, the supplementation did not alter aortic stiffness (an independent risk factor for $\mathrm{CVD}^{[89]}$. The authors suggested that these two disturbances may occur in parallel and probably share common risk factor such as inflammation.
Table 2 Percentage of T. gondii positive individuals among 214 nonpsychiatrically affected controls depending on age analyzed during a large epidemiologic study of 869 psychiatric patients (Hinze-Selch et $\left.a l^{[27]}\right)$.

\begin{tabular}{|l|c|c|c|c|c|c|c|c|c|c|c|c|}
\hline $\begin{array}{l}\text { Percent } \\
\text { T. gondii } \\
\text { positive }\end{array}$ & $\begin{array}{c}18- \\
20\end{array}$ & $21-$ & $26-$ & $31-$ & $36-$ & $41-$ & $46-$ & $51-$ & $56-$ & $61-$ & $66-$ & $71-$ \\
\hline 100 & & & & & & & & & & & & 100 \\
\hline 80 & & & & & & & & & 80 & & 80 & \\
\hline 60 & & & & & & & 58 & 58 & & 60 & & \\
\hline 40 & & 35 & 32 & 40 & 39 & 38 & & & & & & \\
\hline 20 & 19 & & & & & & & & & & & \\
\hline 0 & & & & & & & & & & & & \\
\hline
\end{tabular}

In the control individuals 45 yrs or younger recruited from the same geographical region as the psychiatric patients admitted to the hospital, serofrequency of T. gondii infection ranged between 20 and $40 \%$ without any systematic age effect, whereas in the individuals older than $45 \mathrm{yrs}$ serofrequency systematically increased with age from about $40 \%$ to almost $100 \%^{[27]}$.

Table 3 Regulation of human vascular endothelial and smooth muscle cells function by positive and inhibitory stimuli (acc. to Hansson et al ${ }^{[82]}$; with own modification).

\begin{tabular}{|l|c|c|}
\hline Target & Positive stimuli & $\begin{array}{c}\text { Inhibitory } \\
\text { stimuli }\end{array}$ \\
\hline SMC proliferation & PDGF (TGF- $\beta$ ) & IFN- $\gamma$ (TGF- $\beta)$ \\
\hline SMC matrix/collagen & TGF- $\beta$ (IL- 1, PDGF) & IFN- $\gamma$ \\
\hline SMC contractile proteins & TGF- $\beta$ & IFN- $\gamma$ \\
\hline Ig-type adhesion molecules & IL-1, TNF- $\alpha$, IFN- $\gamma$, CD40L & \\
\hline SMC NOS2 & IL-1, TNF- $\alpha$, IFN- $\gamma$ & TGF- $\beta$, IL-4 \\
\hline EC/SMC COX-2 & IL- 1, TNF- $\alpha$, IFN- $\gamma$, CD $40 L$ & \\
\hline EC E-selectin & IL-1, TNF- $\alpha$, IFN- $\gamma$, CD40L & \\
\hline EC/SMC tissue factor & IL- 1, TNF- $\alpha$, CD40L & \\
\hline EC/SMC MMPs & IL- 1, TNF- $\alpha$, CD40L & IFN- $\gamma$ \\
\hline
\end{tabular}

EC, endothelial cell; Ig indicates immunoglobulin superfamily; CD40L, CD40 ligand (functional CD40L is expressed on human vascular ECs, SMCs, and macrophages; the activated T cells also express CD40L and in advanced human atherosclerotic plaques T cells constitute $10-20 \%$ of the cell population ${ }^{[53,55]}$ ); COX-2, cyclooxygenase 2; NOS2, nitric oxide synthase 2; MMPs, matrix metalloproteinases; PDGF, platelet-derived growth factor; SMC, smooth muscle cell.

Table 4 Intracellular pteridine levels in cultured and freshly isolated human umbilical vein endothelial cells (HUVEC) (acc. to RosenkranzWeiss et $a l^{[91]}$;with own modification).

\begin{tabular}{|c|c|c|}
\hline \multirow{3}{*}{ HUVEC conditions } & \multicolumn{2}{|c|}{ Pteridine levels } \\
\hline & Biopterin & Neopterin \\
\hline & \multicolumn{2}{|c|}{ pM per million cells } \\
\hline \multicolumn{3}{|l|}{ Cultured, passage 3-6 } \\
\hline Control (4) & $<2$ & $<2$ \\
\hline TNF, IL-1 $\beta$, and IFN- $\gamma(4)$ & $27.6 \pm 6.7^{\mathrm{a}}$ & $24.6 \pm 11^{\mathrm{a}}$ \\
\hline Cultured, primary (3) & $<2$ & $<2$ \\
\hline Freshly isolated (3) & $31.7 \pm 1.3^{a}$ & ND \\
\hline
\end{tabular}

Results are mean \pm SEM. ${ }^{\text {a }} p<0.05$ vs control cultured HUVEC. Numbers in parentheses represent the number of samples. ND, not done.

Rosenkranz-Weiss et al ${ }^{[91]}$ demonstrated that proinflammatory cytokines such as IFN- $\gamma$ plus TNF or IL- $1 \beta$ enhanced NOS specific activity in cultured human endothelial cells by increasing intracellular BH4 levels (Table 4).

\section{Effect of other putative vascular cell pathogens on endothelial function}

The first line of immune host defense is based on detection of pathogen-associated molecular patterns that evoke inflammatory and 
toxic response ${ }^{[92,93]}$. Several studies suggested a role for Chlamydia pneumoniae (CP) in atherosclerosis ${ }^{[21]}$. For example, HSP60 of this microbe resembles human HSP60 and can elicit inflammatory response ${ }^{[94]}$. Chlamydial and human HSP60 induced TNF- $\alpha$ and MMP production by macrophages ${ }^{[95]}$ and stimulated E-selectin, ICAM-1, and VCAM-1 expression on endothelial cells ${ }^{[94]}$. HSP60 also markedly enhanced IL-6 production by endothelial and smooth muscle cells, and macrophages ${ }^{[94]}$. Another putative vascular pathogen, cytomegalovirus (CMV), encoded a chemokine receptor that rendered infected smooth muscle cells susceptible to CC chemokine-induced migration ${ }^{[96]}$.

Several authors demonstrated the relationship between the seropositivity to CP, HSV-1 and 2, HCMV, HAV, HP, EBV, and atherosclerosis/coronary artery disease ${ }^{[97-101]}$. Recently, it was also suggested that more infectious agents rather than any single pathogen may be involved in the development of atherosclerosis and the subsequent cardiovascular events because the proatherogenic effect of each infectious organism might be amplified ${ }^{[1,102]}$. Specifically, in 86 patients with no angiographically significant CAD, Prasad et $a l^{[103]}$ found a significant correlation between total infection burden to five investigated agents ( $\mathrm{CP}, \mathrm{CMV}, \mathrm{H}$. pylori, hepatitis A, and herpes simplex virus) and decreased intracoronary endotheliumdependent vasodilation $(p=0.03)$. [Of note, the eventual presence of latent chronic T. gondii infection in these patients had not been investigated]. The inflammatory state can induce oxidative stress by enhancing the production of ROS in the vascular wall, therefore contributing to the progression and destabilization of atherosclerotic plaque and consequently to $\mathrm{CADs}^{[104]}$. Key process in the development of atherosclerosis is LDL oxidation and accumulation in vascular cells, promoting foam cell formation, as well as increased secretion of mediators of inflammation, including IL-1, IL-6 and TNF- $\alpha^{[104]}$

On the other hand, Khairy et $a l^{[105]}$ found lack of association between chronic infection with $\mathrm{CP}, \mathrm{CMV}, \mathrm{EBV}, \mathrm{HP}$, or pathogen burden, and endothelial function in 65 male individuals, aged 20 to $45 \mathrm{yrs}$ (mean age $=29.3 \pm 5.5 \mathrm{yrs}$ ), with no risk or known coronary artery disease(CAD), suggesting that these agents were not implicated as early etiologic triggers in the pathophysiology of CAD (Table 5). Endothelial function was determined by flowmediated brachial artery vasodilation and seroprevalence values were $65.1 \%, 34.9 \%, 88.9 \%$, and $14.3 \%$, for $\mathrm{CP}, \mathrm{CMV}, \mathrm{EBV}$, and $\mathrm{HP}$, respectively ${ }^{[105]}$, consistent with the earlier values reported in young patient populations ${ }^{[106,107]}$, and the coexistence of these infections has been well described ${ }^{[108]}$.

In summary, latent chronic $T$. gondii infection may be a frequent cause of vascular endothelial cell dysfunction because the parasite attacks all eukaryotic cells and endothelium cells have enhanced susceptibility to infection with $T$. gondii tachyzoites with rapid division rate. Increase of vascular endothelial dysfunction occurs during the human aging process and it was found that the percentage of individuals chronically infected with $T$. gondii also is increasing with age. Human studies showed that the infection burden such as H. pylori, $\mathrm{CMV}$, and $\mathrm{EBV}$, decreased endothelium-dependent vasodilation, but eventual additional latent chronic $T$. gondii infection in these participants had not been investigated.

\section{OXIDATIVE STRESS CHARACTERISTIC FOR ATHEROSCLEROSIS MAY BE CAUSED BY LATENT CHRONIC T. gondii INFECTION}

Chronic inflammation (innate immunity-associated) may trigger
Table 5 Flow-mediated brachial artery vasodilation caused by chronic low-grade infection with Chlamydia pneumoniae, Ebstein-Barr virus, cytomegalovirus, and H. pylori, associated with decreased endothelial function in seropositive healthy young men (acc. to Khairy et al ${ }^{[105]}$; with own modification).

\begin{tabular}{|l|c|c|c|c|}
\hline Infectious agent & $\begin{array}{c}\text { FMD } \\
\text { Seropositive }\end{array}$ & $\begin{array}{c}\text { FMD } \\
\text { Seronegative }\end{array}$ & $\begin{array}{c}\text { Parameter } \\
\text { estimate } \\
\mathbf{9 5 \%} \text { CI }\end{array}$ & $P$ \\
\hline $\begin{array}{l}\text { Chlamydia } \\
\text { pneumoniae }\end{array}$ & $9.8 \pm 4.5$ & $8.5 \pm 4.5$ & $0.280(-2.053,2.613)$ & 0.8151 \\
\hline $\begin{array}{l}\text { Ebstein-Barr } \\
\text { virus }\end{array}$ & $9.5 \pm 4.5$ & $8.1 \pm 4.2$ & $0.159(-0.432,0.750)$ & 0.6006 \\
\hline Cytomegalovirus & $9.0 \pm 4.5$ & $9.5 \pm 4.5$ & $-0.806(-3.403,1.789)$ & 0.5452 \\
\hline H. pylori & $13.6 \pm 4.4$ & $8.7 \pm 4.1$ & $2.888(-0.354,6.129)$ & 0.0888 \\
\hline
\end{tabular}

FMD, flow-mediated brachial dilation; CI, confidence interval.

initial events that can lead to atherosclerotic cardiovascular disease ${ }^{[109]}$. Early stages of chronic inflammation and onset of atherosclerosis may be evident in embryonic life ${ }^{[110]}$, and be perpetuated by classic atherosclerosis environmental risk factors, including $T$. gondii infection. Lipid peroxidation and formation of oxLDL are pivotal processes in the development of atherosclerosis that represent a crucial proinflammatory stimulus ${ }^{[11,112]}$. Atherosclerosis and autoimmune diseases have a number of pathogenic similarities ${ }^{[113]}$, and autoimmunity plays an essential role in pathophysiology of atherogenesis. Atherosclerosis triggers the production of autoantibodies against substances such oxLDL ${ }^{[113]}$.

Oxidation of LDL result in the formation of reactive aldehyde products such as malondialdehyde (MDA), 4-hydroxynonenal (4HNE), or 1-palmitoyl-2-oxovaleroyl phosphatidylcholine ${ }^{[14]}$. MDAmodified LDL is commonly utilized as an indicator for oxLDL, because MDA is the most abundant aldehyde arising from lipid peroxidation. Recently, Matsuo et a ${ }^{[115]}$ demonstrated that circulating MDA-LDL concentrations were associated with the presence of thin-cap fibroatheromas in patients with coronary artery disease ${ }^{[115]}$. Bardag-Gorce et al ${ }^{[116]}$ showed that 4-HNE inhibited the proteasome activity and that the proteasome was unable to degrade proteins heavily modified by 4-HNE. Ultrastructurally, 4-HNE-treated cells displayed extensive vacuole formation, pinocytic body formation, crescent-shaped phagophores, and multilamellar vesicles ${ }^{[117]}$. In addition, 7-ketocholesterol present in oxLDL, not only triggers oxidative damage and protein-4-HNE modification but also extensive vacuolization in $\mathrm{SMCs}^{[118]}$. OxLDL is also a potent chemoattractant and induces the secretion of macrophage-chemotactic protein- 1 by endothelial cells ${ }^{[119-121]}$.

Acute or persistent oxidative stress results in an intracellular increase of ROS that damage the lysosomal membrane. When oxidative injury overcomes the cellular defenses, cells probably die via apoptosis ${ }^{[117,122]}$. The production of ROS and RNI (peroxynitrite, i.e. $\mathrm{ONOO}^{-}$, a potent oxidant and nitrating agent that can cause formation of several lipid oxidation products including MDA, lipid peroxides and lipid hydroxides, and oxysterols, as well as interaction iron and copper species ${ }^{[123-125]}$ has been implicated in atherosclerosis principally as means of damaging $\operatorname{LDL}^{[126-128]}$. Oxidation of lipids present in LDL generate a broad range of reactive products such as the above-mentioned aldehydes that are capable of reacting with lysine residues ${ }^{[123]}$. Specifically, the extent of oxidation of linoleic acid measured in atherosclerotic lesion is approximately $20 \%$ and is similar to in vitro studies using copper-mediated LDL oxidation ${ }^{[29]}$. Antibodies directed to specific oxidized lipid-protein adducts cross react with material in atherosclerosis, and titers of autoantibodies to oxLDL are positively correlated with the progression of atherosclerotic lesions in humans ${ }^{[130]}$. 


\section{Latent chronic $T$. gondii infection}

Several studies suggested that infectious pathogens, including T. gondii, H. pylori, Chlamydia peumoniae, and Enterococcus fecalis $^{[131-133]}$ may be responsible for inducing oxidative stress and triggering primary atherosclerotic lesions in both animals and humans ${ }^{[131,134,135]}$. T. gondii infection may be at least partly responsible for development of these abnormalities because Yang et $a l^{[136]}$ showed that in mice injected intraperitoneally with 2.5 $\times 10^{3} T$. gondii tachyzoites, the serum level of the oxygen free radicals $\left(\mathrm{NO}^{+}, * \mathrm{OH}, \mathrm{O}_{2}^{-}\right)$increased along with the days of infection, and superoxide dismutase concentration reached a peak on the $3^{\text {rd }}$ day of the infection. It was documented that oxidative stress with markedly increased serum MDA and decreased glutathione levels is characteristic for chickens orally infected with tissue cysts of the protozoan $^{[133]}$ (Tables 6 and 7), and T. gondii seropositive patients (Table 8) ${ }^{[137,138]}$.

Al-Kennany ${ }^{[133]}$ demonstrated a lipid peroxidation status in the aortic and heart tissue of T. gondii-infected broiler chickens with a significant increase in MDA level and a marked decrease in glutathione concentrations in these tissues. Histopathological examination revealed that aortic lesions had thickening of the wall due to accumulation of lipid vacuoles in tunica media extending towards tunica intima, associated with proliferation and hypertrophy of VSMCs, hypertrophy of endothelial cells with invasion of tachyzoites in these cells ${ }^{[133]}$. Some aortic sections revealed accumulation of tachyzoites in the cytoplasm of VSMCs, well as an accumulation of lipid vacuole concomitant with foamy cells in both tunica media and intima with fragmentation of elastic lamellae and infiltration of lymphocytes ${ }^{[133]}$ (Table 6).

In asymptomatic $T$. gondii-seropositive blood donors, oxidative stress and immunosuppression have been demonstrated ${ }^{[139,140]}$. Foroutan-Rad et al ${ }^{[141]}$ searched in 7 electronic databases information on frequency of toxoplasmosis in blood donors, and in total 43 records which included 20,964 donors from 1980 to 2015, they found that the overall weighted prevalence of exposure was $33 \%$ (95\% CI, 28-39\%). The highest and the lowest seroprevalences of toxoplasmosis were observed in Africa (46\%; $95 \mathrm{CI}, 14-78 \%$ ) and in Asia (29\%; 95\% CI, 23-35\%), respectively. Brazil and Ethiopia were identified as countries with high anti-T. gondii seroprevalence ${ }^{[141]}$.

Elsheikha et a ${ }^{[139]}$ found in a group of 260 blood donors that the plasma concentration of MDA was significantly higher and activity of glutathione peroxidase and level of tocopherol ( $\alpha, \gamma$ and $\sigma$ ) fractions $(p<0.001)$ were lower in T. gondii- IgG seropositive than

Table 6 Levels of malondialdehyde (MDA) and glutathione (GSH) in aorta and heart of broiler chickens infected intraperitoneally with 50 tissue cysts of T. gondii (acc. to Al-Kennany ${ }^{[133]}$; with own modification).

\begin{tabular}{|l|c|c|c|c|}
\hline Groups & $\begin{array}{c}\text { MDA } \\
\text { (nM per gm } \\
\text { wet tissue) } \\
\text { Aorta }\end{array}$ & Heart & $\begin{array}{c}\text { GSH } \\
\text { (mM per gm } \\
\text { wet tissue) } \\
\text { Aorta }\end{array}$ & Heart \\
\hline Controls & $125 \pm 1.05$ & $105 \pm 0.33$ & $0.622 \pm 0.022^{\mathrm{a}}$ & $0.773 \pm 0.026$ \\
\hline Infected & $529.8 \pm 2.32^{\mathrm{a}}$ & $455.3 \pm 0.43^{\mathrm{a}}$ & $0.273 \pm 0.01$ & $0.421 \pm 0.031$ \\
\hline
\end{tabular}

Results are expressed as means \pm SD of 10 broiler chickens per group.

${ }^{\text {a }}$ Significant differences at $p \leq 0.05$. in seronegative blood donors. The authors suggested that there was a degradation of their antioxidant enzymes caused by oxidative stress induced by increased free radicals attributed to $T$. gondii infection. It must be noted that glutathione induces egress of the parasite from infected cells by activating a $T$. gondii-secreted apyrase (nucleoside triphosphate hydrolase) in the parasite vacuole resulting in a rapid depletion of host cell $\mathrm{ATP}^{[142]}$.

Interestingly, Jafari et $a l^{[143]}$ in the case-control study demonstrated that women were more sensitive to the effects of $T$. gondii infection on oxidative stress induction compared to men. Overall, 75 (50\%) of 150 participants 20-50 yrs old were seropositive for anti-T. gondii IgG antibodies, including 23 (15.33\%) men and 52 (34.66\%) women; the control group of participants consisted of 75 individuals (32 men and 43 women) with serum anti-T. gondii $\operatorname{IgG}$ negative samples. Serum catalase activity (converts $\mathrm{H}_{2} \mathrm{O}_{2}$ to $\mathrm{H}_{2} 0$ ) was significanlty increased in men of the case group $(p=0.006)$, while it was decreased in women of this group, as compared with controls ( $p=$ 0.043 ). Catalase activity in women of the case group was markedly lower than that of men $(p=0.003)^{[143]}$. Effects of $T$. gondii infection on serum glutathione and MDA levels in the case and control groups were presented in Table 9.

Vegeto et $a l^{[144]}$ showed that estrogens may block iNOS activity and accumulation in LPS-activated microglia cells, as well as reduce the accumulation of nitrites and nitrates consequent to various inflammatory stimuli, and thus play a protective role against neurodegeneration.

Oxidative stress plays an important role also in the pathogenesis of neurodegenerative diseases. Dincel and Atmaca ${ }^{[145]}$ demonstrated in the brain tissue of mice infected with ME49 strain T. gondii that

Table 8 Serum glutathione, malondialdehyde, and NO concentrations in T. gondii-seropositive patients and healthy controls (acc. to Karaman et $a l^{[137]}$; with own modification.

\begin{tabular}{|l|l|l|l|l|}
\hline \multirow{2}{*}{ Bioparameter } & Group & $\begin{array}{l}\text { No of } \\
\text { participants }\end{array}$ & $\begin{array}{l}\text { Serum levels } \\
\text { (mean } \pm \text { SD) }\end{array}$ & $\boldsymbol{P}$ values \\
\hline \multirow{2}{*}{ Glutathione } & Patients & 37 & $3.96 \pm 0.10$ & 0.001 \\
\cline { 2 - 5 } & Controls & 40 & $10.37 \pm 0.13$ & \\
\hline \multirow{2}{*}{ Malondialdehyde } & Patients & 37 & $41.32 \pm 2.05$ & 0.001 \\
\cline { 2 - 5 } & Controls & 40 & $9.18 \pm 1.21$ & \\
\hline \multirow{2}{*}{ NO } & Patients & 37 & $47.47 \pm 1.00$ & 0.001 \\
\cline { 2 - 5 } & Controls & 40 & $39.18 \pm 1.29$ & \\
\hline
\end{tabular}

Results statistically significant at $p<0.05$. Serum glutathione and NO levels are expressed as $\mu \mathrm{M}$, and malondialdehyde concentrations represent $\mathrm{nM}$.

Table 9 Effects of T. gondii infection on serum glutathione (GSH; an antioxidant) and malondialdehyde (MDA - a lipid peroxidation marker) levels in anti-toxoplasma IgG-seropositive participants and IgGseronegative controls (acc. to Jaffari et $\mathrm{al}^{[143]}$; with own modification).

\begin{tabular}{|l|c|c|c|c|}
\hline Parameters & \multicolumn{2}{|c|}{ Controls } & $\begin{array}{c}\text { T. gondii IgG- seropositive } \\
\text { participants }\end{array}$ \\
\hline & $\begin{array}{c}\text { Men } \\
(n=43)\end{array}$ & $\begin{array}{c}\text { Women } \\
(n=32)\end{array}$ & $\begin{array}{c}\text { Men } \\
(n=23)\end{array}$ & $\begin{array}{c}\text { Women } \\
(n=52)\end{array}$ \\
\hline Weight (kg) & $36.744 \pm 1.185$ & $34.437 \pm 1.382$ & $39.565 \pm 1.415$ & $36.077 \pm 0.779$ \\
\hline GSH (nM protein) & $0.838 \pm 0.059$ & $0.891 \pm 0.046$ & $0.757 \pm 0.075$ & $0.622 \pm 0.053^{\mathrm{a}}$ \\
\hline MDA (nM protein) & $1.669 \pm 0.099$ & $1.902 \pm 0.086$ & $1.832 \pm 0.107$ & $2.305 \pm 0.095^{\mathrm{b}, \mathrm{c}}$ \\
\hline
\end{tabular}

Results are mean \pm SE. ${ }^{\mathrm{a}} p=0.032$ and ${ }^{\mathrm{b}} p=0.007$ vs the controls; ${ }^{\mathrm{c}} p=0.028$ vs infected men.

Table 7 Plasma lipid profile of broiler chickens infected intraperitoneally with 50 tissue cysts of T. gondii (acc. to Al-Kennany ${ }^{[25]}$ with own modification).

\begin{tabular}{|l|l|l|l|l|l|l|}
\hline Groups & Total cholesterol mg/dL & Triglycerides mg/dL & HDL-C mg/dL & LDL-C mg/dL & VLDL-C mg/dL & Atherogenic index \\
\hline Controls & $117 \pm 0.23$ & $105.3 \pm 1.28$ & $42.06 \pm 2.72^{\text {a }}$ & $53.88 \pm 1.25$ & $21.06 \pm 0.02$ \\
\hline Infected & $478 \pm 0.30^{\text {a }}$ & $192.3 \pm 0.48^{\text {a }}$ & $33.36 \pm 1.04$ & $406.81 \pm 1.04^{\text {a }}$ & $38.49 \pm 1.28^{\text {a }}$ & $14.17 \pm 0.81^{\text {a }}$ \\
\hline
\end{tabular}

Results are expressed as means \pm SD of 10 broiler chickens per group. ${ }^{a}$ Significant differences at $p \leq 0.05$. HDL-C, high density lipoprotein-cholesterol; LDL-C, low density lipoprotein-cholesterol; VLDL-C, very low density lipoprotein-cholesterol. 
the levels of glutathione reductase $(p<0.005)$ and neuron specific enolase $(p<0.001)$ expression markedly increased, while $\mathrm{Cu} / \mathrm{Zn}$ superoxide dismutase activity decreased $(p<0.001)$ compared to the noninfected animals. Moreover, intense staining for 8-hydroxy-2'deoxyguanosine (a critical biomarker of oxidative stress) $(p<0.05)$ was observed both in the nucleus and the cytoplasm of neurons and glial cells that underwent oxidative stress ${ }^{[145]}$.

Together, there is the vicious circle between oxidative stress and inflammation in atherosclerosis ${ }^{[104]}$. Latent chronic T. gondii infection play an important role in inducing local/systemic inflammation and oxidative stress in both animals and humans. Since the parasite attacks almost all nucleated cells in mammals and slowly is becoming a global hazard ${ }^{[30]}$, it seems that it have a pivotal role in development of atherosclerosis.

\section{IMPORTANT ROLE OF T. gondii INFECTION IN THE PATHOGENESIS OF ATHEROSCLE- ROSIS}

The parasite has the autonomous capacity to synthesize phospholipids, and also readily scavenges precursors of the lipids from the host cell ${ }^{[146-148]}$. T. gondii is unable to synthesize sterol and acquires cholesterol from the host LDL receptor (LDLR) pathway ${ }^{[149]}$. LDLs are internalized by the LDLR or scavenger receptors and delivered to the late endosomes/lysosomes for hydrolysis ${ }^{[148]}$. Deposition of excess cellular cholesterol in the form of CEs is

Table 10 Uptake of cholesterol by mouse embryonic fibroblasts (MEF) $\mathrm{ACAT}^{-1}$ cells transfected with TgACAT1 or TgACAT2, and incorporation into cholesteryl esters (acc. to Lige et al ${ }^{[153]}$; with own modification).

\begin{tabular}{|c|c|c|}
\hline $\begin{array}{l}\text { MEF ACAT }^{--} \\
\text {transfected with }\end{array}$ & $\begin{array}{l}\text { Cholesterol uptake } \\
\left(\mathrm{cpm} \times \mu \mathrm{g} \text { cell protein }{ }^{-1}\right)\end{array}$ & $\begin{array}{l}\text { Cholesterol ester levels } \\
\left(\mathrm{cpm} \times \mu \mathrm{g} \text { cell protein }{ }^{-1}\right)\end{array}$ \\
\hline Vector & $3991 \pm 601$ & ND \\
\hline TgACAT1 & $4003 \pm 510$ & $898 \pm 65^{\mathrm{a}}$ \\
\hline TgACAT2 & $3758 \pm 436$ & $1514 \pm 110^{b}$ \\
\hline
\end{tabular}

The results are mean values \pm SD from three incubations (" $a$ " $v S$ " $b$ " is statistically significant, $p<0.01)$. MEF $\mathrm{ACAT}^{-1}$ were transfected with vector alone, or plasmids containing either TgACAT1 or TgACAT2. Cells were then incubated with $\left[{ }^{3} \mathrm{H}\right]$ cholesterol-LDL. Cholesterol uptake was monitored by scintillation counting. Cholesterol ester detection was performed by thin-layer chromatography analysis. Cpm, counts per minute; ND, not detected.

Table 11 Comparison of free fatty acids (FFAs) uptake by T. gondii and $\mathrm{CHO}$ cells (acc. to Quittnat et al ${ }^{[156]}$; with own modification).

\begin{tabular}{|l|c|c|c|r|c|}
\hline & Oleate & Palmitate & Stearate & Linoleate & Arachidonate \\
\hline T. gondii & $780 \pm 99$ & $1833 \pm 105^{\text {a }}$ & $810 \pm 69$ & $750 \pm 91$ & $767 \pm 77$ \\
\hline CHO cells & $1230 \pm 142$ & $1156 \pm 101$ & $1467 \pm 151$ & $1350 \pm 98$ & $1058 \pm 103$ \\
\hline
\end{tabular}

Results are means \pm SD of four separate experiments. Differences between values of the uptake of palmitate $v$ s other FFAs in T. gondii are statistically significant. ${ }^{a} p<0.005$. CHO, Chinese hamster ovary. Extracellular parasites or $\mathrm{CHO}$ cells were incubated for $1 \mathrm{~h}$ at $37^{\circ} \mathrm{C}$ with the indicated $0.3 \mathrm{mM}$ radioactive FFAs. After washing, the total FFAs uptake was determined by measuring the cell-associated radioactivity and expressed in $\mathrm{nM}$ per $\mathrm{mg}$ cell protein $/ \mathrm{h}$. catalyzed by the resident ACAT, leading to production of lipid bodies $^{[150,151]}$.

Sakashita et a ${ }^{[152]}$ demonstrated that immature human macrophages expressed only ACAT1, but the fully differentiated macrophages expressed both ACAT1 and ACAT2, and revealed the presence of these two enzymes mRNAs in human atherosclerotic aorta. Immunohistochemical study indicated that in the atherosclerotic aorta all macrophages expressed ACAT1, while approximately $70-80 \%$ of the cells also expressed ACAT2. It was suggested that in atherosclerotic plaque, the ability of macrophage foam cell transformation may be enhanced by the dual expression of ACAT1 and ACAT2 ${ }^{[152]}$. It must be emphasized that the marked expression of these two enzymes present in macrophages may be further augmented by the superimposing $\operatorname{TgACAT} 1$ and $\operatorname{TgACAT} 2$ activities available in the cells infected with $T$. gondii (Table 10) ${ }^{[153]}$.

T. gondii expresses two CE-synthesizing enzymes TgACAT1 and $T g A C A T 2$ that localize to the parasite ER, and TgACAT2 shares $56 \%$ identity with $\operatorname{TgACAT} 1^{[153,154]}$. Both these enzymes contribute to the CEs formation for storage in lipid bodies, and while $T g$ ACAT1 preferentially utilizes palmitoyl-CoA, $T g A C A T 2$ produces more CEs because it has a broader fatty acid specificity ${ }^{[154-156]}$. The parasite was competent to synthesize CEs using host cell-derived cholesterol and fatty acid, and the production was proportional to the amount of lipid taken up by the protozoan over the time ${ }^{[154,157]}$. It was demonstrated in vitro that perfusion of LDL in the culture medium lead to massive uptake of cholesterol by the parasite, suggestive of uncontrolled uptake of this lipid by $T$. gondii ${ }^{[153]}$. It should be emphasized that ACAT and CEs play a crucial role in replication of the pathogen ${ }^{[157]}$.

It appeared that several FFAs were incorporated into parasite $\mathrm{CE}$ but nearly twofold higher incorporation of palmitate into CE as compared with oleate, arachidonate, stearate, and linoleate was observed $^{[154,156]}$ (Tables 11 and 12). The preferential palmitate incorporation specificity by $T$. gondii acyl-CoA: diacylglycerol acyltransferase (TgDGAT1) has already been found also for triacylglycerol synthesis in the parasite ${ }^{[158]}$ (triacylglycerols are quantitatively the most important storage form of energy for eukaryotic cells), and TgDGAT1 is an integral membrane protein localized to the parasite cortical and perinuclear endoplasmic reticulum ${ }^{[156]}$. The protozoan was able to take up both oleate and diacylglycerol and incorporate them into the triacylglycerol fraction, and oleate was incorporated into diacylglycerol (Table 13).

Table 13 Lipid acquisition and substrate incorporation into n-acylglycerides of $T$. gondii (acc. to Quittnat et al ${ }^{[156]}$; with own modification).

\begin{tabular}{|l|c|c|c|}
\hline & $\begin{array}{c}\text { Lipid uptake }(\mathrm{cpm} \\
\text { per } \mu \mathrm{g} \text { cell protein) }\end{array}$ & $\begin{array}{c}\text { Lipid synthesis }(\mathrm{cpm} \\
\text { per } \mu \mathrm{g} \text { cell protein) DAG }\end{array}$ & TAG \\
\hline$\left[{ }^{3} \mathrm{H}\right]$ Oleate & $78589 \pm 190$ & $10444 \pm 1874$ & $32400 \pm 3880$ \\
\hline$\left[{ }^{14} \mathrm{C}\right] \mathrm{DAG}$ & $58430 \pm 3209$ & $39650 \pm 4705$ & $23664 \pm 2099$ \\
\hline
\end{tabular}

The values are means \pm SD of three separate experiments. DAG, diacylglycerol; TAG, triacylglycerol; cpm, counts per minute. Extracellular T. gondii were incubated for $1 \mathrm{~h}$ at $37^{\circ} \mathrm{C}$ with radioactive oleate or DAG before measurement of total lipid associated to parasites and lipid incorporation into DAG or TAG.

Table 12 Fatty acid specificity for triacylglyceride (TAG) synthesis in T. gondii (acc. to Quittnat $e^{\text {a }} l^{[156]}$; with own modification).

\begin{tabular}{|l|c|c|c|c|c|}
\hline & $\begin{array}{c}\text { Relative activities } \\
{\left[{ }^{3} \mathrm{H}\right] \text { oleate + oleate }}\end{array}$ & {$\left[{ }^{3} \mathrm{H}\right]$ oleate + palmitate } & {$\left[{ }^{3} \mathbf{H}\right]$ oleate + stearate } & {$\left[{ }^{3} \mathbf{H}\right]$ oleate + linoleate } & {$\left[{ }^{3} \mathbf{H}\right]$ oleate + arachidonate } \\
\hline T. gondii & 1.00 & 0.18 & 0.69 & 0.50 \\
\hline CHO cells & 1.00 & 0.52 & 0.62 & 0.61 \\
\hline
\end{tabular}

The values expressed as relative activities, are means of three separate experiments. CHO, Chinese hamster ovary. Extracellular T. gondii and CHO cells were incubated for $1 \mathrm{~h}$ at $37^{\circ} \mathrm{C}$ with $10 \mathrm{nM}$ of radioactive oleate $(\sim 50,000 \mathrm{dpm}$ per nM) previously mixed with $0.3 \mathrm{mM}$ of the indicated unlabeled FFAs to measure its incorporation into TAG. The production of TAG with oleic acid alone was $45,950 \mathrm{cpm}$ per mg cell protein for T. gondii. 
In atherosclerotic patients as well as during chronic latent $T$. gondii infection there is an increased production of proinflammatory cytokines that enhances both ACAT1 and ACAT2 activity in the infected host liver hepatocytes, intestine cells, and macrophages ${ }^{[159-163]}$. The enhanced activity of these enzymes will thus be overlapping with $\operatorname{TgACAT} 1$ and $\operatorname{TgACAT} 2$ activities in the intracellular tachyzoites. As a result, there will be an accumulation of a marked surplus of these enzymes activity finally leading to a more efficacious generation of foam cells, and development of several liver and/or intestine abnormalities, such as steatohepatitis or clear cell colitis ${ }^{[164,165]}$. These cells morphologically represent cholesterol ester-laden macrophages/foam cells, because the number and the size of lipid bodies in $T$. gondii vary between the different parasite developmental stages (tachyzoites, bradyzoites, oocysts), environmental conditions ${ }^{[153,166]}$, infected cells/tissues of the host, and intensity of oxidative stress associated with the infection.

Atherosclerosis was found to be more common in children with fatty liver $(30 \%)$ than in those without this abnormality $(19 \%)^{[167,168]}$. Nonalcoholic fatty liver disease (NAFLD) is the most common of chronic liver disease in Western countries that affects about 20$30 \%$ of the general population and is closely related to oxidative stress and insulin resistance ${ }^{[164,169-171]}$. The prevalence of NAFLD was about $3-10 \%$ in lean children, and about $53 \%$ in obese pediatric population ${ }^{[172]}$. Its histologically proven prevalence in children in the US (as found at autopsy after accidents) ranges from $9.6 \%$ in normalweight individuals up to $38 \%$ in obese ones ${ }^{[173]}$. This clinical entity includes a wide spectrum of liver diseases ranging from steatosis alone, to nonalcoholic steatohepatitis, which may progress to liver fibrosis and cirrhosis ${ }^{[170]}$. NAFLD is associated with coronary artery disease and subclinical atherosclerosis, emphasizing the role of chronic inflammation in the pathogenesis of atherosclerosis ${ }^{[171]}$. Autopsy findings in 817 children who died of external causes showed that fatty liver was present in $15 \%$ of the children, mild atherosclerosis in $21 \%$, and moderate to severe atherosclerosis was observed in $2 \%{ }^{[172]}$. These findings emphasize the importance of latent chronic $T$. gondii infection as a potential but so far neglected cause of atherosclerosis sometimes associated with unexplained liver damage and other clinical entities involving this organ, because this widely disseminated pathogen slowly becomes a global health hazard $^{[30,164]}$.

In summary, it was demonstrated that ACAT and CEs play a crucial role in replication of $T$. gondii. Immature human macrophages expressed only ACAT1, but the fully differentiated macrophages expressed both ACAT1 and ACAT2, and revealed the presence of these two enzymes mRNAs in human atherosclerotic aorta. Moreover, immunohistochemical study indicated that in that aorta, all macrophages expressed ACAT1, while approximately $70-80 \%$ of macrophages also expressed ACAT2. In congenital hyperlipidemic mice, ACAT2 was also present in lipid-laden cells of the atherosclerotic plaques. It was suggested that in atherosclerotic plaque, the ability of macrophage foam cell transformation may be augmented by the dual expression of ACAT1 and ACAT2. The marked expression of these two enzymes in macrophages may be further amplified by the $T g A C A T 1$ and $T g A C A T 2$ of $T$. gondii present in the infected host macrophages, finally leading to a more efficacious and faster building-up of atherosclerotic plaques.

\section{ACAT1 AND ACAT2 PLAY A CRUCIAL ROLE IN FOAM CELL FORMATION AND DEVEL- OPMENT OF ATHEROSCLEROTIC PLAQUE.}

UNCONTROLLED UPTAKE OF CHOLESTEROL AND SYNTHESIS OF LIPIDS BY T. gondii IS DUE TO THE INTRACELLULAR REPLICATION OF THE PARASITE

\section{Atherosclerosis}

It is accepted that atherosclerosis involves chronic inflammatory reaction ${ }^{[175]}$. The accumulation of foamy transformed macrophages in the aortic intima is a pathological hallmark of the early phase of atherosclerosis ${ }^{[176,177]}$. The initial process of foam cell formation is migration of blood monocytes into the vascular wall followed by their differentiation into macrophages, and their conversion into foam cells, which are characterized by intracellular accumulation of cholesteryl esters $(\mathrm{CEs})^{[178]}$. In the later stages of atherosclerosis, foam cell undergo apoptosis and secondary necrosis, which causes atherosclerotic plaque rupture ${ }^{[176]}$. Complex fibrotic plaques are produced as a result of lysis of foam cells, migration and proliferation of vascular smooth muscle cells (VSMCs), and persistent inflammatory response $\mathrm{e}^{[161]}$.

Foamy macrophages bearing numerous lipid droplets are mainly composed of CEs and triglycerides ${ }^{[6]}$. These cells secrete various biologically active molecules and promote vascular remodeling with lipid deposition, eventually resulting in pathological vascular events, including myocardial infarction and cerebral ischemia ${ }^{[176,179,180]}$. Low density lipoprotein (LDL) is the major cholesterol carrier in the blood and continuous uptake of native and denaturated LDLs by macrophages occurs in the subendothelial layer of the arterial intima $^{[179]}$.

Important role of ACAT1 in foam cells formation and development of atherosclerotic lesion

Three enzymes are thought to be responsible for the synthesis of plasma CEs, i.e. ACAT1, ACAT2 ${ }^{[176,181,182]}$, and lectin: cholesterol acyltransferase (LCAT), a glycoprotein that is secreted by the liver into the blood ${ }^{[183]}$. In animals, ACAT1 and ACAT2 were united with LCAT as known sources of CEs ${ }^{[184]}$. In humans, ACAT2 seems to play a much more extensive role than ACAT1 in production of CEs by macrophages because $\mathrm{TgACAT} 2$ induced during intracellular T. gondii infection provide additional enzyme activity that further enhances biogenesis of CEs in the host cells ${ }^{[153,154]}$.

ACAT1 is an essential enzyme in intracellular cholesterol homeostasis and a transmembrane protein localized mainly in the rough endoplasmic reticulum (ER) and perinuclear region that covalently joins excess free cholesterol with fatty acyl-CoA molecule, typically oleoyl-CoA, to form CEs ${ }^{[185]}$. ACAT1 and neutral cholesteryl ester hydrolase regulated cholesterol esterification ${ }^{[2]}$. Esterification causes cholesterol to be moved out of the cell membrane into the cytoplasm where it is stored as lipid droplets ${ }^{[184]}$. The build-up of free cholesterol in membranes can cause membrane deformation, leading to cellular toxicity ${ }^{[186,187]}$. The presence of ACAT1 in the cell membrane limits the plasma membrane cholesterol concentration by inducing cytosolic lipid droplets formation. When cholesterol is in overabundance, such as in arterial macrophages during atherogenesis, ACAT1 may promote enough lipid storage to induce conversion of macrophages to lipid-laden foam cells ${ }^{[184]}$.

ACAT1 is a dominant isoenzyme in monocyte-macrophages ${ }^{[181,188]}$, which plays an essential role in atherosclerotic plaque formation as well as in oxLDL-induced VSMC foam cell build-up ${ }^{[189,190]}$. Expression of ACAT1 and ACAT2 significantly increased after differentiation of monocytes to macrophages ${ }^{[152,191]}$. In macrophages 
and other cell types, ACAT1 overexpression was involved in forming CEs as lipid droplets ${ }^{[191,192]}$, present in the cell cytoplasm ${ }^{[177]}$, and finally in foam cell formation ${ }^{[193,194]}$. Rong et a ${ }^{[195]}$ found an increase in ACAT1 activity after cholesterol loading also in both human and rat aortic SMCs, but ACAT2 was not found to be expressed by arterial SMCs ${ }^{[152]}$. TLR4 has a critical role in triggering inflammation ${ }^{[189]}$ and participates in VSMC activation ${ }^{[196]}$. Unlike the macrophages, VSMCs do not have the proinflammatory properties normally, however, in many atherogenic conditions, inflammatory reaction often was present also in these cells ${ }^{[197]}$. It was demonstrated that TLR4-mediated inflammation has been activated by oxLDL in VSMCs, which in turn upregulated ACAT1 expression and finally contributed to VSMC foam cell formation required in atherosclerotic plaque building ${ }^{[189]}$.

Sakashita et al ${ }^{[198]}$ demonstrated that in normal human tissues ACAT1 was detected in macrophages, smooth muscle cells, antigen-presenting cells, steroid hormone-producing cells, neurons, myenteric ganglia, cardiomyocytes, hepatocytes, mesothelial and other cells, follicles, tubules. The immunohistochemical reactivities were particularly prominent in the macrophages, steroid hormoneproducing cells, followed by hepatocytes, and intestinal epithelia. In cultured human macrophages ACAT1 was located mainly in the tubular ER, and on cholesterol loading, about 30 to $40 \%$ of the total immunoreactivity appeared in small-sized vesicles enriched in $78 \mathrm{kD}$ glucose-regulated protein ${ }^{[198]}$.

Miyazaki et $a l^{[191]}$ examined human atherosclerotic lesions using immunohistochemical staining and showed that in early lesions of the human aorta, mononuclear cells expressed only limited ACAT1. In contrast, the same ACAT1 antibodies stained much more intensely in fatty streak lesions, particularly in areas that contained macrophages with foamy transformation. It was demonstrated that monocytes/ macrophages were the major cellular component of the ACAT1expressing cells in atherosclerotic lesions ${ }^{[191]}$. In animal experiments, cholesterol feeding increased ACAT1 mRNAs by 2- to 3-fold in mouse and rabbit livers and in rabbit aortas ${ }^{[199,200]}$, respectively, suggesting that the ACAT1 message level may be regulated in a tissue specific manner ${ }^{[191]}$.

\section{Factors affecting ACAT1 upregulation or downregulation}

Cheng et $a^{[201]}$ showed that cholesterol itself served as an activator of ACAT1, besides serving as a substrate. The biosignals that upregulated the expressions of ACAT1 in monocyte-derived macrophages included IFN- $\gamma$ (a proatherogenic cytokine), dexamethasone, and vitamin $\mathrm{D}^{[201-203]}$. On the other hand, a synthetic retinoid Am80 (a specific retinoic acid receptor- $\alpha$ agonist which ameliorated various immunological diseases ${ }^{[204]}$ ), reduced the expression of ACAT1 and HMG-CoA, increased diacylglycerol acyltransferase-1(DGAT-1) expression, inhibited T. gondii -induced acquisition and synthesis of cellular cholesterol in macrophage cell line J774.1, suppressed the growth of the parasite, and inhibited IL-6 and atherosclerosis ${ }^{[205,206]}$.

Insulin was shown to enhance ACAT1 expression in THP-1 macrophages $^{[207]}$. Leptin, an adipose tissue-derived hormone, also accelerated CEs accumulation by increasing ACAT1 expression in human monocyte-derived macrophages and cholesterol efflux from these cells ${ }^{[208]}$. Hori et al ${ }^{[209]}$ found that TGF- $\beta 1$, a multifunctional cytokine with important roles in both host defense and immunopathogenesis, increased ACAT1 protein expression and activity by 2 - to 3 -fold during differentiation of human monocytes into macrophages. It was suggested that ACAT1 expression in monocytes infiltrating from the blood circulation to vascular walls may be enhanced by pre-existing increased serum TGF- $\beta 1$ concentrations $^{[209]}$. (Nb. TGF- $\beta$ expression in human retinal pigment epithelial cells enhanced by $T$. gondii may play an important role in the pathogenesis of retinochoroiditis ${ }^{[210]}$ ). Infection with Chlamydia pneumoniae (a pathogen isolated from coronary and carotid atheromatous plaques which plays an important role in cardiovascular disease $^{[211]}$, hides inside apoptotic neutrophils to silently infect and propagate in macrophages ${ }^{[212]}$ ) also induced macrophage-derived foam cell formation by upregulating ACAT1 expression and thus disturbed cholesterol homeostasis in these cells ${ }^{[213,214]}$. At present, one cannot exclude also a pathogenic role of Helicobacter cinaedi (one of the the most common enterohepatic Helicobacter species that causes bacteriemia in humans) infection in promoting atherosclerosis by involving macrophage-driven proinflamatory responses associated with TLR2 and TLR4 signaling, and causing foam cell formation ${ }^{[215]}$.

Interestingly, Zhao et al ${ }^{[216]}$ showed that hydrogen sulfide $\left(\mathrm{H}_{2} \mathrm{~S}\right)$, a novel endogenous gasotransmitter and a well-known toxic gas with a characteristic smell of rotten eggs present also in drinking waters of wells and thermal spring and mineral waters, inhibited human monocyte-derived macrophage foam cell formation by suppressing ACAT1 production. $\mathrm{H}_{2} \mathrm{~S}$ shares similar features with $\mathrm{NO}$, and $\mathrm{CO}$, and play important vasodilatatory and antiatherosclerotic roles ${ }^{[216-218]}$. These cytoprotective actions may at least partly explain $\mathrm{H}_{2} \mathrm{~S}$ beneficial effects in slowing down progression of experimental Alzheimer's disease ${ }^{[219]}$, as well as improving health in major cardiovascular diseases ${ }^{[220]}$. It was recently suggested that atherosclerosis and Alzheimer are diseases with a common cause ${ }^{[221]}$, and it seems that chronic $T$. gondii infection may be at least in part responsible for this causal relationship ${ }^{[222,223]}$.

Crucial role of ACAT2 in foam cells formation and development of atherosclerotic lesion

ACAT2 is expressed only in intestinal enterocytes, hepatocytes (where ACAT1 is silent ${ }^{[224]}$ ), and in macrophages ${ }^{[152,225]}$. Parini et al ${ }^{[226]}$ found that ACAT2 protein expression was localized to hepatocytes as the major cholesterol-esterifying enzyme in human liver, whereas ACAT1 was present in Kupfer cells only. Human fetal liver (20 weeks post-conception) had abundance of ACAT2 mRNA $^{[227]}$, and in congenital hyperlipidemic mice ACAT2 was present in lipidladen cells of the atherosclerotic plaques ${ }^{[152]}$. It must be noted that the ACAT2 protein expressed in Chinese hamster ovary cell had a halflife $>6 \mathrm{hrs}$, whereas the ACAT1 protein had a $\mathrm{t}_{1 / 2}$ of only $\cong 20-30$ min, suggesting greater stability of ACAT2 ${ }^{[226]}$.

ACAT2 participates in: (a) the enterohepatic recirculation of cholesterol, as revealed by the reduced intestinal absorption efficiency in ACAT2-deficient mice ${ }^{[228]}$; (b) in regulation of cholesterol metabolism within the hepatocyte; and (c) in CE secretion and transport in plasma lipoproteins ${ }^{[184]}$.

ACAT2 functions in free cholesterol esterification during cholesterol absorption in the enterocyte ${ }^{[224]}$. This is a key cholesterol esterification enzyme necessary for the development of hypercholesterolemia ${ }^{[228]}$. Most of the absorbed cholesterol is esterified by ACAT2 for incorporation into chylomicron particles, so that $75-80 \%$ of newly absorbed cholesterol transported into the body in chylomicrons as $\mathrm{CE}^{[229]}$. The metabolism of chylomicrons in the circulation leads to the formation of remnant lipoproteins that retain the $\mathrm{CE}$, which is ultimately delivered to the hepatocytes in the liver ${ }^{[24]}$. The LCAT derived CE of HDL is removed from plasma primarily by the liver through selective CE uptake ${ }^{[230]}$ and by whole HDL particle uptake ${ }^{[231]}$. Normally, more than $70 \%$ of the cholesterol in LDL and VLDL is esterified, and two enzymes ACAT2 and 
LCAT (a liver-derived plasma protein) responsible for the synthesis of plasma lipoprotein $\mathrm{CEs}^{[232]}$ are important determinants of both LDL and VLDL cholesterol concentrations and the subsequent development of atherosclerosis ${ }^{[233,234]}$. Lee et al ${ }^{[233]}$ established that while ACAT2 contributed CEs to newly secreted VLDL, LCAT added CE during LDL particle formation in mice ${ }^{[224,233]}$. ACAT2 deficiency led to a marked decrease in the percentage of CEs $(37.2 \pm 2.1 \%$ vs $3.9 \pm 0.8 \%)$ in plasma VLDL, with a concomitant increase in the percentage of triglyceride $(33.0 \pm 3.2 \%$ vs $66.7 \pm$ $2.5 \%)^{[233]}$. LDL is the major cholesterol carrier in the blood, and in early stages of atherosclerosis, continuous uptake of naive or denatured LDLs by macrophages occurs in the subendothelial layer of the aortic intima. The build-up of free cholesterol in membranes can cause membrane deformation, leading to cellular toxicity ${ }^{[179,186,187]}$. Plasma CEs provided by ACAT2 and LCAT have opposite atherosclerotic potential ${ }^{[232]}$. Saturated and monounsaturated CEs derived predominantly from ACAT2 were associated with increased atherosclerosis ${ }^{[235]}$, whereas polyunsaturated fatty acids containing CEs derived from LCAT were associated with decreased atherosclerosis $^{[236]}$. LCAT acts primarily on plasma HDL to produce cholesterol linoleate and arachidonate ${ }^{[237]}$, and for example, in patients with coronary heart disease, the percentage of cholesterol linoleate was found to be diminished ${ }^{[238,239]}$. This is in agreement with the finding of Degirolamo et $a l^{[240]}$ that polyunsaturated fat provide atheroprotection, at least in part, because it limited the accumulation of cholesteryl oleate in favor of cholesteryl linoleate in plasma lipoproteins. In addition, several data suggested that the cholesteryl oleate and cholesteryl palmitate synthesized by ACAT2 are particularly atherogenic ${ }^{[233,235,241]}$ presumably because of the limited ability of macrophages to mobilize cholesterol from these $\mathrm{CES}^{[242]}$. It was also proposed that cholesteryl oleate should be used as a biomarker for atherosclerosis ${ }^{[243]}$.

ACAT2 is an ER-localized transmembrane protein that transfers the fatty acyl moiety of acyl-CoA to free cholesterol producing mainly cholesteryl oleate and palmitate, and ACAT2-derived CEs can be packaged directly into nascent apoB-containing lipoproteins or stored as neutral droplets in the cytosol ${ }^{[181,182,226,244,245]}$. Liang et a ${ }^{[246]}$ demonstrated that overexpression of either ACAT1 or ACAT2 in rat hepatoma cells increased the secretion and cellular accumulation of $\mathrm{CE}$ to similar extent, but ACAT2 expression caused a greater increase in apoB secretion than ACAT1. It is believed that the primary role of liver ACAT2 expression, relevant to atherogenesis, was to enrich apoB-containing lipoproteins with cholesteryl oleate and palmitate, as opposed to increasing the number of secreted apoB-containing particles $^{[234]}$. The percentage of lipoprotein cholesteryl oleate and the rate of hepatic cholesteryl oleate secretion were found to be positively related to the extent of atherosclerosis in monkeys ${ }^{[235,247]}$. Temel et $a l^{[234]}$ demonstrated that the relative level of ACAT2 expression in cells affects the CEs content and, hence, the potential atherogenicity, of nascent apoB-containing lipoproteins (Table 14).

Cases et $a l^{[155]}$ demonstrated in H5 insect cells (these cells have very low levels of cellular cholesterol and lack meaningful cholesterol esterification activity ${ }^{[192]}$ ) that of the fatty acids tested, ACAT1 showed a slight preference for oleoyl, while that for ACAT2 was palmitoyl $\geqslant$ linoleoyl $>$ arachidonyl (Table 15). They also found that for ACAT1, palmitoyl, linoleoyl, and to a lesser extent, arachidonyl, competed with oleoyl for incorporation into CEs, while for ACAT2, both linoleoyl and palmitoyl competed with oleoyl for incorporation into these structures (Table 16). Moreover, it was established that ACAT1 had 58\% higher activity with oleoyl-CoA compared with palmitoyl-CoA, while ACAT2 showed only small
Table 14 Effect of ACAT2 on the radiolabelled lipid composition of apoB34-containing lipoproteins (acc. to Temel et al ${ }^{[234]}$; with own modification).

\begin{tabular}{|l|c|c|c|c|c|}
\hline \multirow{2}{*}{ ACAT2 Plasmid $(\mu \mathrm{g})$} & \multicolumn{5}{|c|}{ Percentage total $\left[^{3} \mathrm{H}\right]$ lipid } \\
\cline { 2 - 6 } & PL & TG & CE & CE/TG & PL/CE+TG \\
\hline 0 & 19.1 & 77.7 & 3.4 & 0.042 & 0.24 \\
\hline 1 & 19.2 & 68.7 & 14.5 & 0.211 & 0.2 \\
\hline 2.5 & 19.0 & 63.5 & 17.4 & 0.274 & 0.24 \\
\hline 5 & 20.3 & 57.8 & 22.0 & 0.38 & 0.25 \\
\hline
\end{tabular}

Values shown are means of duplicate samples. ApoB, apolipoprotein B, CE, cholesteryl ester; PL, phospholipids; TG, triglyceride.

Table 15 Fatty acyl-CoA specificity for cholesterol esterification by ACAT1 and ACAT2 expressed in $\mathrm{H} 5$ cells. Esterification of $\left[{ }^{14} \mathrm{C}\right]$ cholesterol using different fatty acyl-CoAs as substrates (acc. to Cases et $a l^{[155]}$; with own modification).

\begin{tabular}{|l|c|c|c|c|}
\hline Experiment & $\begin{array}{c}\text { Relative } \\
\text { Oleoyl-CoA }\end{array}$ & $\begin{array}{c}\text { activities (units) } \\
\text { Palmitoyl-CoA }\end{array}$ & $\begin{array}{c}\text { Linoleoyl- } \\
\text { CoA }\end{array}$ & $\begin{array}{c}\text { Arachidonyl- } \\
\text { CoA }\end{array}$ \\
\hline ACAT1 \\
\hline Experiment 1 & 1.0 & 0.9 & 0.7 & 0.4 \\
\hline Experiment 2 & 1.0 & 0.6 & 0.7 & 0.8 \\
\hline ACAT2 & 1.4 & 1.4 & 0.6 \\
\hline Experiment 1 & 1.0 & 1.7 & 1.4 & 0.7 \\
\hline Experiment 2 & 1.0 & 1.4 &
\end{tabular}

Cell membranes were prepared and assayed in triplicate. The cholesterol: phospholipid liposome molar ratio was 0.2 . For ACAT1, the values with oleoyl-CoA were 566 and $499 \mathrm{pM}$ of cholesterol esters formed/mg protein/min for experiments 1 and 2, respectively, and for ACAT2, they were 594 and $529 \mathrm{pM}$ of cholesterol esters formed per $\mathrm{mg}$ of protein $/ \mathrm{min}$.

Table 16 Fatty acyl-CoA specificity for cholesterol esterification by ACAT1 and ACAT2 expressed in H5 cells. Competition of fatty acylCoAs with $\left[{ }^{14} \mathrm{C}\right]$ oleoyl-CoA for cholesterol esterification (acc. to Cases et $a l^{[155]}$; with own modification)

\begin{tabular}{|l|c|c|c|c|}
\hline Experiment & $\begin{array}{c}\text { Relative } \\
\text { Oleoyl-CoA }\end{array}$ & $\begin{array}{c}\text { activities (units) } \\
\text { Palmitoyl-CoA }\end{array}$ & $\begin{array}{c}\text { Linoleoyl- } \\
\text { CoA }\end{array}$ & $\begin{array}{c}\text { Arachidonyl- } \\
\text { CoA }\end{array}$ \\
\hline ACAT1 \\
\hline Experiment 1 & 1.0 & 0.7 & 0.8 & 0.9 \\
\hline Experiment 2 & 1.0 & 0.8 & 0.8 & 0.8 \\
\hline ACAT2 & 0.8 & 0.7 & 1.3 \\
\hline Experiment 1 & 1.0 & 0.8 & 0.8 & 1.3 \\
\hline Experiment 2 & 1.0 & 0.8 &
\end{tabular}

Cell membranes were prepared and assayed in triplicate. The cholesterol: phospholipid liposome molar ratio was 0.7. For ACAT1, the values with oleoyl-CoA were 749 and $644 \mathrm{pM}$ of cholesterol esters formed/mg protein/min for experiments 1 and 2, respectively, and for ACAT2, they were 1661 and $1590 \mathrm{pM}$ of cholesterol esters formed per mg of protein/ min.

Table 17 Fatty acyl-CoA specificity for cholesterol esterification by ACAT1 and ACAT2 expressed in H5 cells. Comparison of cholesterol esterification using $\left[{ }^{14} \mathrm{C}\right]$ oleoyl-CoA or $\left[{ }^{14} \mathrm{C}\right]$ palmitoyl-CoA (acc. to Cases et $a l^{[155]}$; with own modification).

\begin{tabular}{|l|c|c|}
\hline Experiment & $\begin{array}{c}{\left[{ }^{14} \mathrm{C}\right] \text { Oleoyl-CoA pM cholesterol }} \\
\text { ester/mg protein/min }\end{array}$ & $\begin{array}{c}{\left[{ }^{14} \mathrm{C}\right] \text { Palmitoyl- }} \\
\text { CoA }\end{array}$ \\
\hline ACAT1 & 529 & 334 \\
\hline ACAT2 & 1823 & 1573 \\
\hline
\end{tabular}

Cell membranes were prepared and assayed in triplicate. Values were \pm $<5 \%$ in all cases. The cholesterol:phospholipids liposome molar ratio was 0.7 .

difference (Table 17) ${ }^{[155]}$. All these data strongly suggested that ACAT2 play a much more extensive role than ACAT1 in generation of CEs by macrophages ${ }^{[155]}$. Miller et a ${ }^{[248]}$ proposed that CEs 
associated with ACAT2 may have a predictive value in diagnosis of coronary artery disease in patients with acute chest pain.

Further studies of Seo et $a l^{[190]}$ also demonstrated that incubation of oleic acid, arachidonic acid, and eicosapentaenoic acid induced ACAT1 mRNA levels 1.5-2-fold in HepG2 cells, with no effect on ACAT2 mRNA. Both HepG2 and THP1 cells showed the greatest CE production with oleic acid. ACAT1 expressing cells indicated a strong preference for oleic acid while ACAT2 expressing cells utilized unsaturated free fatty acids (FFAs). THP1 and ACAT1 expressing cells utilized oleoyl CoA preferentially. In contrast, HepG2 and ACAT2 microsomes utilized linolenoyl CoA as well. The authors believed that FFAs increased ACAT1 mRNA levels in a cell specific manner, and that the ACAT reactions exhibited differential FFA utilization ${ }^{[190]}$

\section{Factors affecting ACAT2 downregulation}

Estrogen have beneficial effects on plasma lipids and lipoproteins, and reduced atherosclerosis progression ${ }^{[249,250]}$. Kavanagh et al ${ }^{[250]}$ found that estrogen decreased atherosclerosis primarily by reducing hepatic ACAT2 activity in ovariectomized monkeys. Estrogen regulation of enzymatic activity was established at the protein level as both ACAT1 and ACAT2 protein (but not mRNA levels), were lower $(p=0.02 \text { and }<0.0001 \text {, respectively })^{[250]}$. Interestingly, hepatic microsomal ACAT2 activity in normolipidemic, non-obese Chinese females was significantly lower than observed in males ${ }^{[251]}$. Moreover, the activity of ACAT2 correlated negatively with plasma levels of HDL-C $(r=-0.57, p<0.05)$ and with Apo AI $(r=-0.49, p<0.05)$. The observed sex-related difference may thus contribute to female protection from complications of coronary heart disease ${ }^{[251]}$. Saba et $a l^{[252]}$ showed that black ginseng extracts (Ginseng Radix nigra, a well-characterized medicinal herb with antioxidant activity and increased radical scavenging properties) significantly downregulated ACAT2 and HMG-CoA mRNA expressions in murine hepatocytes. Chronic fluticasone propionate (a glucocorticoid) exposure also decreased ACAT2 gene expression in human monocytes and macrophages ${ }^{[253]}$ ). Orange juice or hesperidin consumption resulted in a decrease in the expression of genes encoding ACAT2 together with an increase in the expression of genes regulating reverse cholesterol transport ${ }^{[254]}$. ACAT2 was also downregulated by naringenin, a flavonoid from grapefruit, which could reduce foam cell formation ${ }^{[254,255]}$. Interestingly, curcumin (no effect on ACAT1 or ACAT2; inhibition of oxLDL uptake and promotion of cholesterol efflux from macrophages) ${ }^{[256,257]}$ and erythropoietin (increased cholesterol efflux from macrophages) ${ }^{[258]}$, were reported to suppress the formation of macrophage foam cells. Finally, it should be noted that delta 9-tetrahydrocannabinol (THC), the main constituent of marijuana (Cannabis sativa), administered by a noninhalation route may exert dose-dependent $(1 \mathrm{mg} / \mathrm{kg} / \mathrm{day}$; low $0.6 \mathrm{ng} / \mathrm{mL}$ plasma levels with no psychotropic effects) atheroprotective effects via its immunomodulatory and antiinflammatory actions ${ }^{[259]}$, as well as inhibition of ACAT1 and ACAT2 activities ${ }^{[260]}$.

In summary, ACAT2 enzyme has a longer half-life than ACAT1. For ACAT2, both linoleoyl and palmitoyl competed with oleoyl for incorporation into CEs, while for ACAT1, palmitoyl, linoleoyl, competed with oleoyl for this biochemical reaction. ACAT1 had a higher activity with oleoyl-CoA compared with palmitoylCoA, while ACAT2 showed only small difference. All these data strongly suggest that ACAT2 play a much more extensive role than ACAT1 in generation of CEs by macrophages, and thus in foam cells biogenesis. Clinically, it was proposed that CEs associated with ACAT2 may predict coronary artery disease in patients with acute coronary syndrome, especially that gender-related differences in hepatic microsomal ACAT2 activity have been documented in normolipidemic, non-obese Chinese females.

\section{IMPORTANT ROLE OF TOLL-LIKE RECEPTORS (TLRS), ESPECIALLY TLR2 AND TLR4, IN BOTH T. gondii INFECTION AND DEVELOPMENT OF ATHEROSCLEROSIS}

\section{Atherosclerosis}

The first steps of atherosclerosis are inflammatory in nature ${ }^{[40,261]}$. A family of TLRs has been defined as a key component of pathogenassociated molecular pattern recognition machinery ${ }^{[262,263]}$. TLRs are proinflammatory sensors of pathogens, and potential links between infectious disease, inflammation and atherosclerosis, originally identified as receptors that activate host defenses in response to microbial-derived ligands such as Gram-negative bacterial lipopolysaccharides (LPS) ${ }^{[264,265]}$, and T. gondii ${ }^{[266,267]}$. There are growing evidence showing the contribution of the TLR-signaling pathway to initiation and progression of atherosclerosis ${ }^{[268,269]}$. $\mathrm{Xi}$ et $a l^{[270]}$ performed a systemic analysis of the molecular pathomechanism underlying atherosclerosis and found that 1312 genes from 45,304 publications, were associated with atherosclerosis. 35 gene ontology terms were significantly enriched, and the most highly overrepresented pathway went to the TLR signaling pathway, known to play an important role in atherosclerosis in both inflammatory and immune response ${ }^{[270]}$.

Two prominent risk factors, infectious disease and hyperlipidemia point to innate immune mechanisms as potential contributors to proatherogenic inflammation ${ }^{[271]}$. Atherosclerotic lesions form only in arteries at sites of disturbed blood flow ${ }^{[272]}$. Although all endothelial cells are exposed to comparable levels of circulating plasma cholesterol, only endothelial cells overlaying lesions display an inflamed phenotype ${ }^{[75]}$. This occurs even in the absence of any additional exacerbating disease factors because blood flow controls the expression of TLRs which are initiators of cellular activation and inflammation. TLR2 and TLR4 expression exerted an overall proatherogenic effect in hyperlipidemic mice. TLR activation of the endothelium promoted lipid and leukocyte accumulation within lesions ${ }^{[75]}$.

TLRs ligands are expressed in various cell types within the atherosclerotic plaque ${ }^{[273]}$. TLR1, TLR2, and TLR4 were upregulated in human atheroma with active NF- $\mathrm{BB}$ colocalizing with TLR2 and TLR4 in the plaque ${ }^{[263]}$. Tobias and Curtis ${ }^{[271]}$ observed that proatherogenic TRL2 responses to so far unknown endogenous or unknown endemic exogenous agonists were mediated by non bonemarrow-derived cells, including endothelial cells. TLR ligands potentially associated with development of atherosclerosis are presented in Table 18.

TLR-2 ligands (lipoproteins, such as peptidoglycan and lipoteichoic acids from Gram-positive bacteria) and TLR-4 ligands (LPS from Gram-negative bacteria) play an important role in the innate and inflammatory signaling to activate the NF- $\kappa \mathrm{B}$ pathway and release of proinflammatory cytokines ${ }^{[276-283]}$. Activation of NF- $\kappa B$ is essential for the regulation of a variety of genes involved in the inflammatory and proliferative responses of cells critical to atherogenesis ${ }^{[28,285]}$. Edfeldt et $a l^{[263]}$ found that the expression of TLR1, TLR2 (peptidoglycan as a ligand), and TLR4 (LPS as a ligand) was markedly enhanced in human atherosclerotic plaques and associated with inflammatory activation of endothelial cells and macrophages. TLR expression 
by macrophages permitted local differentiation of these cells into antigen-presenting cells because TLR2 ligation stimulated this process $^{[286]}$. Therefore, TLRs may provide an important link between local innate and adaptive immunity and enhance the cellular immune response in the plaque to local antigens such as heat shock proteins (HSPs) and oxidized lipoproteins ${ }^{[263]}$.

In the advanced atherosclerotic lesions in humans, TLR1, TLR2, TLR4 were detected in macrophages, endothelial cells, and vascular smooth muscle cells (VSMCs), while TLR2 and TLR4 were expressed in atherosclerotic lesion ${ }^{[263,264,280]}$. Human VSMCs were reported to express functional TLR2 and TLR4 signal complexes linked to chemokine and proinflammatory cytokine release ${ }^{[287]}$. The expressions of TLR2 and TLR4 in VSMCs were augmented in the atherosclerotic lesions ${ }^{[288]}$. Local overexpression of TLR-like receptors at the vessel wall induced atherosclerotic lesion formation. Shinohara et a $a^{[264]}$ demonstrated that the expression of both TLR2 and TLR4 at the vessel wall, particularly in VMSCs, synergistically accelerated atherosclerotic process at its early stage, especially under hypercholesterolemia. They also showed that overexpression of TLR2 and TLR4 in VMSCs induced the synergistic activation of NF$\mathrm{\kappa B}$ at the vessel wall, which resulted in augmented expression of key molecules of atherosclerosis such as intracellular adhesion molecule 1 , vascular cell adhesion molecule 1 , and monocyte chemoattractant protein-1 ${ }^{[264]}$.

$\mathrm{Xu}$ et $a l^{[280]}$ showed that the proinflammatory signaling receptor TLR4 colocalized with lipid-rich and macrophage-infiltrated murine atherosclerotic lesions and at the shoulder region of human coronary artery plaques. Furthermore, basal TLR4 mRNA expression by cultured human monocyte-derived macrophages was upregulated by oxLDL (but not native LDL) in vitro, raising the possiblity that enhanced TLR4 expression may play a role in the inflammatory responses linking lipids to chronic infection, inflammation, and atherosclerosis $^{[40,289,290]}$. In fact, TLR4 was expressed by macrophages in murine and human lipid-rich atherosclerotic plaques and upregulated by oxLDL ${ }^{[280]}$.

HSP60 was found to be implicated in the pathogenesis of atherosclerosis ${ }^{[291]}$, and the intensity of its expression correlated positively with the atherosclerotic severity ${ }^{[292,293]}$. Hypoxia induced HSP expression in human coronary artery bypass grafts ${ }^{[36]}$. Berberian et $a l^{[294]}$ reported also the elevated expression of HSP70 in human atherosclerotic plaques, and it was mainly concentrated in the central portions of more thickened atheromas around sites of necrosis and lipid accumulation ${ }^{[25]}$. OxLDL triggered in vitro the expression of HSP60 in monocytes/macrophages ${ }^{[296]}$, and HSP70 in human endothelial cells ${ }^{[297]}$ and SMCs ${ }^{[298]}$. It appeared that oxLDL induced HSP70 expression mainly in growing endothelial cells, indicating that those cells, present in lesion-prone sites, were more sensitive to oxLDL than were quiescent cells ${ }^{[299]}$. HSP70 played a major role in cytokine generation in human oxLDL treated macrophages ${ }^{[300]}$, and it was demonstrated that plasma HSP70 levels predicted risk of acute coronary syndrome ${ }^{[301]}$, the development of atherosclerosis in patients with established hypertension ${ }^{[302]}$, and had atheroprotective properties $^{[303]}$.

Gonzalez-Ramos et al ${ }^{[304]}$ found that HSP70 increased extracellular matrix (ECM) production by human VSMCs through transforming growth factor- $\beta 1$ (TGF- $\beta 1$ ) up-regulation. TGF- $\beta 1$ is a vascular profibrotic cytokine that is regulated in part by activator protein 1 (AP-1)-dependent transcriptional mechanisms. HSP70 can interact with the VSMCs, the major producer of ECM proteins, through the TLR4. The authors demonstrated that extracellular HSP70 binds to human aorta smooth muscle cell TLR4, which upregulated the
Table 18 TLR ligands implicated in atherosclerosis (acc. to Hodgkinson \& $\mathrm{Ye}^{[273]}$; with own modification).

\begin{tabular}{|l|c|}
\hline TLR & Ligand \\
\hline TLR2 & $\begin{array}{c}\text { Oxidized LDL, hyaluronan, HSP60, peptidoglycan, Apo CIII, } \\
\text { HMGB1 }\end{array}$ \\
\hline TLR3/9 & CMV, viral dsDNA \\
\hline TLR4 & $\begin{array}{c}\text { LPS, modified LDL, fibrinogen, hyaluronan, heparan sulfate, } \\
\text { fibronectin EDA, HSP60, tenascin C, } \beta \text {-defensin 2, HMGB1 }\end{array}$ \\
\hline
\end{tabular}

Apo CIII, apolipoprotein CIII (Apo CIII induces expression of VCAM-1 in vascular endothelial cells and increases adhesion of monocytic cells); CMV, cytomegalovirus; dsDNA, double stranded DNA; fibronectin EDA, fibronectin extra domain A; HMGB1, high-mobility group box protein 1 (a proinflammatory molecule); LPS, lipopolysacharide. Stimulation with heparan sulfate induced dendritic cells (DCs) to secrete proinflammatory cytokines, such as TNF-a and IL-6, an effect that was TLR4 dependent ${ }^{[273,274]}$. DCs phenotypically and functionally matured in response to heparan sulfate, an effect that was abrogated when TLR4 was inhibited $^{[275]}$

AP-1-dependent transcriptional activity of the TGF- $\beta 1$ promoter. The TGF- $\beta 1$ upregulation increased the expression of the ECM proteins type I collagen and fibronectin. These findings may help in elucidating the pathomechanisms by which HSP70 contributed in the inflammation and fibrosis observed in atherosclerosis ${ }^{[304]}$.

\section{T. gondii infection}

TLRs expressed differentially among immune cells ${ }^{[305-307]}$ recognize microbial components distinct from host self molecules. Glycosylphosphatidylinositol (GPI)-anchored proteins dominate the surface of the $T$. gondii tachyzoite ${ }^{[308,309]}$, and GPI synthesis is an essential process for viability of the parasite ${ }^{[310,311]}$. GPI from tachyzoites induced TNF- $\alpha$ generation in macrophages via the activation of the transcription factor NF-kB ${ }^{[312]}$. Debierre-Grockiego et $a l^{[307,311,312]}$ suggested that both TLR2 and TLR4 receptors may contribute in collaboration with other MyD88-dependent receptors (e.g. TLR9) in the host defense against $T$. gondii infection through their activations by the GPIs. Interestingly, the native core glycans and the diacylglycerols isolated from purified T. gondii GPIs also were able to activate both TLR2 and TLR4, and simultaneous deficiency of TLR2 and TLR4 was required to abrogate the in vitro TNF- $\alpha$ production by macrophages exposed to T. gondii GPIs ${ }^{[307]}$.

Denkers et $a l^{[313,314]}$ found that macrophages, which along with DCs are targets of in vivo infection, produced cytokines such as TNF- $\alpha$ and IL-12 when activated through TLR4 on the host cell surface $^{[315,316]}$. If these cells were first exposed to tachyzoites, they lost their ability to respond to IFN- $\gamma$, and TNF- $\alpha$ production was suppressed. Because other biomediators such as antiinflammatory IL-10 were unaffected, carrying the parasite did not make such cells generally dysfunctional. Moreover, only cells containing intracellular pathogens became unresponsive and there was no bystander effect on uninfected cells. Instead, $T$. gondii directly subverted these signaling pathways within host cells probably as a defense mechanism to avoid or delay induction of antimicrobial activity and/or T-cell-mediated immunity during host infection ${ }^{[316,317]}$.

T. gondii-derived HSP70 stimulated maturation of murine marrowderived DCs through TLR ${ }^{[60]}$. Under stress conditions, T. gondii RH strain tachyzoites differentiated into bradyzoites and induced $T$. gondii HSP70 ( $\mathrm{TgHSP} 70$ ) protein expression, a highly immunogenic protein $^{[318-321]}$. Also, reactivation $T g H S P 70$ in vivo induced expression of $T g$ HSP70 during bradyzoite to tachyzoite interconversion ${ }^{[322]}$. Recombinant (rTgHSP70) and natural TgHSP70 stimulated the NO release by peritoneal macrophages via TRL2, MyD888 and IRAK4, but under restimulation, signaling of $\mathrm{r} T g \mathrm{HSP} 70$-induced tolerance 
was mediated by TRL4 $4^{[323]}$.

HSP70 has been shown to be a major immunomodulant antigen in parasitic and bacterial infections, and the preferred target of humoral and cell-mediated immune responses to infection ${ }^{[320,323-326]}$. Barenco et $a{ }^{[321]}$ found that in mice the $\mathrm{TgHSP} 70$ release into the bloodstream was dependent on the death of $T$. gondii tachyzoites mediated by the host immune response, whereas the increased $\operatorname{TgHSP} 70$ expression in the brain depended on the multiplication rate of the parasite. Aosai et al ${ }^{[327]}$ showed that $T g \mathrm{HSP} 70$ induced prominent responses in murine B cells derived not only from $T$. gondii-infected but also from uninfected mice. B220 $0^{+}$spleen cells showed marked proliferation after stimulation with $\mathrm{TgHSP} 70$ but neither $\mathrm{CD} 4^{+}$nor $\mathrm{CD} 8^{+}$population responded. These data indicated that $\mathrm{TgHSP} 70$ induced proliferation of B cells but not T cells, and TLR4 was found to be required for $\operatorname{TgHSP} 70$-induced B cell activation ${ }^{[189]}$. It should be emphasized that TLR4-mediated inflammation promoted foam cell formation of VSMC by upregulating ACAT1 expression ${ }^{[189]}$.

$\operatorname{TgHSP} 70$ was shown to deteriorate the host defense by downregulating NO release of peritoneal macrophages in the $T$. gondiiinfected host ${ }^{[328]}$. $T g$ HSP70 induced anti-mHSP70 autoantibody formation by B-1 cells in $T$. gondii-infected mice ${ }^{[329]}$. It was reported that HSP70 induced DC maturation after binding to immature DC and suggested the abundant receptor expression for HSP70 on immature $\mathrm{DC}^{[317,330]}$. It is well known that TRL4 is a critical receptor and signal transducer for LPS. LPS downregulated surface expression of the TRL4-MD2 complex on peritoneal macrophages ${ }^{[331,332]}$. It was demonstrated that both $\mathrm{TgHSP} 70$ - and LPS-induced proliferative responses of spleen cells required TRL4 molecules as a receptor ${ }^{[327]}$.

The mechanisms responsible for the atherogenic modification of LDL may include: oxidation mediated by NOS, myeloperoxidase, and/or 15-lipoxygenase $\mathrm{e}^{[333,334]}$. NO is a potent oxidant produced by both endothelial cells and macrophages that appear to exert both protective and atherogenic effects, depending on the source of production. NO produced by endothelial NOS has vasodilator function and is potentially atheroprotective. In contrast, $\mathrm{NO}$ produced via the much higher capacity iNOS in macrophages, serving antimicrobial functions based on its potent oxidative properties, is potentially atherogenic ${ }^{[334]}$.

$\mathrm{NO}$ and its metabolites control a number of host defense functions regulated by activated macrophages, including antimicrobial and tumoricidal activities, implicated in the pathogenesis of tissue damage associated with acute and chronic inflammation ${ }^{[335-337]}$. It was demonstrated that NO lead to the induction of HSP70 protein and mRNA in cultured SMCs ${ }^{[338]}$ and other cells $s^{[339,340]}$. Mun et al ${ }^{[323]}$ found that $T g \mathrm{HSP} 70$-induced NO release was dependent on the TLR2/MyD88 signal transduction pathway, and that prior exposure to $T g \mathrm{HSP} 70$ induced a tolerance of NO production for subsequent stimulation with $T g \mathrm{HSP} 70$ via TRL4 (Table 19). $T g \mathrm{HSP} 70$ was shown to cause deterioration of the host defense by downregulating NO release by peritoneal macrophages in the $T$. gondii-infected $\operatorname{host}^{[323,329,341]}$.

Together, TLR-like receptors play a critical role in the innate immune responses to microbial-derived ligands, including those of $T$. gondii. TLR2 and TLR4 ligands are important for the inflammatory signaling to activate the NF- $\mathrm{kB}$ pathway and release proinflammatory cytokines. In the advanced atherosclerotic lesions in humans, TLR1, TLR2, TLR4 were detected in macrophages, endothelial cells, and VSMCs, while TLR2 and TLR4 were expressed in atherosclerotic lesions. TLR4-mediated inflammation promoted foam cell formation of VSMC by upregulating ACAT 1 expression. Activation of TLR2 and TLR4 by GPIs derived from $T$. gondii associated with the increased production of proinflammatory cytokines, oxLDL, HSP60, HSP70, and NO and its metabolites are all implicated in the pathogenesis of atherosclerosis.

\section{PROINFLAMMATORY CYTOKINES IN - CREASE FOAM CELL FORMATION. LATENT CHRONIC T. gOndii INFECTION PERSIS- TENTLY GENERATES PROINFLAMMATORY CYTOKINES AND THUS PLAY A CRUCIAL ROLE IN FOAM CELL BIOGENESIS}

\section{Atherosclerosis}

Inflammation is a triggering and an aggravating factor in atherogenesis ${ }^{[40,161]}$. Elevated plasma levels of proinflammatory cytokines have been demonstrated in patients with established atherosclerotic disease ${ }^{[162,163]}$. It is therefore believed that cytokines are key players in all stages of disease and have a profound influence on the pathogenesis of the disease $\mathrm{s}^{[342]}$.

TNF- $\alpha$, a proinflammatory cytokine, participates in every step of the inflammation process ${ }^{[159,340-343]}$. High concentrations of TNF- $\alpha$ were found in atherosclerotic lesions, and it was reported that this cytokine regulated multiple genes involved in various stages of atherosclerosis, exhibiting mostly proatherosclerotic properties ${ }^{[159]}$. In animal models of atherosclerosis, inhibition of TNF- $\alpha$ reduced the progression of this process ${ }^{[347]}$. In healthy males, plasma TNF- $\alpha$ concentrations correlated with early carotid artery atherosclerosis ${ }^{[348]}$. TNF- $\alpha$ is secreted by activated macrophages, lymphocytes, endothelial cells, and $\mathrm{SMCs}^{[349]}$. Lei et a ${ }^{[159]}$ showed that in cultured differentiating human monocytes, TNF- $\alpha$ even at low concentrations (2.5-5 ng/mL), increased the expression of the ACAT1 gene, enhanced the CEs accumulation, and promoted foam cells formation.

Cytokine regulation of ACAT1 gene expression in monocytes/ macrophages play important roles in the initiation and progression of atherosclerosis ${ }^{[159]}$. The expression of the human ACAT1 gene was also synergistically upregulated by IL- $1 \beta^{[160,161]}$, IFN- $\gamma^{[203,347]}$, and TGF- $\beta^{[210]}$. In contrast, the human ACAT1 gene expression was downregulated by adiponectin, an adipocytokine that exerted many antiatherosclerotic effects in cell culture studies ${ }^{[350]}$, for example by increasing cholesterol efflux from macrophages ${ }^{[351]}$.

Lipid loading of primary human macrophages resulted in altered cytokine secretion from cells and effects were similar regardless of neutral lipid composition of cells ${ }^{[352]}$. It was demonstrated that formation of triglyceride (TG)-loaded human monocyte-derived macrophages caused a 4-fold increase in basal IL-1 $\beta$ secretion, and

Table 19 Tolerance of NO synthesis by secondary challenge with TgHSP70 through TLR4 (acc. to Mun et al ${ }^{[323]}$; with own modification).

\begin{tabular}{|l|c|c|c|}
\hline Mouse strain & Stimulation & NO synthesis & $\begin{array}{c}\text { ' } \\
\text { Tolerance } \\
\text { induction }^{\text {b }}\end{array}$ \\
\hline \multirow{2}{*}{ Wild type } & TgHSP70 & + & + \\
\cline { 2 - 4 } & LPS & + & + \\
\hline \multirow{2}{*}{ TLR4 deficient } & TgHSP70 & + & - \\
\cline { 2 - 4 } & LPS & - & ND \\
\hline \multirow{2}{*}{ TLR2 deficient } & TgHSP70 & - & ND \\
\cline { 2 - 4 } & LPS & + & + \\
\hline \multirow{2}{*}{ MyD88 deficient } & TgHSP70 & - & ND \\
\cline { 2 - 4 } & LPS & - & ND \\
\hline
\end{tabular}

$\mathrm{a}+, \mathrm{NO}$ synthesis positive; -, NO synthesis negative; $\mathrm{b}+$, occurrence of tolerance induction; -, no occurrence of tolerance induction. ND, not detected, NO synthesis did not occur by primary stimulation with TgHSP70 and LPS. LPS, lipopolysaccharide. TLR2 regulated TgHSP70induced TNF- $\alpha$ mRNA expression and tolerance. 
in contrast, secretion of TNF- $\alpha$ and IL- 6 decreased significantly following both TG and cholesterol loading ${ }^{[352]}$. Formation of TG loaded foam macrophages by incubation with VLDL also resulted in a marked increase in IL-1 $\beta$ expression in these cells ${ }^{[353]}$. Ruan et $a l^{[161]}$ showed that IL-1 $\beta(5-20 \mathrm{ng} / \mathrm{mL})$ enhanced transformation of VSMCs into foam cells by increasing uptake of unmodified LDL via LDL receptors and by enhancing cholesterol esterification. It was found that IL- $1 \beta$ increased both ACAT 1 protein activity and mRNA expression. Exposure to IL-1 $\beta$ also caused overexpression of the sterol regulatory element binding protein (SREBP), cleavageactivating protein, and enhanced its translocation from the endoplasmic reticulum to the Golgi, where it is known to cleave SREBP, thereby enhancing LDL receptor gene expression. Thus, IL$1 \beta$ disrupted cholesterol-mediated LDL receptor feedback regulation, permitting intracellular accumulation of unmodified LDL and causing foam cell formation. This suggested that proinflammatory cytokines may contribute to intracellular accumulation of native LDL without prior modification of the lipoprotein by oxidation or glycosylation under inflammatory stress ${ }^{[161]}$.

IL-33 exerted a controling protective role in atherosclerosis in vivo ${ }^{[354]}$. This IL-1 cytokine family member induced the production of the $\mathrm{T}_{\mathrm{H}} 2$ cytokines IL- 4 and IL-13, and enhanced serum immunoglobulin synthesis ${ }^{[355]}$. IL-33 was a promoter of $\mathrm{T}_{\mathrm{H}} 2$ dependent inflammatory diseases ${ }^{[356,357]}$, and activated a number of cell types, including $\mathrm{T}_{\mathrm{H}} 2$ cells, mast cells and basophils, and promoted systemic sclerosis ${ }^{[358]}$. McLaren et al ${ }^{[354]}$ found that IL33 blocked macrophage foam cell formation in vitro by directly decreasing acetylated LDL and oxLDL uptake, storage of cholesteryl esters and triglyceride, and reducing intracellular total and esterified cholesterol content, and enhancing cholesterol efflux/transport, and by inducing a phenotypical $\mathrm{T}_{\mathrm{H}} 1$-to- $\mathrm{T}_{\mathrm{H}} 2$ switch. IL-33 also decreased the expression of key genes implicated in cholesterol esterification and triglyceride storage, including ACAT1 and adipocyte differentiation-related protein ${ }^{[354]}$.

Feingold et $a l^{[359]}$ found that low doses of LPS stimulated hepatic TG production, while high doses inhibited the clearance of TG-rich lipoproteins in rats. LPS and several cytokines, including TNF- $\alpha$, TNF- $\beta$, IL-1, IL-6, IFN- $\alpha$, platelet activating factor, rapidly induced de novo fatty acid (FA) synthesis and hepatic TG synthesis in rodents (Table 20 ${ }^{[360-362]}$, at least in part by increasing hepatic intracellular concentrations of citrate, an activator of acetyl CoA carboxylase ${ }^{[363]}$. IL-4, an antiinflammatory cytokine, inhibited the stimulatory effects of TNF, IL-1, and IL-6 on hepatic FA synthesis by blocking the increase in hepatic citrate levels ${ }^{[364]}$. LPS stimulated hepatic cholesterol synthesis by increasing the transcription rate, mRNA expression, protein mass, and activity of HMG-CoA reductase ${ }^{[365,366]}$. In rats, LPS significantly inhibited the clearance of LDL from the circulation ${ }^{[367]}$, and decreased the expression of hepatic LDL receptor protein (Table 21)

\section{T. gondii infection}

Acute toxoplasmosis sometimes leads to lethal overproduction of proinflammatory $\mathrm{T}_{\mathrm{H}} 1$ cytokines $^{[33]}$. During acute and chronic infection in mice there were differences in virulence of $T$. gondii and different strains of the parasite induced different cytokine responses $^{[368,369]}$. For example, the serum levels of IL-12, IL-6, IL10 , IFN- $\gamma$, and TNF- $\alpha$ were significantly higher in the type II strain ME49-infected mice than in the FORT-infected mice, suggesting that not all type II strains (most frequent in humans) cause toxoplasma encephalitis in these animals ${ }^{[368]}$. Chronic infection with $T$. gondii induced high levels of the cytokines IL-12 and TNF- $\alpha$, which are typically associated with macrophage function ${ }^{[316]}$, and DCs ${ }^{[370,371]}$ as well as neutrophils ${ }^{[372]}$ also have emerged as essential producers of IL-12. A pyrogenic factor TNF- $\alpha$ is liable to induce the secretion of acute inflammatory phase proteins via the production of IL-6 $6^{[373,374]}$. In toxoplasmosis, TNF- $\alpha$ appeared to be essential for macrophage activation and inhibition of parasite replication, and this action can only be exerted in synergy with IFN- $\gamma^{[373]}$. Macrophages are known as important effectors of the innate immune system, and they posses the potential of directing acquired immunity toward a $\mathrm{T}_{\mathrm{H}} 1$-biased type of immune response $\mathrm{e}^{[375]}$. In addition to producing proinflammatory cytokines such as IL-12, TNF- $\alpha$, IL-1 $\beta^{[32,376-379]}$ and antiinflammatory cytokines (eg, IL-10, TGF- $\beta)^{[32]}$, macrophages are a source of the microbicidal effector molecule NO and are capable of phagocytosing pathogens and degrading them within the phagolysosome. It should however be noted that the parasite also displays potent downregulatory effects on IL-12, TNF- $\alpha$, and NO production by infected macrophages, being a result of tachyzoite-induced blockade of $\mathrm{NF}-\kappa \mathrm{B}$ nuclear translocation ${ }^{[316,380]}$. In addition, $T$. gondii readily uses macrophages as host cells and reside in these cells within a specialized parasitophorous vacuole ${ }^{[381,382]}$, which resists acidification and lysosomal fusion ${ }^{[382]}$.

IL-6 and IL-27 are closely related cytokines that play critical but distinct roles during infection with $T$. gondii, i.e. IL-6 is required for the development of protective immunity to this pathogen, whereas IL-27 is required to limit infection-induced pathology ${ }^{[383]}$. IL-6 is known to mediate both pro- and antiinflammatory effects having two distinct ways to induce cell-signaling: either through the membrane bound receptor (antiinflammatory) or through trans-signaling (proinflammatory ${ }^{[384]}$. This cytokine is the main stimulator of the production of most acute phase proteins ${ }^{[385,386]}$, and is important to the transition between acute and chronic inflammation ${ }^{[387]}$. Moreover, IL-6 played an important role in regulation of the expression of

Table 20 Effects of LPS, lipoteichoic acid (LTA), and cytokines on triglyceride (TG) metabolism in intact rodents (acc. to Khovidhunkit et $a l^{[360]}$; with own modification).

\begin{tabular}{|l|c|c|c|c|c|c|c|}
\hline Parameter & LPS & LTA & TNF & IL-1 & IL-6 & IFN-a & IFN- $\uparrow$ \\
\hline Serum TG & $\uparrow$ & $\uparrow$ & $\uparrow$ & $\uparrow$ & $\uparrow$ & $\leftrightarrow$ & $\leftrightarrow$ \\
\hline $\begin{array}{l}\text { Hepatic FA } \\
\text { synthesis }\end{array}$ & $\uparrow$ & $\uparrow$ & $\uparrow$ & $\uparrow$ & $\uparrow$ & $\uparrow$ & $\leftrightarrow$ \\
\hline FA oxidation & $\downarrow$ & ND & $\downarrow$ & $\downarrow$ & ND & ND & ND \\
\hline TG secretion & $\uparrow$ & $\uparrow$ & $\uparrow$ & $\uparrow$ & $\uparrow$ & ND & ND \\
\hline TG clearance & $\leftrightarrow^{\mathrm{a}}{ }^{\prime} \downarrow^{\mathrm{b}}$ & $\leftrightarrow$ & $\leftrightarrow$ & $\leftrightarrow$ & $\leftrightarrow$ & ND & ND \\
\hline LPL activity & $\downarrow$ & $\leftrightarrow$ & $\downarrow, \leftrightarrow^{\mathrm{c}}$ & $\downarrow$ & $\downarrow$ & $\downarrow$ & $\downarrow$ \\
\hline Lipolysis & $\uparrow$ & $\uparrow$ & $\uparrow$ & $\leftrightarrow$ & $\uparrow$ & $\uparrow$ & $\uparrow$ \\
\hline $\begin{array}{l}\text { Serum ketone } \\
\text { body }\end{array}$ & $\downarrow$ & $\mathrm{ND}$ & $\downarrow$ & $\downarrow$ & $\leftrightarrow$ & $\uparrow^{\mathrm{a}}, \leftrightarrow^{\mathrm{b}}$ & $\uparrow$ \\
\hline
\end{tabular}

FA, fatty acids; LPL, lipoprotein lipase; LPS, lipopolysacchride; ND, not determined. ${ }^{\mathrm{a}}$ Low doses. ${ }^{\mathrm{b}}$ High doses. ${ }^{\mathrm{c}}$ Some but not most tissues.

Table 21 Effects of LPS, lipoteichoic acid (LTA), and cytokines on cholesterol metabolism in intact rodents (acc. to Khovidhunkit et al ${ }^{[360]}$; with own modification).

\begin{tabular}{|l|c|c|c|c|c|c|c|}
\hline Parameter & LPS & LTA & TNF & IL-1 & IL-6 & IFN- & IFN- - \\
\hline Serum cholesterol & $\uparrow, \downarrow^{\mathrm{a}}$ & $\uparrow$ & $\uparrow, \downarrow^{\mathrm{a}}$ & $\uparrow, \leftrightarrow^{\mathrm{a}}$ & $\uparrow$ & $\leftrightarrow$ & $\leftrightarrow$ \\
\hline Hepatic cholesterol synthesis & $\uparrow$ & ND & $\uparrow$ & $\uparrow$ & ND & $\leftrightarrow$ & $\uparrow$ \\
\hline HMG-CoA reductase activity & $\uparrow$ & ND & $\uparrow$ & $\uparrow$ & ND & ND & $\leftrightarrow$ \\
\hline LDL receptor protein & $\downarrow^{\circ} \leftrightarrow^{\mathrm{b}}$ & ND & $\leftrightarrow^{\mathrm{b}}$ & $\leftrightarrow^{\mathrm{b}}$ & ND & ND & ND \\
\hline Bile acid synthesis & $\downarrow$ & ND & $\downarrow$ & $\downarrow$ & ND & ND & ND \\
\hline
\end{tabular}

LDL, low density lipoproteins; HMG-CoA, 3-hydroxy-3-methylglutamyl coenzyme A; LPS, lipopolysacchride; ND, not determined. Primates. ${ }^{\mathrm{b}}$ Hamsters. 
cytokine and chemokine stimulation of antibody production by B cells, regulation of macrophage and DC differentiation, and the response of regulatory $\mathrm{T}$ cells to microbial infection ${ }^{[388-390]}$. This cytokine also protected human macrophages from cellular cholesterol accumulation ${ }^{[391]}$. Most importantly however, because IL-6 was found to promote the intracellular multiplication of T. gondii in mice ${ }^{[392]}$, and in peritoneal macrophages (in a dose-dependent manner) ${ }^{[393]}$, it may play an important role in triggering and development of atherosclerosis.

Lipoprotein-associated phospholipase $\mathrm{A}_{2}\left(\mathrm{Lp}-\mathrm{PLA}_{2}\right)$ is an inflammatory enzyme that catalyzes the hydrolysis of oxidized phospholipids into lysophospholipids on the surface of lipoproteins, and approximately $80 \%$ of serum Lp-PLA $\mathrm{P}_{2}$ was found on the surface of LDL particles, migrating across the vascular endothelial cell layer together with LDL $^{[114]}$. Coronary arterial lesions had a high concentrations of Lp-PLA2 in the necrotic core ${ }^{[394]}$, which suggested its involvement in atherosclerotic plaque rupture ${ }^{[14]}$. Intracellular parasites acquire host cholesterol that endocytosed by the LDL pathway, a process that is specifically increased in $T$. gondii infected host cells ${ }^{[149]}$, and interestingly, $\mathrm{PLA}_{2}$ was found to be implicated in $T$. gondii cell invasion through increasing their penetration ${ }^{[395]}$.

T. gondii RELEASES FROM INFECTED HOST CELLS INTO THE EXTRACELLULAR SPACE SMALL VESICLES KNOWN AS EXOSOMES WHICH CONTAIN PATHOGEN-ASSOCIATED MOLECULAR PATTERNS (PAMP) AND OTHER BIOMOLECULES THAT STIMULATE PROINFLAMMATORY RESPONSES, AND MAY THEREFORE PLAY A ROLE IN THE PATHOGENESIS OF ATHEROSCLEROSIS

Extracellular vesicles (ECVs) are carriers of PAMP and damageassociated molecular patterns (DAMP), cytokines, autoantigens, and tissue-degrading enzymes and have a possible role in pathogenesis of several inflammatory states and various clinical entities, including infections, atherosclerosis, and autoimmune diseases. ECVs contain proteins, lipids, nucleic acids from the cell from which they derived, and participate in intercellular communication ${ }^{[396]}$.

Bhatnagar et al ${ }^{[397]}$ found that THP-1 macrophages infected with intracellular pathogens, including $T$. gondii virulent $\mathrm{RH}$ strain, released from cells small 50-100 nm diameter vesicles, known as exosomes, into the extracellular space. These exosomes, when exposed to uninfected macrophages, stimulated a proinflammatory TNF- $\alpha$ response in a TLR2 or TLR4 receptor- and MyD88-dependent manner, and exosomes, not apoptotic vesicles, were responsible for the proinflammatory activity ${ }^{[397]}$. Exosomes are released, especially during cellular stress, from many different cell types ${ }^{[398]}$, and dendritic cells and B cells expressed MHC class I and II molecules as well as costimulatory particles, and have been shown to promote T-cell activation ${ }^{[399-401]}$. Exosomes from a mouse dendritic cell line incubated with $T$. gondii-derived antigens triggered a strong systemic humoral immune response protective against infection with the pathogen $^{[402]}$. T. gondii infection of human foreskin fibroblasts also caused the production of exosome-like vesicles containing a unique array of mRNA and miRNA transcripts described in the literature data as having abnormal neurologic effects ${ }^{[403-406]}$. The vesicles from infected cells could attach and deliver their contents to uninfected cells, and their RNA contents are not just reflecting a cytoplasmic RNA, thereby causing the function of these cells to be altered ${ }^{[403]}$.

Several stimuli, such as proinflammatory cytokines, lead to ECVs release from monocytes and macrophages in the atherosclerotic lesion ${ }^{[407]}$. ECVs derived from circulating in blood vascular endothelial cells, VSMCs, macrophages, and other circulating immune cells mainly have proinflammatory properties ${ }^{[408]}$, and for example proinflammatory cytokines and NO can trigger blood endothelial cells-derived ECVs production ${ }^{[409]}$. Moreover, recent findings have associated lymphatic dysfunction with the pathogenesis of atherosclerosis, hypercholesterolemia, and cardiovascular disease ${ }^{[410]}$. Inflammatory cell accumulation in peripheral tissues drives several chronic inflammatory diseases, including atherosclerosis and obesity ${ }^{[261]}$ in which lymphatic transport has been found defective ${ }^{[410-412]}$. Milasan et al ${ }^{[413]}$ showed that diverse ECs inclusively derived from erythrocytes and platelets were present in lymph of healthy animals, and their levels were modulated in lymph during atherosclerosis. It must be noted that circulating red blood cells may be infected with $T$. gondii ${ }^{[144]}$, human and chicken thrombocytes have phagocytic capacity ${ }^{[415,416]}$, and adherence of platelets to tachyzoites of the parasite together with disruption of surface membranes and cytoplasmic contents of the organisms, were observed ultrastructurally ${ }^{[417]}$. Although chicken erythrocytes and platelets were found to be infected with the parasite, the pathogen was however not capable of replication in these cells ${ }^{[418]}$.

In summary, the parasite which infect different cell types, participates in the production of many ECVs. Circulating ECVs may contain remnants of $T$. gondii tachyzoites, such as PAMP, DAMP and other microbioparticles that are dispersed to various organs, and may take part in triggering persistent inflammation, lymphatic drainage dysfunction, and development of atherosclerosis and various cardiovascular events.

ANEMIA, HEMORRHAGE, FIBRIN DEPOSI-
TION IN TISSUES, AND DISTURBANCES IN
BLOOD COAGULATION OBSERVED DUR-
ING T. gON III INFECTION MAY WELL EX-
PLAIN SIMILAR ABNORMALITIES CHARAC-
TERISTIC FOR ATHEROSCLEROSIS

In mammals, T. gondii infects all nucleated cells ${ }^{[419]}$, and thus has the potential to directly damage vascular cells, thereby causing hemorrhage and activating a pathophysiological blood coagulation response. Studies of host defense against $T$. gondii infection showed that protection was mediated by a robust proinflammatory $\mathrm{T}_{\mathrm{H}} 1$ type of immune response characterized by the prolific production of large quantities of IFN- $\gamma$ and other proinflammatory cytokines ${ }^{[32,420]}$. Chronic overproduction of IFN- $\gamma$ induced anemia by both reducing mature erythrocyte life span in the circulation and inhibiting erythropoiesis via IL-15 $5^{[421-423]}$. IFN- $\gamma$ also stimulated expression of genes that favored and/or stabilized fibrin deposition ${ }^{[424-426]}$. Del Prete et al ${ }^{[427]}$ documented that $\mathrm{T}_{\mathrm{H}} 1$ and $\mathrm{T}_{\mathrm{H}} 2 \mathrm{~T}$-helper cells exerted opposite regulatory effects on procoagulant activity and tissue factor production by human monocytes.

Several authors reported that blood coagulation leading to fibrin deposition performed multiple protective functions during infection because fibrin: (a) restrained pathogen growth and dissemination, (b) protected against hemorrhagic pathology, and (c) probably promoted tissue repair ${ }^{[428-433]}$. Mullarky et $a l^{[434]}$ found that in mice lacking 
IFN- $\gamma$, IL-12, and/or TNF- $\alpha$ neither hemorrhage nor pathogen burden were responsible for fibrin levels during $T$. gondii infection, and that cytokines exerted dominant regulatory roles (Table 22). It appeared that proinflammatory $\mathrm{T}_{\mathrm{H}} 1$ immunity provoked hemorrhage via IFN- $\gamma$ while independently promoting the deposition of fibrin via TNF- $\alpha^{[434]}$. Earlier studies showed that TFN-a may favor fibrin deposition by stimulating vascular wall permeability ${ }^{[435-437]}$, and the cytokine promoted the formation of fibrin as well as fibrin-associated adhesions and abscesses in a model of septic peritonitis ${ }^{[432]}$. TNF- $\alpha$ also increased expression of procoagulant biomolecules ${ }^{[438,439]}$, including PAI generation ${ }^{[24-26]}$, decreased expression of anticoagulant factors ${ }^{[438-440]}$, and/or suppressed fibrin degradation in human endothelial cells ${ }^{[434,441-443]}$.

Blood coagulation disturbances leading to fibrin deposition frequently accompanied $\mathrm{T}_{\mathrm{H}} 1$ type of immune responses, including autoimmunity ${ }^{[424,429,444-446]}$. Studies using fibrinogen-deficient mice revealed pathological roles for fibrin (ogen) during atherosclerosis ${ }^{[477]}$, and reported that fibrin can upregulate expression of proinflammatory cytokines and chemokines in humans ${ }^{[448]}$. Johnson et al ${ }^{[429]}$ demonstrated that blood coagulation performed important protective function during $T$. gondii infection by suppressing hemorrhage evoked by IFN- $\gamma$, a critical proinflammatory mediator of $T_{H} 1$ immunity. Fibrin deposition mediated protection of host tissue against $T$. gondii infection-stimulated immunopathology ${ }^{[429]}$. These investigators ${ }^{[429]}$ established that increased production of fibrin during T. gondii infection prevented infection-stimulated blood loss, thereby performing a protective function evoked by IFN- $\gamma$, a critical immune mediator elicited by the parasite during host robust $\mathrm{T}_{\mathrm{H}} 1$ type immune response. Since IFN- $\gamma$ and other cytokines stimulated also expression of genes that promote and/or stabilize fibrin deposition ${ }^{[424-426]}$, it may suggest that the enhanced $\mathrm{T}_{\mathrm{H}} 1$ immune response evolved means to locally up-regulate blood coagulation activity, thereby protecting against its own destructive bioprocesses ${ }^{[429]}$.

\section{Plasminogen activator inhibitor-1 (PAI-1)}

PAI-1, a prothrombotic protein and the fast-acting inhibitor of plasminogen activators, is the principal regulator of endogenous fibrinolytic enzyme system. Disruption of the PAI-1 gene was associated with increased resistance to thrombosis and with a mild hyperfibrinolytic state characterized by enhanced in vivo clot $\operatorname{lysis}^{[44,450]}$. Low fibrinolytic capacity has been associated with an increased risk of recurrent major cardiovascular disorders ${ }^{[451]}$. Nilsson et $a l^{[36]}$ showed that unsaturated fatty acids, i.e. oleic, linoleic, and linolenic acid, and eicopentaenoic acid, caused a significant increase in PAI-1 secretion and mRNA levels from cultured human umbilical endothelial and EA.hy926 type cells. Transfection experiments demonstrated that addition of linolenic acid and eicosapentaenoic acid markedly increased PAI-1 transcription ${ }^{[450]}$. It was found that unsaturated fatty acids induced the same complex as did VLDL, whereas saturated fatty acids had no effect. VLDL has been shown to induce a concentration-dependent increase in the PAI-1 secretion from human umbilical venous endothelial cells ${ }^{[452-454]}$ and HepG2 cells in vitro ${ }^{[453,455]}$. In vivo, several studies demonstrated a strong positive correlation between the plasma VLDL triglyceride and PAI1 activity levels ${ }^{[456-458]}$, and a VLDL response element was identified in the promoter region of the PAI-1 gene locus ${ }^{[459]}$. Addition of a triglyceride-rich emulsion also resulted in an enhanced secretion of PAI-1 by HepG2 cells ${ }^{[460]}$. These in vitro data are in line with the finding that administration of $\mathrm{n}-3$ fatty acids in vivo resulted in increased plasma PAI-1 activity ${ }^{[461,462]}$.

The increased level of PAI-1 may critically affect fibrinolysis
Table 22 Dissociation of roles for hemorrhage, pathogen burden, and cytokines in the regulation of fibrin deposition during acute $T$. gondii infection in mice (acc. to Mullarky et al $^{[4344}$; with own modification).

\begin{tabular}{|l|c|c|c|c|c|}
\hline Mouse $^{\mathrm{a}}$ & Fibrin & Hemorrhage $^{\mathrm{b}}$ & Parasites & IFN- $\gamma$ & TNF- $\alpha$ \\
\hline IFN- $\gamma$-deficient & ++++ & - & ++++ & - & ++ \\
\hline IL-12-deficient & ++ & - & ++++ & + & + \\
\hline TNF- $\alpha$-deficient & $+/-$ & ++ & +++ & ++ & - \\
\hline $\begin{array}{l}\text { IFN- }- \text { /TNF- } \alpha \\
\text { deficient }\end{array}$ & $++++{ }^{c}$ & - & +++ & - & - \\
\hline
\end{tabular}

WT, wild type mice; ${ }^{\text {a }}$ Data were compiled from multiple experiments. A semiquantitative system scoring was used: ++++ , more than 10 -fold greater than levels detected in WT mice; +++, 2- to 10-fold greater than levels detected in WT mice; ++, equivalent to levels detected in WT mice; + , below levels detected in WT mice but above the assay's detection limit; -, not detected. ${ }^{\mathrm{b}}$ Hemorrhage denotes evidence for both hematocrit reductions (i.e. anemia) and positive tests for fecal occult blood (i.e. bleeding). (It was demonstrated that $T$. gondii-infected mice exhibited anemia due to a decrease in both survival time of red blood cells in the circulation and erythropoiesis $\left.(p<0.02)^{[423]}\right)$. ${ }^{c}$ Fibrin levels in IFN- $\gamma /$ TNF- $\alpha$ deficient mice were greatly elevated in comparison with WT mice yet significantly lower than those observed in IFN- $\gamma$-deficient animals.

because the stable complex formation rate of this inhibitor with tissue plasminogen activator (t-PA), urokinase-type plasminogenactivator (u-PA), and two-chain t-PA is described as $\mathrm{K}=10^{7} \mathrm{M}^{-1} \mathrm{~s}^{-}$ ', which for PAI-1 is a few orders of magnitude higher than that for other inhibitors, such as PAI-2, protease nexin, $\alpha 2$-macroglobulin, $\mathrm{C}^{\prime} 1$-esterase, and $\alpha 2$-antiplasmin $\left(\mathrm{K}=10^{0}-10^{5} \mathrm{M}^{-1} \mathrm{~s}^{-1}\right)$. Moreover, the plasma t-PA concentration is $70 \mathrm{pmol} / \mathrm{L}$ and its biological half-life is a few minutes, whereas the respective values for PAI- 1 are $1 \mathrm{nmol} / \mathrm{L}$ and 2 to $3 \mathrm{hrs}$ (in vitro, $\left.37^{\circ} \mathrm{C}\right)^{[463-465]}$.

Mullarky et a ${ }^{[434]}$ demonstrated an important role for the signal transducer and activator of transducer-1 (STAT1) in the suppression of infection-stimulated fibrin deposition via a STAT1-dependent pathway operating in endothelial cells, and interestingly, expression of PAI-1 also is STAT1 regulated ${ }^{[466]}$. IFN- $\gamma$ suppresses expression of PAI-1 in endothelial cells ${ }^{[467]}$, and as PAI-1 antagonizes fibrin degradation, thereby promoting fibrin deposition, STAT1-mediated suppression of PAI-1 expression in endothelial cells. This may well account for the effects of IFN- $\gamma$ on fibrin deposition during infection $^{[434]}$. In addition, IL-4 (an antiinflammatory cytokine) suppresses PAI-2 formation in stimulated human monocytes ${ }^{[427]}$.

\section{Transforming growth factor- $\beta$ (TGF- $\beta$ )}

This interleukin belongs to the family of related cytokines that influence inflammatory processes, cell growth, differentiation, apoptosis, and gene expression ${ }^{[123,468-470]}$. TGF- $\beta$ can upregulate the expression of integrin matrix receptors and inhibitors of matrixdegrading enzymes, such as PAI-1 ${ }^{[123,468-470]}$, which plays an important role in blood coagulation and fibrinolysis. TGF- $\beta 1$-induced PAI1 expression was found also in $\mathrm{VSMCs}^{[471]}$, and both these major causative biofactors are important in the pathophysiology of vascular disease and tissue fibrosis. This cytokine has profibrotic properties because it induces the expression of many matrix proteins including collagens, fibronectin, tenascin, and thrombospondin, and the net effect is the increased synthesis and assembly of matrix that result in fibrotic changes in affected tissues ${ }^{[123]}$. Activation of TGF- $\beta$ also involves generation of ROS and RNI that participate in development of atherosclerosis ${ }^{[123,472]}$.

\section{Nitric oxide (NO)}

Vascular endothelium-derived NO, a major mediator of endotheliumdependent vasodilation, has important antiinflammatory and antithrombotic properties, such as inhibiting leukocyte adhesion, 
limiting platelet adhesion and aggregation, and the expression of PAI$1^{[473-475]}$.

\section{Factor XIII (FXIII)}

Catani et $a l^{[476]}$ demonstrated that blood coagulation FXIII (fibrin stabilizing factor, plasma $t_{1 / 2}=3-5$ days) was a target for NO both in vitro and in vivo. NO donors inhibited FXIII activity in a dosedependent manner and inhibition occurred by S-nitrosylation of a highly reactive cystein residue. NO exerts its biological effect by interacting with a variety of molecular targets, mainly using metals such as iron, tiols and oxygen as reactive groups. NO has also been shown to interact with heme- and non-heme iron-containing proteins, thus regulating their activities. Nitrosonium ion $\left(\mathrm{NO}^{+}\right)$can react with protein thiol groups causing S-nitrosylation, thus affecting the function of these proteins. Interestingly, hydrogen sulfide $\left(\mathrm{H}_{2} \mathrm{~S}\right)$, an important gaseous signaling molecule that serves many important regulatory roles in pathophysiological states through NO, inhibited atherosclerosis through upregulating protein S-nitrosylation level in aorta $\mathrm{VSMCS}^{[477]}$. $\mathrm{H}_{2} \mathrm{~S}$ significantly reduced the aortic atherosclerotic lesion area and inhibited lipid and macrophage accumulation and vascular smooth muscle cell proliferation in apo $\mathrm{E}^{-/}$mice $\mathrm{e}^{[477]}$.

\section{von Willebrand factor (vWF)}

Elevated vWF levels are strongly associated with an increased risk of ischemic cardiovascular events. vWF probably participate in the process of atherogenesis, because results from animals studies suggested that at arterial branch point predilection sites, vWF deficiency or blockage had a protective effect against atherosclerosis ${ }^{[478]}$. Endothelial secretion of vWF from intracellular organelles is required for platelet adhesion to the injured vessel wall, because vWF binds platelets with collagen fibers in subendothelial layer and serves as a basis of platelets adhesion and aggregation. Torisu et $a l^{[479]}$ showed that autophagy regulated endothelial cell processing, maturation and secretion of vWF (autophagy is a reparative process by which cytoplasmic components are sequestrated in double-membrane vesicles and degraded on fusion with lysosomal compartments).

\section{Effect of blood flow rate}

Since the blood flow rate is the main determinant of in vivo plateletvessel wall interaction ${ }^{[480]}$, the platelets would tend to accumulate at the points of irregularities in blood flow dynamics, and might then release vasoactive agent(s) (possibly serotonin) and PAI- ${ }^{[481]}$, which is also liberated from endothelial cells of vessel walls during venous stasis $^{[482]}$. It should be noted that the platelet pool of PAI-1 is four- to six-fold greater than that of plasma ${ }^{[481,483]}$.

\section{Serotonin (5-HT)}

5 -HT, a potent vasoconstrictor in the large cerebral arteries, is considered to play a key role in atherosclerosis and to be implicated in ischemic cerebrovascular events followed by delayed neuronal death ${ }^{[484]}$. 5-HT released from activated platelets, not only accelarated aggregation of platelets but also promoted mitosis, migration, and contraction of VSMCs, and these effects contributed to thrombus formation and atherosclerosis ${ }^{[484]}$. Serotonin stimulated significantly the expression of PAI-1 and tissue factor mRNA in a concentrationand time-dependent manner through $5-\mathrm{HT}_{2 \mathrm{~A}}$ receptors, thus increasing procoagulant activity and reducing fibrinolytic activities of endothelial cells ${ }^{[485]}$. 5-HT-stimulated endothelial cells secreted a T lymphocyte-specific chemotactic cytokine with competence growth factor activity ${ }^{[486]}$. 5-HT alone and combined with thromboxane $\mathrm{A}_{2}$, potently induced vascular smooth muscle cell proliferation ${ }^{[487,488]}$. However, there is still controversy regarding the effects of 5-HT on endothelial cell proliferation ${ }^{[489]}$. Finally, Suguro et al ${ }^{[490]}$ found that 5-HT increased ACAT-1 activity in a concentration-dependent manner in primary monocyte culture, and suggested that 5-HT may play an important role in macrophage-derived foam cell formation. It must be noted that hyperserotoninemia has been reported in autistic and mentally retarded children ${ }^{[491,492]}$, as well as in the first degree relatives of these children ${ }^{[493,494]}$, and notably, Prandota et al ${ }^{[495]}$ found a significant association between autism and T. gondii infection $\left(\chi^{2}=\right.$ $8.11, p<0.0043 ; \mathrm{OR}=7.2 ; 95 \%$ CI: $1.56-33.7$ ).

\section{Glycated LDL}

Artwohl et $a l^{[496]}$ found that diabetic LDL triggered apoptosis in vascular endothelial cells. It was reported that glycated LDL increased adhesion molecule expression ${ }^{[497]}$ and modulated the fibrinolytic potential of vascular endothelial cells ${ }^{[498]}$. Furthermore, both in vitro- and in vivo-glycated LDL altered vasoactive response by attenuating NO synthesis ${ }^{[499]}$. The toxicity of glycated LDL and its role in the pathogenesis of atherosclerosis appeared to be related to its prolonged presence in the circulation and impaired cellular uptake ${ }^{[498,500]}$. It should be noted that $T$. gondii infection acquired prenatally and/or after birth may be responsible for development of both type 1 and 2 diabetes mellitus ${ }^{[501]}$.

\section{Magnesium deficiency}

Maier et al ${ }^{[502]}$ demonstrated that low magnesium concentrations reversibly inhibited endothelial cell proliferation, probably due to an up-regulation of IL-1 synthesis, a proinflammatory cytokine and potent inhibitor of endothelial cell growth. They observed also the up-regulation of VCAM-1 and PAI-1 levels after Mg deficiency.

All together, overproduction of proinflammatory cytokines, such as IFN- $\gamma$, TNF- $\alpha$, and NO, during latent chronic $T$. gondii infection may lead to serious physiopathological disturbances in the host, including anemia, hemorrhage, and blood coagulation disorders, i.e. the abnormalities that also play an important role in the clinical course of atherosclerosis.

IMPORTANT ROLE OF IRON IN DEVELOP-
MENT OF ATHEROSCLEROSIS AND NEURO-
DEGENERATIVE DISEASES

\section{Atherosclerosis}

According to the "iron hypothesis", iron overload is believed to be detrimental for the cardiovascular system, thus promoting atherosclerosis development and progression ${ }^{[503,504]}$. Excess of iron generates oxidative stress that hallmarks neurodegenerative diseases and atherosclerosis, a frequent disorder of aging ${ }^{[05]}$. Patients with Alzheimer's disease and Parkinson's disease showed a dramatic increases in their brain iron content which may contribute to the pathogenesis of these disorders, but so far the reason for iron accumulation is unknown ${ }^{[506]}$.

Iron accumulates in human atherosclerotic lesions ${ }^{[507]}$, and can enter into the lesion in the form of free hemoglobin $(\mathrm{Hb})$ that is released upon intravascular hemolysis or intraplaque hemorhage ${ }^{[508]}$. Cell-free $\mathrm{Hb}$ when oxidized releases heme and induces oxidative modification of $\operatorname{LDL}^{[509]}$. Iron mediates the oxidative modification of LDL, an important contributing factor to the pathogenesis of atherosclerosis ${ }^{[510]}$. Cholesterol levels in atherosclerotic lesions correlated with iron deposits ${ }^{[511]}$, and within the plaque, iron deposition and ferritin induction have been observed in endothelial 
cells and macrophages in early human lesions, and additionally in VSMCs in late lesions ${ }^{[503]}$. Interestingly, in hypercholesterolemic pigs, Gerrity ${ }^{[512]}$ found that monocytes were adherent to the lesion areas of aortic endothelium, in endothelial junctions, and in the intima. Intimal monocytes and altered intimal monocytes with enlarged cytoplasm and containing few lipid droplets were found to be phagocytic by their uptake of ferritin, which had penetrated the intima after intravenous injection. Circulating monocytes and those adherent to the endothelial surface did not contain ferritin in the animals ${ }^{[512]}$.

$\mathrm{Hb}$ derived iron is an important factor in determining macrophage differentiation and function in areas of intraplaque hemorrhage within human atherosclerosis. Habib et al ${ }^{[160]}$ showed that macrophages in areas of intraplaque hemorrhage demonstrated reduced intracellular iron and ROS which triggers production of antiinflammatory cytokines as well as genes involved in cholesterol efflux. Iron is a powerful catalyst in the generation of toxic free hydroxyl radicals through the oxidation of its ferrous $\left(\mathrm{Fe}^{2+}\right)$ (very toxic) to ferric $\left(\mathrm{Fe}^{3+}\right)$ via the Fenton reaction $\left(\mathrm{Fe}^{2+}+\mathrm{H}_{2} \mathrm{O}_{2} \longrightarrow \mathrm{Fe}^{3+} \mathrm{OH}^{-}+\mathrm{OH}\right)^{[513]}$, which are deleterious to living cells and most macromolecules ${ }^{[514]}$. Hydroxyl radicals can depolymerize polysaccharides, cause DNA breaks, inactivate enzymes, and initiate lipid peroxidation ${ }^{[160,515-517]}$. Extracellular ferric anion is bound to human serum lactoferrin (Lf) and transferrin, and transferrin is the iron transporter that allows cellular iron uptake ${ }^{[518]}$. Lf and transferrin maintain $\mathrm{Fe}^{3+}$ in a soluble and stable oxidation state, avoiding the generation of toxic free hydroxyl radicals ${ }^{[514]}$.

Hemolysis is often a result between the interaction of erythrocytes and mature atheromas resulting in the transition of ferrous to ferric forms of $\mathrm{Hb}$ which additionally leads to lipid peroxidation ${ }^{[519]}$, triggering endothelial activation, smooth muscle cell proliferation and macrophage activation ${ }^{[503]}$. Oxidized form $\mathrm{Hb}$ can also act as a proinflammatory agonist that targets vascular endothelial cells ${ }^{[20]}$.

Hydrogen peroxide $\left(\mathrm{H}_{2} \mathrm{O}_{2}\right)$ generated by mitochondria and other organelles permates in the lumen of secondary lysosomes which contain iron derived from cellular structures undergoing autophagic degradation ${ }^{[521,522]}$. The interaction between reactive ferrous iron and $\mathrm{H}_{2} \mathrm{O}_{2}$ resulted in the generation of hydroxyl radicals inducing lipid peroxidation and ceroid formation ${ }^{[523]}$. Lee et a ${ }^{[524]}$ reported that iron and ceroid deposits colocalized either extracellularly or intracellularly in foam cell-like macrophages or SMCs of advanced atherosclerotic plaques. Many cells in advanced human plaques contained a large number of ceroid-containing lysosomes, and impaired autophagy stimulates further accumulation of damaged mitochondria, increased ROS generation, additional accumulation of iron and enhanced nondegradable oxidation products such as ceroid ${ }^{[521]}$

\section{T. gondii infection}

The parasite must acquire nutrients from its host to survive and ensure its proliferation ${ }^{[525]}$. T. gondii is an auxotroph for iron ${ }^{[26,527]}$, cholesterol $^{[149,528]}$, tryptophan ${ }^{[529]}$, arginine ${ }^{[526,530]}$, polyamines ${ }^{[531]}$, purines $^{[332]}$, and other essential nutrients ${ }^{[146]}$. Parasites have an ability to acquire growth-essential iron from their hosts ${ }^{[533]}$. Possible sources of intracellular host iron include heme, iron released from transferrin at $\mathrm{pH}$ 5.5-6, and ferritin ${ }^{[533]}$. The metal is needed for catalysis of DNA synthesis and for a variety of enzymes concerned with electron transport and energy metabolism. Iron may also focus influx of neutrophils to sites of infection by generating oxidant-sensitive mediators ${ }^{[534]}$.

Dimier and Bout ${ }^{[527]}$ demonstrated that $T$. gondii invaded and proliferated in cultured primary rat enterocytes. Intestinal epithelial cells represent a rapidly renewing cell population, and their cell turnover has a mean duration time of 2-3 days in vivo and a few hours in vitro ${ }^{[335]}$. Experiments that used $\mathrm{Fe}^{2+}$ salt as well as carrier and chelator suggested that IFN- $\gamma$-treated enterocytes inhibited $T$. gondii replication within these cells by limiting the availability of intracellular iron to the parasite ${ }^{[527,536]}$. Data from an in vitro model of human monocytes infected with other pathogens, such as Legionella pneumophila $^{[537]}$, Histoplasma capsulatum ${ }^{[538]}$, or Trypanosoma cruzi $^{[539]}$ also have shown that limiting the availability of iron may represent a broadly effective intracellular antimicrobial mechanism. IFN- $\gamma$ inhibited the replication of T. gondii also in endothelial cells ${ }^{[56]}$, fibroblasts ${ }^{[540]}$, and retinal pigment epithelial cells ${ }^{[57]}$. In fibroblasts and retinal pigment epithelial cells, the mechanism of inhibition was via tryptophan starvation, while in astrocytes, the mechanism of IFN- $\gamma$ inhibition was independent of iron deprivation and reactive oxygen intermediates ${ }^{[541]}$.

Several pivotal proinflammatory cytokines such as IFN- $\gamma$, TNF- $\alpha$, IL-1, and IL-6 (these molecules are profusely generated during chronic $T$. gondii infection) participated in iron homeostasis, and the sequestration of this metal, mediated by these biomediators, was considered protective ${ }^{[542]}$. Proinflammatory cytokines and the acute phase protein hepcidin affected iron homeostasis leading to the accumulation of the metal ion in macrophages and subsequently to anemia of inflammation ${ }^{[543]}$. Hepcidin inhibited iron efflux through binding to and induction of the degradation of ferroportin1, a multipass protein found in the basolateral membrane of enterocytes ${ }^{[544]}$. Iron and the hormone erythropoietin modified innate immune responses by influencing IFN- $\gamma$-mediated (iron) or NF- $\mathrm{kB}$ inducible (erythropoietin) immune effector pathways in macrophages. Thus, the macrophages loaded with iron lost their ability to kill intracellular pathogens via IFN- $\gamma$-mediated effector pathways, such as NO generation ${ }^{[543]}$. On the other hand, NO directly controlled intracellular iron metabolism by activating iron regulatory protein, a cytoplasmic protein that regulates ferritin translation ${ }^{[543]}$. It was reported that NO mediated non-heme iron release from ferritin ${ }^{[545]}$, and ferritin, a hepatic protein and cytoprotective antioxidant of endothelium, may counteract some redox activity via ferroperoxidase in the vasculature ${ }^{[546]}$. Iron also regulated NOS activity by controling nuclear transcription of cytokine-inducible NOS mRNA ${ }^{[541]}$. Weiss et $a l^{[547]}$ showed that increased intracellular iron levels led to a decrease of NOS activity, whereas depletion of intracellular iron strongly enhanced the enzyme activity in IFN- $\gamma /$ LPS-stimulated macrophages, i.e. alterations (increases as well as decreases) in cellular iron availability caused regulation of NOS transcription in J774 cells ${ }^{[539]}$.

Macrophages and other host cells activated by IFN- $\gamma$ can be induced to form a flavoprotein that converts L-arginine to $\mathrm{NO}+$ L-citrulline ${ }^{[548]}$. NO causes efflux of non-heme iron from infected host cells. In the absence of L-arginine, IFN- $\gamma$-induced infected cells can lower their net uptake of iron. Cellular depletion of the metal via either mechanism suppresses DNA synthesis as well as the functioning of aerobic respiratory enzymes. Macrophage regulation of growth of other host cells during embryogenesis, immune responses, or immunosurveillance might involve iron depletion ${ }^{[548]}$.

Novel atherosclerotic markers indicating neutrophil activation represent Lf, an innate immune system protein with antiinflammatory and antioxidant properties ${ }^{[549,550]}$. Immunohistochemical staining of $\mathrm{Lf}$ was found in advanced human atherosclerotic carotid artery, as well as its co-localization with neutrophils and T and B lymphocytes in aortic plaques ${ }^{[51]}$. Lf appeared to be one of nine genes specifically induced predominantly in atherosclerotic aortic plaques ${ }^{[551]}$. Lf, a natural defense iron-binding protein is produced and secreted by glandular epithelial cells and circulating polymophonuclear neutrophils, and 
found mainly in milk, saliva, mucosal and other secretory fluids, has been found to possess antiinflammatory, antibacterial, antiparasitic, antimycotic, antiviral, and antineoplastic activity ${ }^{[52,553]}$. A principal function of $\mathrm{Lf}$ is that of scavenging free iron in fluids and inflamed areas so as to suppress free radical-mediated damage and decrease the availability of the metal to invading microbial and neoplastic cells. Lf normally maintains an iron saturation value of $25-35 \%$, and at values above $30 \%$, transferrin (present mainly in serum and lymph) begins to lose its effectiveness as a scavenger of hazardous iron ${ }^{[552,554]}$. Lf, a 78-kDa glycoprotein of the transferrin family, is structurally similar to transferrin with about $44 \%$ homology, and similar to transferrin Lf can reversibly bind two atoms of iron $\left(\mathrm{Fe}^{3+}\right)$. T. gondii utilize iron bound to inorganic or organic chelates, such as serum $\mathrm{Lf}$ and transferrin and/or intracellular iron compounds $\mathrm{Hb}$, hemin, ferritin and others ${ }^{[55,556]}$. Iron uptake by the parasite was limited by IFN- $\gamma$ through an unknown mechanism ${ }^{[518]}$. Dziadek et al ${ }^{[557]}$ found in human CaCo-2 epithelial cells, and mouse L929 fibroblasts, that extracellular $T$. gondii tachyzoites of BK strain (genotype I) bind specifically human serum Lf but not transferrin, and the binding was concentration-dependent. Moreover, the multiplication of the parasite was inhibited by human Lf high concentrations ( $\geqslant 100 \mathrm{mg} /$ $\mathrm{mL}$ ) in both cell lines ${ }^{[553]}$. Virtually all cells express a transferrin receptor on their surface, and the quantity of receptor molecules reflects the cellular iron requirements ${ }^{[518]}$. It should be noted that host cell expression of transferrin receptor was upregulated in $T$. gondii infected cells, suggesting that the parasite utilized the host endocytic pathway for iron acquisition ${ }^{[518,526]}$. Since the receptors for human Lf also are expressed on many human cells, they could provide a mechanism for the interaction between Lf binding T. gondii tachyzoites and host cells ${ }^{[558]}$. It appeared that the presence of iron was required for the Lf molecule to stimulate an increase of uptake and killing of an intracellular parasite by murine macrophages and human monocytes ${ }^{[59,560]}$, especially that $T$. gondii binds LF and not transferrin ${ }^{[577]}$. T. gondii membrane proteins ROP4 and ROP2 were found to be involved in human Lf acquisition ${ }^{[561]}$. Human Lf displayed cytoprotective (in a concentration-dependent manner) and antioxidant effects against $\mathrm{H}_{2} \mathrm{O}_{2}$-induced oxidative stress in human umbilical vein endothelial cells ${ }^{[562]}$. Immunomodulatory effects of Lf include interacting with monocytes and macrophages and modulating their function during inflammatory and infectious processes, e.g., increasing cytotoxic activity, cytokine production $\left(\mathrm{T}_{\mathrm{H}} 1\right)$ and expression of surface molecules ${ }^{[563]}$, enhancing phagocytosis, induction of apoptosis, and promoting $\mathrm{T}_{\mathrm{H}} 2$ cell responses in intestinal $\operatorname{mucosa}^{[564]}$. Moreover, Lf displayed beneficial effects on plasma lipid levels, because circulating Lf levels were inversely associated with fasting triglycerides $(p=0.001)^{[565]}$, and Lf administration led to decreased plasma triacylglycerol and free fatty acids levels, and to increased plasma HDL-cholesterol concentration accompanied by decreased hepatic cholesterol and triacylglycerol contents in rodents ${ }^{[566]}$.

In summary, iron accumulates in human atherosclerotic lesions and play an important role in the pathogenesis of these disorders because this metal may focus influx of neutrophils to sites of infection by generating oxidant-sensitive mediators. T. gondii is an auxotroph for iron and other nutrients. Experimental data suggested that IFN$\gamma$-treated enterocytes inhibited T. gondii replication within these cells by limiting the availability of intracellular iron to the parasite. Proinflammatory cytokines and the acute phase protein hepcidin affected iron homeostasis leading to the accumulation of the metal ion in macrophages and subsequently to anemia of inflammation. Iron modified innate immune responses by influencing IFN- $\gamma$-mediated immune effector pathways in macrophages. The macrophages loaded with iron lost their ability to kill intracellular pathogens via IFN- $\gamma$ mediated effector pathways, such as NO generation. NO mediated non-heme iron release from ferritin, and ferritin may counteract some redox activity in the vasculature. Extracellular $T$. gondii tachyzoites bound specifically human serum Lf, and the parasite membrane proteins ROP4 and ROP2 were involved in Lf acquisition. It must be noted that Lf is one of 9 genes specifically induced predominantly in atherosclerotic aortic plaques, and its principal function was that of scavenging free iron in fluids and inflamed areas and decrease the availability of the metal to invading microbial cells. Thus, a marked increase of iron content in both atherosclerotic lesions and brain tissues in neurodegenerative diseases may reflect a defense of the host against infection with the parasite and contribute to the pathogenesis of these disorders, especially that iron regulates NOS activity by controling nuclear transcription.

POSSIBLE LINK BETWEEN ELEVATED PLAS-
MA LEVELS OF TGF- $\beta 1$ IN ATHEROSCLE-
ROSIS, AND T. gONdii INFECTION. DUAL
ROLE: TGF- $\beta$ INHIBITS INFLAMMATION BUT
INCREASES INTRACELLULAR PARASITE REP-
LICATION

\section{Atherosclerosis}

Inflammation play an important role in atherosclerosis ${ }^{[567]}$. In advanced atherosclerosis, the serum concentration of active TGF- $\beta$ was found to be severely depressed ${ }^{[568,569]}$. Blann et al ${ }^{[570]}$ demonstrated also a significantly increased serum levels of TGF- $\beta$ receptors in atherosclerotic patients with ischemic heart disease and peripheral vascular disease, which correlated with serum total cholesterol levels $(p<0.001)$. Several authors further established that this cytokine reduced atherosclerosis by dampening $\mathrm{T}$ cell activation $^{[571,572]}$, while disruption of TGF- $\beta$ signaling in T cells resulted in acceleration of this inflammatory process ${ }^{[571,573,574]}$.

TGF- $\beta$ belongs to the family of related cytokines that influence cell growth, differentiation, apoptosis, inflammatory processes, and gene expression ${ }^{[468-470]}$. This is a multipotent cytokine with antiinflammatory activities, such as inhibition of proliferation, maturation and/or activation of macrophages, lymphocytes and $\mathrm{NK}$ cells ${ }^{[575-578]}$. TGF- $\beta$ is an important regulator of inflammation, being proinflammatory at low concentrations and antiinflammatory at high concentrations ${ }^{[579]}$. Activation of TGF- $\beta$ involves reactive oxygen species ${ }^{[472]}$. This cytokine is secreted by a number of cells, including regulatory $\mathrm{T}$ cells, macrophages, endothelial cells, dendritic cells, platelets, and vascular smooth muscle cells ${ }^{[580,581]}$. Tonkin and Haskins ${ }^{[582]}$ demonstrated that regulatory $\mathrm{T}$ cells transfer caused a reduction in the number of effector $\mathrm{T}_{\mathrm{H}} 1 \mathrm{~T}$ cells and macrophages, and also inhibited effector $\mathrm{T}$ cell cytokine and chemokine production.

TGF- $\beta$ has profibrotic properties because it induces the expression of many matrix proteins including collagens, fibronectin, tenascin, and thrombospondin, and the net effect is the increased synthesis and assembly of matrix that result in fibrotic changes in affected tissues $^{[123]}$. TGF- $\beta$ system mediated diabetic renal hypertrophy and fibrosis build-up due to the extracellular matrix production ${ }^{[583,584]}$, the cytokine was found to induce vaginal tissue fibrosis in animal model ${ }^{[585]}$, and the beta cell hypertrophy, beta cell damage and fibrosis, with reduction in insulin secretion, was characteristic for patients with type 2 diabetes mellitus ${ }^{[501,586]}$. 
TGF- $\beta$ plays a critical role in the suppression of lymphocyte proliferation and differentiation therefore preventing hazardous autoimmune responses, and its immunosuppressive effects are mediated through the inhibition of TNF- $\alpha$ and IL- ${ }^{[587]}$ and blocking the induction of adhesion molecules like ICAM- 1 and VCAM- $1^{[588-}$ ${ }^{590]}$. Filisetti and Candolfi ${ }^{[373]}$ reported that TGF- $\beta$ is well known for its immunosuppressive action on leukocyte cell lines. This cytokine was found to be an antagonist of TNF $\alpha$, TNF- $\beta$, IFN- $\gamma$ and IL$2^{[591,592]}$. The antiinflammatory action of TGF- $\beta$ control development of immunopathological processes related to $\mathrm{T}_{\mathrm{H}} 1$ immune response in the brain ${ }^{[593]}$ and the intestines ${ }^{[594]}$.

TGF- $\beta$ can also upregulate the expression of integrin matrix receptors and inhibitors of matrix-degrading enzymes, such as PAI- ${ }^{[471]}$, which plays an important role in blood coagulation and fibrinolysis. TGF- $\beta 1$-induced PAI- 1 expression was found also in $\mathrm{VSMCS}^{[471]}$, and both these major causative biofactors are important in the pathophysiology of tissue fibrosis and vascular disease.

\section{T. gondii infection}

Host protection to $T$. gondii infection involves $\mathrm{T}_{\mathrm{H}} 1$ type immune response of inflammatory cells, lymphocytes and macrophages with enhanced production of IFN- $\gamma$, TNF- $\alpha$, and IL-1 $\beta^{[373,595]}$. Activation of macrophages plays an important role in the host resistance against intracellular pathogens. Langermans et al ${ }^{[592]}$ found that the IFN- $\gamma$ induced toxoplasmastatic activity of macrophages was inhibited by TGF- $\beta$ (mean fold increase $=6.3$ ), which was also found for the IFN$\gamma$-induced production of TNF- $\alpha$, RNI and $\mathrm{PGE}_{2}$ by macrophages. It appeared that inhibition of TNF- $\alpha$ production was a key factor in the TGF- $\beta$-induced suppression of macrophage activation with respect to toxoplasmastatic activity and RNI production ${ }^{[592]}$. Activated macrophages by IFN- $\gamma$ inhibit parasite replication through a number of potent microbicidal mechanisms such as oxidative ${ }^{[596]}$ and nonoxidative $^{[597]}$ mechanisms, as well as the induction by IFN- $\gamma$ of enzyme indoleamine 2,3-dioxygenase that degrades tryptophan, which is required for the parasite replication ${ }^{[598]}$. Barbosa et a ${ }^{[599]}$ showed that treatments with IL-10 or TGF- $\beta 1$ induced a considerable augmentation in both $T$. gondii intracellular replication and invasion into BeWo trophoblastic cells, in contrast with HeLa cells. BeWo cells were unable to control replication of the parasite even in the presence of exogenous IFN- $\gamma^{[600]}$. Nagineni et al $l^{[210]}$ reported that in human retinal pigment epithelial cultures TGF- $\beta$ also enhanced parasite replication. Soluble extracts of $T$. gondii stimulated secretion of both TGF- $\beta 1$ and TGF- $\beta 2$ significantly. T. gondii infection completely inhibited secretion of the active form of TGF- $\beta 2$. In addition, Malipiero et $a l^{[601]}$ found that endogenous TGF- $\beta$ suppressed host defense against pathogen infection also in the central nervous system.

TGF- $\beta$ is downregulating tissue inflammation and immunity in the gastrointestinal tract (intraepithelial lymphocytes via the secretion of TGF- $\beta$ are critical in preventing inflammation of the small intestine villi following parasite infection), the central nervous systems, and the eye, where latent $T$. gondii reside and often become reactivated ${ }^{[594,602,603]}$. TGF- $\beta$ was found to increase in vitro replication of $T$. gondii in retinal cells ${ }^{[604]}$. Elevated expression of TGF- $\beta$ in vitreous, retina and retinal pigment epithelium has been correlated closely with retinal fibrosis and choroidal neovascularization ${ }^{[210]}$. One cannot therefore exclude that the development of fibrotic changes in atherosclerotic lesions are associated with the increased levels of this cytokine due to T. gondii infection because TGF- $\beta$ belongs to the biomediators favouring growth of the parasite ${ }^{[605]}$, and at the same time mediates balance between inflammation and fibrosis during plaque formation ${ }^{[606]}$.

Activated macrophages control growth by NO production ${ }^{[607]}$.
However, T. gondii active invasion inhibits NO production, allowing parasite persistence. The mechanism used by $T$. gondii to inhibit NO production persisting in activated macrophages depends on phosphatidylserine exposure. TGF- $\beta 1$ led to iNOS degradation, actin filament (F-actin) depolymerization, and lack of NF- $\mathrm{KB}$ in the nucleus $^{[607]}$.

Taken together, it seems that the increased levels of plasma TGF- $\beta$ reported in patients with atherosclerosis exert both beneficial and harmful effects because although this cytokine inhibits inflammation and autoimmunity, at the same time increases $T$. gondii replication in the host cells with further development of unwanted pathophysiological consequences, including fibrotic changes in atherosclerotic lesions. Final clinical effect of TGF- $\beta$ depends mainly of its serum levels, being proinflammatory at low concentrations and antiinflammatory at high levels.

\section{MAST CELLS (MCS) PLAY AN IMPORTANT ROLE IN THE PATHOGENESIS OF ATHERO- SCLEROSIS, AND SIMULTANEOUSLY EXERT A BENEFICIAL EFFECT IN HOST DEFENSE AGAINST T. gondii INFECTION}

\section{Atherosclerosis}

MCs are important in the development of both innate and acquired immunity, inflammation, and atherosclerosis ${ }^{[08-613]}$. MCs, macrophages, and neutrophils release TNF- $\alpha$, IFN- $\gamma$, and IL- 6 that induce expression of adhesion molecules in vascular endothelium and recruit of leukocytes, which is essential to the pathogenesis of vascular inflammatory diseases ${ }^{[614]}$. MCs by virtue of mediator secretion and degranulation, can regulate the function of endothelial cells, T lymphocytes, macrophages, and $\mathrm{VSMCs}^{[613]}$. The cells produce a number of biomediators, including histamine, tryptase, chymase, cathepsin G, platelet activating factor, leukotrienes, thromboxanes, prostaglandins, matrix-degrading metalloproteinases (MMP-1, MMP-3), inflammatory cytokines (TNF- $\alpha$, IL-4, IL-5, IL-6, IL-13), and chemokines (MCP-1, IL-8, RANTES) $)^{[613]}$. MCs can assist in the recruitment of monocytes and lymphocytes into vascular tissue, thereby propagating the proinflammatory respons $\mathrm{e}^{[144]}$, and are present in atherosclerotic lesions and thrombotic sites ${ }^{[615,616]}$. MCs were found in close association with macrophages and extracellular lipids, and at the sites of foam cell formation in atherosclerotic lesions ${ }^{[616]}$. MCs facilitate foam cell formation by promoting the formation of LDL aggregates, native and oxLDL uptake by macrophages, and by interfering with macrophage cholesterol removal by $\mathrm{HDL}^{[15-622]}$. Moreover, the cells provide a general anticoagulant function through actions of tryptase (fibrinogenolysis, inactivation of high molecular weight kininogen), heparin proteoglycans (anticoagulant, inhibition of platelet aggregation), and therefore might slow thrombus formation at the sites of atheromatous plaque rupture ${ }^{[13,620]}$.

\section{T. gondii infection}

The parasite is a potent $T_{H} 1$ type cytokine inducer, and while these proinflammatory cytokines are required to survive infection, their overproduction may lead to pathology and death of the host. Effector cells against $T$. gondii include monocytes ${ }^{[617]}$, IFN- $\gamma$ activated macrophages ${ }^{[420,624]}$, neutrophils ${ }^{[623]}$, and platelets ${ }^{[417]}$. MCs also participate in host defense against the parasite. Table $\mathbf{2 3}$ presented effects of mast cells incubation with $T$. gondii tachyzoites and influence of two different selective leukotriene inhibitors (5-lipooxygenase inhibitor A-63162 and 5-lipoxygenase-activated 
protein inhibitor MK-886) on MC degranulation and T. gondii cytotoxicity ${ }^{[625]}$.

MCs cells play a crucial role in the development of a protective immune response during resistance against $T$. gondii oral infection because of their ability to release proinflammatory mediators ${ }^{[625-629]}$. The cells degranulate and release LTB4, which damage tachyzoites ${ }^{[625]}$. Earlier study showed that LTB4-induced $T$. gondii cytotoxicity was characterized by vesiculation of the surface membrane and loss of the cytoplasmic contents of the organisms ${ }^{[624]}$, and the same cytotoxic changes were observed in tachyzoites after incubation with $\mathrm{MCs}^{[625]}$. Although the parasite inhibited mast cell degranulation by suppressing the mobilization of intracellular $\mathrm{Ca}^{2+}$ mediated by C-phospholipase ${ }^{[630]}$, many biomediators, such as IL-6, can also be released from mast cells "selectively", without degranulation ${ }^{[631,632]}$. In addition, it appeared that reactive oxygen species were not implicated in the mast cell-mediated toxoplasmacidal activity ${ }^{[625]}$.

Taken together, MCs play an important role in both development of atherosclerosis and host defense against $T$. gondii infection.

\section{PLATELETS AND SEROTONIN (5-HT) EN- HANCE MACROPHAGE FOAM CELL CELL FORMATION AND ARE CYTOTOXIC TO T. gondii TACHYZOITES (IN THE ABSENCE OF ANTIBODY)}

\section{Atherosclerosis}

5-HT, a potent vasoconstrictor in the large cerebral arteries, is considered to play a key role in atherosclerosis and to be implicated in ischemic cerebrovascular events followed by delayed neuronal death ${ }^{[484]}$. Ban et al ${ }^{[633]}$ found that plasma 5-HT concentrations, serum levels of hepatocyte growth factor (HGF), LDL cholesterol, and hsCRP, carotid artery intima-media thickness (IMT) and plaque frequency were significantly greater in patients with vascular dementia than in controls. There was a significant positive correlation of max IMT with 5-HT or HGF levels, and the authors suggested that increased plasma 5-HT levels and carotid atherosclerotic plaques may be involved in the pathogenesis and progression of vascular dementia ${ }^{[633]}$.

Atherogenesis involves platelet activation with subsequent serotonin release. 5-HT stimulated monocyte adhesion ${ }^{[48,634]}$, and enhanced macrophage foam cell formation with increased uptake of oxLDL (protein concentration $10-75 \mathrm{mg} / \mathrm{L})^{[188,635]}$. Suguro et $a l^{[188]}$ found that during differentiation of cultured human monocyte into macrophages expression of ACAT1 protein increased in a time-dependent manner. Serotonin increased ACAT1 activity in a concentration-dependent manner also in primary monocyte culture. In addition, 5-HT may contribute to inflammatory activation of the vessels during atherogenesis because it increased synthesis of IL-6 in human VSMCs, and this cytokine is a key molecule in chronic inflammation and was implicated in the progression of atherosclerosis ${ }^{[636]}$.

5-HT released from activated platelets, not only accelerated aggregation of platelets but also promoted mitosis, migration, and contraction of VSMCs, and these effects contributed to thrombus formation and atherosclerosis ${ }^{[484]}$. Serotonin stimulated significantly the expression of PAI-1 and tissue factor mRNA in a concentrationand time-dependent manner through $5-\mathrm{HT}_{2 \mathrm{~A}}$ receptors, thus increasing procoagulant activity and reducing fibrinolytic activities of endothelial cells ${ }^{[465]}$. 5-HT-stimulated endothelial cells secreted a $\mathrm{T}$ lymphocyte-specific chemotactic cytokine with competence
Table 23 Ultrastructural morphometric changes of MCs after interaction with $T$. gondii tachyzoites and effects of the selective inhibitors on these alterations (acc. to Henderson et al $^{[625]}$; with own modifications).

\begin{tabular}{|l|c|c|}
\hline Reaction mixture & $\begin{array}{c}\text { Mast cell } \\
\text { degranulation, } \\
\text { degranulated cells/ } \\
\text { total cells (\%) }\end{array}$ & $\begin{array}{c}\text { T. gondii cytotoxic } \\
\text { changes, damaged } T . \\
\text { gondii/total } \\
\text { T. gondii }(\%)\end{array}$ \\
\hline Mast cells & $27 / 305(8.9)$ & - \\
\hline T. gondii & - & $42 / 580(7.2)$ \\
\hline Mast cells + T. gondii & $278 / 398(69.8)$ & $134 / 306(43.8)$ \\
\hline $\begin{array}{l}\text { Mast cells + T. gondii }+ \\
\text { A-63162 }\end{array}$ & $71 / 341(20.8)^{\text {a }}$ & $32 / 234(13.4)^{\text {a }}$ \\
\hline $\begin{array}{l}\text { Mast cells + T. gondii }+ \\
\text { MK-886 }\end{array}$ & $44 / 206(21.4)^{\text {a }}$ & $36 / 300(12.0)^{\text {a }}$ \\
\hline $\begin{array}{l}\text { Mast cells + T. gondii }+ \\
\text { indomethacin }\end{array}$ & $252 / 399(63.2)$ & $144 / 389(37.0)$ \\
\hline
\end{tabular}

Mast cells $\left(2 \times 10^{6}\right)$ and T. gondii RH strain $\left(1.2 \times 10^{7}\right)$ were incubated in absence or presence of A-63162 $\left(10^{-6} \mathrm{M}\right)$, MK-886 $\left(10^{-6} \mathrm{M}\right)$, or indomethacin $\left(10^{-5} \mathrm{M}\right)$ in $1 \mathrm{~mL}$ of Tyrode's buffer for $30 \mathrm{~min}$ at $37^{\circ} \mathrm{C}$. Percent of degranulated mast cells and damaged tachyzoites was determined by morphometry. Data are means of 2-3 experiments for each condition. ${ }^{a} p<$ 0.05 vs mast cells $+T$. gondii.

growth factor activity ${ }^{[466]}$. This biomediator, alone and combined with thromboxane $\mathrm{A}_{2}$, potently induced VSMC proliferation ${ }^{[467,468]}$, but there is still controversy regarding its effects on endothelial cell proliferation $^{[469]}$.

\section{T. gondii infection}

Platelets may play an important role in the host defense against T. gondii. Human platelets were found to be cytotoxic to the parasite tachyzoites ${ }^{[637]}$. Adherence of platelets to the pahogen and disruption of surface membranes and internal architecture were observed ultrastructurally. The interaction of $T$. gondii with platelets resulted in a marked increase in $\mathrm{TXB}_{2}$ generation compared with that by unstimulated platelets ${ }^{[631]}$. Moreover, Henderson et $a l^{[638]}$ demonstrated that human platelets incubated with $T$. gondii tachyzoites released oxygenated products of both arachidonic acid and linoleic acid, 13-hydroxyoctadecadienoic acid (13-HODE) $(87.7 \%)$ and 9 -HODE (12.3\%). Further study ${ }^{[639]}$ revealed that $13-$ HODE, a product of linoleic acid metabolism, at concentrations higher or equal to $10(-8) \mathrm{M}$ rapidly induced cytotoxic changes in the parasite, including leakage of cytoplasmic contents in the space between the inner and outer parasite bilayer membrane units, which was followed by intracellular vacuolation and loss of cytoplasmic contents. Interestingly, Shamseddin et al ${ }^{[640]}$ showed that conjugated linoleic acid, a natural component of milk and diary products, in concentrations 50-250 $\mu \mathrm{M}$ induced apoptosis in virulent $\mathrm{RH}$ and avirulent Teheran strains of $T$. gondii, in HeLa cells.

\section{T. gondii INFECTION INCREASES LEPTIN LEVELS WHICH PLAY AN IMPORTANT ROLE IN TRIGGERING INFLAMMATION AND PARTICIPATE IN DEVELOPMENT OF ATH- EROSCLEROSIS}

\section{Atherosclerosis}

Leptin, the adipocyte-secreted hormone, is mainly produced by white adipose tissue and circulates in the blood in levels proportional to the fat mass, and both central and peripheral administration of leptin inhibited apetite and adiposity ${ }^{[64,642]}$. This adipocytokine modulates immune responses ${ }^{[643]}$, metabolism and inflammation ${ }^{[644]}$, and is a potential linker between obesity and 
chronic inflammation ${ }^{[645]}$. Structurally, it resembles IL-2 in particular and is a crucial T-cell growth factor ${ }^{[646]}$, and a potent negative regulator of IL-2 induced NK cell cytotoxicity ${ }^{[64]}$. The levels of this biomediator were increased in infections and autoimmune disorders, or after exposure to inflammatory stimuli such as LPS, TNF- $\alpha$, and IL-1 ${ }^{[641,648]}$.

Leptin accelerated CEs accumulation in human monocyte-derived macrophages by increasing ACAT1 expression and cholesterol efflux from these cells ${ }^{[208]}$. In human atherosclerotic lesions, leptin mediated atherosclerosis in vitro and in vivo, and induced (at doses $10-40 \mathrm{ng} / \mathrm{mL}$ ) proliferation of the human umbilical vein endothelial cells and elevation of matrix metalloproteinases (MMPs) MMP2 and MMP-9 expression, in a dose-dependent manner ${ }^{[649]}$. Leptin caused also concentration- and time-dependent increases in IL-6 production $^{[390]}$, which is the predominant mediator of the acute phase response, an innate immune mechanism triggered by infection and inflammation ${ }^{[388,389]}$. IL-6 levels were elevated in chronic inflammatory conditions, such as rheumatoid arthritis ${ }^{[50,651]}$.

\section{T. gondii infection}

It was demonstrated that increased leptin levels correlated with enhanced concentrations of inflammatory markers in morbidly obese individuals ${ }^{[645]}$, and recently, Reeves et al ${ }^{[652]}$ reported a significant positive association between $T$ gondii seropositivity and obesity $(p=0.01)$. Individuals who were obese had a markedly higher $T$. gondii IgG titers compared to normal weight participants ${ }^{[652]}$. Also Baltaci and Mogulkoc ${ }^{[653]}$ found that rats infected with $T$. gondii had significantly increased plasma leptin levels four weeks after the intraperitoneal injection of live parasites as compared with control animals (Table 24).

It must be emphasized that in obese prepubertal children statistically significant positive correlations were found between leptin and IL-2, IL-1 $\beta$, IL- 6 or TNF- $\alpha$ serum concentrations ${ }^{[654]}$ (Table 25), and interestingly, psychotropic drugs that induce weight gain in psychiatric patients also clearly activated the TNF- $\alpha$ system $^{[655]}$.

Multiple cytokines and acute inflammation that raise leptin levels may have a potential role also in inflammatory anorexia ${ }^{[641]}$, especially that leptin treatment increased energy expenditure (oxygen consumption) in animals ${ }^{[656]}$. It should be noted that in mice with $T$. gondii infection the dual anorexia and hypermetabolic states were associated with the elevation of TNF- $\alpha$, IL-1 $\beta$, IL-2, IL4, IL-5, IL6 , IL-10, and IFN- $\gamma$ levels ${ }^{[657,658]}$, and it was reported that various cachectic-anorexic conditions resulted from interactions among cytokines and neurotransmitters as mediators of neurologic and neuropsychiatric manifestations of disease ${ }^{[659,660]}$.

In summary, T. gondii infection caused increased leptin levels, which were found to be correlated with the enhanced concentrations of inflammatory markers in morbidly obese individuals, and recently, a significant positive association between $T$ gondii seropositivity and obesity has been reported. Because latent chronic $T$. gondii infection has a high prevalence worldwide, and $23 \%$ of the European adult population are now obese, while approximately $57 \%$ of the world's adult population is projected to be obese or overweight by $2030^{[661]}$, atherosclerosis associated with inflammation and obesity represent a serious global health hazard.

IMPORTANT ROLE OF CYSTEINE CATHEP SINS (CAT) IN FOAM CELL FORMATION AND GENERATION OF AMYLOID IN ATH-
EROSCLEROTIC ARTERIES. INFECTION OF THE HOST VASCULAR ENDOTHELIAL CELLS BY T. gondii TACHYZOITES BECOMES AN ADDITIONAL SOURCE OF SIMILAR ENZYMES BECAUSE THE PARASITE EXPRESSES FIVE MEMBERS OF THE CATHEPSIN PROTEASES, SUCH AS ONE CATL-LIKE, ONE CATB-LIKE, AND THREE CATC-LIKE PROTEASES

\section{Atherosclerosis}

Cathepsins contribute to several pathophysiological processes, including antigen presentation in the immune system ${ }^{[662,663]}$, neuropeptide and hormone processing ${ }^{[664]}$, and collagen turnover in bone and cartilage ${ }^{[665]}$. These enzymes have strong elastolytic and collagenolytic properties that form a distinct subgroup of atherosclerosis-related proteases because they affect extracellular matrix degradation and modulate inflammation, immune responses, and cellular functions ${ }^{[666-670]}$. Increased expression and translocation of lysosomal cathepsins contribute to macrophage apoptosis in atherogenesis $^{[669]}$.

Cathepsins of the cysteine protease family, such as CatB, C, H, F, $\mathrm{K}, \mathrm{L}, \mathrm{O}, \mathrm{S}, \mathrm{V}, \mathrm{W}$, and $\mathrm{X} / \mathrm{Z}^{[671]}$ function in terminal protein degradation optimally whithin acidic lysosomes ${ }^{[672]}$, and play an important role in development of atherosclerosis-based cardiovascular diseases ${ }^{[673-678]}$. Cathepsins are expressed in macrophages, endothelial and vascular smooth muscle cells (VSMCs) of atherosclerotic lesions ${ }^{[677]}$. Studies on vascular cells have shown that atherosclerosis-associated proinflammatory cytokines enhanced cysteine cathepsin expression and activity. It was demonstrated that although VSMCs and macrophages physiologically express negligible levels of CatL, S, and $\mathrm{K}$, incubation of these cells with proinflammatory cytokines, such as TNF- $\alpha$, IL- $1 \beta$, and IFN- $\gamma$, significantly induced the expression and secretion of those cathepsins and their collagenolytic and elastolytic activities $^{[673,676,679]}$.

Table 24 Body weight and plasma leptin concentrations in rats intraperitoneally infected with T. gondii (acc. to Baltaci \& Mogulkoc[653], with own modification).

\begin{tabular}{|l|c|c|c|}
\hline Study group & $\begin{array}{c}\text { Body weight before } \\
\text { the study (g) }\end{array}$ & $\begin{array}{c}\text { Body weight after } \\
\text { four weeks (g) }\end{array}$ & $\begin{array}{c}\text { Plasma leptin } \\
\text { levels (ng/mL) }\end{array}$ \\
\hline $\begin{array}{l}\text { Control rats } \\
(n=10)\end{array}$ & $266.00 \pm 32.81$ & $270.50 \pm 33.70$ & $4.09 \pm 1.15$ \\
\hline $\begin{array}{l}\text { Infected } \\
\text { animals } \\
(n=10)\end{array}$ & $263.50 \pm 44.16$ & $269.50 \pm 42.78$ & $7.53 \pm 1.55^{\text {a }}$ \\
\hline
\end{tabular}

Results represent mean \pm SD. ${ }^{\text {a }}$ Statistically significant result $(p<0.01)$.

Table 25 Serum proinflammatory cytokines and leptin concentrations in prepubertal obese children compared with controls (acc. to Aygun et $a l^{[654]}$; with own modification).

\begin{tabular}{|l|c|c|c|}
\hline Parameter & Obese children & Controls & $P$ value \\
\hline Leptin $(\mathrm{ng} / \mathrm{mL})$ & $19.9 \pm 7.4$ & $7.9 \pm 5.1$ & $<0.001$ \\
\hline $\mathrm{IL}-1 \beta(\mathrm{pg} / \mathrm{mL})$ & $33 \pm 8.9$ & $3.6 \pm 1$ & $<0.001$ \\
\hline $\mathrm{IL}-2(\mathrm{U} / \mathrm{L})$ & $0.4 \pm 0.1$ & $0.9 \pm 0.1$ & $<0.01$ \\
\hline $\mathrm{IL}-6(\mathrm{pg} / \mathrm{mL})$ & $45.2 \pm 11.8$ & $13.1 \pm 3.9$ & $<0.001$ \\
\hline TNF- $\alpha(\mathrm{pg} / \mathrm{mL})$ & $9.2 \pm 2.3$ & $3.9 \pm 1$ & $<0.001$ \\
\hline E-selectine $(\mathrm{ng} / \mathrm{mL})$ & $78 \pm 38$ & $59 \pm 29$ & $<0.01$ \\
\hline hsCRP $(\mathrm{mg} / \mathrm{L})$ & $4.1 \pm 4.8$ & $0.9 \pm 1.5$ & $<0.001$ \\
\hline
\end{tabular}

Results are mean $\pm \mathrm{SD}$; CRP, C-reactive protein; hs, high-sensitivity. 
Monocyte/macrophages express CatL implicated in atherogenesis and associated arterial remodeling. Lipoprotein modification and uptake by atherosclerotic lesion cells, mainly macrophages and VSMCs, are important pathological steps in the formation of atherosclerotic plaques ${ }^{[669,673,675,677,680-683]}$. CatL ${ }^{[680,684,685]}$ and $\mathrm{CatB}^{[686,}$ ${ }^{687]}$ expression is enhanced in human coronary atherosclerotic lesions, carotid lesions, and in abdominal aortic aneurysms ${ }^{[676]}$. CatB, CatL and $\mathrm{CatD}$, were found in macrophage-derived foam cells in lipid-rich plaque areas ${ }^{[68,689]}$. Macrophages, VSMCs, and endothelial cells can mobilize cathepsins also extracellularly, for example, the CatB, L, S, and $\mathrm{K}$ participated in plaque extracellular proteolysis ${ }^{[690]}$.

The role of cysteine cathepsin proteases in lipid uptake, storage, and efflux has been partly elucidated ${ }^{[675,677]}$. Cathepsins have the capability to degrade LDL and reduce cholesterol efflux from macrophages, aggravating foam cell formation ${ }^{[67]}$. Several cathepsins have been implicated in apoB-100 proteolytic modification, which enhances extracellular LDL particle aggregation, lipid droplet formation, and LDL retention to arterial proteoglycans ${ }^{[683]}$. After taking up the lipoproteins and modified lipoproteins, macrophages and VSMCs become foam cells filled with lipid droplets. These lipids or modified lipoproteins affect cysteine cathepsin cellular expression and localization. Oxidized lipids translocate $\mathrm{Cat} L$ and $\mathrm{CatB}$ to the cytosol and nuclei, which is consistent with the findings that lysosomal CatL and CatB localize to the cytoplasm and nuclei of apoptotic macrophages/foam cells in human carotid atheroma ${ }^{[69,691]}$.

Macrophages exposed to oxLDL or $7 \beta$-hydroxycholesterol, expressed high levels of CatB and CatL because of lysosomal destabilization, in addition to forming foam cells ${ }^{[669]}$. The cathepsins translocated from lysosomes to cytosol or nuclei, caused foam cell apoptosis in the development and progression of atheroma ${ }^{[673]}$. In addition, lysosomal cell permeabilization due to exogenous reactive oxygen species may induce lysosomal leakage leading to release of cathepsins into the cytoplasm ${ }^{[675,692]}$. Cysteine cathepsins participated in the degradation of HDLs, thus reducing macrophage foam cell cholesterol efflux ${ }^{[675]}$

CatB has been implicated in the pathogenesis of inflammatory diseases and in many aspects of atherosclerosis ${ }^{[677,693]}$. CatB, CatC, and CatL showed increased activity in the human aneurysm wall and thrombus ${ }^{[694-696]}$, and CatB mRNA levels were increased in unstable regions of human plaque ${ }^{[687]}$. CatB cathepsin has been shown to activate IL- $1 \beta$ converting enzyme caspase- 1 , important for secretion of the mature IL-1 $\beta$ implicated in the pathogenesis of atherosclerosis $^{[663,697]}$

CatC was involved in immunological response because it activated granzymes $\mathrm{A}$ and $\mathrm{B}$, neutrophil elastase, and $\mathrm{CatG}^{[698]}$, and was found to be upregulated in $\mathrm{CD}^{+} \mathrm{T}$ cells in the thymus and spleen ${ }^{[699]}$. Interestingly, CatC can be activated by CatL or CatS, but not by autocatalytic processing ${ }^{[700]}$.

CatL is one of the most potent collagenases and elastases cleaving mature insoluble elastin ${ }^{[678]}$. This proatherogenic cathepsin is involved in death of macrophages, necrotic core formation and development of atherosclerotic plaque instability ${ }^{[701]}$. CatL is expressed in macrophages, VSMCs, and endothelial cells, and is located in the fibrous cap, tunica media, and macrophage-rich shoulder regions ${ }^{[676]}$. Macrophage apoptosis was found to be significantly correlated with expression of CatL in cell nuclei and membranes, and it was established that this cathepsin and TGF- $\beta$ were overexpressed in cells subjected to mitochondrial metabolic stress ${ }^{[702]}$.

CatL stimulated autophagy and inhibited apoptosis of oxLDLinduced endothelial cells ${ }^{[703]}$. Thus, the proatherogenic effect of CatL was partly neutralized by inducing autophagy and inhibiting apoptosis in early stages of atherosclerosis ${ }^{[703]}$. Wei et al ${ }^{[703]}$ showed that oxLDL increased CatL protein expression and activation, inducing endothelial cell autophagy and apoptosis, and increasing endothelial cell permeability. The enhanced permeability of the endothelium is the first event in the cascade of bioprocesses leading to atherosclerotic formation, which leads to lipid infiltration and accumulation within the arterial wall ${ }^{[703]}$. Inhibiting apoptosis lowered the permeability of monolayers to $\operatorname{LDL}^{[704]}$.

All together, cysteine protease cathepsins, such as for example CatB, CatC, and CatL, have been shown to play a critical role in immunity and development of lesions in atherosclerosis.

\section{T. gondii infection}

Cysteine proteinases play a major role in invasion and intracellular survival of a number of pathogenic parasites, including T. gondii ${ }^{[705]}$. Cathepsin proteases act clasically as lysosomal hydrolases that digest endogenous and exogenous endocytosed polypeptides ${ }^{[706]}$. They function in microneme and rhoptry protein maturation, host cell invasion, replication, and nutrient acquisition ${ }^{[707,708]}$. Que et al ${ }^{[705]}$ showed that the $\mathrm{TgCatB}$, toxopain-1, was localized to rhoptries (secretory organelles required for parasite invasion into cells), and was critical for parasite invasion and rhoptry protein processing. Toxopain-1 was also found to be critical for infection in a chicken model of congenital toxoplasmosis ${ }^{[709]}$.

The parasite has a limited number of cystein proteinases, with one cathepsin $\mathrm{B}(\mathrm{TgCPB})$, one cathepsin $\mathrm{L}(\mathrm{TgCPL})$ and three cathepsins C $(T g C P C 1,2,3)^{[710]}$. It was demonstrated that $T$. gondii tachyzoites ingested host cytosolic proteins and digested them using CatL and other proteases within its endolysosomal system ${ }^{[708]}$. Both virulent type I and avirulent type II strain parasites ingested and digested hostderived proteins, indicating that the pathway is not restricted only to highly virulent strains. Larson et al ${ }^{[711]}$ established that $\operatorname{TgCPL}$ was found in multiple puncts throughout the cytoplasm of intracellular replicating parasites, and was associated with a discrete vesicular structure in the apical region of extracellular parasites.

Cathepsins are critical to the parasite growth and differentiation ${ }^{[707]}$. T. gondii cathepsins were required for peptide degradation in the parasitophorous vacuole (PV), as the degradation of the marker protein, E. coli $\beta$-lactamase, secreted into the $\mathrm{PV}$ of transgenic tachyzoites was completely inhibited by the CatC inhibitor ${ }^{[707]}$. Tables 26 and 27 summarized classes and specific cathepsin-like proteases of $T$. gondii proteases, their function and localization, and the parasite stage expressions ${ }^{[708,712]}$. $T g C P C 1$ was found to be the most highly expressed cathepsin mRNA in tachyzoites, and three cathepsins, $T g \mathrm{CPB}, T g \mathrm{CPC} 1$ and $T g \mathrm{CPC} 2$, were undetectable in in vivo bradyzoites ${ }^{[708]}$.

Infection of the host vascular endothelial cells by $T$. gondii tachyzoites may be an important source of additional cathepsins activity, which will be superimposing on the host cell normal/ pathologic cathepsin activities. It should be also added that, for example, CatB suppresses proliferation of peripheral blood mononuclear cells ${ }^{[718]}$, and therefore participates in the immunosuppressive activity of the parasite proteases that can lead to impairment of the host immune defense. As a result, T. gondii infection may play a crucial role in triggering, maintaining and progression of atherosclerosis, especially because of the rapid proliferation of tachyzoites in endothelial cells ${ }^{[43]}$, and global burden of congenital and acquired toxoplasmosis ${ }^{[25,30]}$.

Finally, it must be noted that there was a prevalence and pathology of amyloid in atherosclerotic arteries ${ }^{[719]}$, and active forms of Cat B, CatL, and CatD, were found to be associated with amyloid deposits 
Table 26 Proteases characterized in T. gondii, their classes, function and localization (acc. to Li et al ${ }^{[712]}$; with own modification).

\begin{tabular}{|c|c|c|c|}
\hline Protease class & Specific protease & Protease function and localization & Refs \\
\hline \multirow{3}{*}{ Cysteine } & $\mathrm{TgCPB} /$ Toxopain-1 & Invasion; rhoptry protein processing & {$[705,709]$} \\
\hline & TgCPCs & Growth and replication; cleavage of dipeptides from $\mathrm{N}$-terminal in cytosol & {$[707]$} \\
\hline & $\operatorname{TgCPL}$ & Invasion; maturation of micronemal proteins; localized to the vacuolar compartment & [713] \\
\hline Metalloproteinase & Toxolysin 4 & Invasion/egress; localized to the micronemes & [714] \\
\hline \multirow{3}{*}{ Serine } & TgSUB1 & Invasion; maturation of rhoptry proteins; host cell attachment; localized to the micronemes & [715] \\
\hline & TgSUB2 & Invasion; maturation of rhoptry proteins; localized to the rhoptries & [716] \\
\hline & $\operatorname{TgROM} 2,4,5$ & Surface protein sheddase; localized to tachyzoite plasma membrane & [717] \\
\hline
\end{tabular}

Table 27 Features of $T$. gondii cathepsin-like proteases (acc. to Dou and Carruthers ${ }^{[708]}$; with own modification).

\begin{tabular}{|l|c|c|c|}
\hline $\begin{array}{l}\text { Cathepsin } \\
\text { protease }\end{array}$ & $\begin{array}{c}\text { Mature molecular } \\
\text { weight (kDa) }\end{array}$ & Activity & $\begin{array}{c}\text { T. gondii } \\
\text { stage expression }\end{array}$ \\
\hline $\mathrm{TgCPL}$ & 30 & endopeptidase & tachyzoite/bradyzoite \\
\hline $\mathrm{TgCPB}$ & 28 & $\begin{array}{c}\text { endo/ } \\
\text { exopeptidase }\end{array}$ & tachyzoite/bradyzoite \\
\hline $\mathrm{TgCPC} 1$ & 35 & exopeptidase & tachyzoite \\
\hline $\mathrm{TgCPC} 2$ & 44 & exopeptidase & tachyzoite \\
\hline $\mathrm{TgCPC} 3$ & 32 & exopeptidase & sporozoite \\
\hline
\end{tabular}

TgCPC1 was found to be the most highly expressed cathepsin mRNA in tachyzoites, and three cathepsins, TgCPB, TgCPC1 and TgCPC2, were undetectable in in vivo bradyzoites ${ }^{[708]}$.

in Alzheimer's disease $\mathrm{e}^{[720-722]}$. These findings are compatible with our earlier suggestions that latent chronic $T$. gondii infection may be at least in part responsible for the generation of amyloid plaques characteristic for the brains of patients with Alzheimer's disease and Down's syndrome ${ }^{[222,223]}$.

In summary, infection of host vascular endothelial cells by $T$. gondii tachyzoites may become an additional source of cysteine cathepsin activities that are superimposing on the activity of similar enzymes physiopathologically present in the host cells, because the parasite's tachyzoites, bradyzoites, and sporozoites express several members of these proteases.

\section{TREATMENT WITH GLUCOCORTICOIDS (GCS) ACCELERATED AND/OR EXACER- BATED DEVELOPMENT OF ATHEROSCLE- ROSIS AT LEAST IN PART BY ENHANCING PROLIFERATION OF T. gONdIi TACHYZOITES IN MACROPHAGES AND FOAM CELL FOR- MATION}

\section{Atherosclerosis}

Prolonged chronic stress associated with infection and inflammation can upregulate the levels of GCs and catecholamines leading to various diseases $^{[723]}$, and specifically cardiovascular diseases have been associated with elevated levels of IL- $1 \beta$, TNF- $\alpha$, IL- 6 , and IFN- $\gamma^{[724]}$. GCs have been reported to interfere with a variety of both intra- and extracellular activities of mononuclear phagocytes, and GC receptors have been identified in monocytes and macrophages ${ }^{[725]}$. Hydrocortisone and prednisone diminished human monocyte chemotaxis ${ }^{[726]}$, bactericidal capacity ${ }^{[727,728]}$, receptor activity for IgG and C3 complement ${ }^{[727]}$, and specifically inhibited macrophage elastase, collagenase, and PAI secretion ${ }^{[729]}$. Moreover, GCs inhibited the expression and action of most cytokines ${ }^{[730]}$. These drugs exerted an antiinflammatory effect by down regulating the production and secretion of proinflammatory cytokines such as TNF- $\alpha$, IL-1 $\beta$, IL-
2, IL-6, and the chemokines macrophage inhibitory protein (MIP)-1, and macrophage chemotactic protein/s (MCP)-1 and 2 from epithelial cells and macrophages, dendritic cells, $\mathrm{T}$ cells, and through apoptosis of $\mathrm{T}$ cells ${ }^{[731,732]}$. GCs not only down-regulated proinflammatory cytokines, they also generated a functionally active, antiinflammatory phenotype in human monocytes that suppressed inflammatory processes and, thus, induced resolution of inflammation ${ }^{[733]}$.

Many studies provided the experimental and clinical evidence that treatment with GCs accelerated and/or exacerbated development of atherosclerosis ${ }^{[194,734,735]}$. Accumulating evidence had demonstrated that foam cells were formed from macrophages in vitro at high concentrations $(80-160 \mu \mathrm{g} / \mathrm{mL})$ of oxLDL ${ }^{[736,737]}$. Moreover, several authors showed that dexamethasone (DXM, $1 \mu \mathrm{M}$ ) (a synthetic GC receptor agonist) could promote the macrophage-derived foam cell formation (a hallmark of early atherosclerotic lesions ${ }^{[738,739]}$ ) at lower concentration of ox-LDL in vitro, and that the drug increased the formation of CEs in macrophages and human SMCs in a dosedependent manner ${ }^{[201,740]}$. The finding that DXM could promote the foam cell formation at lower ox-LDL concentration might be partially caused by the up-regulation of ACAT1 gene expression at the transcriptional level and the enhancement of cholesterol esterification $^{[194]}$.

\section{T. gondii infection}

Wang et $a l^{[741]}$ showed that the growth rate of $T$. gondii RH strain tachyzoites was significantly increased in the peritoneal macrophages of rats treated with GCs in vivo compared with control cells (Table 28). They also demonstrated a significant inhibition of NO production in the macrophages collected from the rats receiving treatment with GCs compared with the control animals (Table 29). In addition, treatment with GCs inhibited the expression of iNOS mRNA in rat peritoneal macrophages ${ }^{[741]}$. [Nb. Costa et al ${ }^{[742]}$ showed that $T$. gondii infection slightly upregulated glucocorticoid-induced TNF receptor expression in Treg cells and B cells, but the most robust increment in expression was observed in macrophages and dendritic cells] These findings are consistent with the results obtained by Nishikawa et $a l^{[743]}$ that $T$. gondii decreased NO production in the peritoneal macrophages, and with the suggestion that the parasite could partially decrease NO production in the infected host cells and therefore escape the immune defense reaction in the host ${ }^{[744]}$.

Jones et $a l^{[745]}$ also found that DXM (the GC receptor ligand) significantly reduced LPS-induced macrophages production of NO. It was reported that DXM can inhibit iNOS expression in LPS-treated murine macrophages by destabilizing the mRNA transcript ${ }^{[746]}$. The $\mathrm{GC}$ receptor has also been demonstrated to interact with $\mathrm{NF}-\kappa \mathrm{B}$, which has a role in induction of iNOS, therefore providing another possible means of inhibiting NO production ${ }^{[747]}$. In addition, LPSinduced IL-12 generation also could be downregulated by the GC. Masur et $a l^{[748]}$ observed that even therapeutic concentrations of hydrocortisone rendered normal macrophages unresponsive to IFN- $\gamma$ 
and prevented the induction of the activated mononuclear phagocyte the capacity to respond to $T$. gondii ingestion with an enhanced oxidative respiratory burst, and the ability to inhibit intracellular parasite replication.

GCs are widely used for the treatment of autoimmune diseases and after bone marrow transplantation, sometimes resulting in development of acute toxoplasmosis in these patients ${ }^{[741,749,750]}$. Approximately one-third of transplant recipients experienced neuropsychiatric and other complications including encephalopathy, stroke, seizures, loss of vision, cardiovascular events, and de novo lymphoproliferative disorders (lymphoma, glioma) ${ }^{[751-754]}$. Organ transplant recipients had a three- to fourfold higher incidence of malignant diseases development compared with the general population $^{[752]}$, and many of CNS lymphomas were associated with prior Ebstein Barr virus infections ${ }^{[755]}$. Neurologic complications of liver transplantation were more common than that of other solid organ transplants (13-47\%) ${ }^{[753]}$. Recurrent ocular toxoplasmosis has also been reported in patients receiving systemic corticosteroid therapy (dose range, $0.27-1.23 \mathrm{mg} / \mathrm{kg} / \mathrm{day})^{[756]}$. The study of Ahlbom et $a l^{[757]}$ suggested that prenatal exposure to excess of GCs increased the susceptibility of cerebellar granule cells to oxidative stress-induced cell death, and elevated maternal cortisol levels during pregnancy have been associated with reduced childhood $\mathrm{IQ}^{[758]}$. It must be emphasized that in mice brains infected with $T$. gondii electron microscopy revealed that cortisone increased the amount of tachyzoites, cysts and cystozoites, as the rupture of cysts released a highly resistant antigen of cystozoite type ${ }^{[759]}$. All these CNS abnormalities, clinical complications, and developmental disturbances observed during therapeutic use of GCs could be due to the increased proliferation of $T$. gondii in the host cells induced by the increased levels of GCs and other immunosuppressive drugs that markedly affected physiologic balance between profusely generated type $\mathrm{T}_{\mathrm{H}} 1$ and type $\mathrm{T}_{\mathrm{H}} 2$ cytokines $^{[760,761]}$.

Taken together, several authors showed that DXM could promote the macrophage-derived foam cell formation at lower concentration of ox-LDL in vitro, and that the drug increased the formation of CEs in macrophages and human SMCs in a dose-dependent manner. These changes might be partially caused by the up-regulation of ACAT1 gene expression and thus the enhancement of cholesterol esterification. However, it should be noted that the growth rate of RH strain T. gondii tachyzoites was significantly increased in the peritoneal macrophages of rats treated with GCs in vivo compared with control cells. A significant inhibition of NO production in the macrophages collected from the rats receiving treatment with GCs was also observed. Moreover, GCs inhibited the expression and action of most cytokines involved in inflammation. Thus, all these molecular processes may at least in part explain an important role of GCs in development of atherosclerosis.

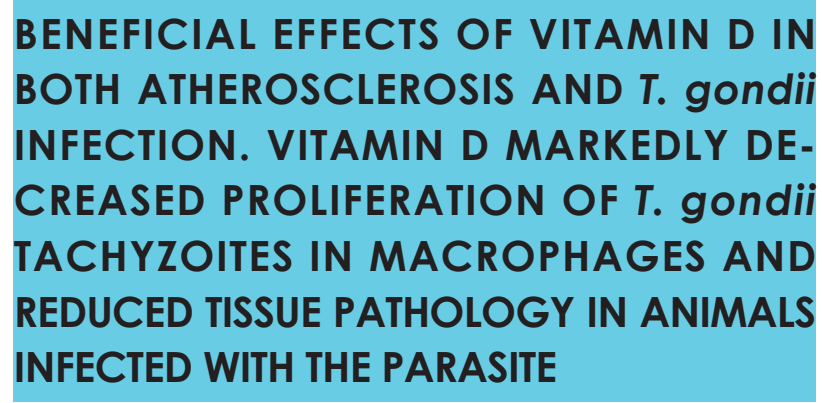

Atherosclerosis

Vitamin D regulates wide range of physiological and pathological
Table 28 Proliferation of $T$. gondii tachyzoites in peritoneal macrophages of rats treated with glucocorticoids (GCs) (acc. to Wang et al ${ }^{[21]}$; with own modification).

\begin{tabular}{|l|c|c|c|}
\hline \multirow{2}{*}{ GCs } & \multicolumn{3}{|c|}{ Number of $T$. gondii per $\mathbf{1 0 0}$ cells } \\
\cline { 2 - 4 } & $\mathbf{1 ~ h r}$ & $\mathbf{1 2} \mathbf{~ h r s}$ & $\mathbf{2 4} \mathbf{~ h r s}$ \\
\hline DXM & $35 \pm 3$ & $55 \pm 8^{\text {a }}$ & $176 \pm 15^{\text {a }}$ \\
\hline HSS & $39 \pm 5$ & $81 \pm 12^{\text {a }}$ & $227 \pm 14^{\text {a }}$ \\
\hline MP & $37 \pm 6$ & $85 \pm 14^{\text {a }}$ & $242 \pm 16^{\text {a }}$ \\
\hline Control & $39 \pm 5$ & $23 \pm 4$ & $16 \pm 4$ \\
\hline
\end{tabular}

Results are expressed as means \pm SD, number of rats $=3$. ${ }^{a} p<0.01 \mathrm{vs}$ control. Rats were injected intramuscularly with a dose of GCs for 7 days, macrophages were harvested and cultured for $12 \mathrm{hrs}$ and then incubated with $T$. gondii at the ratio 1:1 (parasites/macrophages $=1: 1$ ). DXM, dexamethasone; HSS, hydrocortisone sodium succinate; MP, methylprednisone.

Table 29 Nitrite production by peritoneal macrophages from rats treated with glucocorticoids (GCs) (acc. to Wang et al ${ }^{[21]}$; with own modification).

\begin{tabular}{|l|c|c|c|}
\hline \multirow{2}{*}{ GCs } & \multicolumn{3}{|c|}{ Nitrite production $(\boldsymbol{\mu M})$} \\
\cline { 2 - 4 } & $\mathbf{1 2} \mathbf{~ h r s}$ & $\mathbf{2 4} \mathbf{~ h r s}$ & $36 \mathbf{h r s}$ \\
\hline DXM & $9.78 \pm 0.37^{\mathrm{a}}$ & $14.31 \pm 1.22^{\mathrm{a}}$ & $17.99 \pm 1.08^{\mathrm{a}}$ \\
\hline HSS & $8.19 \pm 0.32^{\mathrm{a}}$ & $11.05 \pm 0.74^{\mathrm{a}}$ & $13.51 \pm 1.45^{\mathrm{a}}$ \\
\hline MP & $7.86 \pm 0.46^{\mathrm{a}}$ & $10.65 \pm 0.98^{\mathrm{a}}$ & $12.90 \pm 0.99^{\mathrm{a}}$ \\
\hline Control & $14.44 \pm 1.04$ & $25.41 \pm 1.44$ & $30.85 \pm 1.62$ \\
\hline
\end{tabular}

Results are expressed as means $\pm \mathrm{SD}$, number of rats $=3 .{ }^{\mathrm{a}} p<0.01 \mathrm{vs}$. control. DXM, dexamethasone; HSS, hydrocortisone sodium succinate; MP, methylprednisone. Peritoneal macrophages isolated from rats were treated with GCs for 7 days, and then incubated for 12, 24, and 36 hrs. $\mathrm{NO}_{2}{ }^{-}$production in the supernatant of the cell culture medium was measured by the Griess reaction.

processes characteristic for atherosclerosis, including vascular cell growth, migration, and differentiation, immune response modulation, cytokine expression, and inflammatory and fibrotic pathways ${ }^{[762]}$. Vitamin D deficiency is affecting more than one billion people to approximately $50 \%$ of population worldwide ${ }^{[762-764]}$. Shortage of vitamin D induced atherosclerosis and high blood pressure in mice ${ }^{[765]}$. Epidemiological and clinical evidence linked vitamin $\mathrm{D}$ deficiency to cardiovascular diseases in humans ${ }^{[766]}$, including carotid atherosclerosis ${ }^{[762,767,768]}$. Vitamin D receptor (VDR) is present in all cells implicated with atherosclerosis, such as endothelial cells, vascular smooth muscle cells, and immune cells ${ }^{[762]}$. It was demonstrated that vitamin D inhibited foam cell formation by suppressing oxidized and acetylated LDL-derived cholesterol uptake in macrophages from type 2 diabetes patients ${ }^{[769]}$, and decreased atherosclerosis by regulating $\mathrm{T}$ lymphocyte functions ${ }^{[770]}$. Vitamin D exerted also protective effects on endothelial cell dysfunction, inflammatory processes that precede atherosclerosis ${ }^{[771]}$, regulated the expression of profibrotic and antifibrotic factors ${ }^{[722]}$, and inhibited formation of atherosclerosis calcification ${ }^{[773,774]}$.

Vitamin D was found to modulate both the innate and acquired immune systems ${ }^{[775-778]}$. Macrophages, endothelial cells, and smooth muscle cells, among others, are able to transform $25(\mathrm{OH})_{3} \mathrm{D}_{3}$ to its active hormonal form, 1,25-dihydroxyvitamin D $\left(1,25(\mathrm{OH})_{2}\right.$ $\mathrm{D}_{3}$ ). The active metabolite primarily mediated its effects through the intracellular $\mathrm{VDR}^{[779]}$, which is expressed in most cell types of the immune system, in particular in antigen presenting cells such as monocytes, macrophages, and in dendritic cells ${ }^{[780]}, \mathrm{B}$ cells, as well as in $\mathrm{CD} 4^{+}$and $\mathrm{CD} 8^{+} \mathrm{T}$ cells $\mathrm{s}^{[775,778]}$. Vitamin D favors a mononuclear cell phenotype, increasing VDR expression on monocytes and macrophages ${ }^{[779]}$. Activation of VDR altered transcription, proliferation, and differentiation of immune cells ${ }^{[779]}$, and modulated immune responses both indirectly, by reducing the activation of proinflammatory $\mathrm{T}$ cells by antigen-presenting cells ${ }^{[781]}$, 
and directly, by inhibiting T and B cells proliferation ${ }^{[775,782]}$. Active vitamin $\mathrm{D}$ and corticosteroids exerted additive immunosuppressive effects on $\mathrm{T}_{\mathrm{H}} 1$ responses ${ }^{[783]}$. All these actions finally result in a $\mathrm{T}_{\mathrm{H}} 2$ type-driven antiinflammatory state in the host ${ }^{[784,785]}$. Table $\mathbf{3 0}$ listed multiple actions of vitamin D in the immune system. Several of the above-presented actions of vitamin D on immune system may have beneficial effects on clinical course and outcome of patients with atherosclerosis. It should be added that also vitamin A exerted favorable effects in patients with atherosclerosis because it decreased cytotoxicity of oxidized LDL and improved peripheral blood mononuclear cells viability ${ }^{[801]}$.

\section{T. gondii infection}

An infectious pathogenesis put forward that intracellular parasite infection disrupt the vitamin D regulated immune system resulting in persistent infection and chronic inflammation. Studies showed that $1,25(\mathrm{OH})_{2} \mathrm{D}_{3}$ directly increased antimicrobial peptide gene expression which could enhance host defense against infection ${ }^{[802,803]}$. Vitamin D was also required for IFN- $\gamma$-mediated antimicrobial activity of human macrophages ${ }^{[804]}$.

Vitamin D facilitate neutrophil motility and fagocytic function ${ }^{[05]}$. Circulating vitamin D levels have a direct influence on macrophages, increase their "oxidative burst" potential, such as for example maturation and generation of cytokines, hydrogen peroxide, and acid phosphatase ${ }^{[787,806]}$. Vitamin $1,25-\mathrm{D}_{3}$ inhibited proliferation of $\mathrm{T}_{\mathrm{H}} 1$ cells through impairing production of IL-2, TNF- $\alpha$, and IFN, as well as $T_{H} 17$ cells, and thus skewing cytokine production toward a $\mathrm{T}_{\mathrm{H}} 2$ phenotype ${ }^{[787,807]}$. Vitamin D may thus improve outcomes by reducing both local and systemic inflammatory actions as a result of modulating cytokine responses and decreasing TLR activation ${ }^{[08]}$.

Finally, it must emphasized that vitamin D significantly decreased in vitro proliferation of $T$. gondii tachyzoites in macrophages (Table 31), increased NO generation in these cells (Table 32) ${ }^{[809]}$, and diminished various tissue pathology in animals infected with the parasite (Table 33), possibly by acting on tachyzoites in parasitophorous vacuole ${ }^{[810]}$. Of note, vitamin D may however also be linked to the increased susceptibility and mortality of mice infected with the pathogen, probably because of its downregulation of the $T_{H} 1$ type cytokine response $\mathrm{e}^{[811]}$.

In summary, experimental and clinical data provide vast evidence that vitamin D have important beneficial impact on immunity, as well as on $T$. gondii infection, and therefore it should find a solid place in various treatment regimens directed against both atherosclerosis and the pathogen.

\section{CONCLUDING REMARKS}

Intracellular parasite $T$. gondii is widely disseminated in animals and infects approximately $30-50 \%$ of human population worldwide. The pathogen attacks almost all nucleated cells, and vascular endothelial cells have increased susceptibility to infection with $T$. gondii causing oxidative stress and endothelial dysfunction. Persistent inflammation associated with the increased production of proinflammatory cytokines and cysteine cathepsins during latent chronic toxoplasmosis cause enhanced generation of foam cells, disturbances of blood coagulation, and development of atherosclerotic lesions. In these bioprocesses, $T$. gondii tachyzoites become an additional source of $T g$ ACAT1 and $T g$ ACAT2 and cathepsin proteases that are superimposing on the respective enzymes produced in the host vascular endothelial cells, thereby enhancing their final activities. These findings may be supported by at least six facts: (a) early stages of atherosclerosis began already in early embryonic life probably caused by congenital toxoplasmosis acquired in the first trimester of gestation as a result of parasite transmission from mother to fetus ${ }^{[110]}$; (b) the parasite penetrated nucleated erythroblasts and macroreticulocytes from fetal mouse liver and the circulating erythrocytes of fetal mice ${ }^{[414]}$, and multiplied in the immature cells ${ }^{[812]}$; (c) the accelerated atherosclerotic development was due to the increased levels of cholesterol and LDL reported in $T$. gondii infected humans ${ }^{[16]}$ and animals ${ }^{[813]}$; (d) the highest amongst analyzed regression coefficients $\left(\mathrm{B}=12.49, p=0.026, \mathrm{Eta}^{2}=0.058\right)$ between the prevalence of toxoplasmosis and cardiovascular (cerebrovascular and ischemic heart) diseases in a set of 88 countries $^{[30]}$; (e) there is a frequent comorbidity of severe carotid atherosclerosis and mood disorders $(p<0.0001)^{[814]}$ as well as a significant relationship between chronic T. gondii infection and mood disturbances ${ }^{[815]}$; and (f) pravastatin, simvastatin and other statins (drugs with hypocholesterolemic and antiatherosclerotic activities) inhibited the adhesion, replication and proliferation of T. gondii ${ }^{[816-819]}$; propranolol, a $\beta$-adrenoreceptor blocking agent, also exerted antitoxoplasmic

Table 30 Effects of 1,25-dihydroxyvitamin $\mathrm{D}_{3}$ on innate immune system (acc. to Pelajo et $^{\text {al }}{ }^{[786]}$; Youssef et al ${ }^{[787]}$; with own modification).

\begin{tabular}{|c|c|}
\hline Vitamin D actions in the immune system & Refs \\
\hline $\begin{array}{l}\text { Decreases the antigen-presenting activity of macrophages to lymphocytes; acts on phenotype and function of antigen presenting } \\
\text { cells (monocytes, macrophages, dendritic cells) via oxidative burst, production of acid phosphatase and hydrogen peroxide, and } \\
\text { maturation of cytokines; acts on neutrophils mobility and phagocytic function; upregulates antimicrobial peptides (cathelicidins, } \\
\text { beta-2/beta-3 defensins) in neutrophils, NK cells, monocytes, macrophages, and lymphocytes }\end{array}$ & [787-789] \\
\hline Inhibits the maturation of monocytes into dendritic cells & {$[790,791]$} \\
\hline Induces the activation of T reg and NK T cells & {$[792,793]$} \\
\hline Inhibits $\mathrm{T}_{\mathrm{H}} 1$ type cytokine response & {$[783,790,791,793]$} \\
\hline Decreases IL-2, IFN- $\gamma$, and CD4 and CD8 DNA synthesis & {$[776,787-791]$} \\
\hline Stimulates the $\mathrm{T}_{\mathrm{H}} 2$ type cytokine dominance & {$[783,790,791,793]$} \\
\hline Increases IL-4, IL-5 and IL-10 synthesis & {$[777,789,790]$} \\
\hline Inhibits the synthesis of IL-12, IL-1, IL-6 and TNF- $\alpha$ & {$[776,788,794,795]$} \\
\hline Inhibits B cell proliferation, plasma cell differentiation, and antibody/immunoglobulin production & {$[789-791,796,797]$} \\
\hline Triggers B cell apoptosis & {$[787]$} \\
\hline Increases apoptosis induced by dendritic cells and T lymphocytes - tolerance & {$[797]$} \\
\hline Induces autophagy in human monocytes/macrophages via cathelicidin & {$[777,780]$} \\
\hline Induces NOS in a human monocyte/macrophage cell line ${ }^{\text {a }}$ & [798] \\
\hline
\end{tabular}

NOS, nitric oxide synthase. ${ }^{\text {a }} \mathrm{NO}$ is an effector molecule of parasite killing ${ }^{[799]}$. Interestingly, $1 \alpha 25(\mathrm{OH})_{2} \mathrm{D}_{3}$ suppressed growth and triggered the destruction and clearance of another intracellular pathogen Mycobacterium tuberculosis by activating autophagy in infected human monocytes/macrophages ${ }^{[800]}$. 
Table 31 Effect of vitamin Dз and IFN-ץ on proliferation of T. gondii (RH strain) tachyzoites per infected peritoneal macrophage of BALB/c mice after incubation for 96 hrs in RPMI1640 cells culture (acc. to Ghaffarifar et al ${ }^{[809]}$; with own modification).

\begin{tabular}{|l|c|c|c|c|c|}
\hline Experiment No. & Controls & Solvent $^{\mathrm{a}}$ & Vit $_{3}$ (1000 IU) & IFN- $\gamma$ (100 IU) & Vit D (1000 IU) plus IFN- $\gamma$ (100 IU) \\
\hline 1 & $3.01 \pm 0.14$ & $2.93 \pm 0.16$ & $2.49 \pm 0.19^{\mathrm{b}}$ & $2.6 \pm 0.2^{\mathrm{b}}$ & $2.37 \pm 0.19^{\mathrm{b}}$ \\
\hline 2 & $3.15 \pm 0.12$ & $3.03 \pm 0.16$ & $2.74 \pm 0.16$ & $2.5 \pm 0.15^{\mathrm{b}}$ & $2.58 \pm 0.13^{\mathrm{b}}$ \\
\hline 3 & $3.05 \pm 0.15$ & $3.04 \pm 0.14$ & $2.82 \pm 0.17$ & $2.57 \pm 0.16^{\mathrm{b}}$ & $2.69 \pm 0.2^{\mathrm{b}}$ \\
\hline 4 & $3.16 \pm 0.14$ & $3.0 \pm 0.14$ & $2.39 \pm 0.19^{\mathrm{b}}$ & $2.59 \pm 0.2^{\mathrm{b}}$ & $2.03 \pm 0.19^{\mathrm{b}}$ \\
\hline
\end{tabular}

Numbers of tachyzoites are given as a mean \pm SD. ${ }^{\text {a }}$ Ethanol $95 .{ }^{\mathrm{b}}$ Statistically significant differences compared with controls $(p \leq 0.05)$.

Table 32 Effect of vitamin $\mathrm{D}_{3}$ and IFN-ץ on NO production by peritoneal macrophages of BALB/c mice infected with T. gondii (RH strain) after incubation for 24 hrs in RPMI1640 cells culture (acc. to Ghaffarifar et al ${ }^{[809]}$; with own modification).

\begin{tabular}{|c|c|c|c|c|c|}
\hline Experiment No. & Controls & Solvent $^{\mathrm{a}}$ & Vit $\mathrm{D}_{3}(1000 \mathrm{IU})$ & IFN- $\gamma(100 \mathrm{IU})$ & Vit $\mathrm{D}_{3}(1000 \mathrm{IU})$ plus IFN- $\gamma$ (100 IU) \\
\hline 1 & $109 \pm 8.02$ & $108.2 \pm 12.45$ & $165 \pm 11.30^{\mathrm{b}}$ & $146 \pm 7.22^{\mathrm{b}}$ & $187.8 \pm 9.82^{b}$ \\
\hline 2 & $108 \pm 9.46$ & $108.9 \pm 6.93$ & $121.2 \pm 6.68$ & $139.5 \pm 5.76^{b}$ & $136.2 \pm 10.21^{b}$ \\
\hline 3 & $109.6 \pm 7.35$ & $108.2 \pm 4.96$ & $139 \pm 7.01^{b}$ & $146 \pm 4.93^{b}$ & $146.9 \pm 9.62^{b}$ \\
\hline 4 & $109 \pm 7.03$ & $108.6 \pm 4.26$ & $166 \pm 7.01^{b}$ & $146.2 \pm 5.60^{b}$ & $191.5 \pm 9.62^{b}$ \\
\hline
\end{tabular}

Values are given as mean \pm SD. ${ }^{\mathrm{a}}$ Ethanol 95 . ${ }^{\mathrm{b}}$ Statistically significant results compared with controls $(p \leq 0.05)$. NO production was estimated as a nitrite release from infected macrophages $(\mu \mathrm{M})$.

Table 33 Effect of pretreatment with 1,25(OH $)_{2} \mathrm{D}_{3}(0.5 \mu \mathrm{g} / \mathrm{kg} / 2$ days $)$ on tissue pathology caused by $T$. gondii avirulent ME49 strain infection with 20 cysts administered intraperitoneally in BALB/c mice (acc. to Rajapakse et $a^{[810]}$; with own modification).

\begin{tabular}{|l|c|c|c|}
\hline \multirow{2}{*}{ Tissue } & Pathology & $\begin{array}{c}\text { No } \\
\text { treatment }\end{array}$ & $\begin{array}{c}\text { Treatment } \\
\text { with Vit D } 3\end{array}$ \\
\hline \multirow{2}{*}{ Lung } & Alveolar macrophages & 1 & 0 \\
\cline { 2 - 4 } & Inflammatory foci & 2 & 1 \\
\hline \multirow{3}{*}{ Liver } & Inflammatory foci & 3 & 2 \\
\cline { 2 - 4 } & Hemorrhage & 2 & 0 \\
\cline { 2 - 4 } & Mitosis & 1 & 0 \\
\hline \multirow{2}{*}{ Small intestine } & Inflammatory infiltrates & 1 & 0 \\
\cline { 2 - 4 } & Necrotic mucosal cells & 2 & 1 \\
\hline Brain & Presence of the parasite & 2 & 0 \\
\hline Spleen & Granulocytes & 2 & 1 \\
\hline
\end{tabular}

Histopathologic examination of the tissues was performed 7 days post inoculation. Numbers are based on severity of the lesions $(0$, no lesion, 1, mild, 2, slight, 3, moderate changes) and the total was divided the number of animals in the group. Also, in vitro studies with incubated intestinal epithelial cells showed a significant dose-dependent inhibition of intracellular T. gondii tachyzoites (RH strain, type I) proliferation at $10^{-7}$ $\mathrm{M}$ of $1,25(\mathrm{OH})_{2} \mathrm{D}_{3}$ concentration.

effects $^{[820,821]}$ and had both antiatherosclerotic ${ }^{[822]}$ and atherogenic ${ }^{[823]}$ properties. Thus, it seems that latent chronic toxoplasmosis play an essential but so far neglected role in the pathogenesis of atherosclerosis.

Finally, one may ask why until now there was no suggestion that this pathogen might be so crucial and successful environmental factor responsible for triggering and development of atherosclerosis on a global scale? Probably, the answer to this question refers to the facts that physicians have a very limited knowledge about this widely disseminated pathogen in humans, animals and birds, and there is a general opinion that such infection is usually harmless in immunocompetent individuals ${ }^{[28,29]}$. Moreover, there is no sufficiently specific and sensitive laboratory method available so far for diagnosis of infection with the parasite, and a plethora of $T$. gondii antigens detected in human plasma due to various parasite strains that infect host, and different stage-specific antigens of the pathogen ${ }^{[824]} . T$. gondii antigens recognized by $\operatorname{IgG}$ antibodies also differ between immunocompetent hosts with and without active proliferation of tachyzoites during chronic infection ${ }^{[825]}$. Recently however, we proposed and gave the reasons ${ }^{[45]}$ for a need to use a very specific method proposed long time ago by Sternberger et a ${ }^{[826]}$ and modified by Conley and Jenkins ${ }^{[827]}$.

\section{REFERENCES}

1 Sessa R, Di Pietro M, Filardo S, Turriziani O. Infectious burden and atherosclerosis: a clinical issue. World J Clin Cases 2014; 2(7): 240-249. [DOI: 10.12998/wjcc.v2.i7.240]; [PMID: 25032197]

2 World Health Organization Global Atlas on Cardiovascular Disease Prevention and Control. Mendis S, Puska P, Norrving B (Eds) Available from: URL: http: // whqlibdoc.who.int/ publications/2011/9789241564373_eng.pdf.

3 World Health Organization. Available from: http: // www.who.int/ cardiovascular_diseases/en/(last accessed 20 June 2014).

4 Nichols M, Townsend N, Scarborough P, Rayner M. Cardiovascular disease in Europe 2014: Epidemiological update. Eur Heart J 2014; 35(42): 2950-2959. [DOI: 10.1093/eurheartj/ ehu299]; [PMID: 25139896]

5 Portugal LR, Fernandes LR, Alvarez-Leite JI. Host cholesterol and inflammation as common key regulators of toxoplasmosis and atherosclerosis development. Expert Rev Anti Infect Ther 2009; 7(7): 807-819. [DOI: 10.1586/eri.09.60]; [PMID: 19735223]

6 Singh A, Neki NS, Bisht S, Singh I, Gupta H. Current advances in understanding the pathogenesis of atherosclerosis and its clinical implications in coronary artery disease. JIMSA 2012; 25(4): 25154.

7 Hansson GK. Inflammation, atherosclerosis, and coronary artery disease. N Engl J Med 2005; 352(16): 1685-1695. [DOI: 10.1056/ NEJMra043430]; [PMID: 15843671]

8 Tabas I, Williams KJ, Boren J. Subendothelial lipoprotein retention as the initiating process in atherosclerosis: Update and therapeutic implications. Circulation 2007; 116(16): 18321844. [DOI: 10.1161/CIRCULATIONAHA.106676890]; [PMID: $17938300]$

9 Ketelhuth DFJ, Hansson GK. Modulation of autoimmunity and atherosclerosis - common targets and promising translational approaches against disease. Circ $J$ 2015; 79(5): 924-933. [DOI: 10.1253/circj.CJ-15-0167]; [PMID: 25766275]

10 Rafieian-Kopaei M, Setorki M, Doudi M, Baradaran A, Nasi H. Atherosclerotic process, indicators, risk factors and new hopes. Int J Prev Med 2014; 5(8): 927-946]; [PMID: 25489440]

11 Mangge H, Becker K, Fuchs D, Gostner JD. Antioxidants, inflammation and cardiovascular disease. World J Cardiol 2014; 6(6): 462-477. [DOI: 10.4330/wjc.v6.i6.462]; [PMID: 24976919]

12 Peluzio MC, Homem AP, Cesar GC, Azevedo GS, Amorim R, Cara DC, et al. Influences of alpha-tocopherol on cholesterol metabolism and fatty streak development in apolipoprotein 
E-deficient mice fed an atherogenic diet. Braz J Med Biol Res 2001; 34(12): 1539-1545]; [PMID: 11717706]

13 Bobryshev YV, Lord RS. Identification of natural killer cells in human atherosclerotic plaque. Atherosclerosis 2005; 180(2): 423-427. [DOI: 10.1016/j.atherosclerosis.2005.01.046]; [PMID: 15910872]

14 Ait-Oufella H, Sage AP, Mallat Z, Tedgui A. Adaptive (T and B cells) immunity and control by dendritic cells in atherosclerosis. Circ Res 2014; 114(10): 1640-1660. [DOI: 10.1161/ CIRCRESAHA.114.302761

15 Ammirati E, Moroni E, Magnoni M, Camici PG. The role of T and B cells in human atherosclerosis and atherothrombosis. Clin Exp Immunol 2014; 179(2): 173-187. [DOI: 10.1111/cei.12477]; ]; [PMID: 25352024]

16 Flegr J, Priplatova L, Hampl R, Bicikovia M, Ripova D, Mohr P. Difference of neuro- and immunomodulatory steroids and selected hormone and lipid concentrations between Toxoplasma-free and Toxoplasma infected but not CMV-free and CMV-infected schizophrenia patients. Neuro Endocrinol Lett 2014; 35(1): 20 27]; [PMID: 24625913]

17 De Boer OJ, van der Wal AC, Becker AE. Atherosclerosis, inflammation, and infection. J Pathol 2000; 190(3): 237-243. [DOI: 10.1002/(SICI)1096-9896(200002)190: 3<237: : AIDPATH541>3.0.CO: 2-N]; [PMID: 10685058]

18 Rupprecht HJ, Blankerberg S, Bickel C, Rippin G, Hafner G, Prellwitz W, et al. Impact of viral and bacterial infectious burden on long-term prognosis in patients with coronary artery disease. Circulation 2001; 104(1): 25-31. [DOI: 10.1161/hc2601.091703]; [PMID: 11435333]

19 Stoll LL, Denning GM, Weintraub NL. Potential role of endotoxin as a pro-inflammatory mediator of atherosclerosis. Arterioscler Thromb Vasc Biol 2004; 24(12): 2227-2236. [DOI: 10.1161/01. ATV.0000147534.69062.dc]; [PMID: 15472123]

20 Conti M, Sanna F, Farci GAM, Uda S, Porcu G, Collu M, et al. Abnormal macrophage response to microbial stimulus in a 43-year-old man with severe form of atherosclerosis: a case report. $J$ Med Case Rep 2010; 4: 183. [DOI: 10.1186/1752-1947-4-183]; [PMID: 20565856]

21 Filardo S, Di Pietro M, Farcomeni A, Schiavoni G, Sessa R. Chlamydia pneumoniae-mediated inflammation in atherosclerosis: a meta-analysis. Mediators Inflamm 2015; article ID 378658 [DOI: 10.1155/2015/378658]; [PMID: 26346892]

22 Stoner L, Lucero AA, Palmer BR, Jones LM, Young JM, Faulkner J. Inflammatory biomarkers for predicting cardiovascular disease. Clin Biochem 2013; 46(15): 1353-1371. [DOI: 10.1016/ j.clinbiochem.2013.05.070]; [PMID: 23756129]

23 Pant S, Deshmukh A, Gurumurthy S, Pothineni NV, Watts TE, Romeo F, et al. Inflammation and atherosclerosis - revisited. J Cardiovasc Pharmacol Ther 2014; 19(2): 170-178. [DOI: 10.1177/1074248413504994]; [PMID: 24177335]

24 Furtado JM, Smith JR, Belfort R Jr, Gattey D, Winthrop KL. Toxoplasmosis: a global threat. J Glob Infect Dis 2011; 3(3): 281284. [DOI: 10.4103/0974-777X.83536]; [PMID: 21887062]

25 Torgerson PR, Mastroiacovo P. The global burden of congenital toxoplasmosis: a systematic review. Bull World Health Organ 2013; 91(7): 501-508. [DOI: 10.2471/BLT.12.111732]; [PMID: 23825877]

26 Klaren VN, Kijlstra A. Toxoplasmosis, an overview with emphasis on ocular involvement. Ocul Immunol Inflamm 2002; 10(1): 1-26]; [PMID: 12461700]

27 Hinze-Selch D, Däubener W, Erdag S, Wilms S. The diagnosis of a personality disorder increases the likelihood for seropositivity to Toxoplasma gondii in psychiatric patients. Folia Parasitol (Praha) 2010; 57(2): 129-135]; [PMID: 20608475]

28 Halonen SK, Weiss LM. Toxoplasmosis. Handbook of Clinical Neurology 2013; 114: 125-145. [DOI: 10.1016/B978-0-44453490-00008-X]
29 McLeod R, Van Tubbergen C, Montoya JG, Petersen E. Human Toxoplasma Infection. In: Weiss LM, Kim K (eds), Toxoplasma Gondii (Second Edition), Academic Press, Amsterdam 2013; pp. 99-159. ISBN 9780123964816

30 Flegr J, Prandota J, Sovičková M, Israili ZH. Toxoplasmosis - a global threat. Correlation of latent toxoplasmosis with specific disease burden in a set of 88 countries. PLOS ONE 2014; 9(3): e90203. [DOI: 10.1371/journal.pone.0090203]; [PMID: 24662942]

31 Robben PM, Mordue DG, Truscott SM, Takeda K, Akira S, Sibley LD. Production of IL-12 by macrophages infected with Toxoplasma gondii depends on the parasite genotype. J Immunol 2004; 172(6): 3686-3694. [DOI: 10.4049/jimmunol.172.6.3686]; [PMID: 15004172]

32 Gazzinelli RT, Amichay D, Scharton-Kersten T, Grunwald E, Farber JM, Sher A. Role of macrophage-derived cytokines in the induction and regulation of cell-mediated immunity to Toxoplasma gondii. In: Gross U (ed) Toxoplasma gondii. Curr Top Microbiol Immunol 1996; 219: 127-139]; [PMID: 8791695]

33 Mordue DG, Monroy F, La Regina M, Dinarello CA, Sibley LD. Acute toxoplasmosis leads to lethal overproduction of Th1 cytokines. J Immunol 2001; 167(8): 4574-4584. [DOI: 10.4049/ jimmunol.167.8.4574]; [PMID: 11591786]

34 Gavrilescu LC, Denkers EY. IFN- $\gamma$ overproduction and high level apoptosis are associated with high but not low virulence Toxoplasma gondii infection. J Immunol 2001; 167(2): 902-99. [DOI: 10.4049/jimmunol.167.2.902]; [PMID: 11441097]

35 Boothroyd JC, Grigg ME. Population biology of Toxoplasma gondii and its relevance to human infection: do different strains cause different disease. Curr Opin Microbiol 2002; 5(4): 438-442. [DOI: 10.1016/S1369-5274(02)00349-1]; [PMID: 12160866]

36 Cines DB, Pollak ES, Buck CA, Loscalzo J, Zimmerman GA, McEver RP, et al. Endothelial cells in physiology and in the pathophysiology of vascular disorders. Blood 1998; 91(10): 3527 3561. [PMID: 9572988]

37 Baumgartner-Parzer SM, Waldhäusl WK. The endothelium as a metabolic and endocrine organ; its relation with insulin resistance. Exp Clin Endocrinol Diabetes 2001; 109 Suppl 2: S166-S179. [DOI: 10.1055/s-2001-18579]; [PMID: 11460568]

38 Dimmeler S, Zeiher AM. Endothelial cell apoptosis in angiogenesis and vessel regression. Circ Res 2000; 87(6): 434439. [DOI: 10.1161/01.RES.87.6.434]; [PMID: 10988233]

39 Dimmeler S, Haendeler J, Zeiher AM. Regulation of endothelial cell apoptosis in atherothrombosis. Curr Opin Lipidol 2002; 13(5): 531-536]; [PMID: 12352017]

40 Ross R. Atherosclerosis - an inflammatory disease. $N$ Engl J Med 1999; 340(2): 115-126. [DOI: 1056/NEJM199901143400207]; [PMID 9887164]

41 Hadi H, Carr C, Suwaidi J. Endothelial dysfunction: cardiovascular risk factors, therapy, and outcome. Vasc Health Risk Manage 2005; 1(3): 183-198]; [PMID: 17319104]

42 Couillard C, Ruel G, Archer WR, Pomerleau S, Bergeron J, Couture $\mathrm{P}$, et al. Circulating levels of oxidative stress markers and endothelial adhesion molecules in men with abdominal obesity. J Clin Endocrinol Metab 2005; 90(12): 6454-6459. [DOI: 1210/ jc.2004-2438]; [PMID: 16189262]

42a Charakida M, Masi S, Lüscher T, Kastelein JJP, Deanfield JE. Assessment of atherosclerosis: the role of flow-mediated dilation. Eur Heart J 2010; 31(23): 2854-2861. [DOI: 10.1093/eurheartj/ ehq340]; [PMID: 20864485]

42 b Green DJ, Jones H, Thijssen D, Cable NT, Atkinson G. Flowmediated dilation and cardiovascular event prediction. Does nitric oxide matter?. Hypertension 2011; 57(3): 363-369. [DOI: 10.1161/ HYPERTENSIONAHA.110.167015]; [PMID: 21263128]

42c Ciccone MM, Bilianou E, Balbarini A, Gesualdo M, Ghiadoni L, Metra M, et al. Task force on: Early markers of atherosclerosis: influence of age and sex. J Cardiovasc Med (Hagerstown) 2013; 
14(10): 757-766. [DOI: 10.2459/JCM.0b013e328362078d]; [PMID: 24335886]

42d Hopkins ND, Dengel DR, Stratton G, Kelly AS, Steinberger J, Zavala $\mathrm{H}$, et al. Age and sex relationship with flow-mediated dilation in healthy children and adolescents. J Appl Physiol 2015: 119(8): 926-933. [DOI: 10.1152/japplphysiol.01113.2014]; [PMID: 26251515]

42e Beyer AM, Zinkevich N, Miller B, Liu Y, Wittenburg AL, Mitchell $\mathrm{M}$, et al. Transition in the mechanism of flow-mediated dilation with aging and development of coronary artery disease. Basic Res Cardiol 2017; 112(1): 5 [DOI: 10.1007/s00395-016-0594-x]; [PMID: 27995364]

$42 f$ Drager LF, Bortolotto LA, Krieger EM, Lorenzi-Filho G. Additive effects of obstructive sleep apnea and hypertension on early markers of carotid atherosclerosis. Hypertension 2009; 53(1): 6469. [DOI: 10.1161/HYPERTENSIONAHA.108.119420]; [PMID: 19015401]

$42 \mathrm{~g}$ Damiani MF, Zito A, Carratu P, Falcone VA, Bega E, Scicchitano $\mathrm{P}$, et al. Obstructive sleep apnea, hypertension, and their additive effects on atherosclerosis. Biochem Res Int 2015; 2015: 984193. [DOI: 10.1155/2015/984193]; [PMID: 26697221]

42h Yasunaga T, Ikeda S, Koga S, Nakata T, Yoshida T, Maruda N, et al. Plasma pentraxin 3 is a more potent predictor of endothelial dysfunction than high-sensitive C-reactive protein. Int Heart $J$ 2014; 55(2): 160-164. [DOI: 10.1536/ihj.13-253]; [PMID: 24632958]

42i dell'Oglio MP, Simone S, Ciccone M, Corciulo R, Gesualdo M, Zito A, et al. Neutrophil-dependent pentraxin-3 and reactive oxygen species production modulate endothelial dysfunction in hemodialysis patients. Nephrol Dial Transplant 2016; Dec 3. [DOI: 10.1093/ndt/gfw363]; [PMID: 27915246]

43 Channon JY, Seguin RM, Kasper LH. Differential infectivity and division of Toxoplasma gondii in human peripheral blood leukocytes. Infect Immun 2000; 68(8): 4822-4826. [PMID: 10899898]

44 Nakao M, Konishi E. Proliferation of Toxoplasma gondii in human neutrophils in vitro. Parasitology 1991; 103 Pt 1: 23-27]; [PMID: 1658717]

45 Wilson CB, Remington JS. Activity of human blood leukocytes against Toxoplasma gondii. J Infect Dis 1979; 140(6): 890-895]; [PMID: 541523]

46 Delemarre FG, Stevenhagen A, Kroon FP, Vaneer MY, Meenhorst PL, van Furth R. Effect of IFN- $\gamma$ amma on the proliferation of Toxoplasma gondii in monocytes and monocyte-derived macrophages from AIDS patients. Immunology 1994; 83(4): 646650. [PMID: 7875745]

47 Fadul CE, Channon JY, Kasper LH. Survival of immunoglobulin G-opsonized Toxoplasma gondii in nonadherent human monocytes. Infect Immun 1995; 63(11): 4290-3294. [PMID: 7591060]

48 McLeod R, Bensch KG, Smith SM, Remington JS. Effects of human peripheral blood monocytes, monocyte-derived macrophages, and spleen mononuclear phagocytes on Toxoplasma gondii. Cell Immunol 1980; 54(2): 330-350. [PMID: 7418010]

49 Murray HW, Rubin BY, Carriero SM, Harris AM, Jaffee EA. Human mononuclear phagocyte antiprotozoal mechanisms: oxygen-dependent vs oxygen-independent activity against intracellular Toxoplasma gondii. J Immunol 1985; 134(3): $1982-$ 1988. [PMID: 2981929]

50 Murray HW, Szuro-Sudol A, Wellner D, Oca MJ, Granger AM, Libby DM, et al. Role of tryptophan degradation in respiratory burst-independent activity antimicrobial activity of gamma interferon-stimulated human macrophages. Infect Immun 1989; 57(3): 845-849. [PMID: 2492973]

51 Wilson CB, Westall J. Activation of neonatal and human macrophages by alpha, beta, and gamma interferons. Infect Immun 1985; 49(2): 351-356. [PMID: 3926648]
52 Anderson SE, Bautista S, Remington SJ. Induction of resistance to Toxoplasma gondii in human macrophages by soluble lymphocyte products. $J$ Immunol 1976; 117(2): 381-387. [PMID: 950460]

53 Halonen SK, Lyman WD, Chiu FC. Growth and development of Toxoplasma gondii in human neurons and astrocytes. J Neuropathol Exp Neurosci 1996; 55(11): 1150-1156. [DOI: 10.1091/00005072-199611000-00006]; [PMID: 8939198]

54 Pfefferkorn ER. Interferon g blocks the growth of Toxoplasma gondii in human fibroblasts by inducing the host cells to degrade tryptophan. Proc Natl Acad Sci USA 1984; 81(3): 908-912. [PMID: 6422465]

55 Pfefferkorn ER, Guyre PM. Inhibition of growth of Toxoplasma gondii in cultured fibroblasts by human recombinant gamma interferon. Infect Immun 1984; 44(2): 211-216. [PMID: 6425215]

56 Woodman JP, Dimier IH, Bout DT. Human endothelial cells are activated by IFN- $\gamma$ to inhibit Toxoplasma gondii replication. Inhibition is due to a different mechanism from that existing in mouse macrophages and human fibroblasts. J Immunol 1991; 147(6): 2019-2023. [PMID: 1909738]

57 Nagineni CN, Pardhasaradhi K, Martins MC, Detrick B, Hooks JJ. Mechanism of interferon-induced inhibition of Toxoplasma gondii replication in human retinal pigment epithelial cells. Infect Immun 1996; 64(10): 4188-4196. [PMID: 8926087]

58 Peterson PK, Gekker G, Hu S, Chao CC. Human astrocytes inhibit intracellular multiplication of Toxoplasma gondii by a nitric oxide-mediated mechanism. $J$ Infect Dis 1995; 171(2): 516-518. [PMID: 7844409]

59 Chao CC, Gekker RG, Hu S, Peterson PK. Human microglial cell defense against Toxoplasma gondii. The role of cytokines. $J$ Immunol 1994; 152(3): 1246-1252. [PMID: 8301129]

60 Canedo-Solares I, Calzada-Ruiz M, Ortiz-Alegria LB, OrtizMuniz AR, Correa D. Endothelial cell invasion by Toxoplasma gondii: differences between cell types and parasite strains. Parasitol Res 2013; 112(8): 3029-3033. [DOI: 10.1007/s00436013-3476-2]; [PMID: 23749089]

61 Dimier IH, Bout DT. Inhibitory effect of interferon-gamma activated ovine umbilical vein endothelial cells on the intracellular replication of Toxoplasma gondii. Vet Res 1996; 27(4-5): 527-534. [PMID: 8822620]

62 Smith JR, Franc DT, Carter NS, Zamora D, Planck SR, Rosenbaum JT. Susceptibility of retinal vascular endothelium to infection with Toxoplasma gondii tachyzoites. Invest Ophthalmol Visual Sci 2004; 45(4): 1157-1161. [PMID: 15037582]

63 Furtado JM, Bharadwaj AS, Chipps TJ, Pan Y, Ashander LM, Smith JR. Toxoplasma gondii tachyzoites cross retinal endothelium assisted by intercellular adhesion molecule- 1 in vitro. Immunol Cell Biol 2012; 90(9): 912-915. [DOI: 10.1038/ icb.2012.21]; [PMID: 22525368]

64 Shin D, Garcia-Cardena G, Hayashi S, Gerety S, Asahara T, Stavrakis G, et al. Expression of ephrinB2 identifies a stable genetic difference between arterial and venous vascular smooth muscle as well as endothelial cells, and marks subsets of microvessels at sites of adult neovascularization. Dev Biol 2001; 230(2): 139-150. [DOI: 10.1006/dbio.2000.9957]; [PMID: $11161568]$

65 Ruoslahti E, Rajotte D. An address system in the vasculature of normal tissues and tumors. Annu Rev Immunol 2000; 18: 813-827. [DOI: 10.1146/annurev.immunol.18.1.813]; [PMID: 10837076]

66 Ghitescu L, Robert M. Diversity in unity: the biochemical composition of the endothelial cell surface varies between the vascular beds. Microsc Res Tech 2002; 57(5): 38138-38139. [DOI: 10.1002/jemt.10091]; [PMID: 12112444]

67 Peterson PK, Gekker G, Hu S, Chao CC. Human astrocytes inhibit intracellular multiplication of Toxoplasma gondii by a nitric oxide-mediated mechanism. $J$ Infect Dis 1995; 171(2): 516-518]; [PMID: 7844409] 
68 Courret N, Darche S, Sonigo P, Milon G, Buzoni Gatel D, Tardieux I. CD11c- and CD11b-expressing mouse leukocytes transport single Toxoplasma gondii tachyzoites to the brain. Blood 2006; 107(1): 309-316. [DOI: 10.1182/blood-2005-02-0666]; [PMID: 16051744]

69 Furtado JM, Bharadwaj AS, Ashander LM, Olivas A, Smith JR. Migration of Toxoplasma gondii-infected dendritic cells across human retinal vascular endothelium. Invest Ophthalmol Vis Sci 2012; 53(11): 6856-6862. [DOI: 10.1167/iovs.12-10384]; [PMID: 22952125]

70 Flaherty ML, Flemming KD, McClelland R, Jorgensen NW, Brown RD Jr. Population-based study of symptomatic internal carotid artery occlusion : incidence and long-term follow-up. Stroke 2004; 35(8): e349-352. [DOI: 10.1161/01. STR.00001350024.54608.3f]; [PMID: 15232124]

71 Cunningham KS, Gotlieb AI. The role of shear stress in the pathogenesis of atherosclerosis. Lab Invest 2005; 85(1): 9-23. [DOI: 10.1038/labinvest.3700215]; [PMID: 15568038]

72 Jenkins NT, Padilla J, Boyle LJ, Credeur DP, Laughlin MH, Fadel PJ. Disturbed blood flow acutely induces activation and apoptosis of the human vascular endothelium. Hypertension 2013; 61(3): 615-621. [DOI: 10.1161/HYPERTENSIONAHA.111.00561]; [PMID: 23319545]

73 Warboys CM, Amini N, de Luca A, Evans PC. The role of blood flow in determining the sites of atherosclerotic plaques. F1000 Med Rep 2011; 3: 5. [DOI: 10.3410/M3-5]; [PMID: 21654925]

74 Mullick AE, Soldau K, Kiosses WB, Bell III TA, Tobias PS, Curtiss LK. Increased endothelial expression of Toll-like receptor 2 at sites of disturbed blood flow exacerbates early atherogenic events. J Exp Med 2008; 205(2): 373-83. [DOI: 10.1084/ jem.20071096]; [PMID: 18250194]

75 Curtiss LK, Tobias PS. Emerging role of Toll-like receptors in atherosclerosis. J Lipid Res 2009; 50 Suppl: S340-S345. [DOI: 10.1194/jlr.R800056-JLR200]; [PMID: 18980945]

76 Bendall JK, Douglas G, McNeill E, Channon KM, Crabtree MJ. Tetrahydrobiopterin in cardiovascular health and disease. Antioxid Redox Signal 2014; 20(18): 3040-3077. [DOI: 10.1089/ ars.2013.5566]; [PMID: 24294830]

77 Ignarro LJ. Nitric oxide as a unique signaling molecule in the vascular system: a historical overview. J Physiol Pharmacol 2002; 53(4 Pt 1): 503-514]; [PMID: 12512688]

78 Harker KS, Ueno N, Wang T, Bonhomme C, Liu W, Lodoen MB. T. gondii modulates the dynamics of human monocyte adhesion to vascular endothelium under fluidic shear stress. J Leukoc Biol 2013; 93(5): 789-800. [DOI: 10.1189/jlb.1012517]; [PMID: 23485448

79 Harker KS, Jivan E, McWhorter FY, Liu WF, Lodoen MB. Shear forces enhance Toxoplasma gondii tachyzoites motility on vascular endothelium. $m B I O$ 2014; 5(2): e01111-01113. [DOI: 10.1128/mBio.01111-13]

80 Konradt C, Ueno N, Christian DA, Delong JH, Pritchard GH, Herz J, et al. Endothelial cells are a replicative niche for entry of Toxoplasma gondii to the central nervous system. Nat Microbiol 2016; 1: 16001. [DOI: 10.1038/nmicrobiol.2016.1]; [PMID: 27572166]

81 Herrera MD, Mingorance C, Rodriguez-Rodriguez R, de Sotomayor MA. Endothelial dysfunction and aging: An update. Ageing Res Rev 2010; 9(2): 142-152. [DOI: 10.1016/ j.arr.2009.07.002]; [PMID: 19619671]

82 Hansson GK, Hellstrand M, Rymo L, Rubbia L, Gabbiani G. Interferon-g inhibits both proliferation and differentiation-specific a-smooth muscle actin in arterial smooth muscle cells. $J$ Exp Med 1989; 170(5): 1595-1608. [DOI: 10.1084/jem.170.5.1595]; [PMID: 2509626]

83 Hansson GK, Libby P, Schönbeck U, Yan Z-Q. Innate and adaptive immunity in the pathogenesis of artheriosclerosis. Circ Res 2002; 91(4): 281-291. [DOI: 10.1161/01.RES.0000029784.15893.10];
[PMID: 12193460]

84 Amento EP, Ehsani N, Palmer H, Libby P. Cytokines positively and negatively regulate interstitial collage gene expression in human vascular smooth muscle cells. Arteriosclerosis 1991; 11(5): 1223-1230. [DOI: 10.1161/01.ATV.11.5.1223]; [PMID: 1911708]

85 Mach F, Schönbeck U, Sukhova GK, Bourcier T, Bonnefoy J-Y, Pober JS, et al. Functional CD 40 ligands expressed on human vascular endothelial cells, smooth muscle cells, and macrophages : implications for CD40-CD40 ligand signaling in atherosclerosis. Proc Natl Acad Sci USA 1997; 94(5): 1931-1936]; [PMID: 9050882]

86 Ridker PM, Rifai N, Stampfer MJ, Hennekens CH. Plasma concentration of interleukin- 6 and the risk of future myocardial infarction among apparently healthy men. Circulation 2000; 101(15): 1767-1772. [DOI: 10.1161/01.CIR.101.15.1767]; [PMID: 10769275]

87 Jonasson L, Holm J, Skalli O, Bondjers G, Hansson GK. Regional accumulation of $\mathrm{T}$ cells, macrophages, and smooth muscle cells in the human atherosclerotic plaque. Arteriosclerosis 1986; 6(2): 131-138. [DOI: 10.1161/01.ATV.6.2.131]; [PMID: 2937395]

88 Stroes E, Kastelein J, Cosentino F, Erkelens W, Wever R, Koomans $\mathrm{H}$, et al. Tetrahydrobiopterin restores endothelial function in hypercholesterolemia. J Clin Invest 1997; 99(1): 4146. [DOI: 10.1172/JCI119131]; [PMID: 9011574]

89 Mäki-Petäjä KM, Day L, Cheriyan J, Hall FC, Östör AJK, Shenker N, et al. Tetrahydrobiopterin supplementation improves endothelial function but does not alter aortic stiffness in patients with rheumatoid arthritis. J Am Heart Assoc 2016; 5(2): e002762. [DOI: 10.1161/JAHA.115.002762]; [PMID: 26896473]

90 Landmesser U, Hornig B, Drexler H. Endothelial function. A critical determinant in atherosclerosis? Circulation 2004; 109(21 Suppl 1): II27-II133. [DOI: 10.1161/CIR.0000129501.88485.1f]; [PMID: 15173060]

91 Rosenkranz-WeissP, Sessa WC, Milstien S, Kaufman S, Watson CA, Pober JS. Regulation of nitric oxide synthesis by proinflammatory cytokines in human umbilical vein endothelial cells. Elevations in tetrahydrobiopterin levels enhance endothelial nitric oxide synthase specific activity. J Clin Invest 1994; 93(5): 2236-2243. [DOI: 10.1172/JCI117221]; [PMID: 7514193]

92 Medzhitov R, Janeway CA Jr. Innate immunity. $N$ Eng J Med 2000; 343(5): 338-344. [DOI: 10.1056/NEJM200008033430506]; [PMID: 10922424]

93 Janeway CA Jr, Medzhitov R. Innate immune recognition. Annu Rev Immunol 2002 ; 20: 197-216. [DOI: 10.1146/annurev. immunol.20.083001.084359]; [PMID: 11861602]

94 Kol A, Bourcier T, Lichtman AH, Libby P. Chlamydial and human heat shock protein 60 s activate human vascular endothelium, smooth muscle cells, and macrophages. J Clin Invest 1999; 103(4): 571-577. [DOI: 10.1172/JCI5310]; [PMID: 10021466]

95 Kol A, Sukhova GK, Lichtman AH, Libby P. Chlamydial heat shock protein 60 localizes in human atheroma and regulates macrophage tumor necrosis factor-alpha and matrix metalloproteinase expression. Circulation 1998; 98(4): 300-307. [DOI: 10.1161/01.CIR.98.4.300]; [PMID: 9711934]

96 Streblow DN, Soderberg-Naucler C, Vieira J, Smith P, Wakabayashi E, Ruchti F, et al. The human cytomegalovirus chemokine receptor US28 mediates vascular smooth muscle cell migration. Cell 1999; 99(5): 511-520. [DOI: 10.1016/S00928674(00)81539-1]; [PMID: 10589679]

97 Zhou J, Quyyumi AA, Norman JE, Csako G, Waclawiw MA, Shearer GM, et al. Effects of total pathogen burden on coronary artery disease risk and C-reactive protein levels. Am J Cardiol 2000; 85(2): 140-146. [DOI: 10.1016/S0002-9149(99)00653-0]; [PMID: 10955367]

98 Espinola-Klein C, Rupprecht HJ, Blankenberg S, Bickel C, Kopp $\mathrm{H}$, Victor A, et al. Impact of infectious burden on progression of carotid atherosclerosis. Stroke 2002; 33(11): 2581-2586. [DOI: 
10.1161/o1.STR.0000034789.82859.A4]; [PMID: 12411646]

99 Espinola-Klein C, Rupprecht HJ, Blankenberg S, Bickel C, Kopp $\mathrm{H}$, Rippin $\mathrm{G}$, et al. Impact of infectious burden on extent and long-term prognosis of atherosclerosis. Circulation 2002; 105(1): 15-21. [DOI: 10.1161/hc0102.101362]; [PMID: 11772870]

100 Elkind MS, Ramakrishnan P, Moon YP, Boden-Albala B, Liu $\mathrm{KM}$, Spitalnik SL, et al. Infectious burden and risk of stroke: the northern Manhattan study. Arch Neurol 2010; 67(1): 33-38. [DOI: 10.1001/archneurol.2009.271]; [PMID: 19901154]

101 Nazmi A, Diez-Roux AV, Jenny NS, Tsai MY, Szklo M, Aiello AE. The influence of persistent pathogens on circulating levels of inflammatory markers: a cross-sectional analysis from the MultiEthnic Study of Atherosclerosis. BMC Public Health 2010; 10: 706. [DOI: 10.1186/1471-2458-10-706]; [PMID: 21083905]

102 Rosenfeld ME, Campbell LA. Pathogens and atherosclerosis: update on the potential contribution of multiple infectious organisms to the pathogenesis of atherosclerosis. Thromb Haemost 2011; 106(5): 858-867. [DOI: 10.1160/TH11-06-0392]; [PMID: 22012133]

103 Prasad A, Zhu J, Halcox JP, Waclawiw MA, Epstein SE, Quyyumi AA. Predisposition to atherosclerosis by infections: role of endothelial dysfunction. Circulation 2002; 106(2): 184-190. [DOI: 10.1161/01.CIR.0000021125.83697.21]; [PMID: 12105156

104 Hulsmans M, Holvoet P. The vicious circle between oxidative stress and inflammation in atherosclerosis. J Cell Mol Med 2010; 14(1-2): 70-78. [DOI: 10.1111/j.1582-4934.2009.00978.x]; [PMID: 19968738]

105 Khairy P, Rinfret S, Tardif J-C, Marchand R, Shapiro S, Brophy $\mathrm{J}$, et al. Absence of association between infectious agents and endothelial function in healthy young men. Circulation 2003; 107(15): 1966-1971. [DOI: 10.1161/01. CIR.0000064895.89033.97]; [PMID: 12681997]

106 Ferrari M, Poli A, Olivieri M, Tardivo S, Biasin C, Balestreri F, et al. Seroprevalence of Chlamydia pneumoniae antibodies in a young adult population sample living in Verona. European Community Respiratory Health Survey (ECRHS) Verona. Infection 2000; 28(1): 38-41]; [PMID: 10697790]

107 Cohen JI. Epstein-Barr virus infection. N Engl J Med 2000; 343(7): 481-492. [DOI: 10.1056/NEJM200008173430707]; [PMID: 10944566]

108 Gattone M, Iacoviello L, Colombo M, Castelnuovo AD, Soffiantino F, Gramoni A, et al. Chlamydia pneumoniae and cytomegalovirus seropositivity, inflammatory markers, and the risk of myocardial infarction in a young age. Am Heart $J$ 2001; 142(4): 633-640. [DOI: 10.1067/mhj.2001.118118]

109 Matsuura E, Atzeni F, Sarzi-Puttini P, Turiel M, Lopez LR, Nurmohamed MT. Is atherosclerosis an autoimmune disease? BMC Medicine 2014; 12: 47. [DOI: 10.1186/1741-7015-12-47]; [PMID: 24642015]

110 Uslu B, Cakmak YO, Sehirli U,Keskinoz EN, Cosgun E, Arbak $\mathrm{S}$, et al. Early onset of atherosclerosis of the carotid bifurcation in newborn cadavers. J Clin Diagn Res 2016; 10(5): ACO1-ACO5. [DOI: 10.7860/JCDR/201619827.7706]; [PMID: 27437199]

111 Sitia S, Tomasoni L, Atzeni F, Ambrosio G, Cordiano C, Catapano A, et al. From endothelial dysfunction to atherosclerosis. Autoimmune Rev 2010; 9(12): 830-834. [DOI: 10.1016/ j.autrev.2010.07.016]; [PMID: 206786595]

112 Hahn BH, Grossman J, Chen W, McMahon M. The pathogenesis of atherosclerosis in autoimmune rheumatic diseases: roles of inflammation and dyslipidemia. J Autoimm 2007; 28(2-3): 69-75. [DOI: 10.1016/j.jaut.2007.02.004]; [PMID: 17433865]

113 Peters MJ, van Halm VP, Nurmohamed MT, Damoiseaux J, Tervaert JW, Twisk JW, et al. Relations between autoantibodies against oxidized low-density lipoprotein, inflammation, subclinical atherosclerosis, and cardiovascular disease in rheumatoid arthritis. $J$ Rheumatol 2008; 35(8): 1495-1499]; [PMID: 18597411]

114 Soeki T, Sata M. Inflammatory biomarkers and atherosclerosis. Int
Heart J 2016; 57(2): 134-139. [DOI: 10.1536/ihj.15-346]; [PMID: 26973275]

115 Matsuo Y, Kubo T, Okumoto Y, Ishibashi K, Komukai K, Tanimoto $\mathrm{T}$, et al. Circulating malondialdehyde-modified lowdensity lipoprotein levels are associated with the presence of thincap fibroatheromas determined by optical coherence tomography in coronary artery disease. Eur Heart J Cardiovasc Imaging 2013; 14(1): 43-50. [DOI: 10.1093/ehjci/jes094]; [PMID: 22573905]

116 Bardag-Gorce F, Li J, French BA, French SW. The effect of ethanol-induced CYP2E1 on proteasome activity: the role of 4-hydroxynonenal. Exp Mol Pathol 2005; 78(2): 109-115. [DOI: 10.1016/j.yexmp.2004.10.005]; [PMID: 15713435]

117 Martinet W, De Meyer GR. Autophagy in atherosclerosis. A cell survival and death phenomenon with therapeutic potential. Circ Res 2008; 104(3): 304-317. [DOI: 10.1161/ CIRCRESAHA.108.188318]; [PMID: 19213965]

118 Martinet W, De Bie M, Schrijvers DM, De Meyer GRY, Herman AG, Kockx MM. 7-ketocholesterol induces protein ubiquitination, myelin figure formation, and light chain 3 processing in vascular smooth muscle cells. Arterioscler Thromb Vasc Biol 2004; 24(12): 2296-2301. [DOI: 10.1161/01.ATV.0000146266.65820.a1]; [PMID: 15458974]

119 Sakakura K, Nakano M, Otsuka F, Ladich E, Kolodgie FD, Virmani R. Pathophysiology of atherosclerosis plaque progression. Heart Lung Circ 2013; 22(6): 399-411. [DOI: 10.1016/ j.hic.2013.03.001]; [PMID: 23541627]

120 Steinberg D. Atherogenesis in perspective: hypercholesterolemia and inflammation as partners in crime. Nat Med 2002; 8(11): 1211-1217. [DOI: 10.1038/nm1102-1211]; [PMID: 12411947]

121 Witztum JL, Steinberg D. The oxidative modification hypothesis of atherosclerosis: does it hold for humans? Trends Cardiovasc Med 2001; 11(3-4): 93-102. [DOI: 10.1016/S1050-1738(01)001116]; [PMID: 11686009]

122 Kiffin R, Bandyopadhyay U, Cuervo AM. Oxidative stress and autophagy. Antioxid Redox Signal (2006) 8(1-2): 152-162. [DOI: 10.1089/ars.2006.8.152]; [PMID: 16487049]

123 Patel RP, Moellering D, Murphy-Ullrich J, Jo H, Beckman JS, Darley-Usmar VM. Cell signaling by reactive nitrogen and oxygen species in atherosclerosis. Free Radical Biol Med 2000; 28(12): 1780-1794]; [PMID: 10946220]

124 Akyol O, Zöröglu SS, Armutcu F, Sahin S, Gurel A. Nitric oxide as a physiopathological factor in neuropsychiatric diseases. In Vivo 2004; 18(3): 377-390]; [PMID: 15341194]

125 Zöröglu SS, Yürekli M, Meram I, Sögüt S, Tutkun H, Yetkin O, et al. Pathophysiological role of nitric oxide and adrenomedullin in autism. Cell Biochem Funct 2003; 21(1): 55-60. [DOI: 10.1002/ cbf.989]; [PMID: 12579522]

126 Darley-Usmar VM, Hogg N, O’Leary VJ, Wilson MT, Moncada $\mathrm{S}$. The simultaneous generation of superoxide and nitric oxide can initiate lipid peroxidation in human low density lipoproteins. Free Radic Res Commun 1992; 17(1): 9-20]; [PMID: 1332919]

127 Hogg N, Darley-Usmar VM, Graham A, Moncada S. Peroxynitrite and atherosclerosis. Biochem Soc Trans 1993; 21(2): 358-362. [DOI: 10.1042/bst0210358]; [PMID: 8359496]

128 Ziouzenkova O, Asatryan L, Akmal M, Tetta C, Wratten ML, Loseto-Wich G, et al. Oxidative cross-linking of ApoB100 and hemoglobin results in low density lipoprotein modification in blood. J Biol Chem 1999: 274(27): 18916-18924. [DOI: 10.1074/ jbc.274.27.18916]; [PMID: 10393389]

129 Lenz ML, Hughes H, Mitchell JR, Via DP, Guyton JR, Taylor $\mathrm{AA}$, et al. Lipid hydroperoxy and hydroxy derivatives in coppercatalyzed oxidation of low density lipoprotein. J Lipid Res 1990; 31(6): 1043-1050]; [PMID: 2373954]

130 Salonen JT, Yla-Herttuala S, Yamamoto R, Butler S, Korpela $\mathrm{H}$, Salonen $\mathrm{R}$, et al. Autoantibody against oxidised LDL and progression of carotid atherosclerosis. Lancet 1992; 339(8798): 883-887. [DOI: 10.1016/0140-6736(92)90926-T]; [PMID: 
1348295]

131 Ruef J, Peter K, Nordt TK, Rungs MS, Kübler W, Bode C. Oxidative stress and atherosclerosis: ist relationship to growth factors, thrombus formation and therapeutic approaches. Thromb Haemost 1999; 82 Suppl 1: 32-37]; [PMID: 10695483]

132 Moore SA, Stooker W, Lagrand WK, Van den Brule AJ, Niessen HW. Microorganisms in the aetiology of atherosclerosis. $J$ Clin Pathol 2000; 53(9): 647-654. [DOI: 10.1136/jcp.53.9.647. PMCID: 1731245]

133 Al-Kennany ER. Pathological study of the capability of Toxoplasma gondii to induce oxidative stress and initiation a primary lesion of atherosclerosis experimentally in broiler chickens. J Anim Vet Advances 2007; 6(8): 938-942. ISSN: 16805593]

134 Hassan SM, Al-Kennany ER, Al-Hafez HAK. Hydrogen peroxide induced atherosclerosis in chickens: effect of vitamin C. Iraqui $J$ Vet Sci 2000; 13: 249-270. ISSN: 1607-3894

135 Al-Kennany ER. Capability of Toxoplasma gondii to induce an oxidative stress and initiation of atherosclerotic lesions in cats experimentally infected. Iraqui $J$ Vet Sci 2006; 20: 165-176. ISSN: 1607-3894

136 Yang JF, Yue HP, Hou YY, Liu ZS, Rao HX, He YX, et al. Acute infection of Toxoplasma gondii affects the level of oxygen free radicals in serum and testes of mice. Zhongguo Ji Sheng Chong Xue Ji Sheng Chong Bing Za Zhi 2010; 28(5): 3364-3367. [PMID: 21351550]

137 Karaman U, Celik T, Kiran TR, Colak C, Daldal NU. Malondialdehyde, glutathione, and nitric oxide levels in Toxoplasma gondii seropositive patients. Korean J Parasitol 2008; 46(4): 293-295. [DOI: 10.3347/kjp.2008.46.4.293]; [PMID: 191227340]

138 Yazar S, Kilic E, Saraymen R, Sahin I. Serum malondialdehyde levels in Toxoplasma seropositive patients. Ann Saudi Med 2003; 23(6): 413-415]; [PMID: 16868383]

139 Elsheikha HM, El-Motayam MH, Abouel-Nour MF, Morsy AT. Oxidative stress and immune-suppression in Toxoplasma gondii positive blood donors: implications for safe blood transfusion. $J$ Egypt Soc Parasitol 2009; 39(2): 421-426.

140 Abousamira NK. Toxoplasma gondii antibodies and associated oxidative stress among asymptomatic blood donors. Ann Emerg Med 2008; 52: 138 (Research Forum Abstracts).

141 Foroutan-Rad M, Majidiani H, Dalvand A, Daryani A, Kooti W, Saki J, et al. Toxoplasmosis in blood donors: a systematic review and meta-analysis. Transfus Med Rev 2016; 30(3): 116-122. [DOI: 10.1016/j.tmrv.2016.03.002]; [PMID: 27145927]

142 Stommel EW, Cho E, Steide JA, Seguin R, Barchowsky A, Schwartzman JD, et al. Identification and role of thiols in Toxoplasma gondii egress. Exp Biol Med (Maywood) 2001; 226(3): 229-236]; [PMID: 11361042]

143 Jafari M, Salehi M, Shirbazou S, Abasian L, Talebi-Meymand F, Evaluation of gender-related differences in response to oxidative stress in Toxoplasma gondii positive serum. Ann Milit Health Res 2014; 12(2): 64-69.

144 Vegeto E, Pollio G, Ciana P, Maggi A. Estrogen blocks inducible nitric oxide synthase accumulation in LPS-activated microglia cells. Exp Gerontol 2000; 35(9-10): 1309-1316. [DOI: 10.1016/ S0531-5565(00)00161-3]; [PMID: 11113609]

145 Dincel GC, Atmaca HT. Role of oxidative stress in the pathophysiology of Toxoplasma gondii infection. Int $J$ Immunopathol Pharmacol 2016; 29(2): 226-240. [DOI: 10.1177/0394632016638668]; [PMID: 26966143]

146 Charron AJ, Sibley LD. Host cells: mobilizable lipid resources for the intracellular parasite Toxoplasma gondii. J Cell Sci 2002; 115(Pt 15): 3049-3059]; [PMID: 12118061]

147 Gupta N, Zahn MM, Coppens I, Joiner KA, Voelker DR. Selective disruption of phosphatidylcholine metabolism of the intracellular parasite Toxoplasma gondii arrests its growth. J Biol Chem 2005;
280(16): 16345-16353. [DOI: 10.1074/jbc.M501523200]; [PMID: 15708856]

148 Nishikawa Y, Ibrahim HM, Kameyama K, Shiga I, Hiasa J, Xuan $\mathrm{X}$. Host cholesterol synthesis contributes to growth of intracellular Toxoplasma gondii in macrophages. $J$ Vet Med 2011; 73(5): 633639. [DOI: 10.1292/jvms.10-0496]; [PMID: 21187676]

149 Coppens I, Sinai AP, Joiner KA. Toxoplasma gondii exploits host low-density lipoprotein receptor-mediated endocytosis for cholesterol acquisition. $J$ Cell Biol 2000; 149(1): 167-180. [DOI: 10.1083/jcb.149.1.167]; [PMID: 10747095]

150 Lange Y, Steck TL. The role of intracellular cholesterol transport in cholesterol homeostasis. Trends Cell Biol 1996; 6(6): 205-208. [DOI: 10.1016/0962-8924(96)20016-9]; [PMID: 15157456]

151 Liscum L, Munn NJ. Intracellular cholesterol transport. Biochim Biophys Acta 1999; 1438(1): 19-37. [DOI: 10.1016/S13881981(99)00043-8]; [PMID: 10216277]

152 Sakashita N, Miyazaki A, Chang CC, Chang TY, Kiyota E, Satoh $\mathrm{M}$, et al. Acyl-coenzym A: cholesterol acyltransferase 2 (ACAT2) is induced in monocyte-derived macrophages: in vivo and in vitro studies. Lab Invest 2003; 83(11): 1569-1581]; [PMID: 14615411]

153 Lige B, Sampels V, Coppens I. Characterization of a second sterolesterifying enzyme in Toxoplasma highlights the importance of cholesterol storage pathways for the parasite. Mol Microbiol 2013; 87(5): 951-967. [DOI: 10.1111/mmi.12142]; [PMID: 23374239]

154 Nishikawa Y, Quittnat F, Stedman TT, Voelker DR, Choi JY, Zahn $\mathrm{M}$, et al. Host cells lipids control cholesteryl ester synthesis and storage in intracellular Toxoplasma. Cell Microbiol 2005; 7(6): 849-867. [DOI: $10.1111 / \mathrm{j} .1462-5822.2005 .00518 x]$; [PMID: 15888087]

155 Cases S, Novak S, Zheng Y-W, Myers HM, Lear SR, Sande $\mathrm{E}$, et al. ACAT2, a second mammalian acyl-CoA: cholesterol acyltransferase. $J$ Biol Chem 1998; 273(41): 26755-26764. [DOI: 10.1074/jbc.273.41.26755]; [PMID: 9756919]

156 Quittnat F, Nishikawa Y, Stedman T, Voelker DL, Choi JY, Zahn $\mathrm{MM}$, et al. On the biogenesis of lipid bodies in ancient eukaryotes: synthesis of triacylglycerols by a Toxoplasma DGAT1-related enzyme. Mol Biochem Parasitol 2004; 138(1): 107-122. [DOI: 10.1016/j.molbiopara.2004.08.004]; [PMID: 15500922]

157 Sonda S, Ting LM, Novak S, Kim K, Maher JJ, Farese RV, et $a l$. Cholesterol esterification by host and parasite is essential for optimal proliferation of Toxoplasma gondii. J Biol Chem 2001; 276(37): 34434-34440. [DOI: 10.1074/jbc.M105025200]; [PMID: 11457847]

158 Cases S, Smith SJ, Zheng YW, Myers HM, Lear SR, Sande E, et $a l$. Identification of a gene encoding an acyl CoA: diacylglycerol acyltransferase, a key enzyme in triacylglycerol synthesis. Proc Natl Acad Sci USA 1998; 95(22): 13018-13023. [DOI: 10.1073/ pnas.95.22.13018]; [PMID: 9789033]

159 Lei L, Xiong Y, Chen J, Yang J-B, Wang Y, Yang XY, et al. TNF- $\alpha$ lpha stimulates the ACAT1 expression in differentiating monocytes to promote CE-laden cell formation. J Lipid Res 2009; 50(6): 1057-1067. [DO: 10.1194/jlr.M800484-JLR200]; [PMID: 19189937]

160 Habib A, Finn AV. The role of iron metabolism as a mediator of macrophage inflammation and lipid handling in atherosclesosis. Front Pharmacol 2014; 6: 195. [DOI: 10.3389/fphar.2014.00195]; [PMID: 25221612]

161 Ruan XZ, MooreheadJF, Tao JL, Ma KL, Wheeler DC, Powis SH, et al. Mechanism of dysregulation of low-density lipoprotein receptor expression in vascular smooth muscle cells by inflammatory cytokines. Arterioscl Thromb Vasc Biol 2006; 26(5): 1150-1155. [DOI: 10.1161/01.ATV.0000217957.93135.c2]; [PMID: 16543490]

162 DePalma RG, Hayes VW, Cafferata HT, Mohammadpour HA, Chow BK, Zacharski LR, et al. Cytokine signatures in atherosclerosis claudicants. J Surg Res 2003; 111(2): 215-221. [DOI: 10.1016/S0022-4804(03)00075-1]; [PMID: 12850465] 
163 Ridker PM. Inflammation, atherosclerosis, and cardiovascular risk: an epidemiologic view. Blood Coagul Fibrinolysis 1999; 10(Suppl 1): S9-S12]; [PMID: 10070810]

164 Prandota J. Unexplained liver damage, cryptogenic liver cirrhosis, and steatohepatitis may be caused by latent chronic toxoplasmosis. OA Hepatology 2014; 2(1): 2.

165 Prandota J. Gastrointestinal tract abnormalities in autism, inflammatory bowel disease and many other clinical entities may be due to T. gondii infection. Sci Rep 2012; 1: 4. [DOI: 10.4172/ scientificreports. 256

166 Dubey JP. Advances in the life cycle of Toxoplasma gondii. Int J Parasitol 1998; 28(7): 1019-1024. [DOI: 10.1016/S00207519(98)00023-X]; [PMID: 9724872]

167 Pacifico L, Cantisani V, Ricci P, Osborn JF, Schiavo E, Anania C, et al. Nonalcoholic fatty liver disease and carotid atherosclerosis in children. Pediatr Res 2008; 63(4): 423-427. [DOI: 10.1203/ PDR.0b013e318165b8e7]; [PMID: 18356751]

168 Alp H, Karaarslan S, Selver Eklioglu B, Atabek ME, Altun H, Baysal T. Association between nonalcoholic fatty liver disease and cardiovascular risk in obese children and adolescents. Can J Cardiol 2013; 29(9): 1118-1125. [DOI: 10.1016/ j.cjca.2012.07.846]; [PMID: 23040432]

169 Sookoian S, Pirola CJ. Non-alcoholic fatty liver disease is strongly associated with carotid atherosclerosis: a systematic review. $J$ Hepatol 2008; 49(4): 600-607. [DOI: 10.1016/j.jhep.2008.06.012]; [PMID: 18672311]

170 Fargion S, Porzio M, Fracanzani AL. Nonalcoholic fatty liver disease and vascular disease: state-of-the-art. World $J$ Gastroenterol 2014; 20(37): 13306-13324. [DOI: 10.3748/wjg. v20.i37.13306]; [PMID: 25309067]

171 Aronow WS. Nonalcoholic fatty liver disease is associated with coronary artery disease and subclinical atherosclerosis. Future Cardiol 2016; 12(4): 393-396. [DOI: 10.2217/fca-2016-0033]; [PMID: 27291253]

172 Boyer TD, Manns MP, Sanyal AJ. Zakim \& Boyer's Hepatology, A Textbook of Liver Disease. $6^{\text {th }}$ ed. USA: Saunders 2011; pp. 941968. ISBN-13: 978-1437708813

173 Schwimmer JB, Deutsch R, Kahen T, Lavine JT, Stanley C, Behling C. Prevalence of fatty liver in children and adolescents. Pediatrics 2006; 118(4): 1388-1393. [DOI: 10.1542/peds.20061212]; [PMID: 17015527]

174 Vajro P, Lenta S, Socha P, Dhawan A, McKiernan P, Baumann $\mathrm{U}$, et al. Diagnosis of nonalcoholic fatty liver disease in children and adolescents: position paper of the ESPGHAN Hepatology Committee 2012; 54(5): 700-713. [DOI: 10.1097/ MPG.0b013e518252a13f]; [PMID: 22395188]

175 Hartman J, Frishman WH. Inflammation and atherosclerosis: a review of the role of interleukin- 6 in the development of atherosclerosis and the potential for targeted drug therapy. Cardiol Rev 2014; 22(3): 147-151. [DOI: 10.1097/ CRD.0000000000000021]; [PMID: 24618929]

$176 \mathrm{Yu}$ XH, Fu YC, Zhang DW, Yin K, Tang CK. Foam cells in atherosclerosis. Clin Chim Acta 2013; 424: 245-252. [DOI: 10.1016/j.cca.2013.06.006]; [PMID: 23782937]

177 Chang C, Dong R, Miyazaki A, Sakashita N, Zhang Y, Liu J, et al. Human acyl-CoA : cholesterol acyltransferase (ACAT) and its potential as a target for pharmaceutical intervention against atherosclerosis. Acta Biochim Biophys Sin (Shanghai) 2006; 38(3): 151-156. [DOI: 10.1111/j.1745-7270.2006.00154.x]; [PMID: 16518538]

178 Sakai M, Kobori S, Miyazaki A, Horiuchi S. Macrophage proliferation in atherosclerosis. Curr Opin Lipidol 2000; 11(5): 50350-50359]; [PMID: 11048893]

179 Lei X, Fujiwara Y, Chang CC, Chang TY, Takeya M, Sakashita N. Association of ACAT1-positive vesicles with late endosomes/ lysosomes in cholesterol-rich human macrophages. $J$ Atheroscler Thromb 2010; 17(7): 740-750. [DOI: 10.551/jat.4416]; [PMID:
20523008]

180 Lusis AJ. Atherosclerosis. Nature 2000; 407(6801): 233-241. [DOI: 10.1038/35025203]; [PMID: 11001066

181 Chang TY, Chang CC, Lin S, Yu C, Li L, Myazaki A. Roles of acyl-coenzyme A: cholesterol acyltransferase-1 and -2. Curr Opin Lipidol 2001; 12(3): 289-296]; [PMID: 11353332]

182 Rudel LL, Lee RG, Cockman TL. Acyl coenzyme A: cholesterol acyltransferase types 1 and 2: structure and function in atherosclerosis. Curr Opin Lipidol 2001; 12(2): 121-127]; [PMID: 11264983]

183 Santamarina-Fojo S, Lambert G, Hoeg JM, Brewer HB Jr. Lecithin-cholesterol acyltransferase: role in lipoprotein metabolism, reverse cholesterol transport and atherosclerosis. Curr Opin Lipidol 2000; 11(3): 267-275. [PMID: 10882342]

184 Rudel LL, Shelness GS. Cholesterol esters and atherosclerosis a game of ACAT and mouse. Nat Med 2000; 6(12): 1313-1314. [DOI: 10.1038/82110]; [PMID: 11100106]

185 Chang TY, Chang CCY, Cheng D. Acyl-coenzyme A: cholesterol acyl-transferase. Annu Rev Biochem 1997; 66: 613-638. [DOI: 10.1146/annurev.biochem.66.1.613]; [PMID: 9242919]

186 Tabas I. Consequences of cellular cholesterol accumulation: basic concepts and physiological implications. J Clin Invest 2002; 110(7): 905-911. [DOI: 10.1172/JCI16452]; [PMID: 12370266]

187 Warner GJ, Stoudt G, Bamberger M, Johnson WJ, Rothblat GH. Cell toxicity induced by inhibition of acyl coenzyme A: cholesterol acyltransferase and accumulation of unesterfied cholesterol. J Biol Chem 1995; 270(11): 5772-5778. [DOI: 10.1074/jbc.270.11.5772]; [PMID: 7890706]

188 Suguro T, Watanabe T, Kanome T, Kodate S, Hirano T, Miyazaki A, et al. Serotonin acts as an up-regulator of acyl-coenzyme A: cholesterol acyltransferase-1 in human monocyte-macrophages. Atherosclerosis 2006; 186(2): 275-281. [DOI: 10.1016/ j.atherosclerosis.2005.08.007]; [PMID: 16157345]

189 Yin YW, Liao SQ, Zhang MJ, Liu Y, Li BH, Zhou Y, et al. TLR4-mediated inflammation promotes foam cell formation of vascular smooth muscle cell by upregulating ACAT1 expression. Cell Death Dis 2014; 5: e1574. [DOI: 10.1038/cddis.2014.535]; [PMID: 25522268]

190 Seo T, Oelkers PM, Giattina MR, Worgall TS, Sturley SL, Deckelbaum RJ. Differential modulation of ACAT1 and ACAT2 transcription and activity by long chain free fatty acids in cultured cells. Biochemistry 2001; 40(15): 4756-4762. [DOI: 10.1021/ bi0022947]; [PMID: 11294643]

191 Miyazaki A, Sakashita N, Lee O, Takahashi H, Horiuchi S, Hakamata $\mathrm{H}$, et al. Expression of ACAT-1 protein in human atherosclerotic lesions and cultured human monocytemacrophages. Arterioscler Thromb Vasc Biol 1998; 18(10): 15681574. [DOI: 10.1161/01.ATV.18.101568]; [PMID: 9763528]

192 Cheng D, Chang CCY, Qu X, Chang TY. Activation of acylcoenzyme A: cholesterol acyltransferase by cholesterol or by oxysterol in a cell free system. J Biol Chem 1995; 270(2): 685 695. [DOI: 10.1074/jbc.270.2.685]; [PMID: 7822296]

193 Dove DE, Su YR, Swift LL, Linton MF, Fazio S. ACAT1 deficiency increases cholesterol synthesis in mouse peritoneal macrophages. Atherosclerosis 2006; 186(2): 267-274. [DOI: 10.1016/j.atherosclerosis.2005.08.005]; [PMID: 16144700]

194 Yang L, Yang JB, Chen J, Yu GY, Zhou P, Lei L, et al. Enhancement of human ACAT1 gene expression to promote the macrophage-derived foam cell formation by dexamethasone. Cell Res 2004; 14(4): 315-323. [DOI: 10.1038/sj.cr.7290231]; [PMID: $15353128]$

195 Rong JX, Kusunoki J, Oelkers P, Sturley SL, Fisher EA. Acylcoenzym A (CoA): cholesterol acyltransferase inhibition in rat and human aortic smooth muscle cells is nontoxic and retards foam cell formation. Arterioscler Thromb Vasc Biol 2005; 25(1): 122-127. [DOI: 10.1161/01.ATV.0000148202.49842.3b]; [PMID: 15499046] 
196 Yang K, Zhang XJ, Cao LJ, Liu XH, Liu ZH, Wang XQ, et al. Toll-like receptor 4 mediates inflammatory cytokine secretion in smooth muscle cells induced by oxidized low-density lipoprotein. PLoS One 2014; 9(4): e95935. [DOI: 10.1371/journal. pone.0095935]; [PMID: 24755612]

197 Zhang LL, Gao CY, Fang CQ, Wang YJ, Gao D, Yao GE, et al. PPARg attenuates intimal hyperplasia by inhibiting TLR4mediated inflammation in vascular smooth muscle cells. Cardiovasc Res 2011; 92(3): 484-493. [DOI: 10.1093/cvr/cvr238]; [PMID: 21880694]

198 Sakashita N, Miyazaki A, Takeya M, Horiuchi S, Chang CC, Chang TY, et al. Localization of human acyl-coenzym A: cholesterol acyltransferase-1 (ACAT-1) in macrophages and in various tissues. Am J Pathol 2000; 156(1): 227-236. [DOI: 10.1016/S0002-9440(10)64723-2]; [PMID: 10623671]

199 Uelmen PJ, Oka K, Sullivan M, Chang CCY, Chang TY, Chan L. Tissue-specific expression and cholesterol regulation of acylcoenzyme A: cholesterol acyltransferase (ACAT) in mice: molecular cloning of ACAT in vitro and in vivo. J Biol Chem 1995; 270(44): 26192-26201. [DOI: 10.1074/jbc.270.44.26192]; [PMID: 7592824]

200 Pape ME, Schultz PA, Rea TJ, DeMattos RB, Kieft K, Bisgaier $\mathrm{CL}$, et al. Tissue specific changes in acyl-CoA: cholesterol acyltransferase (ACAT) mRNA levels in rabbits. J Lipid Res 1995; 36(4): 823-838]; [PMID: 7616126]

201 Cheng W, Kvilekval KV, Abumrad NA. Dexamethasone enhances accumulation of cholesteryl esters by human macrophages. Am J Physiol 1995; 269(4 Pt 1): E642-E648. [PMID: 7485476]

202 Yang JB, Duan ZJ, Yao W, Lee L, Yang L, Yang XY, et al. Synergistic transcriptional activation of human acyl-coenzym A: cholesterol acyltransferase-1 gene by interferon-gamma and all-trans-retinoic acid THP-1 cells. J Biol Chem 2001; 276(24): 20989-20998. [DOI: 10.1074/jbc.M011488200]; [PMID: 1139977]

203 Maung K, Miyazaki A, Nomiyama H, Chang CC, Chang TY, Horiuchi S. Induction of acyl-coenzyme A: cholesterol acyltransferase- 1 by 1,25-dihydroxyvitamin $\mathrm{D}(3)$ or 9-cis-retinoic acid in undifferentiated THP-1 cells. J Lipid Res 2001; 42(2): 181187]; [PMID: 11181746]

204 Seino K, Yamauchi T, Shikata K, Kobayashi S, Nagai M, Taniguchi $\mathrm{M}$, et al. Prevention of acute and chronic allograft rejection by a novel retinoic acid receptor-alpha-selective agonist. Int Immunol 2004; 16(5): 665-673. [DOI: 10.1093/intimm/ dxh066]; [PMID: 15096490]

205 Ihara F, Nishikawa Y. Synthetic retinoid Am80 controls growth of intracellular Toxoplasma by inhibiting acquisition and synthesis of cholesterol in macrophages. J Protozool Res 2014; 24: 1-10. NAID: 120005606850

206 Takeda N, Manabe I, Shindo T, Iwata H, Iimuro S, Kagechika $\mathrm{H}$, et al. Synthetic retinoid Am80 reduces scavenger receptor expression and atherosclerosis in mice by inhibiting IL-6. Arterioscler Thromb Vasc Biol 2006; 26(5): 1177-1183. [DOI: 10.1161/01.ATV.0000214296.94849.1c]; [PMID: 16484594]

207 Ge J, Zhai W, Cheng B, He P, Qi B, Lu H, et al. Insulin induces human acyl-coenzyme A: cholesterol acyltransferase 1 gene expression via MAP kinases and CCAAT/enhancer-binding protein alpha. $J$ Cell Biochem 2013; 114(9): 2188-2198. [DOI: 10.1002/jcb.24568]; [PMID: 23564383]

208 Hongo S, Watanabe T, Arita S, Kanome T, Kageyama H, et al. Leptin modulates ACAT1 expression and cholesterol efflux from human macrophages. Am J Physiol Endocrinol Metab 2009 ; 297(2): E474-E482. doi : 10.1152/ajpendo.90369.2008]; [PMID: 19625677]

209 Hori M, Miyazaki A, Tamagawa H, Satoh M, Furukawa K, Hakamata $\mathrm{H}$, et al. Up-regulation of acyl-coenzyme A: cholesterol acyltransferase-1 by transforming growth factor-b1 during differentiation of human monocytes into macrophages. Biochem
Biophys Res Commun 2004; 320(2): 501-505. [DOI: 10.1016 j.bbrc.2004.05.190]; [PMID: 15219857]

210 Nagineni CN, Detrick B, Hooks JJ. Transforming growth factor-beta expression in human retinal pigment epithelial cells is enhanced by Toxoplasma gondii: A possible role in the immunopathogenesis of retinochoroiditis. Clin Exp Immunol 2002; 128(2): 372-378. [DOI: 10.1046/j.1365-2249.2002.01815. x]; [PMID: 11985530]

211 Campbell LA, Kuo C-C, Graystone JT. Chlamydia pneumoniae and cardiovascular disease. Emerg Infect Dis 1998; 4(4): 571-579. [DOI: 10.3201/eid0404.980407]; [PMID: 9866733]

212 Rupp J, Pfleiderer L, Jugert C, Moeller S, Klinger M, Dalhoff K, et al. Chlamydia pneumoniae hides inside apoptotic neutrophils to silently infect and propagate in macrophages. PLOS ONE 2009; 4(6): e6020. [DOI: 10.1371/journal.pone.0006020]; [PMID: 19547701]

213 He P, Mei C, Cheng B, Liu W, Wang Y, Wan J. Chlamydia pneumoniae induces macrophage-derived foam cell formation by up-regulating acyl-coenzyme A: cholesterol acyltransferase 1. Microbes Infect 2009; 11(2): 157-163. [DOI: 10.1016/ j.micinf.2008.11.001]; [PMID: 19049899]

214 Liu W, He P, Cheng B, Mei CL, Wang YF, Wan JJ. Chlamydia pneumoniae disturbs cholesterol homeostasis in human THP-1 macrophages via JNK-PPARgamma dependent signal transduction pathways. Microbes Infect 2010; 12(14-15): 1226-1235. [DOI: 10.1016/j.micinf.2010.09.004]; [PMID: 20870032]

215 Khan S, Rahman HNA, Okamoto T, Matsunaga T, Fujiwara Y, Sawa $\mathrm{T}$, et al. Promotion of atherosclerosis by Helicobacter cinaedi infection that involves macrophage-driven proinflammatory responses. Sci Rep 2013; 4: 4680. [DOI: 10.1038/srep04680]; [PMID: 24732347]

216 Zhao ZZ, Wang Z, Li GH, Wang R, Tan JM, Cao X, et al. Hydrogen sulfide inhibits macrophage-derived foam cell formation. Exp Biol Med (Maywood) 2011; 236(2) : 169-176. [DOI: 10.1258/ebm.2010.010308]; [PMID: 21321313]

217 Wang Y, Zhao X, Jin H, Wei H, Li W, Bu D, et al. Role of hydrogen sulfide in the development of atherosclerotic lesions in apolipoprotein E knockout mice. Arterioscler Thromb Vasc Biol 2009; 29(2): 173-179. [DOI: 10.1161/ATVBAHA.108.179333]; [PMID: 18988885]

218 Sun Y, Tang CS, Du JB, Jin HF. Hydrogen sulfide and vascular relaxation. Chin Med J (Engl) 2011; 124(22): 3816-3819]; [PMID: 22340247]

219 Giuliani D, Ottani A, Zaffe D, Galantucci M, Strinati F, Lodi R, et al. Hydrogen sulfide slows down progression of experimental Alzheimer's disease by targeting multiple pathophysiological mechanisms. Neurobiol Learn Mem 2013; 104: 82-91. [DOI: 10.1016/j.nlm.2013.05.006]; [PMID: 23726868]

220 Yu XH, Cui LB, Wu K, Zheng XL, Cayabyab FS, Chen ZW, et al. Hydrogen sulfide as a potent cardiovascular protective agent. Clin Chim Acta 2014; 437: 7 8-87. [DOI: j.cca.2014.07.012]; [PMID: 25058799]

221 Lathe R, Sapronova A, Kotelevtsev Y. Atherosclerosis and Alzheimer - diseases with a common cause ?. Inflammation, oxysterols, vasculature. BMC Geriatrics 2014; 14: 36. [DOI: 10.1186/1471-2318-14-36]; [PMID: 24656052]

222 Prandota J. Metabolic, immune, epigenetic, endocrine and phenotypic abnormalities found in individuals with autism spectrum disorders, Down syndrome and Alzheimer disease may be caused by congenital and/or acquired chronic cerebral toxoplasmosis. Res Autism Spectr Disord 2011; 5(1): 14-59. [DOI: 10.1016/j.rasd.2010.03.009]

223 Prandota J. Possible link between T. gondii and the anosmia associated with neurodegenerative diseases. $\mathrm{Am} J$ Alzheimer's Dis Other Dement 2014; 29(3): 205-214. [DOI: 10.1177/1533317513517049]; [PMID: 24413543]

224 Rudel LL, Lee RG, Parini P. ACAT2 is a target for treatment of 
coronary heart disease associated with hypercholesterolemia. Arterioscler Thromb Vasc Biol 2005; 25(6): 1112-1118. [DOI: 10.1161/01.ATV.0000166548.65753.1e]; [PMID: 15831806]

225 Chang TY, Li BL, Chang CC, Urano Y. Acyl-coenzym A. cholesterol acyltransferases. Am J Physiol Endocrinol Metab 2009; 297(1): E1-E9. [DOI: 10.1152/ajpendo.90926.2008]; [PMID: 19141679]

226 Parini P, Davis M, Lada AT, Erickson SK, Wright TL, Gustafsson $\mathrm{U}$, et al. ACAT2 is localized to hepatocytes and is the major cholesterol-esterifying enzyme in human liver. Circulation 2004; 110(14): 2017-223. [DOI: 10.1161/01. CIR.0000143163.76212.0B]; [PMID: 15451793]

227 Oelkers P, Behari A, Cromley D, Billheimer JT, Sturley SL. Characterization of two human genes encoding acyl coenzyme A: cholesterol acyltransferase-related enzymes. J Biol Chem 1998; 273(41): 26765-26771. [DOI: 10.1074/jbc.273.41.26765]; [PMID: 9756920]

228 Buhman KK, Accad M, Novak S, Choi RS, Wong JS, Hamilton $\mathrm{RL}$, et al. Resistance to diet-induced hypercholesterolemia and gallstone formation in ACAT2-deficient mice. Nat Med 2000; 6(12): 1341-1347. [DOI: 10.1038/82153]; [PMID: 11100118]

229 Klein RL, Rudel LL. Cholesterol absorption and transport in thoracic duct lymph lipoproteins of nonhuman primates. Effect of dietary cholesterol level. J Lipid Res 1983; 24(4): 343-356]; [PMID: 68554146]

230 Williams DL, Connelly MA, Temel RE, Swarnakar S, Philips MC, de la Llera-Moya M, et al. Scavenger receptor BI and cholesterol trafficking. Curr Opin Lipidol 1999; 10(4): 329-339. [PMID: 10482136]

231 Huggins KW, Burleson ER, Sawyer JK, Kelly K, Rudel LL. Determination of the tissue sites responsible for the catabolism of large high density lipoprotein in the African green monkey. J Lipid Res 2000: 41(3): 384-394]; [PMID: 10706586]

232 Lee RG, Kelley KL, Sawyer JK, Farese RV Jr, Parks JS, Rudel LL. Plasma cholesterol esters provided by lectin: cholesterol acylatransferase and acyl-coenzym A: cholesterol acyltransferase 2 have opposite atherosclerotic potential. Circ Res 2004; 95(10): 998-1004. [DOI: 10.1161/01.RES.0000147558.1555467]; [PMID: 15486318]

233 Lee RG, Shah R, Sawyer JK, Hamilton RL, Parks JS, Rudel LL. ACAT2 contributes cholesteryl esters to newly secreted VLDL, whereas LCAT adds cholesteryl ester to LDL in mice. $J$ Lipid Res 2005; 46(6): 1205-1212. [DOI: 10.1194/jlr.M500018-JLR200]; [PMID: 15805543]

234 Temel RE, Hou L, Rudel LL, Selness GS. ACAT2 stimulates cholesteryl ester secretion in apoB-containing lipoproteins. $J$ Lipid Res 2007; 48(7): 1618-1627. [DOI: 10.1194/jlr.M700109JLR200]; [PMID: 17438337]

235 Rudel LL, Haines J, Sawyer JK, Shah R, Wilson MS, Carr TP. Hepatic origin of cholesteryl oleate in coronary artery atherosclerosis in African green monkeys. Enrichment by dietary monounsaturated fat. $J$ Clin Invest 1997; 100(1): 74-83. [DOI: 10.1172/JCI119524]; [PMID: 9202059]

236 Furbee JW, Sawyer JK, Parks JS. Lecithin: cholesterol acyltransferase deficiency increases atherosclerosis in the low density lipoprotein receptor and apolipoprotein E knockout mice. J Biol Chem 2002; 277(5): 3511-3519. [DOI: 10.1074/jbc. M109883200]; [PMID: 11719520]

237 Glomset JA. The plasma lecithins: cholesterol acyltransferase reaction. J Lipid Res 1968; 9(2): 155-167]; [PMID: 4868699]

238 Kirkeby K, Ingvaldsen P, Bjerkedal I. Fatty acid composition of serum lipids in men with myocardial infarction. Acta Med Scand 1972; 192(6): 513-519]; [PMID: 4640696]

239 Kingsbury KJ, Brett C, Stovold R, Chapman A, Anderson J, Morgan DM. Abnormal fatty acid composition and human atherosclerosis. Postgrad Med J 1974; 50(585): 425-440]; [PMID: 4469034]
240 Degirolamo C, Shelness GS, Rudel LL. LDL cholesterol oleate as a predictor for atherosclerosis: evidence from human and animals studies on dietary fat. J Lipid Res 2009; 50(Suppl): S434-S439. [DOI: 10.1194/jlr.R800076-JLR200]; [PMID: 19029117]

241 Tall AR, Small DM, Atkinson D, Rudel LL. Studies on the structure of low density lipoproteins isolated from Macaca fascicularis fed an atherogenic diet. $J$ Clin Invest 1978; 62(6): 1354-1363. [DOI: 10.1172/JCI109256]; [PMID: 219029]

242 Lada AT, Rudel LL, St Clair RW. Effects of LDL enriched with different dietary fatty acids on cholesteryl ester accumulation and turnover in THP-1 macrophages. J Lipid Res 2003; 44(4): 770779. [DOI: 10.1194/jlr.M20043-JLR200]; [PMID: 12562836]

243 Spector AA, Haynes WG. LDL cholesteryl oleate. A biomarker for atherosclerosis ?. Arterioscler Thromb Vasc Biol 2007; 27(6): 1228-1230. [DOI: 10.1161/ATVBAHA.107.147082]; [PMID: 17522395]

244 Avramoglu RK, Cianflone K, Sniderman AD. Role of the neutral lipoid accessible pool in the regulation of secretion of apoB-100 lipoprotein particles by HepG2 cells. J Lipid Res 1995; 36(12): 2513-2528]; [PMID: 88447478]

245 Meiner VL, Cases S, Myers HM, Sande ER, Bellosta S, et al. Disruption of the acyl-CoA: cholesterol acyltransferase gene in mice: Evidence suggesting multiple cholesterol esterification enzymes in mammals. Proc Natl Acad Sci USA 1996; 93(24): 14041-14046]; [PMID: 8943057]

246 Liang JJ, Oelkers P, Guo C, Chu PC, Dixon JL, Ginsberg HN, et al. Overexpression of human diacylglycerol acyltransferase 1, acyl-CoA: cholesterol acyltransferase 1 or acyl-CoA: cholesterol acyltransferase 2 stimulates secretion of apolipoprotein B-containing lipoproteins in McA-RH7777 cells. $J$ Biol Chem 2004; 279(43): 44938-44944. [DOI: 10.1074/jbc.M408507200]; [PMID: 15308631]

247 Rudel LL, Parks JS, Sawyer JK. Compared with dietary monounsaturated and saturated fat, polyunsaturated fat protects African green monkeys from coronary artery atherosclerosis. Arterioscler Thromb Vasc Biol 1995; 15(12): 2101-2110. [DOI: 10.1161/01.ATV.15.12. 2101]; [PMID: 7489230]

248 Miller CD, Thomas MJ, Hiestand B, Samuel MP, Wilson MD, Sawyer J, et al. Cholesteryl esters associated with ACAT2 predict coronary artery disease in patients with symptoms of acute coronary syndrome. Acad Emerg Med 2012; 19(6): 673-682. [DOI: $10.1111 / \mathrm{j} .1553-2712.2012 .01378 . x]$; [PMID: 22687182]

249 Wagner JD. Effects of sex steroid treatment on the cardiovascular system. Infert Reprod Med Clinics North Am 2001; 12: 511-533.

250 Kavanagh K, Davis MA, Zhang L, Wilson MD, Register TC, Adams MR, et al. Estrogen decreases atherosclerosis in part by reducing hepatic acyl-CoA: cholesterol acyltransferase 2 (ACAT2) in monkeys. Arterioscler Thromb Vasc Biol 2009; 29(10): 1471-1477. [DOI: 10.1161/ATVBAHA.109.191825]; [PMID: 19759374]

251 Parini P, Jiang ZY, Einarsson C, Eggersten G, Zhang SD, Rudel LL, et al. ACAT2 and human hepatic cholesterol metabolism: identification of important gender-related differences in normolipidemic, non-obese Chinese patients. Atherosclerosis 2009; 207(1): 266-271. [DOI: 10.1016 / j.atherosclerosis.2009.04.010]; [PMID: 19467657

252 Saba E, Jeon BR, Jeong DH, Lee K, Goo YK, Kim SH, et al. Black ginseng extract ameliorates hypercholesterolemia in rats. $J$ Ginseng Res 2016; 40(2): 160-168. [DOI: 10.1016/ j.jgr.2015.07.003]; [PMID: 27158237]

253 Van de Garde MDB, Martinez FO, Melgert BN, Hylkema MN, Jonkers RE, et al. Chronic exposure to glucocorticoids shapes gene expression and modulates innate and adaptive activation pathways in macrophages with distinct changes in leukocyte attraction. J Immunol 2014; 192(3): 1196-1208. [DOI: 10.4049/ jimmunol.1302138]; [PMID: 24395918]

254 Milenkovic D, Deval C, Dubray C, Mazur A, Morand C. 
Hesperidin displays relevant role in the nutrigenomic effect of orange huice on blood leukocytes in human volunteers: a randomized controlled cross-over study. PLOS ONE 2011; 6: e6669. [DOI: 10.1371/journal.pone.0026669]; [PMID: 22110589]

255 Nahmias Y, Goldwasser J, Casali M, van Poll D, Wakita T, Chung RT, et al. Apolipoprotein B-dependent hepatitis C virus secretion is inhibited by the grapefruit flavonoid naringenin. Hepatology 2008; 47(5): 1437-1445. [DOI: 10.1002/hep.22197]; [PMID: 18393287]

256 Zhao JF, Ching LC, Huang YC, Chen CY, Chiang AN, Kou YR, et al. Molecular mechanism of curcumin on the suppression of cholesterol accumulation in macrophage foam cells and atherosclerosis. Mol Nutr Food Res 2012; 56(5): 691-701. [DOI: 10.1002/mnfr.201100735]; [PMID: 22648616]

257 Shin SK, Ha TY, McGregor RA, Choi MS. Long-term curcumin administration protects against atherosclerosis via hepatic regulation of lipoprotein cholesterol metabolism. Mol Nutr Food Res 2011; 55(12): 1829-1840. [DOI: 10.1002/mnfr.201100440]; [PMID: 22058071]

258 Lu KY, Ching LC, Su KH, Yu YB, Kou YR, Hsiao SH, et al. Erythropoietin suppresses the formation of macophage foam cells. Role of liver X receptor a. Circulation 2010; 121: 18281837. [DOI: 10.1161/CIRCULATIONAHA.109.876839]; [PMID: 20385932]

259 Singla S, Sachdeva R, Mehta JL. Cannabinoids and atherosclerotic coronary heart disease. Clin Cardiol 2012; 35(6): 329-335. [DOI: 10.1002/clc.21962]; [PMID: 22278660]

260 Netherland C, Thewke DP. Rimonabant is a dual inhibitor of acyl CoA: Cholesterol acyltransferases 1 and 2. Biochem Biophys Res Commun 2010; 398(4): 671-676. [DOI: 10.1016/ j.bbrc.2010.06.134]; [PMID: 20609460]

261 Libby P, Ridker PM, Maseri A. Inflammation and atherosclerosis. Circulation 2002; 105(5): 1135-1143. [DOI: 10.1161/ hc0902.104353]; [PMID: 11877368]

262 Medzhitov R. Toll-like receptors and innate immunity. Nat Rev Immunol 2001; 1(2): 135-145. [DOI: 10.1038/35100529]; [PMID: 11905821]

263 Edfeldt K, Swedenborg J, Hansson G, Yan Z. Expression of Toll-like receptors in human atherosclerotic lesions. A possible pathway for plaque activation. Circulation 2002; 105(10): 11581161]; [PMID: 11889007]

264 Shinohara M, Hirata K, Yamashita T, Takaya T, Sasaki N, Shiraki $\mathrm{R}$, et al. Local overexpression of toll-like receptors at the vessel wall induces atherosclerotic lesion formation. Synergism of TLR2 and TLR4. Arterioscler Thromb Vasc Biol 2007; 27(11): 2384-2391. [DOI: 10.1161/ATVBAHA.106.139253]; [PMID: 17872459]

265 Poltorak A, He X, Smirnova I, Liu MY, Van Huffel C, Du X, et al. Defective LPS signaling In $\mathrm{C} 3 \mathrm{H} / \mathrm{HeJ}$ and $\mathrm{C} 57 \mathrm{BL} / 10 \mathrm{ScCr}$ mice: mutations In Tlr4 gene. Science 1998; 282(5396): 2085-2088. [DOI: 10.1126/science.282.5396.2085]; [PMID: 9851930]

266 Yarovinsky F. Toll-like receptors and their role in host resistance to Toxoplasma gondii. Immunol Letters 2008; 119(1-2): 17-21. [DOI: 10.1016/j.imlet.2008.05.007]; [PMID: 18617274]

267 Denkers EY. Toll-like receptor initiated host defense against Toxoplasma gondii. J Biomed Biotech 2010; 2010: 737125. [DOI: 10.1155/2010/737125]; [PMID: 19911079]

268 Björkbacka H, Kunjathoor VV, Moore KJ, Koehn S, Ordija CM, Lee MA, et al. Reduced atherosclerosis in MyD88-null mice links elevated serum cholesterol levels to activation of innate immunity signaling pathways. Nat Med 2004; 10(4): 416-4121. [DOI: 10.1038/nm1008]; [PMID: 15034566]

269 Michelsen KS, Wong MH, Shah PK, Zhang W, Yano J, Doherty $\mathrm{TM}$, et al. Lack of Toll-like receptor-4 or myeloid differentiation factor 88 reduces atherosclerosis and alters plaque phenotype in mice deficient in apolipoprotein E. Proc Natl Acad Sci USA 2004; 101(29): 10679-10684. [DOI: 10.1073/pnas.0403249101]; [PMID:
$15249654]$

270 Xi D, Zhao J, Lai W, Guo Z. Systemic analysis of the molecular mechanism underlying atherosclerosis using a text mining approach. Human Genomics 2016; 10(1): 14. [DOI: 10.1186/ s40246-016-0075-1]; [PMID: 27251057]

271 Tobias PS, Curtis LK. Toll-like receptors in atherosclerosis. Biochem Soc Trans 2007; 35(Pt 6): 1453-1455. [DOI: 10.1042/ BST0351453]; [PMID: 18031244]

272 Wasserman SM, Topper JN. Adaptation of the endothelium to fluid flow: in vitro analyses of gene expression and in vivo implications. Vasc Med 2004; 9(1): 35-45. [DOI: 10.1191/1358863x04vm521ra]; [PMID: 15230487]

273 Hodgkinson CP, Ye S. Toll-like receptors, their ligands, and atherosclerosis. Sci World J 2011; 11: 437-453. [DOI: 10.1100/ tsw.2011.36]; [PMID: 21336459]

274 Kodaira Y, Nair SK, Wrenshall LE, Gilboa E, Platt JL. Phenotypic and functional maturation of dendritic cell mediated by heparan sulfate. J Immunol 2000; 165(3): 1599-1604]; [PMID: 109033769]

275 Johnson GB, Brunn GJ, Kodaira Y, Platt JL. Receptor-mediated monitoring of tissue well-being via detection of soluble heparan sulfate by Toll-like receptor 4. J Immunol 2002; 168(10): 5233 5239. [DOI: 10.4049/jimmunol.168.10.5233]; [PMID: 11994480]

276 Zhang FX, Kirschning CJ, Manicelli R, Xu XP, Jin Y, Faure $\mathrm{E}$, et al. Bacterial lipopolysaccharide activates nuclear factor$\mathrm{kB}$ through IL-1 signaling mediators in cultured human dermal endothelial cells and mononuclear phagocytes. J Biol Chem 1999; 274(12): 7611-7614. [DOI: 10.1074/jbc.274.12.7611]; [PMID: 10075645

277 Rock FL, Hardiman G, Timans JC, Kastelein RA, Bazan JF. A family of human receptors structurally related to Drosophila Toll. Proc Natl Acad Sci USA 1998; 95(2): 588-593. [PMID: 9435236]

278 Underhill DM, Ozinsky A, Hajjar AM, Stevens A, Wilson $\mathrm{CB}$, Bassetti $\mathrm{M}$, et al. The Toll-like receptor 2 is recruited to macrophage phagosome and discriminates between pathogens. Nature 1999; 401(6755): 811-815. [DOI: 10.1038/44605]; [PMID: 10548109]

279 Medzhitov R, Preston-Hurlburt P, Janeway CA Jr. A human homologue of the Dropsophila Toll protein signals activation of adaptive immunity. Nature 1997; 388(6640): 394-397. [DOI: 10.1038/41131]; [PMID: 9237759]

$280 \mathrm{Xu}$ XH, Shah PK, Faure EF, Equils O, Thomas L, Fishbein MC, et al. Toll-like receptor-4 is expressed by macrophages in murine and human lipid-rich atherosclerotic plaques and upregulated by oxidized LDL. Circulation 2001; 104(25): 3103-3108. [DOI: 1161/hc5001.100631]; [PMID: 11748108]

281 Hoshino K, Takeuchi O, Kawai T, Sanjo H, Ogawa T, Takeda Y, et al. Toll-like receptor 4 (TLR4)-deficient mice are hyporesponsive to lipopolysaccharide: evidence for TRL4 as the Lps gene product. J Immunol 1999; 162(7): 3749-3752]; [PMID: 10201887]

282 Takeuchi O, Hoshino T, Akira S. TLR2-deficient and MyD88deficient mice are highly susceptible to Staphylococcus aureus infection. J Immunol 2000; 165(10): 5392-5396. [DOI: 10.4049/ jimmunol.165.10.5392]; [PMID: 11067888]

283 Takeda K, Kaisho T, Akira S. Toll-like receptors. Annu Rev Immunol 2003 ; 21: 335-376. [DOI: 10.1146/annurev. immunol.212.120601.141126]; [PMID: 12524386]

284 Berliner JA, Navab M, Fogelman AM, Frank JS, Demer LL, Edwards PA, et al. Atherosclerosis: basic mechanisms: oxidation, inflammation, and genetics. Circulation 1995; 91(9): 2488-2496. [DOI: 10.1161/01.CIR.91.9.2488]; [PMID: 7729036]

285 Brand K, Page S, Walli AK, Neumeier D, Baeuerle PA. Role of nuclear factor-kB in atherogenesis. Exp Physiol 1997; 82(2): 297-304. [DOI: 10.1113/expphysiol.1997.sp004025]; [PMID: 9129944]

286 Hertz C, Kiertscher G, Godowski P, Bouis DA, Norgard MV, Roth MD, et al. Microbial lipopeptides stimulate dendritic cell maturation via Toll-like receptor 2. J Immunol 2001; 166(4): 2444- 
2450. [DOI: 10.4049/jimmunol.166.4.2444]; [PMID: 11160304]

287 Jimenez R, Belcher E, Sriskandan S, Lucas R, McMaster S, Vojnovic I, et al. Role of Toll-like receptors 2 and 4 in the induction of cyclooxygenase-2 in vascular smooth muscle. Proc Natl Acad Sci USA 2005; 102(12): 4637-4642. [DOI: 10.1073/ pnas.0407655101]; [PMID: 15755814]

288 Otsui K, Inoue N, Kobayashi S, Shiraki R, Honjo T, Takahashi $\mathrm{M}$, et al. Enhanced expression of TLR4 in smooth muscle cells in human atherosclerotic coronary arteries. Heart Vessels 2007; 22(6): 416-422. [DOI: 10.1007/s00380-007-1001-1]; [PMID: 18044001]

289 Kol A, Libby P. Molecular mediators of arterial inflammation: a role for microbial products? Amer Heart $J$ 1999; 138(5 Pt 2): S450-S452]; [PMID: 10539846]

290 Ross R. The pathogenesis of atherosclerosis: a perspective for the 1990s. Nature 1993; 362(6423): 80180-80189. [DOI: 10.1038/362801a0]; [PMID: 8479518]

291 Wick G, Kleindienst R, Schett G, Amberger A, Xu Q. Role of heat shock protein $65 / 60$ in the pathogenesis of atherosclerosis. Int Arch Allergy Immunol 1995; 107(1-3): 130-131]; [PMID: 7542057]

292 Xu Q, Kleindienst R, Waitz W, Dietrich H, Wick G. Increased expression of heat shock protein 65 coincides with a population of infiltrating $\mathrm{T}$ lymphocytes in atherosclerotic lesions of rabbit specifically responding to heat shock protein 65. J Clin Invest 1993; 91(6): 2693-2702. [DOI: 10.1172/JCI116508]; [PMID: $8514876]$

293 Hammerer-Lercher A, Mair J, Bonatti J, Watzka SB, Puschendorf B, Dimhofer S. Hypoxia induces heat shock protein expression in human coronary artery bypass grafts. Cardiovasc Res 2001; 50(1): 115-124. [DOI: 10.1016/S0008-6363(01)00198-5]; [PMID: 11282084]

294 Berberian PA, Myers W, Tytell M, Challa V, Bond MG. Immunohistochemical localization of heat shock protein-70 in normal-appearing and atherosclerotic specimens of human arteries. Am J Pathol 1990; 136(1): 71-80]; [PMID: 2297051]

295 Johnson AD, Berberian PA, Tytell M, Bond MG. Differential distribution of $70-\mathrm{kD}$ heat shock protein in atherosclerosis: Its potential role in arterial SMC survival. Arterioscler Thromb Vasc Biol 1995; 15(1): 27-36. [DOI: 10.1161/01.ATV.15.1.27]; [PMID: 7749813]

296 Frostegard J, Kjellman B, Gidlund M, Andersson B, Jindal S, Kiessling R. Induction of heat shock protein in monocytic cells by oxidized low density lipoprotein. Atherosclerosis 1996; 121(1): 93-103. [DOI: 10.1016/0021-9150(95)05706-4]; [PMID: 8678928]

297 Zhu W, Roma P, Pellegatta F, Catapano AL. Oxidized-LDL induce the expression of heat shock protein 70 in human endothelial cells. Biochem Biophys Res Commun 1994; 200(1): 389-394. [DOI: 10.1006/bbrc.1994.1461]; [PMID: 8166710]

298 Zhu WM, Roma P, Pirillo A, Pellegatta F, Catapano AL. Oxidized LDL induce hsp70 expression in human smooth muscle cells. FEBS Lett 1995; 372(1): 1-5. [DOI: 10.1016/00145793(95)00834-V]; [PMID: 7556627]

$299 \mathrm{Xu}$ Q. Role of heat shock protein in atherosclerosis. Arterioscler Thromb Vasc Biol 2002; 22(10): 1547-1559. [DOI: 10.1161/01. ATV.0000029720.59649.50]; [PMID: 12377729]

300 Svensson P-A, Asea A, Englund MCO, Bausero MA, Jernas M, Wiklund $\mathrm{O}$, et al. Major role of HSP70 as a paracrine inducer of cytokine production in human oxidized LDL treated macrophages. Atherosclerosis 2006; 185(1): 32-38. [DOI: 10.1016/ j.atherosclerosis.2005.05.007]; [PMID: 15993884]

301 Zhang X, Xu Z, Zhou L, Chen Y, He M, Cheng L, et al. Plasma levels of Hsp70 and anti-Hsp70 antibody predict risk of acute coronary syndrome. Cell Stress Chaper 2010; 15(5): 675-686. [DOI: 10.1007/s12192-010-0180-3 PMID: 20300983]

302 Pockley AG, Georgiades A, Thulin T, de Faire U, Frostegard
J. Serum heat shock protein 70 levels predict the development of atherosclerosis in subjects with established hypertension. Hypertension 2003; 42(3): 235-238. [DOI: 10.1161/01. HYP.0000086522.13672.23]; [PMID: 12900429]]

303 Rockley AG, Calderwood SK, Multhoff G. The atheroprotective properties of Hsp70: a role for Hsp70-endothelial interactions? Cell Stress Chaper 2009; 14(6): 543-553. [DOI: 10.1007/s12192009-0113-1]; [PMID: 19357992]

304 Gonzalez-Ramos M, Calleros L, Lopez-Ongil S, Raoch V, Griera M, Rodriguez-Puyol M, et al. HSP70 increases extracellular matrix production by human vascular smooth muscle through TGF- $\beta 1$ up-regulation. Int J Biochem Cell Biol 2013; 45(2): 232242. [DOI: 10.1016/j.biocel.2012.10.001]; [PMID: 23084979]

305 Muzio M, Bosisio D, Polentarutti N, D’Amico G, Stoppaciaro A, Mancinelli R, et al. Differential expression and regulation of Tolllike receptors (TLR) in human leukocytes: selective expression of TLR3 in dendritic cells. J Immunol 2000; 164(11): 5998-6004. [DOI: 10.4049/jimmunol.164.11.5998]; [PMID: 10820283]

306 Visintin A, Mazzoni A, Spither JH, Wyllie DH, Dower SK, Segal DM. Regulation of Toll-like receptors in human monocytes and dendritic cells. J Immunol 2001; 166: 249-255. [DOI: 10.1049/ jimmunol.166.1.249]; [PMID: 11123299]

307 Debierre-Grockiego F, Campos MA, Azzouz N, Schmidt J, Bieker U, Resende MG, et al. Activation of TLR2 and TLR4 by glycosylphosphatidylinositols derived from Toxoplasma gondii. J Immunol 2007; 179(2): 1129-1137. [DOI: 10.4049/ jimmunol.179.2.1129]; [PMID: 17617606]

308 Black M, Boothroyd JC. Lytic cycle of Toxoplasma gondii. Microbiol Mol Biol Rev 2000; 64(3): 607-623. [DOI: 10.1128/ MMBR.64.2.607-623.2000]; [PMID: 10974128]

309 Lekutis C, Ferguson DJ, Grigg ME, Camps M, Boothroyd JC Surface antigens of Toxoplasma gondii : variations on the theme. Int J Parasitol 2001; 31(12): 1285-1292. [DOI: 10.1016/S00207519(01)00261-2]; [PMID: 11566296]

310 Wichroski MJ, Ward GE. Biosynthesis of glycophosphatidylinositol is essential to the survival of the protozoan parasite Toxoplasma gondii. Eukaryot Cell 2003; 2(5): 1132-1136. [DOI: 10.1128/EC.2.5.1132-1136.2003]; [PMID: 14555496]

311 Debierre-Grockiego F, Azzouz N, Schmidt J, Dubremetz JF, Geyer H, Geyer R, et al. Roles of glycophospatidylinositols of Toxoplasma gondii: induction of tumor necrosis factor-a production in macrophages. J Biol Chem 2003; 278(35): 3297832993. [DOI: 10.1074/jbc.M304791200]; [PMID: 12815041]

312 Yarovinsky F, Sher A. Toll-like receptor recognition of Toxoplasma gondii. Int $J$ Parasitol 2006; 36(3): 255-259. [DOI: 10.1016/j.ijpara.2005.12.003]; [PMID: 16476433]

313 Denkers EY, Butcher BA. Toxoplasma gondii parasites take control within host cells. Microbe 2012; 7(8): 360-365.

314 Lee CW, Bennouna S, Denkers EY. Screening for Toxoplasma gondii-regulated transcriptional responses in lipopolysaccharideactivated macrophages. Infect Immun 2006; 74(3): 1916-1923. [DOI: 10.1128/IAI.74.3.1916-1923.2006]; [PMID: 16495567]

315 Butcher BA, Kim L, Johnson PF, Denkers EY. Toxoplasma gondii tachyzoites inhibit proinflammatory cytokine induction in infected macrophages by preventing nuclear translocation of the

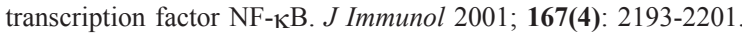
[DOI: 10.4049/jimmunol.167.4.2193]; [PMID: 11490005]

316 Bennouna S, Sukhumavasi W, Denkers EY. Toxoplasma gondi inhibits toll-like receptor 4 ligand-induced mobilization of intracellular tumor necrosis factor alpha to the surface of mouse peritoneal neutrophils. Infect Immun 2006; 74(7): 4274-4281. [DOI: 10.1128/IAI.01573-05]; [PMID: 16790802]

317 Aosai F, Rodriguez Pena MS, Mun HS, Fang H, Mitsunaga T, Norose $\mathrm{K}$, et al. Toxoplasma gondii-derived heat shock protein 70 stimulates maturation of murine bone marrow-derived dendritic cells via Toll-like receptor 4. Cell Stress Chaper 2006; 11(1): $13-$ 
22. [DOI: 10.1379/CSC-138R.1]; [PMID: 16572725]

318 Weiss LM, Ma YF, Takvorian PM, Tanowitz HB, Wittner M. Bradyzoite development in Toxoplasma gondii and the hsp70 stress response. Infect Immun 1998; 66(7): 3295-3302]; [PMID: 9632598]

319 Ma GY, Zhang JZ, Yin GR, Zhang JH, Meng XL, et al. Toxoplasma gondii proteomic analysis of antigenicity of soluble tachyzoite antigen Exp Parasitol 2009; 122(1): 41-46. [DOI: 10.1016/j.exppara.2009.01.011]; [PMID: 19545523]

320 Lyons RE, Johnson AM. Heat shock proteins of Toxoplasma gondii. Parasite Immunol 1995; 17(7): 353-359. [DOI: 10.1111/ j.1365-3024.1995.tb00902.x]; [PMID: 8552407]

321 Barenco PV, Lourenco EV, Cunha-Junior JP, Almeida KC, RoqueBarreira MC, Silva DA, et al. Toxoplasma gondii $70 \mathrm{kDa}$ heat shock protein: systemic detection is associated with the death of the parasites by the immune response and its increased expression in the brain is associated with parasite replication. PLOS ONE 2014; 9(5): e96527. [DOI: 10.1371/journal.pone.0096527]; [PMID: 24801069]

322 Silva NM, Gazzinelli RT, Silva DA, Ferro EA, Kasper LH, et al. Expression of Toxoplasma gondii-specific heat shock protein 70 during in vivo conversion of bradyzoites to tachyzoites. Infect Immun 1998; 66(8): 3959-3963]; [PMID: 9673266]

323 Mun HS, Aosai F, Norose K, Piao LX, Fang H, Akira S, et al. Toll-like receptor 4 mediates tolerance in macrophages stimulated with Toxoplasma gondii-derived heat shock protein 70. Infect Immun 2005; 73(8): 4634-4642. [DOI: 10.1128/IAI.73.8.46344642.2005]; [PMID: 16040976]

324 Garbe TR. Heat shock proteins and infection: interactions of pathogen and host. Experientia 1992; 48(7): 635-639]; [PMID: 1639171]

325 Polla BS. Heat shock proteins in host-parasite interactions. Immunol Today 1991; 12(3): A38-A41. [DOI: 10.1016/S01675699(05)80011-8]; [PMID: 8564358]

326 Young DB, Mehlert A, Smith D. Stress proteins and infectious diseases. In: R Morimoto, A Tissieres, C Georgopoulos (eds) Stress Proteins in Biology and Medicine. Cold Spring Harbor Laboratory Press, Cold Spring Harbor, NY, 1990; pp. 131-166.

327 Aosai F, Chen M, Kang H-K, Mun H-S, Norose K, Piao SX, et al. Toxoplasma gondii-derived heat shock protein HSP70 functions as a B cell mitogen. Cell Stress Chaper 2002; 7(4): 357-364]; [PMID: 12653480]

328 Mun HS, Aosai F, Norose K, Chen M, Hata H, Tagawa YI, et al. Toxoplasma gondii Hsp70 as a danger signal in Toxoplasma gondii-infected mice. Cell Stress Chaper 2000; 5(4): 328-335]; [PMID: 11048655]

329 Chen M, Aosai F, Mun HS, Norose K, Hata H, Yano A. AntiHSP70 autoantibody formation by B-1 cells in Toxoplasma gondii-infected mice. Infect Immun 2000; 68(9): 4893-4899]; [PMID: 10948102]

330 Kuppner MC, Gastpar R, Gelwer S, Nossner E, Ochmann $\mathrm{O}$, Schamer A, et al. The role of heat shock protein (hsp70) in dendritic cell maturation: hsp70 induces the maturation of immature dendritic cells but reduces DC differentiation from monocytic precursors. Eur J Immunol 2001; 31(5): 16021609. [DOI: 10.1002/1521-4141(200105)31.5<1602: : AIDIMMU1602>3.0.CO; 2-W]; [PMID: 11465118]

331 Akashi S, Shimazu R, Ogata H, Nagai Y, Takeda K, Kimoto M, et al. Cutting edge : cells surface expression and lipopolysaccharide signaling via the toll-like receptor 4-MD-2 complex on mouse peritoneal macrophages. J Immunol 2000; 164(7): 3471-3475. [DOI: 10.4049/jimmunol.164.7.3471]; [PMID: 10725698]

332 Sato S, Nomura F, Kawai T, Takeuchi O, Muhlradt PF,Takeda K, et al. Synergy and cross-tolerance between toll-like receptor (TRL) 2- and TRL4-mediated signaling pathways. J Immunol 2000; 165(12): 7096-7101. [DOI: 10.4049/jimmunol.165.12.7096]; [PMID: 11120839]
333 Glass CK, Witztum JL. Atherosclerosis. The road ahead. Cell 2001; 104(4): 503-516. [DOI: 10.1016/S0092-8674(01)00238-0]; [PMID: 11239408]

334 Falk E. Pathogenesis of atherosclerosis. J Am Coll Cardiol 2006; 47(8 Suppl): C7-C12. [DOI: 10.1016/jacc.2005.09.068]; [PMID: 16631513]

335 Chen CC, Chiu KT, Sun YT, Chen WC. Role of the cyclic AMP-protein kinase a pathway in lipopolysaccharide-induced nitric oxide synthase expression in RAW 264.7 macrophages. Involvement of cyclooxygenase-2. J Biol Chem 1999; 274(44): 31559-31564. [DOI: 10.1074/jbc.274.44.31559]; [PMID: 10531359]

336 Laskin DL, Pendino KJ. Macrophages and inflammatory mediators in tissue injury. Annu Rev Pharmacol Toxicol 1995; 35: 655-677. [DOI: 10.1146/annurev.pa.35.040195.003255]; [PMID: 7598511]

337 MacMicking J, Xie QW, Nathan C. Nitric oxide and macrophage function. Annu Rev Immunol 1997; 15: 323-350. [DOI: 10.1146/ annurev.immunol.15.1.323]; [PMID: 914369]

$338 \mathrm{Xu}$ Q, Hu Y, Kleindienst R, Wick G. Nitric oxide induces heatshock protein 70 expression in vascular smooth muscle cells via activation of heat shock factor 1. J Clin Invest 1997; 100(5): 10891097. [DOI: 10.1172/JCI119619]; [PMID: 9276725]

339 Kim YM, de Vera ME, Watkins SC, Billiar TR. Nitric oxide protects cultured rat hepatocytes from tumor necrosis factoralpha-induced apoptosis by inducing heat shock protein 70 expression. J Biol Chem 1997; 272(2): 1402-1411. [DOI: 10.1074/ jbc.272.2.1402]; [PMID: 8995451]

340 Malyshev IYu, Malugin AV, Golubeva LYu, Zenina TA, Manukhina EB, Mikoyan VD, et al. Nitric oxide donor induces HSP70 accumulation in the heart and in cultured cells. FEBS Lett 1996; 391(1-2): 21-23. [DOI: 10.1016/0014-5793(96)00691-6]; [PMID: 8706919]

341 Yano A, Mun HS, Chen M, Norose K, Hata H, Kobayashi M, et al. Roles of IFN- $\gamma$ on stage conversion of an obligate intracellular protozoan parasite, Toxoplasma gondii. Int Rev Immunol 2002; 21(4-5): 405-421]; [PMID: 12486821]

342 Ramji DP, Davies TS. Cytokines and atherosclerosis: key players in all stages of disease and promising therapeutic targets. Cytokine Growth Factor Rev 2015; 26(6): 673-685. [DOI: 10.1016/ j.cytogfr.2015.04.003]; [PMID: 26005197]

343 Getz GS. Thematic review series: The immune system and atherogenesis. Immune function and atherogenesis. $J$ Lipid Res 2005; 46(1): 1-10. [DOI: 10.1194/j1r.R400013-JLR200]; [PMID: 15547292]

344 Raines EW, Ferri N. The immune system and atherogenesis. Cytokines affecting endothelial and smooth muscle cells in vascular disease. J Lipid Res 2005; 46(6): 1081-1092. [DOI: 10.1194/jlr.R500004-JLR200]; [PMID: 15834121]

345 Daugherty A, Webb NR, Rateri DL, King VL. Thematic review series: The immune system and atherogenesis. Cytokine regulation of macrophage function in atherogenesis. J Lipid Res 2005; 46(9): 1812-1822. [DOI: 10.1194/j1r.R5000009-JLR200]; [PMID: $15995168]$

346 Baud V, Karin M. Signal transduction by tumor necrosis factor and its relatives. Trends Cell Biol 2001; 11(9): 372-377. [DOI: 10.1016/S0962-8924(01)02064-5]; [PMID: 11514191]

347 Branen L, Hovgaard L, Nitulescu M, Bengtsson E, Nilsson $\mathrm{J}$, Joving $\mathrm{S}$. Inhibition of tumor necrosis factor-alpha reduces atherosclerosiis in apolipoprotein E knockout mice. Arterioscler Thromb Vasc Biol 2004; 24(11): 2137-2142. [DOI: 10.1161/01. ATV.0000143933.20616.1b]; [PMID: 15345516]

348 Skoog T, Dichtl W, Boquist S, Skoglund-Andersson C, Karpe $\mathrm{F}$, Tang R, et al. Plasma tumour necrosis factor-alpha and early carotid atherosclerosis in healthy middle-aged men. Eur Heart $J$ 2002; 23(5): 376-383. [DOI: 10.1053/euhj.2001.2805]; [PMID: $11846495]$ 
349 Libby P, Hansson GK. Involvement of the immune system in human atherogenesis: current knowledge and unanswered questions. Lab Invest 1991; 64(1): 5-15]; [PMID: 1990208]

350 Furukawa K, Hori M, Ouchi N, Kihara S, Funahashi T, Matsuzawa Y, et al. Adiponectin downregulates acyl coenzym A: cholesterol acyltransferase-1 in cultured human monocyte-derived macrophages. Biochem Biophys Res Commun 2004; 317(3): 831836. [DOI: 10.1016/j.bbrc.2004.03.123]; [PMID: 15081415]

351 Tsubakio-Yamamoto K, Matsuura F, Koseki M, Oku H, Sandoval $\mathrm{JC}$, Inagaki $\mathrm{M}$, et al. Adiponectin prevents atherosclerosis by increasing cholesterol efflux from macrophages. Biochem Biophys Res Commun 2008; 375(3): 390-394. [DOI: 10.1016/ j.bbrc.2008.08.009]; [PMID: 18703020]

352 Persson J, Nilsson J, Lindholm MW. Cytokine response to lipoprotein lipid loading in human monocyte-derived macrophages. Lipids Health Dis 2006; 5: 17. [DOI: 10.1186/1476511X-5-17]; [PMID: 16800873]

353 Stollenwerk MM, Lindholm MW, Pörn-Ares MI, Larsson A, Nilsson J, Ares MP. Very low-density lipoprotein induces interleukin-1 beta expression in macrophages. Biochem Biophys Res Commun 2005; 335(2): 603-608. [DOI: 10.1016/ j.bbrc.2005.07.123]; [PMID: 16087165]

354 McLaren JE, Michael DR, Salter RC, Ashlin TG, Calder CJ, Miller AM, et al. IL-33 reduces macrophage foam cell formation. J Immunol 2010; 185(2) : 1222-1229. [DOI: 10.4049/ jimmunol.1000520]; [PMID: 20543107]

355 Schmitz J, Owyang A, Oldham E, Song Y, Murphy E, McClanahan TK, et al. IL-33, an interleukin-1-like cytokine that signals via IL-1 receptor-related protein ST2 and induces T helper type 2-associated cytokines. Immunity 2005; 23(5): 479-490. [DOI: 10.1016/j.immuni.2005.09.015]; [PMID: 16286016]

356 Kakkar R, Lee RT. The IL-33/ST2 pathway: therapeutic target and novel biomarker. Nat Rev Drug Discov 2008; 7(10): 827-840. [DOI: 10.1038/nrd2660]; [PMID: 18827826]

357 Haraldsen G, Balogh J, Pollheimer J, Sponheim J, Küchler AM. Interleukin-33 - cytokine of dual function or novel alarmin? Trends Immunol 2009; 30(5): 227-233. [DOI: 10.1016/ j.it.2009.03.003]; [PMID: 19359217]

358 Manetti M, Ibba-Manneschi L, Liakouli V, Guiducci S, Milia AF, Benelli G, et al. The IL-1-like cytokine IL33 and its receptor ST2 are abnormally expressed in the affected skin and visceral organs of patients with systemic sclerosis. Ann Rheum Dis 2010; 69(3): 598-605. [DOI: 10.1136/ard.2009.119321]; [PMID: 19778913]

359 Feingold KR, Staprans I, Memon R, Moser AH, Shigenaga $\mathrm{JK}$, Doerrler W, et al. Endotoxin rapidly induces changes in lipid metabolism that produce hypertriglyceridemia: low doses stimulate hepatic triglyceride production while high doses inhibit clearance. J Lipid Res 1992; 33(12): 1765-1776.]PMID: 1479286]

360 Khovidhunkit W, Kim MS, Memon RA, Shigenaga JK, Moser $\mathrm{AH}$, Feingold KR, et al. Effects of infection and inflammation on lipid and lipoprotein metabolism: mechanisms and consequences to the host. J Lipid Res 2004; 45(7): 1169-1196. [DOI: 10.1194/j1r. R300019-JLR200]; [PMID: 15102878]

361 Feingold KR, Soued M, Serio MK, Moser AH, Dinarello CA, Grunfeld C. Multiple cytokines stimulate hepatic lipid synthesis in vivo. Endocrinology 1989; 125(1): 267-274. [DOI: 10.1210/endo125-1-267]; [PMID: 2661207]

362 Evans RD, Ilic V, Williamson DH. Effects of platelet activating factor on lipid metabolism in rats in vivo. Origin of the hypertrigliceridaemia. Biochem J 1991; 280(Pt 2): 541-543. [DOI: 10.1042/bj2800541]; [PMID: 1747129]

363 Grunfeld C, Verdier JA, Neese R, Moser AH, Feingold KR. Mechanism by which tumor necrosis factor stimulates hepatic fatty acid synthesis in vivo. J Lipid Res (1988) 29(10): 1327-35]; [PMID: 2906959]

364 Grunfeld C, Soued M, Adi S, Moser AH, Fiers W, Dinarello CA, et al. Interleukin 4 inhibits stimulation of hepatic lipogenesis by tumor necrosis factor, interleukin 1 , and interleukin 6 but not by interferon-alpha. Cancer Res 1991; 51(11): 2803-2807. [PMID: 2032220]

365 Feingold KR, Hardardottir I, Memon R, Krul EJ, Moser AH, Taylor JM, et al. Effect of endotoxin on cholesterol biosynthesis and distribution in serum lipoproteins in Syrian hamsters. J Lipid Res 1993; 34(12): 2147-2158. [PMID: 8301233]

366 Feingold KR, Pollock AS, Moser AH, Shigenaga JK, Grunfeld C. Discordant regulation of proteins of cholesterol metabolism during the acute phase response. J Lipid Res 1995; 36(7): $1474-$ 1482]; [PMID: 7595071]

$367 \mathrm{Xu} \mathrm{N}$, Nilsson A. Endotoxin inhibits catabolism of low density lipoproteins in vivo: an experimental study in the rat. Scand J Clin Lab Invest 1996; 56(1): 53-61]; [PMID: 8850173]

368 Araujo FG, Slifer T. Different strains of Toxoplasma gondii induce different cytokine responses in CBA/Ca mice. Infect Immun 2003; 71(7): 4171-4174. [DOI: 10.1128/IAI.71.7.4171-4174.2003]; [PMID: 12919111]

369 Suzuki Y, Conley FK, Remington JS. Differences in virulence and development of encephalitis during chronic infection vary with the strain of Toxoplasma gondii. J Infect Dis 1989; 159(4): 790794]; [PMID: 2926171]

370 Palucka K, Banchereau J. Dendritic cells: a link between innate and adaptive immunity. J Clin Immunol 1999; 19(1): 12-25]; [PMID: 10080101]

371 Reis e Souza C, Sher A, Kaye P. The role of dendritic cells in the induction and regulation of immunity to microbial infection. Curr Opin Immunol 1999; 11(4): 392-399. [DOI: 10.1016/S09527915(99)80066-1]; [PMID: 10448137]

372 Bliss SK, Marshall AJ, Zhang Y, Denkers EY. Human polymorphonuclear leukocytes produce IL-12, TNF- $\alpha$, and the chemokines macrophage-inflammatory protein- $1 \mathrm{a}$ and $-1 \mathrm{~b}$ in response to Toxoplasma gondii antigens. J Immunol 1999; 162(12): 7369-7375. [PMID: 10358188]

373 Filisetti D, Candolfi E. Immune response to Toxoplasma gondii. Ann Ist Super Sanita 2004; 40(1): 71-80]; [PMID: 15269455]

374 Grau G, Tacchini-Cooler F, Piguet P-F. Is TNF beneficial or deleterious in Toxoplasmic encephalitis? Parasitol Today 1992; 8(10): 322-324. [DOI: 10.1016/0169-4758(92)90062-7]; [PMID: 15463526]

375 Locksley RM. Interleukin 12 in host defense against microbial pathogens. Proc Natl Acad Sci USA 1993; 90(13): 5879-5880]; [PMID: 8100997]

376 Sibley LD, Adams LB, Fukutomi AY, Krahenbuhl JL. Tumor necrosis factor-a triggers antitoxoplasmal activity in IFN- $\gamma$ primed macrophages. J Immunol 1991; 147(7): 2340-2345. [PMID: 1918966]

377 Johnson LL. A protective role for endogenous tumor necrosis factor in Toxoplasma gondii infection. Infect Immun 1992; 60(5): 1979-1983]; [PMID: 1563790]

378 Gazzinelli RT, Wysocka M, Hieny S, Scharton-Kersten T, Cheever A, Kühn R, et al. In the absence of endogenous IL-10, mice acutely infected with Toxoplasma gondii succumb to a lethal immune response dependent upon CD4+ T cells and accompanied by overproduction of IL-12, IFN- $\gamma$, and TNF- $\alpha$. J Immunol 1996; 157(2): 798-805]; [PMID: 8752931]

379 Chang HR, Grau GE, Pechere JC. Role of TNF and IL1 in infections with Toxoplasma gondii. Immunology 1990; 69(1): 3337. [PMID: 2107144]

380 Lüder CGK, Algner M, Lang C, Bleicher N, Gross U. Reduced expression of the inducible nitric oxide synthase after infection with Toxoplasma gondii facilitates parasite replication in activated murine macrophages. Int J Parasitol 2003; 33(8): 833-844. [DOI: 10.1016/S0020-7519(03)00092-4]; [PMID: 12865]

381 Mordue DG, Hakansson S, Niesman I, Sibley LD. Toxoplasma gondii resides in a vacuole that avoids fusion with host cell endocytic and exocytic vesicular trafficking pathways. Exp 
Parasitol 1999; 92(2): 87-99. [DOI: 10.1006/expr.1999.4412]; [PMID: 10366534]

382 Sibley LD, Lawson R, Krahenbuhl JL. Phagosome acidification blocked by intracellular Toxoplasma gondii. Nature 1990; 315(6018): 416-419. [PMID: 2860567]

383 Silver JS, Stumhofer JS, Passos S, Ernst M, Hunter CA. IL-6 mediates the susceptibility of glycoprotein 130 hypermorphs to Toxoplasma gondii. J Immunol 2011; 187(1): 350-360. [DOI: 10.4049/jimmunol.1004144]; [PMID: 21606248]

384 Casella G, Garzetti L, Gatta A, Finardi A, Maiorino C, Ruffini $\mathrm{F}$, et al. IL4 induces IL6-producing M2 macrophages associated to inhibition of neuroinflammation in vitro and in vivo. $J$ Neuroinflam 2016; 13(1): 139. [DOI: 10.1186/s12974-016-05965]; [PMID: 27266518]

385 Gabay C. Interleukin-6 and chronic inflammation. Arthritis Res Ther 2006; 8(Suppl 2): S3. [DOI: 10.1186/ar1917]; [PMID: 16899107]

386 Gauldie J, Richards C, Harnish D, Lansdorp P, Baumann H. Interferon b2/B-cell stimulatory factor type 2 shares identity with monocyte-derived hepatocyte-stimulating factor and regulates the major acute phase protein response in liver cells. Proc Natl Acad Sci USA 1987; 84(20): 7251-7255]; [PMID: 2444978]

387 Kaplanski G, Marin V, Montero-Julian F, Mantovani A, Farnarier C. IL-6: a regulator of the transition from neutrophil to monocyte recruitment during inflammation. Trends Immunol 2003; 24(1): 25-29. [DOI: 10.1016/S1471-4906(02)00013-3]; [PMID: 12495721]

388 Graeve L, Baumann M, Heinrich PC. Interleukin-6 in autoimmune disease: role of IL-6 in physiology and pathology of the immune defense. Clin Invest 1993; 71(8): 664-671]; [PMID: 8210666]

389 Grimble RF. Nutritional modulation of cytokine biology. Nutrition 1998; 14(7-8): 634-640. [DOI: 10.1016/S0899-9007(98)00010-0]; [PMID: 9684268]

390 Tang CH, Lu DY, Yang RS, Tsai HY, Kao MC, Fu WM, et al. Leptin-induced IL-6 production is mediated by leptin receptor, insulin receptor substrate-1, phosphatidylinositol 3-kinase, Akt, $\mathrm{NF}-\kappa \mathrm{B}$, and p300 pathway in microglia. J Immunol 2007; 179(2): 1292-1302. [DOI: 10.4049/jimmunol.179.2.1292]; [PMID: $17617622]$

391 Frisdal E, Lesnik P, Olivier M, Robillard P, Chapman MJ, Huby $\mathrm{T}$, et al. Interleukin-6 protects human macrophages from cellular cholesterol accumulation and attenuates the proinflammatory response. J Biol Chem 2011; 286(35): 30926-30936. [DOI: 10.1074/jbc.M111.264325]; [PMID: 21757719]

392 Beaman MH, Hunter CA, Remington JS. Enhancement of intracellular replication of Toxoplasma gondii by IL-6.

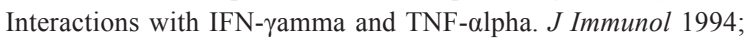
153(10): 4583-4587. [PMID: 7963530]

393 Fang Y, Tan Y, Zhang Y, Li J. Effect of IL-6 on the multiplication of Toxoplasma gondii. Zhongguo Ji Sheng Chong XueYu Ji Sheng Chong Bing Za Zhi 1999; 17(2): 106-108]; [PMID: 12563793]

394 Kolodgie FD, Burke AP, Skorija KS, Ladich E, Kutys R, Makuria AT, et al. Lipoprotein-associated phospholipase A2 protein expression in the natural progression of human coronary atherosclerosis. Arterioscler Thromb Vasc Biol 2006; 26(11): 2523-2529. [DOI: 10.1161/01.ATV.0000244681.72738.bc]; [PMID: 16960105]

395 Saffer LD, Long Krug SA, Schwartzman JD. The role of phospholipase in host cell penetration by Toxoplasma gondii. Am J Trop Med Hyg 1989; 40(2): 145-149. [PMID: 2919724]

396 Buzas EI, György B, Nagy G, Falus A, Gay S. Emerging role of extracellular vesicles in inflammatory disorders. Nat Rev Rheumatol 2014; 10(6): 356-364. [DOI: 10.1038/ nrrheum.2014.19]; [PMID: 24535546]

397 Bhatnagar S, Shinagawa K, Castellino FJ, Schorey JS. Exosomes released from macrophages infected with intracellular pathogens stimulate a proinflammatory response in vitro and in vivo. Blood
2007; 110(9): 3234-3244. [DOI: 10.1182/blood-2007-03-079152]; [PMID: 17666571]

398 Van Niel G, Porto-Carreiro I, Simoes S, Raposo G. Exosomes: a common pathway for a specialized function. $J$ Biochem (Tokyo) 2006; 140(1): 13-21. [DOI: 10.1093/jb/mvj128]; [PMID: 16877764]

399 Raposo G, Nijman HW, Stoorvogal W, Ljelendekker R, Harding $\mathrm{CV}$, Melief CJ, et al. B lymphocytes secrete antigen presenting vesicles. J Exp Med 1996; 183(3): 1161-1172. [DOI: 10.1084/ jem.183.3.1161]; [PMID: 8642258]

400 Mignot G, Roux S, Thery C, Segura E, Zitvogel L. Prospects for exosomes in immunotherapy of cancer. J Cell Mol Med 2006; 10(2): 376-388. [DOI: 10.1111/j.1582-4934.2006.tb00406]; [PMID: 16796806]

401 Chaput N, Flament C, Viaud S, Taieb J, Roux S, Spatz A, et al. Dendritic cell-derived exosomes: biology and clinical implementations. J Leukoc Biol 2006; 80(3): 471-478. [DOI: 10.1189/jlb.0206094]; [PMID: 16809645]

402 Aline F, Bout D, Amigorena S, Roingeard P, Dimier-Poisson I. Toxoplasma gondii antigen-pulsed-dendritic cell-derived exosomes induce a protective immune response against $T$. gondii infection. Infect Immun 2004; 72(7): 4127-4137]; [PMID: 15213158 [DOI: 10.1128/IAI.727.4127-4137.2004]

403 Pope SM, Lässer C. Toxoplasma gondii infection of fibroblasts causes the production of exosome-like vesicles containing a unique array of mRNA and miRNA transcripts compared to serum starvation. J Extracell Vesicles 2013; 2: 22484. [DOI: 10.3402/jev. v210.22484]; [PMID: 24363837]

404 Blanch A, Robinson F, Watson IR, Cheng LS, Irwin MS. Eukaryotic translation elongation factor 1-alpha inhibits p53 and p73 dependent apoptosis and chemotherapy sensitivity. PLoS ONE 2013; 8(6): e66436. [DOI: 10.1371/journal.pone.0066436]; [PMID: 23799114]

405 Tsai NP, Wilkerson JR, Guo W, Maksimova MA, DeMartino $\mathrm{GN}$, Cowan CW, et al. Multiple autism-linked genes mediate synapse elimination via proteosomal degradation of a synaptic scaffold PSD-95. Cell 2012; 151(7): 1581-1594. [DOI: 10.1016/ j.cell.2012.11.040]; [PMID: 23260144]

406 Sakane A, Honda K, Sasaki T. Rab13 regulates neurite outgrowth in PC12 cells through its effector protein, JRAB/MICAL-L2. Mol Cell Biol 2010; 30(4): 1077-1087. [DOI: 10.1128/MCB.0106709]; [PMID: 20008558]

407 Jüngel A, Distler O, Schulze-Horsel U, Huber LC, Ha HR, Simmen B, al. Microparticles stimulate synthesis of prostaglandin E(2) via induction of cyclooxygenase 2 and microsomal prostaglandin E synthase 1. Arthritis Rheum 56(11): 3564-3574. [DOI: 10.1002/art.22980]; [PMID: 17968936]

408 Chistiakov DA, Orekhov AN, Bobryshev YV. Extracellular vesicles and atherosclerotic disease. Cell Mol Life Sci 2015; 72(14): 2697-2708. [DOI: 10.1007/s00018-015-1906-2]; [PMID: 25894694]

409 Szotowski B, Antoniak S, Goldin-Lang P, Tran QV, Pels K, Rosenthal $\mathrm{P}$, et al. Antioxidative treatment inhibits the release of thrombogenic tissue factor from irradiation- and cytokine-induced endothelial cells. Cardiovasc Res 2007; 73(4): 806-812. [DOI: 10.1016/j.cardiores.2006.12.018]; [PMID: 17266944]

410 Martel C, Li W, Fulp B, Platt AM, Gautier EL, Westerterp M, et al. Lymphatic vasculature mediates macrophage reverse cholesterol transport in mice. J Clin Invest 2013; 123(4): 15711579. [DOI: 10.1172/JCI63685]; [PMID: 23524964]

411 Eliska O, Eliskova M, Miller AJ. The absence of lymphatics in normal and atherosclerotic coronary arteries in man: a morphologic study. Lymphology 2006; 39(2): 76-83]; [PMID: 16910098]

412 Torrisi JS, Hespe GE, Cuzzone DA, Savetsky IL, Nitti MD, Gardenier JC, et al. Inhibition of inflammation and iNOS improves lymphatic function in obesity. Sci Rep 2016; 6: 19817. 
[DOI: 10.1038/srep19817]; [PMID: 26796537]

413 Milasan A, Tessandier N, Tan S, Brisson A, Boilard E, Martel C. Extracellular vesicles are present in mouse lymph and their level differs in atherosclerosis. J Extracell Vesicles 2016; 5: 31427. [DOI: 10.3402/jev.v5.31427]; [PMID: 27664155]

414 Tanabe K, Asai T, Kimata I, Takada S. Penetration of maturating red blood cells by Toxoplasma gondii. Microbiology 1979; 113(2): 433-437. [DOI: 10.1099/00221287-113-2-433]; [PMID: 512638]

415 Djaldetti M, Bessler H. On the phagocyting properties of human platelets. Med Con 2011; 6(3): 9-12. ISSN online 2068-8369

416 DaMatta RA, Seabra SH, de Souza W. Further studies on the phagocytic capacity of chicken thrombocytes. J Submicrosc Cytol Pathol 1998; 30(2): 271-277.]; [PMID: 9648290

417 Yong EC, Chi EY, Fritsche TR, Henderson WR Jr. Human platelet-mediated cytotoxicity against Toxoplasma gondii: role of thromboxane. J Exp Med 1991; 173(1): 65-78. [DOI: 10.1084/ jem.173.1.65]; [PMID: 1808664]

418 Malkwitz I, Berndt A, Zhang R, Daugschies A, Bangoura B. Replication of Toxoplasma gondii in chicken erythrocytes and thrombocytes compared to macrophages. Parasitol Res 2016; Oct 3. [DOI: 10.1007/300436-016-05268-y]; [PMID: 27696227]

419 McCabe RE, Remington JS. Toxoplasma gondii. $3^{\text {rd }}$ ed. In: Principles and Practice of Infectious Diseases. GL Mandell, RG Douglas, JE Bennett (eds). Churchill Livingstone, Inc., New York, 1990; pp. 2090-2103.

420 Suzuki Y, Orellana MA, Schreiber RD, Remington JS. Interferongamma. The major mediator of resistance against Toxoplasma gondii. Science 1988; 240(4851): 516-8. [DOI: 10.1126/ science.3128869]; [PMID: 3128869]

421 Libregts SF, Gutierrez L, de Bruin AM, Wensveen FM, Papadopoulos $\mathrm{P}$, van Ijcken $\mathrm{W}$, et al. Chronic IFN- $\gamma$ production in mice induces anemia by reducing erythrocyte life span and inhibiting erythropoiesis through an IRF-1/PU. axis. Blood 2011; 118(9): 2578-2588. [DOI: 10.1182/blood-2010-10-315218]; [PMID: 21725055]

422 Mullarky IK, Szaba FM, Kummer LW, Wilhelm LB, Parent MA, Johnson LL, et al. Gamma interferon suppresses erythropoiesis via interleukin-15. Infect Immun 2007; 75(5): 2630-2633. [DOI: 10.1128/IAI.01836-06]; [PMID: 17307940]

423 Wang Z, Zhang D-X, Zhao Q. Infection-stimulated anemia results primarily from interferon gamma-dependent, signal transducer and activator of transcription 1-independent red cell loss. Chinese Med J (Engl) 2015; 128(7): 948-955. [DOI: 10.4103/03666999.154303]; [PMID: 25836617]

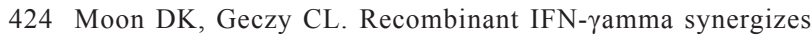
with lipopolysaccharide to induce macrophage membrane procoagulants. J Immunol 1988; 141(5): 1536-1542. [PMID: 2457619]

425 Schwager I, Jungi TW. Effect of human recombinant cytokines on the induction of macrophage procoagulant activity. Blood 1994; 83(1): 152-160]; [PMID: 8274733]

426 Hamilton JA, Whitty GA, Last K, Royston AK, Hart PH, Burgess DR. Interleukin-4 suppresses plasminogen activator inhibitor-2 formation in stimulated human monocytes. Blood 1992; 80(1): 121-125]; [PMID: 1611080]

427 Del Prete G, De Carli M, Lammel RM, D'Elios M, Daniel KC, Giusti B, et al. Th1 and Th2 T-helper cells exert opposite regulatory effects on procoagulant activity and tissue factor production by human monocytes. Blood 1995; 86(1): 250-257]; [PMID: 7795230]

428 Ahrenholz DH, Simmons RL. Fibrin in peritonitis. I. Beneficial and adverse effects of fibrin in experimental E. coli peritonitis. Surgery 1980; 88(1): 41-47]; [PMID: 6992321]

429 Johnson LL, Berggren KN, Szaba FM, Chen W, Smiley ST. Fibrin-mediated protection against infection-stimulated immunopathology. J Exp Med 2003; 19(6)7: 801-806. [DOI: 10.1084/jem.20021493]; [PMID: 12629066]
430 Mullarky IK, Sztaba FM, Berggren KN, Parent MA, Kummer LW, Chen W, et al. Infection-stimulated fibrin deposition controls hemorrhage and limits hepatic bacterial growth during listeriosis. Infect Immun 2005; 73(7): 3888-3895. [DOI: 10.1128/ IAI.73.7.3888-3895.2005]; [PMID: 15972474]

431 Dunn DL, Simmons RL. Fibrin in peritonitis. III. The mechanism of bacterial trapping by polymerizing fibrin. Surgery 1982; 92(3): 513-519]; [PMID: 7051387]

432 Echtenacher B, Weigl K, Lehn N, Männel DN. Tumor necrosis factor-dependent adhesions as a major protective mechanism early in septic peritonitis in mice. Infect Immun 2001; 69(6): 3550-3555. [DOI: 10.1128/IAI.69.6.3550-3555.2001]; [PMID: 11349012]

433 Rotstein OD. Role of fibrin deposition in the pathogenesis of intraabdominal fection. Eur J Clin Microbiol Infect Dis 1992 ; 11(11): 1064-1068. [PMID : 1295760]

434 Mullarky IK, Szaba FM, Berggren KN, Kummer LW, Wilhelm LB, Parent MA, et al. Tumor necrosis factor alpha and gamma interferon, but not hemorrhage or pathogen burden, dictate levels of protective fibrin deposition during infection. Infect Immun 2006; 74(2): 1181-1188. [DOI: 10.1128/IAI.74.2.11811188.2006]; [PMID: 16428767]

435 Brett J, Gerlach H, Nawroth P, Steinberg S, Godman G, Stern D. Tumor necrosis factor/cachectin increases permeability of endothelial cell monolayers by a mechanism involving regulatory G proteins. J Exp Med 1989; 169(6): 1977-1991. [DOI: 10.1084/ jem.169.6.1977]; [PMID: 2499653]

436 Clauss M, Sunderkötter C, Sveinbjörnsson B, Hippenstiel S, Willoweit A, Marino M, et al. A permissive role for tumor necrosis factor in vascular endothelial growth factor-induced vascular permeability. Blood 2001; 97(5): 1321-1329. [DOI: 10.1182/ blood.V97.5.1321]; [PMID: 11222376]

437 Friedl J, Puhlmann M, Bartlett DL, Libutti SK, Turner EN, Gnant MF, et al. Induction of permeability across endothelial cell monolayers by tumor necrosis factor (TFN) occurs via a tissue factor-dependent mechanism: relationship between the procoagulant and permeability effects of TNF. Blood 2002; 100(4): 1334-1339]; [PMID: 12149215]

438 Nawroth PP, Stern DM. Modulation of endothelial cell hemostatic properties by tumor necrosis factor. J Exp Med 1986; 163(3): 740745. [DOI: 10.1084/jem.163.3.740]; [PMID: 3753996]

439 Scarpati EM, Sadler JE. Regulation of endothelial cell coagulan properties. Modulation of tissue factor, plasminogen activator inhibitors, and thrombomodulin by phorbol 12-myristate 13-acetate and tumor necrosis factor. J Biol Chem 1989; 264(34): 20705-20713. [PMID: 2555368]

440 Dosne AM, Dubor F, Lutcher F, Parant M, Chedid L. Tumor necrosis factor (TNF) stimulates plasminogen activator inhibitor (PAI) production by endothelial cells and decreases blood fibrinolytic activity in the rat. Thromb Res Suppl 1988; 8: 115-22. [PMID: 3144762]

441 Medcalf RL, Kruithof EK, Schleuning WD. Plasminogen activator inhibitor 1 and 2 are tumor necrosis factor/cachectin-responsive genes. J Exp Med 1988; 168(2): 751-759. [DOI: 10.1084/ jem.168.2.751]; [PMID: 3137305]

442 van Hinsbergh VW, Kooistra T, van den Berg EA, Princen HM, Fiers W, Emeis JJ. Tumor necrosis factor increases the production of plasminogen activator inhibitor in human endothelial cells in vitro and in rats in vivo. Blood 1988; 72(5): 1467-73]; [PMID: 3140909]

443 van Hinsbergh VW, Kooistra T, Scheffer MA, Hajo van Bockel J, van Muijen GN. Characterization and fibrynolytic properties of human omental tissue mesothelial cells. Comparison with endothelial cells. Blood 1990; 75(7): 1490-7]; [PMID: 2197884]

444 Kitching AR, Holdsworth SR, Ploplis VA, Plow EF, Collen D, Carmeliet $\mathrm{P}$, et al. Plasminogen and plasminogen activators protect against renal injury in crescentic glomerulonephritis. $J$ Exp Med 1997; 185(5): 963-968. [DOI: 10.1084/jem.185.5.963]; [PMID: 
9120402]

445 Busso N, Peclat V, Van Ness K, Kolodziesczyk E, Degen J, Bugge $\mathrm{T}$, et al. Exacerbation of antigen-induced arthritis in urokinasedeficient mice. J Clin Invest 1998; 102(1): 41-50. [DOI: 10.1172/ JCI2312]; [PMID: 9649555]

446 Yang YH, Carmeliet P, Hamilton JA. Tissue-type plasminogen activator deficiency exacerbates arthritis. J Immunol 2001; 167(2): 1047-1052.[DOI: 10.4049/jimmunol.167.2.1047]; [PMID: $11441114]$

447 Lou XJ, Boonmark NW, Horrigan FT, Degen JL, Lawn RM. Fibrinogen deficiency reduces vascular accumulation of apolipoprotein(a) and development of atherosclerosis in apolipoprotein(a) transgenic mice. Proc Natl Acad Sci USA 1998; 95(21): 12591-12595. [DOI: 10.1073/pnas.95.21.12591]; [PMID: 9770530]

448 Szaba FM, Smiley ST. Roles for thrombin and fibrin(ogen) in cytokine/chemokine production and macrophage adhesion in vivo. Blood 2002; 99(3): 1053-1059. [DOI: 10.1182/blood.V99.3.1053]; [PMID: 11807012]

449 Carmeliet P, Stassen JM, Schoonjans L, Ream B, van den Oord JJ, De Mol M, et al. Plasminogen activator inhibitor-1 gene-deficient mice. II. Effects on hemostasis, thrombosis, and thrombolysis. $J$ Clin Invest 1993; 92(6): 2756-2760. [DOI: 10.1172/JCI116893]; [PMID: 8254029]

450 Nilsson L, Banfi C, Diczfalusy U, Tremoli E, Hamsten A, Eriksson P. Unsaturated fatty acids increase plasminogen activator inhibitor-1 expression in endothelial cells. Arterioscler Thromb Vasc Biol 1998; 18(11): 1679-1685. [DOI: 10.1161/01. ATV.18.11.1679]; [PMID: 9812904]

451 Hamsten A, Eriksson P. Fibrinolysis and atherosclerosis. Baillieres Clin Haematol 1995; 8(2): 345-363]; [PMID: 7549067]

452 Stiko-Rahm A, Wiman B, Hamsten A, Nilsson J. Secretion of plasminogen activator inhibitor-1 from cultured human umbilical vein endothelial cells is induced by very low density lipoprotein. Arteriosclerosis 1990; 10(6): 1067-1073. [DOI: org/10.1161/01. ATV.10.6.1067]; [PMID: 2244856]

453 Mussoni L, Mannucci L, Sirtori M, Camera M, Maderna P, Sironi $\mathrm{L}$, et al. Hypertriglyceridemia and regulation of fibrinolytic activity. Arterioscler Thromb 1992; 12(1): 19-27. [DOI: 01.ATV.12.1.19]; [PMID: 1310024]

454 Kaneko T, Wada H, Wakita Y, Minamikawa K, Nakase T, Mori Y, et al. Enhanced tissue factor activity and plasminogen activator inhibitor-1 antigen in human vein endothelial cells incubated with lipoproteins. Blood Coagul Fibrinolysis 1994; 5(3): 385-392]; [PMID: 8075310]

455 Sironi L, Mussoni L, Prati L, Baldassarre D, Camera M, Banfi C, et al. Plasminogen activator inhibitor type-1 synthesis and mRNA expression in HepG2 cells are regulated by VLDL. Arterioscler Thromb Vasc Biol 1996; 16(1): 89-96. [DOI: org/10.1161/01. ATV.16.1.89]; [PMID: 8548432]

456 Hamsten A, Wiman B, de Faire U, Blombäck M. Increased plasma levels of a rapid inhibitor of tissue plasminogen activator in young survivors of myocardial infarction. $N$ Engl J Med 1985; 313(25): 1557-1563. [DOI: 10.1056/NEJM198512193132501]; [PMID: 3934538]

457 Mehta J, Mehta P, Lawson D, Saldeen T. Plasma tissue plasminogen activator inhibitor levels in coronary artery disease: correlation with age and serum triglyceride concentrations. $J \mathrm{Am}$ Coll Cardiol 1987; 9(2): 263-268]; [PMID: 3100598]

458 Asplund-Carlson A, Hamsten A, Wiman B, Carlson LA. Relationship between plasma plasminogen activator inhibitor-1 activity and VLDL triglyceride concentration, insulin levels nd insulin sensitivity: studies in randomly selected normo- and hypertriglyceridaemic men. Diabetologia 1993; 36(9): 817-825]; [PMID: 8104834]

459 Eriksson P, Nilsson L, Karpe F, Hamsten A. Very-low-density lipoprotein response element in the promoter region of the human plasminogen activator inhibitor-1 gene implicatedin the impaired fibrinolysis of hypertriglyceridemia. Arterioscler Thromb Vasc Biol 1998; 18(1): 20-26. [DOI: 10.1161/01.ATV.18.1.20]; [PMID: 9445251]

460 Banfi C, Rise P, Mussoni L, Galli C, Tremoli E. Linoleic acid enhances the secretion of plasminogen activator inhibitor type 1 by HepG2 cells. J Lipid Res 1997; 38(5): 860-869]; [PMID: 9186904]

461 Boberg M, Pollare T, Siegbahn A, Vessby B. Supplementation with n-3 fatty acids reduces triglycerides but increases PAI-1 in non-insulin-dependent diabetes mellitus. Eur J Clin Invest 1992; 22(10): 645-650. [DOI: 10.1111/j.1365-2362.1992.tb01424.x]; [PMID: 1459169]

462 Eritsland J, Amesen H, Seljeflot I, Kierulf P. Long-term effects of n-3 fatty polyunsaturated fatty acids on haemostatic variables and bleeding episodes in patients with coronary artery disease. Blood Coagul Fibrinolysis 1995; 6(1): 17-22]; [PMID: 7795149]

463 Prandota J, Pankow-Prandota L, Kotecki L. Impaired activation of the fibrinolytic system in children with Henoch-Schönlein purpura: beneficial effect of hydrocortisone plus e-aminocaproic acid therapy on disappearance rate of cutaneous vasculitis and fibrinolysis. Am J Ther 2001; 8(1): 11-19. [PMID: 11304653]

464 Cierniewski CS. Progress in the knowledge on fibrinolysis regulation. Acta Haematol Pol 1994; 25(2 Suppl 2): 15-26]; [PMID: 7998470]

465 Besbas N, Erbay A, Saatci U, Ozdemir S, Bakkaloglu A, Ozen $\mathrm{S}$, et al. Thrombomodulin, tissue plasminogen activator and plasminogen activator inhibitor-1 in Henoch-Schönlein purpura. Clin Exp Rheumatol 1998; 16(1): 95-98]; [PMID: 9543574]

466 Kasza A, Kiss DL, Gopalan S, Xu W, Rydel RE, Koj A, et al. Mechanism of plasminogen activator inhibitor-1 regulation by oncostatin M and interleukin-1 in human astrocytes.J Neurochem 2002; 83(3): 696-703]; [PMID: 12390531]

467 Gallicchio M, Hufnagl P, Wojta J, Tipping P. IFN- $\gamma$ amma inhibits thrombin- and endotoxin-induced plasminogen activator inhibitor type 1 in human endothelial cells. J Immunol 1996; 157(6): 26102617]; [PMID: 8805664]

468 Massague J. The transforming growth factor-beta family. Annu Rev Cell Biol 1990; 6: 597-641. [DOI: 10.1146/annurev. cb.06.110190.003121]; [PMID: 2177343]

469 Roberts AB. Transforming growth factor-b: activity and efficacy in animal models of wound healing. Wound Repair Regen 1995; 3(4): 408-418. [DOI: 10.1046/j.1524-475X.1995.30405.x]; [PMID: 1503333]

470 Lawrence DA. Transforming growth factor-beta: a general review. Eur Cytokine Netw 1996; 7(3): 363-374]; [PMID: 8954178]

471 Samarakoon R, Higgins SP, Higgins CE, Higgins PJ. TGF- $\beta 1$ induced plasminogen activator inhibitor-1 expression in vascular smooth muscle cells requires pp60c-src/EGFRy845 and Rho/ ROCK signaling. J Mol Cell Cardiol 2008; 44(3): 527-538. [DOI: 10.1016/yjmcc.2007.12.0006]; [PMID: 18255094]

472 Barcellos-Hoff MH, Dix TA. Redox-mediated activation of latent transforming growth factor-beta. Mol Endocrinol 1996; 10(9): 1077-1083. [DOI: 10.1210/mend.10.9.8885242]; [PMID; 8885242]

473 Stroes E, Kastelein J, Cosentino F, Erkelens W, Wever R, Koomans $\mathrm{H}$, et al. Tetrahydrobiopterin restores endothelial function in hypercholesterolemia. J Clin Invest 1997; 99(1): 41-46. [DOI: 10.1172/JCI119131]; [PMID: 9011574]

474 Mäki-Petäjä KM, Day L, Cheriyan J, Hall FC, Östör AJK, Shenker N, et al. Tetrahydrobiopterin supplementation improves endothelial function but does not alter aortic stiffness in patients with rheumatoid arthritis. J Am Heart Assoc 2016; 5(2): e002762. [DOI: 10.1161/JAHA.115.002762]; [PMID: 26896473]

475 Landmesser U, Hornig B, Drexler H. Endothelial function. A critical determinant in atherosclerosis? Circulation 2004; 109(21 Suppl 1): II27-II33. [DOI: 10.1161/01. 
CIR.0000129501.88485.1f]; [PMID: 15173060]

476 Catani MV, Bernassola F, Rossi A, Melino G. Inhibition of clotting factor XIII activity by nitric oxide. Biochem Biophys Res Commun 1998; 249(1): 275-278. [DOI: 10.1006/bbrc.1998.9130]; [PMID: 9705871]

477 Lin Y, Chen Y, Zhu N, Zhao S, Fan J, Liu E. Hydrogen sulfide inhibits development of atherosclerosis through up-regulating protein S-nitrosylation. Biomed Pharmacol 2016; 83: 466-476. [DOI: 10.1016/j.biopha.2016.07.003]; [PMID: 27427853]

478 Van Galen KP, Tuinenburg A, Smeets EM, Schutgens RE. Von Willebrand factor deficiency and atherosclerosis. Blood Rev 2012; 26(5): 189-196. [DOI: 10.1016/j.blre.2012.05.002]; [PMID: 22721874]

479 Torisu T, Torisu K, Lee IH, Malide D, Combs CA, Wu XS, et al. Autophagy regulates endothelial cell processing, maturation and secretion of von Willebrand factor. Nat Med 2013; 19(10): 12811287. [DOI: 10.1036/nm.3288]; [PMID: 24056772]

480 Zwaginga JJ, Koomans HA, Sixma JJ, Rabelink AJ. Thrombus formation and platelet vessel wall interaction in the nephritic syndrome under flow conditions. J Clin Invest 1994; 93(1): 204211. [DOI: 10.1172/JCI116947]; [PMID: 8282789]

481 Booth NA, Simpson AJ, Croll A, Bennett B, Macgregor IR. Plasminogen activator inhibitor 1 (PAI-1) in plasma and platelets. Br J Haematol 1988; 70(3): 327-333. [DOI: 10.1111/j.13652141.1988.tb02490.x]; [PMID: 3264718]

482 Kruithof EK, Nicolosa G, Bachmann F. Plasminogen activator inhibitor 1: development of a radioimmunoassay and observations on its plasma concentration during venous occlusion and after platelet aggregation. Blood 1987; 70(5): 1645-1653]; [PMID: 3117137]

483 Simpson AJ, Booth NA, Moore NR, Bennett B. Distribution of plasminogen activator inhibitor (PAI-1) in tissues. $J$ Clin Pathol 1991; 44(2): 139-143]; [PMID: 1864986]

484 Watanabe T, Koba S. Roles of serotonin in atherothrombosis and related diseases. In: Traditional and Novel Risk Factors in Atherothrombosis (E. Gaxiola, ed.). In Tech, 2012; pp. 57-70.

485 Kawano H, Tsuji H, Nishimura H, Kimura S, Yano S, Ukimura $\mathrm{N}$, et al. Serotonin induces the expression of tissue factor and plasminogen activator inhibitor-1 in cultured rat aortic endothelial cells. Blood 2001; 97(6): 1697-1702. [DOI: 10.1182/blood. V97.6.1697]; [PMID: 11238110]

486 Katz MF, Farber HW, Dodds-Stitt Z, Cruikshank WW, Beer DJ. Serotonin-stimulated aortic endothelial cells secrete a novel T lymphocyte chemotactic and growth factor. J Leukoc Biol 1994; 55(5): 567-573]; [PMID: 7514199]

487 Pakala R, Willerson JT, Benedict CR. Mitogenic effect of serotonin on vascular endothelial cells. Circulation 1994; 90(4): 1919-1926. [DOI: 10.1161/01.CIR.90.4.1919]; [PMID: 7923680]

488 Pakala R, Willerson JT, Benedict CL. Effect of serotonin, thromboxane A2, and specific receptor antagonists on vascular smooth muscle cell proliferation. Circulation 1997; 96(7): 22802286. [DOI: 10.1161/01/CIR.96.7.2280]; [PMID: 9337201]

489 Ruiz-Perez MV, Sanchez-Jimenez F, Quesada AR, Medina MA. A re-evaluation of the mitogenic effect of serotonin on vascular smooth muscle cells. J Biol Regul Homeost Agents 2011; 25(1): 13-20]; [PMID: 21382269]

490 Suguro T, Watanabe T, Kanome T, Kodate S, Hirano T, Miyazaki A, et al. Serotonin acts as an up-regulator of acyl-coenzyme A: cholesterol acyltransferase-1 in human monocyte-macrophages. Atherosclerosis 2006; 186(2): 275-281. [DOI: 10.1016/ j.atherosclerosis.2005.08.007]; [PMID: 16157345]

491 Cook EH Jr, Leventhal BL, Heller W, Metz J, Wainwright M, Freedman DX. Autistic children and their first-degree relatives: relationship between serotonin and norepinephrine levels and intelligence. J Neuropsychiatr Clin Neurosci 1990; 2(3): 268-274. [DOI: 10.1176/jnp.2.3.268]; [PMID: 2136085]

492 Chugani DC. Serotonin in autism and pediatric epilepsies.
Mental Retard Dev Disabil Res Rev 2004 ; 10(2): 112-116. [DOI: 10.1002/mrdd.20021 [PMID : 15362166]

493 Cook EH Jr, Charak DA, Arida J, Spohn JA, Roizen NJ, Leventhal BL. Depressive and obsessive-compulsive symptoms in hyperserotonemic parents of children with autistic disorder. Psychiatr Res 1994; 52(1): 25-33. [DOI: 10.1016/01651781(94)90117-1]; [PMID: 8047619]

494 Piven J, Palmer P. Psychiatric disorder and the broad autism phenotype: evidence from a family study of multiple-evidence autism families. Am J Psychiat 1999; 156(4): 557-563. [DOI: 10.1176/ajp.156.4.557]; [PMID: 10200734]

495 Prandota J, Elleboudy NAF, Ismail KA, Zaki OK, Shehata HH. Increased seroprevalence of chronic toxoplasmosis in autistic children: special reference to the pathophysiology of IFN- $\gamma$ amma and NO overproduction. Int J Neurology Res 2015; 1(3): 102-122. [DOI: 10.17554/j.issn.2313-5611.2015.01.30]

496 Artwohl M, Graier WF, Roden M, Bischof M, Freudenthaler A, Waldhäusl W, et al. Diabetic LDL triggers apoptosis in vascular endothelial cells. Diabetes 2003; 52(5): 1240-1247. [DOI: 10.2337/diabetes.52.5.1240]; [PMID: 12716759]

497 Khan BV, Parthasarathy SS, Alexander RW, Medford RM. Modified low density lipoprotein and its constituents augment cytokine-activated vascular cell adhesion molecule-1 gene expression in human vascular endothelial cells. J Clin Invest 1995; 95(3): 1262-1270. [DOI: 10.1172/JCI117776]; [PMID: 7533787]

498 Lorenzi M, Cagliero E, Markey B, Henriksen T, Witztum JL, Sampietro T. Interaction of human endothelial cells with elevated glucose concentrations and native and glycosylated low density lipoproteins. Diabetologia 1984; 26(3): 218-222]; [PMID: 6714540]

499 Posch K, Simecek S, Wascher TC, Jürgens G, BaumagartnerParzer S, Kostner GM, et al. Glycated low-density lipoprotein attenuates shear stress-activated L-arginine uptake in endothelial cells. Diabetes 1999; 48(6): 1331-1337. [DOI: 10.2337/ diabetes.48.6.1331]; [PMID: 10342824]

500 Zimmermann R, Panzenböck U, Wintersperger A, Levak-Frank $\mathrm{S}$, Graier W, Glatter O, et al. Lipoprotein lipase mediates the uptake of glycated LDL in fibroblasts, endothelial cells, and macrophages. Diabetes 2001; 50(7): 1643-1653. [DOI: 10.2337/ diabetes.50.7.1643]; [PMID: 11423487]

501 Prandota J. T. gondii infection acquired prenatally and/or after birth may be responsible for development of both type 1 and 2 diabetes mellitus. J Diabetes Metab 2013; 4: 2. [DOI: 10.4172/2155-6156.1000241]

502 Maier JA, Malpuech-Brugere C, Zimowska W, Rayssiguier Y, Mazur A. Low magnesium promotes endothelial cell dysfunction: implications for atherosclerosis, inflammation and thrombosis. Biochim Biophys Acta 2004; 1689(1): 13-21. [DOI: 10.1016/ j.bbadis.2004.01.002]; [PMID: 15158909]

503 Vinchi F, Muckenthaler MU, Da Silva M, Balla G, Balla J, Jeney V. Atherogenesis and iron: from epidemiology to cellular level. Front Pharmacol 2014; 5: 94. [DOI: 10.3389/fphar.2014.00094]; [PMID: 24847266]

504 Sullivan JL. The iron paradigm of ischemic heart disease. Am Heart J 1981; 117(5): 1177-1188. [DOI: 10.1016/00028703(89)90887-9]; [PMID: 2653014]

505 Altamura S, Muckenthaler M. Iron toxicity in diseases of aging: Alzheimer's disease, Parkinson's disease and atherosclerosis. $J$ Alzheimer's Dis 2009; 16(4): 879-895. [DOI: 10.3233/JAD-20091010]; [PMID: 19387120]

506 Lee DW, Andersen JK. Iron elevations in the aging Parkinsonian brain: a consequence of impaired iron homeostasis?. J Neurochem 2010; 112(2): 332-339. [DOI: 10.1111/j.1471-4159.2009.06470. x]; [PMID: 20085612]

507 Sullivan JL. Iron in arterial plaque: modifiable risk factor for atherosclerosis. Biochim Biophys Acta 2009; 1790(7): 718-723. [DOI: 10.1016/j.bbagen.2008.06.005]; [PMID: 18619522] 
508 Kolodgie FD, Gold HK, Burke AP, Fowler DR, Kruth HS, Weber $\mathrm{DK}$, et al. Intraplaque hemorrhage and progression of coronary atheroma. $N$ Engl J Med 2003; 349(24): 2316-2325. [DOI: 10.1056/NEJMoa035655]; [PMID: 14668457]

509 Jeney V, Balla J, Yachie A, Varga Z, Vercellotti GM, Eaton JW, et al. Pro-oxidant and cytotoxic effects of circulating heme. Blood 2002; 100(3): 879-887. [DOI: 10.1182/blood.V100.3.879]; [PMID: 12130498]

510 Heinecke JW, Rosen H, Chait A. Iron and copper promote modification of low density lipoprotein by human arterial smooth muscle cells in culture. J Clin Invest 1984; 74(5): 1890-1894. [DOI: 10.1172/JCI111609]; [PMID: 6501577]

511 Stadler N, Lindner RA, Davies MJ. Direct detection and quantification of transition metal ions in human atherosclerotic plaques: evidence for the presence of elevated levels of iron and copper. Arterioscler Thromb Vasc Biol 2004; 24(5): 949954. [DOI: 10.1161/01.ATV.0000124892.90999.cb]; [PMID: 15001454]

512 Gerrity RG. The role of the monocyte in atherogenesis. Am J Pathol 1981; 103(2): 181-190]; [PMID: 7234961]

513 Crichton RR, Wilmet S, Legssyer R, Ward RJ. Molecular and cellular mechanisms of iron hemostasis and toxicity in mammal cells. J Inorg Biochem 2002; 91(1): 9-18. [DOI: 10.1016/S01620134(02)0046-0]; [PMID: 12121757]

514 Gkouvatsos K, Papanikolaou G, Pantopoulos K. Regulation of iron transport and the role of transferrin. Biochim Biophys Acta 2012; 1820(3): 188-202. [DOI: 10.1016/j.bbagen.2011.10.013]; [PMID: 22085723]

515 Weinberg ED. Iron loading and disease surveillance. Emerg Infect Dis 1999; 5(3): 346-52. [DOI: 10.3201/eid0503.990305]; [PMID: $10341171]$

516 McCord JM. Effects of positive iron status at a cellular level. Nutr Rev 1996; 54(3): 85-88. [DOI: 10.1111/j.1753-4887.1996.tb03876. x]; [PMID: 8935218]

517 Luck AN, Mason AB. Transferrin-mediated cellular iron delivery. Curr Top Membr 2012; 69: 3-35. [DOI: 10.1016/B978-0-12394390-3.00001-X]; [PMID: 23046645]

518 Reyes-Lopez M, Serrano-Luna J, Pina-Vazquez C, de la Garza M. Transferrin binding proteins as a means to obtain iron in parasitic protozoa. In: Binding Protein, Abdelmohsen K (ed.) InTech, 2012. [DOI: $10.5772 / 48288$ ]

519 Nagy E, Eaton JW, Jeney V, Soares MP, Varga Z, Galajda Z, et al. Red cells, hemoglobin, heme, iron, and atherogenesis. Arterioscler Thromb Vasc Biol 2010; 30(7): 1347-1353. [DOI: 10.1161/ ATVBAHA.110.206433]; [PMID: 20378845]

520 Silva G, Jeney V, Chora A, Larsen R, Balla I, Soares MP. Oxidized hemoglobin is an endogenous proinflammatory agonist that targets vascular endothelial cells. J Biol Chem 2009; 284(43): 2958229595. [DOI: 10.1074/jbc.M109.045344]; [PMID: 19700768]

521 Kurz T, Terman A, Brunk UT Autophagy, ageing and apoptosis: the role of oxidative stress and lysosomal iron. Arch Biochem Biophys 2007; 462(2): 220-230. [DOI: 10.1016/j.abb.2007.01.013]; [PMID: 17306211]

522 Martinet W, De Meyer GR. Autophagy in atherosclerosis. A cell survival and death phenomenon with therapeutic potential. Circ Res 2009; 104(3): 304-317. [DOI: 10.1161/ CIRCRESAHA.108.188318]; [PMID: 19213965]

523 Brunk UT, Jones CB, Sohal RS. A novel hypothesis of lipofuscinogenesis and cellular aging based on interactions between oxidative stress and autophagocytosis. Mutat Res 1992; 275(3-6): 395-403. [DOI: 10.1016/0921-8734(92)90042-N]; [PMID: 1383780

524 Lee FY, Lee TS, Pan CC, Huang AL, Chau LY. Colocalization of iron and ceroid in human atherosclerotic lesions. Atherosclerosis 1998; 138(2): 281-288. [DOI: 10.1016/S0021-9150(98)00033-1]; [PMID: 9690911]

525 Laliberte J, Carruthers VB. Host cell manipulation by the human pathogen Toxoplasma gondii. Cell Mol Life Sci 2008; 65(12): 1900-1915. [DOI: 10.1007/s00018-008-7556-x]; [PMID: 18327664]

526 Gail M, Gross U, Bohne W. Transferrin receptor induction in Toxoplasma gondii-infected HFF is associated with increased iron-responsive protein 1 activity and is mediated by secreted factors. Parasitol Res 2004; 94(3): 233-239. [DOI: 10.1007/ s00436-004-1209-2]; [PMID: 15349772]

527 Dimier IH, Bout DT. Interferon-gamma-activated primary enterocytes inhibit Toxoplasma gondii replication: a role for intracellular iron. Immunology 1998; 94(4): 488-495]; [PMID: 9767436]

28 Coppens I, Joiner KA. Host but not parasite cholesterol controls Toxoplasma cell entry by modulating organelle discharge. $\mathrm{Mol}$ Biol Cell 2003; 14(9): 3804-3820. [DOI: 10.1091/mbc.E02-120830]; [PMID: 12972565]

529 Pfefferkorn ER, Eckel M, Rebhun S. Interferon-gamma suppresses the growth of Toxoplasma gondii in human fibroblasts through starvation for tryptophan. Mol Biochem Parasitol 1986; 20(3): 215-224]; [PMID: 3093859]

530 Fox BA, Gigley JP, Bzik DJ. Toxoplasma gondii lacks the enzymes required for de novo arginine biosynthesis and arginine starvation triggers cyst formation. Int J Parasitol 2004; 34(3): 323-331. [DOI: 10.1016/j.ijpara.2003.12.001]; [PMID: 15003493]

531 Seabra SH, DaMatta RA, de Mello FG, de Souza W. Endogenous polyamine level in macrophages is sufficient to support growth of Toxoplasma gondii. J Parasitol 2004; 90(3): 455-460. [DOI: 10.1645/GE-179R]; [PMID: 15270085]

532 Chaudhary K, Darling JA, Fohl LM, Sullivan WJ Jr, Donald RG, Pfefferkorn ER, et al. Purine salvage pathways in the apicomplexan parasite Toxoplasma gondii. J Biol Chem 2004; 279(30): 31221-31227. [DOI: 10.1074/jbc.M404232200]; [PMID: 15140885]

533 Weinberg ED. The role of iron in protozoan and fungal infectious diseases. J Eukaryotic Microbiol 1999; 46(3): 231-238. [DOI: 10.1111/j.1550-7408.1999.tb05119.x]; [PMID: 10377984]

534 Ghio AJ, Piantadosi CA, Crumbliss AL. Hypothesis: iron chelation plays a vital role in neutrophilic inflammation. Biometals 1997; 10: 135-142]; [PMID: 9210296]

535 Chang H, Leblond CP. Origin, differentiation and renewal of the four main epithelial cell types in the mouse small intestine. I. Columnar cell. Am J Anat 1974; 141(4): 461-479. [DOI: 10.1002/ aja.1001410403]; [PMID: 4440632]

536 Bout D, Moretto M, Dimier-Poisson I, Gatel DB. Interaction between Toxoplasma gondii and enterocyte. Immunobiology 1999; 201: 225-228. [DOI: 10.1016/S0171-2985(99)80062-X]; [PMID: $10631574]$

537 Byrd TF, Horwitz MA. Interferon-gamma-activated human monocytes downregulate transferrin receptors and inhibit the intracellular multiplication of Legionella pneumophila by limiting the availability of iron. J Clin Invest 1989; 83(5): 1457-1465. [DOI: 10.1172/JCI114038]; [PMID: 2496141]

538 Lane TE, Wu-Hsieh B, Howard DH. Iron limitation and the gamma interferon-mediated antihistoplasma state of murine macrophages. Infect Immun 1991; 59(7): 2274-2278]; [PMID: 1904840]

539 Loo VG, Lalonde RG. Role of iron in intracellular growth of Trypanosoma cruzi. Infect Immun 1984; 45(3): 726-730]; [PMID: 6381312]

540 Pfefferkorn ER. Interferon-g blocks the growth of Toxoplasma gondii in human fibroblasts by inducing the host cells to degrade tryptophan. Proc Natl Acad Sci USA 1984; 81(3): 908-912]; [PMID: 6422465]

541 Halonen SK, Weiss LM. Investigation into the mechanism of gamma interferon-mediated inhibition of Toxoplasma gondii in murine astrocytes. Infect Immun 2000; 68(6): 3426-3430. [DOI: 10.1128/IAI.68.6.3426-3430.2000]; [PMID: 10816494]

542 Ghio AJ, Weinberg ED. Complications of TNF- $\alpha$ antagonists and 
iron homeostasis. Med Hypotheses 2012; 78(1): 333-5. [DOI: 10.1016/j.mehy.2011.09.035]; [PMID: 22000712]

543 Nairz M, Haschka D, Demetz E, Weiss G. Iron at the interface of immunity and infection. Front Pharmacol 2014; 5: 152. [DOI: 10.3389.fphar.2014.00152]; [PMID: 25076907]

544 Gudjoncik A, Guenancia M, Zeller M, Cottin Y, Vergely C, Rochette L. Iron, oxidative stress, and redox signaling in the cardiovascular system. Mol Nutr Food Res 2014; 58(8): 17211738. [DOI: 10.1002/mnfr.201400036]; [PMID: 24888568]

545 Reif DW, Simmons RD. Nitric oxide mediates iron release from ferritin. Arch Biochem Biophys 1990; 283(2): 537-541]; [PMID: 2177332]

546 Balla G, Jacob HS, Balla I, Rosenberg M, Nath K, Apple F, et al. Ferritin: a cytoprotective antioxidant strategem of endothelium. $J$ Biol Chem 1992; 267(25): 18148-18153]; [PMID: 1517245]

547 Weiss G, Werner-Felmayer G, Werner ER, Grünewald K, Wachter $\mathrm{H}$, Hentze MW. Iron regulates nitric oxide synthase activity by controlling nuclear transcription. $J$ Exp Med 1994; 180(3): 969976. [DOI: 10.1084/jem.180.3.969]; [PMID: 7520477]

548 Weinberg ED. Iron depletion: a defense against intracellular infection and neoplasia. Life Sci 1992; 50(18): 1289-1297]; [PMID: 1560730]

549 Casiglia E, Tikhonoff V. Inflammatory and coagulative markers of atherosclerosis. Eur Heart J 2007; 28(3): 271-273. [DOI: 10.1093/ eurheartj/eh1462]; [PMID: 17251260]

550 Wiseth R, Gunnes S, Madsen HO, Garred P. Multiple inflammatory markers in patients with significant coronary artery disease. Int $J$ Cardiol 2007; 118(1): 81-87. [DOI: 10.1016/j.ijcard.2006.07.005]; [PMID: 16935369]

551 Levula M, Oksala N, Airla N, Zeitlin R, Salenius JP, Järvinen $\mathrm{O}$, et al. Genes involved in systemic and arterial bed dependent atherosclerosis - Tampere Vascular study. PLoS ONE 2012; 7(4): e33787. [DOI: 10.1371/journal.pone.0033787]; [PMID: 22509262]

552 Weinberg ED. Human lactoferrin: a novel therapeutic with broad spectrum potential. J Pharm Pharmacol 2001; 53(10): 1303-1310. [DOI: 10.1211/0022357011777792]; [PMID: 11697537]

553 Dzitko K, Dziadek B, Dziadek J, Dlugonska H. Toxoplasma gondii: inhibition of the intracellular growth by human lactoferrin. Polish J Microbiol 2012; 56(1): 25-32]; [PMID: 17419186]

554 Kochan I. The role of iron in bacterial infections with special consideration of host-tubercle bacillus interaction. Curr Top Microbiol Immunol 1973; 60: 1-30]; [PMID: 4197776]

555 Aisen P, Enas C, Wesling-Resnick M. Chemistry and biology of eukaryotic iron metabolism. Int J Bioch Cell Biol 2001; 33(10): 940-949. [DOI: 10.1016/S1357-2725(01)00063-2]; [PMID: 11470229]

556 Wilson ME, Britigan BE. Iron acquisition by parasitic protozoa. Parasitol Today 1998; 14(9): 348-353. [DOI: 10.1016/S01694758(98)01294-0]; [PMID: 17040815]

557 Dziadek B, Dzitko K, Dlugonska H. Toxoplasma gondii binds human lactoferrin but not transferrin. Exp Parasitol 2005; 110(2): 165-167. [DOI: 10.1016/j.exppara.2005.03.019]; [PMID: 15888300]

558 Suzuki YA, Lopez V, Lönnerdal B. Mammalian lactoferrin receptors: structure and function. Cell Mol Life Sci 2005; 62(22): 2560-2575. [DOI: 10.1007/s00018-005-5371-1]; [PMID: 16261254]

559 Lima MF, Kierszenbaum F. Lactoferrin effects of phagocytic cell function. I. Increased uptake and killing of an intracellular parasite by murine macrophages and human monocytes. $J$ Immunol 1985; 134(6): 4176-4183]; [PMID: 3886800]

560 Lima MF, Kierszenbaum F. Lactoferrin effects of phagocytic cell function. II. The presence of iron is required for the lactoferrin molecule to stimulate intracellular killing by macrophages but not enhance the uptake of particles and microorganisms. J Immunol 1987; 139(5): 1647-1651]; [PMID: 3114372]
561 Dziadek B, Dzitko K, Dlugonska H. Identification of Toxoplasma gondii proteins binding human lactoferrin: a new aspect of rhoptry proteins function. Exp Parasitol 2007; 115(3): 277-282. [DOI: 10.1016/j.exppara.2006.09.012]; [PMID: 17069806]

562 Safaeian L, Javanmard HS, Mollanoori Y, Dana N. Cytoprotective and antioxidant effects of human lactoferrin against $\mathrm{H} 202$-induced oxidative stress in human umbilical vein endothelial cells. $A d v$ Biomed Res 2015; 4: 188. [DOI: 10.4103/2277-9175.164010]; [PMID: 26605227]

563 Puddu P, Valenti P, Gessani S. Immunomodulatory effects of lactoferrin on antigen presenting cells. Biochimie 2009; 91: 11-18. [DOI: 10.1016/j.biochi.2008.05.005]; [PMID: 18539153]

564 Siqueiros-Cendon T, Arevalo-Gallegos S, Iglesias-Figueroa BF, Garcia-Montoya IA, Salazar-Martinez J, Rascon-Cruz Q. Immunomodulatory effects of lactoferrin. Acta Pharmacol Sin 2014; 35(5): 557-566. [DOI: 10.1038/aps.2013.200]; [PMID: 24786230]

565 Fernandez-Real JM, Garcia-Fuentes E, Moreno-Navarrete JM, Murri-Pierri M, Garrido-Sanchez L, Ricart W, et al. Fat overload induces changes in circulating lactoferrin that are associated with postprandial lipemia and oxidative stress in severly obese subjects. Obesity (Silver Spring) 2010; 18(3): 482-488. [DOI: 10.1038/ oby.2009.266]; [PMID: 19696758]

566 Takeuchi T, Shimizu H, Ando K, Harada E. Bovine lactoferrin reduces plasma triacylglycerol and NEFA accompanied by decreased hepatic cholesterol and triacylglycerol contents in rodents. Br J Nutr 2004; 91(4): 533-538. [DOI: 10.1079/ BJN20041090]; [PMID: 15035680]

567 Libby P, Ridker PM, Hansson GK. Inflammation in atherosclerosis: from pathophysiology to practice. J Am Coll Cardiol 2009; 54(23): 2129-2138. [DOI: 10.1016/j.jacc.2009.09.009]; [PMID: 19942084]

568 Grainger DJ, Kemp PR, Metcalf JC, Liu AC, Lawn RM, Williams $\mathrm{NR}$, et al. The serum concentration of active transforming growth beta is severely depressed in advanced atherosclerosis. Nat Med 1995; 1(1): 74-79]; [PMID: 7584958]

569 Grainger DJ. TGF- $\beta$ and atherosclerosis in man. Cardiovasc Res 2007; 74(2): 213-222. [DOI: 10.1016/j.cardiores.2007.02.022]; [PMID: 17382916]

570 Blann AD, Wang JM, Wilson PB, Kumar S. Serum levels of TGFBeta receptor are increased in atherosclerosis. Atherosclerosis 1996; 120(1-2): 221-226. [DOI: 10.1016/0021-9150(95)05713-7]; [PMID: 8645363]

571 Robertson AK, Rudling M, Zhou X, Gorelik L, Flavell RA, Hansson GK. Disruption of TGF- $\beta$ signaling accelerates atherosclerosis. $J$ Clin Invest 2003; 112(9): 1342-1350. [DOI: 10.1172/JCI18607]; [PMID: 14568988]

572 Ait-Oufella H, Salomon BL, Potteaux S, Robertson AK, Gourdy $\mathrm{P}$, Zoll J, et al. Natural regulatory T cells control the development of atherosclerosis in mice. Nat Med 2006; 12(2): 178-180. [DOI: 10.1036/nm1343]; [PMID: 16462800]

573 Mallat Z, Gojova A, Marchiol-Fournigault C, Esposito B, Kamate $\mathrm{C}$, Merval $\mathrm{R}$, et al. Inhibition of transforming growth factor-b signaling accelerates atherosclerosis and induces an unstable plaque phenotype in mice. Circ Res 2001; 89(10): 930-934. [DOI: 10.1161/hh2201.099415]; [PMID: 11701621]

574 Lievens D, Habets KL, Robertson A-K, Laouar Y, Winkels H, Rademakers $\mathrm{D}$, et al. Abrogated transforming growth factor beta receptor II (TGFbRII) signaling in dendritic cells promotes immune reactivity of $\mathrm{T}$ cells resulting in enhanced atherosclerosis. Eur Heart J 2013; 34(48): 3717-3727. [DOI: 10.1093/eurheartj/ ehs106]; [PMID: 22613345]

575 Letterio JJ, Roberts AB. Regulation of immune responses by TGF- $\beta$. Ann Rev Immunol 1998; 16: 137-161. [DOI: 10.1146/ annurev.immunol.16.1.137]; [PMID: 9597127]

576 Wahl SM. Transforming growth factor beta (TGF- $\beta$ ) in inflammation: a cause and a cure. J Clin Immunol 1992; 12(2): 61- 
74. [PMID: 1313827]

577 Horwitz DA, Gray JD, Ohtsuka K, Hirokawa M, Takahashi T. The immunoregulatory effects of NK cells: the role of TGF- $\beta$ and implications for autoimmunity. Immunol Today 1997; 18(11): 538542. [DOI: 10.1016/S0167-5699(97)01149-3]; [PMID: 9386350]

578 Hansson GK. Inflammatory mechanisms in atherosclerosis. $J$ Thromb Haemost 2009; 7(Suppl. 1): 328-331. [DOI: 10.1111/ j.1538-7836.2009.03416.x]

579 Omer FM, Kurtzhals JA, Riley EM. Maintaining the immunological balance in parasitic infections: a role for TGF- $\beta$ ? Parasitol Today 2000; 16(1): 18-23. [DOI: org/10.1016/S01694758(99)01562-8]; [PMID: 10637583]

580 Robertson AK, Hansson GK. T cells in atherogenesis: for better or for worse?. Arterioscler Thromb Vasc Biol 2006; 26(11): 24212432. [DOI: 10.1161/01.ATV.0000245830.29764.84]; [PMID: 16973967]

581 Hansson GK, Libby P. The immune response in atherosclerosis: a double-edged sword. Nat Rev Immunol 2006; 6(7): 508-519. [DOI: 10.1038/nri1882]; [PMID: 16778830]

582 Tonkin DR, Haskins K. Regulatory T cells enter the pancreas during suppression of type 1 diabetes and inhibit effector $\mathrm{T}$ cells and macrophages in a TGF- $\beta$-dependent manner. Eur J Immunol 2009; 39(5): 1313-1322. [DOI: 10.1002/eji.200838916]; [PMID: 19404982]

583 Chen S, Hong SW, Iglesias-de la Cruz MC, Isono M, Casaretto A, Ziyadeh FN. The key role of the transforming growth factor-beta system in the pathogenesis of diabetic nephropathy. Renal Failure 2001; 23(3-4): 471-481. [DOI: 10.1081/JDI-100104730]; [PMID: 11499562]

584 Chen S, Jim B, Ziyadeh FN. Diabetic nephropathy and transforming growth factor-beta: transforming our view of glomerulosclerosis and fibrosis build-up. Semin Nephrol 2003; 23(6): 532-543. [DOI: 10.1053/S0270-9295(03)00132-3]; [PMID: $14631561]$

585 Park K, Ryu SB, Park YI, Ahn K, Lee SN, Nam JH. Diabetes mellitus induces vaginal tissue fibrosis by TGF- $\beta$ eta 1 expression in the rat model. $J$ Sex Marital Ther 2001; 27(5): 577-587. [DOI: 10.1080/713846811]; [PMID: 11554221]

586 Boucher BJ. Vitamin D insufficiency and diabetes risk. Curr Drug Targets 2011; 12(1): 61-87. [DOI: 10.2174/138945011793591653]; [PMID: 20795936]

587 Suzumura A, Sawada M, Yamamoto H, Marunouchi T. Transforming growth factor-beta suppresses activation and proliferation of microglia in vitro. J Immunol 1993; 151(4): 21502158]; [PMID: 8345199]

588 Yener S, Demir T, Akinci B, Bayraktar F, Kebapcilar L, Ozcan MA, et al. Transforming growth factor-beta 1 levels in women with prior history of gestational diabetes mellitus. Diabetes Res Clin Practice 2007; 76(2): 193-198. [DOI: 10.1016/ j.diabres.2006.08.014]; [PMID: 17014924]

589 Shrikant P, Lee SJ, Kalvakolanu I, Ransohoff RM, Benveniste EN. Stimulus-s[ecific inhibition of intracellular adhesion molecule-1 gene expression by TGF-ßeta. J Immunol 1996; 157(2): 892-900]; [PMID: 8752943]

590 Winkler MK, Beveniste EN. Transforming growth factor-beta inhibition of cytokine-induced vascular cell adhesion molecule-1 expression in human astrocytes. Glia 1998; 22(2): 171-179]; [PMID: 9537837]

591 Hunter CA, Bermudez L, Beernink H, Waegell W, Remington JS. Transforming growth factor-beta inhibits interleukin 12-induced production of interferon-gamma by natural killer cells: a role for transforming growth factor-beta in the regulation of $\mathrm{T}$ cellindependent resistance to Toxoplasma gondii. Eur J Immunol 1995; 25(4): 994-1000. [DOI: 10.1002/eji.1830250420]; [PMID: 7737303]

592 Langermans JA, Nibbering PH, Van Vuren-Van der Hulst ME, Van Furth R. Transforming growth factor-beta suppresses interferon- gamma-induced toxoplasmastatic activity in murine macrophages by inhibition of tumour necrosis factor-alpha production. Parasite Immunol 2001; 23(4): 169-175. [DOI: 10.1046/j.13653024.2001.00371.x]; [PMID: 11298293]

593 Schlüter D, Bertsch D, Frei K, Hübers SB, Wiestler OD, Hof H, et al. Interferon-gamma antagonizes transforming growth factorbeta2-mediated immunosuppression in murine Toxoplasma encephalitis. J Neuroimmunol 1998; 81(1-2): 38-48. [DOI: 10.1016/S0165-5728(97)00156-2]; [PMID: 9521604]

594 Buzoni-Gatel D, Debbabi H, Mennechet FJ, Martin V, Lepage AC, Schwartzman JD, et al. Murine ileitis after intracellular parasite infection is controlled by TGF- $\beta$ eta-producing intraepithelial lymphocytes. Gastroenterology 2001; 120(4): 914-924. [DOI: 10.1053/gast.2001.22432a]; [PMID: 11231945]

595 Denkers EY, Gazzinelli RT. Regulation and function of T-cellmediated immunity during Toxoplasma gondii infection. Clin Microbiol Rev 1998; 11(4): 569-588]; [PMID: 9767056]

596 Hughes HP. Oxidative killing of intracellular parasites mediated by macrophages. Parasitol Today 1988; 4(12): 340-347. [DOI: 10.1016/0169-4758(88)90003-8]; [PMID: 15463026]

597 Ding AH, Nathan CF, Stuehr DJ. Release of reactive nitrogen Intermediates and reactive oxygen intermediates from mouse peritoneal macrophages. J Immunol 1988; 141(7): 2407-2412]; [PMID: 3139757$]$

598 Pfefferkorn ER, Rebhun S, Eckel M. Characterization of an indoleamine 2,3-dioxygenase induced by gamma-interferon in cultured human fibroblasts. J Interferon Res 1986; 6(3): 267-279. [DOI: 10.1089/jir.1986.6.267]; [PMID: 2427623]

599 Barbosa BF, Silva DAO, Costa IN, Mineo JR, Ferro EA. BeWo trophoblast cell susceptibility to Toxoplasma gondii is increased by interferon-g, interleukin-10 and transforming factor-b1. Clin Exp Immunol 2008; 151(3): 536-545. [DOI: 10.1111/j.13652249.2007.03583.x]; [PMID: 18234060]

600 Oliveira JG, Silva NM, Santos AA, Souza MA, Ferreira GL, Mineo JR, et al. BeWo trophoblasts are unable to control replication of Toxoplasma gondii, even in the presence of exogenous IFNramma. Placenta 2006; 27(6-7): 691-698. [DOI: 10.1016/ j.placenta.2005.06.006]; [PMID: 16122791]

601 Malipiero U, Koedel U, Pfister W, Fontana A. Bacterial meningitis: the role of transforming growth factor-beta in innate immunity and secondary damage. Neurodegener Dis 2007; 4(1): 43-50. [DOI: 10.1159/000100358]; [PMID: 17429218]

602 Gaddi PJ, Yap GS. Cytokine regulation of immunopathology in toxoplasmosis. Immunol Cell Biol 2007; 85(2): 155-159. [DOI: 10.1038/sj.icb.7100038]; [PMID: 17228318]

603 Luangsay S, Kasper LH, Rachinel N, Minns LA, Mennechet FJD, Vandewalle A, et al. CCR5 mediates specific migration of Toxoplasma gondii-primed CD8+ lymphocytes to inflammatory epithelial cells. Gastroenterology 2003; 125(2): 491-500. [DOI: 10.1016/S0016-5085(03)00903-X]; [PMID: 12891552]

604 Connor TB, Roberts AB, Sporn MB, Danielpour D, Dart LL, Michels RG, et al. Correlation of fibrosis and transforming growth factor-b type 2 levels in the eye. J Clin Invest 1989; 83(5): 16611666. [DOI: 10.1172/JCI114065]; [PMID: 2708527]

605 Sarciron ME, Gherardi A. Cytokines involved in Toxoplasmic encephalitis. Scan J Immunol 2000; 52(6): 534-543. [DOI: 10.1111/j.1365-3083.2000.00817.x]; [PMID: 11119257]

606 Lutgens E, Gijbels M, Smook M, Heeringa P, Gotwals P, Koteliansky WE, et al. Transforming growth factor TGF- $\beta$ mediates balance between inflammation and fibrosis during plaque formation. Arterioscler Thromb Vasc Biol 2002; 22(6): 975-982. [DOI: 10.1161/01.ATV.0000019729.39500.2F]; [PMID: 12067907]

607 Seabra SH, de Souza W, Damatta RA. Toxoplasma gondii exposes phosphatidylserine inducing a TGF- $\beta$ etal autocrine effect orchestrating macrophage evasion. Biochem Biophys Res Commun 2004; 324(2): 744-752. [DOI: 10.1016/j.bbrc.2004.09.113]; 
[PMID: 15474490]

608 Bot I, Biessen EA. Mast cells in atherosclerosis. Thromb Haemost 2011; 106: 820-826. [DOI: 10.1160/TH11-05-0291]; [PMID: 21866302]

609 Kaartinen M, Penttilä A, Kovanen PT. Accumulation of activated mast cells in the shoulder region of human coronary atheroma, the predilection site of atheromatous rupture. Circulation 1994; 90(4): 1669-1678. [DOI: 10.1161/01.CIR.90.4.1669]; [PMID: 7923651]

610 Kovanen PT. The mast cell - a potential link between inflammation and cellular cholesterol deposition in atherogenesis. Eur Heart $J$ 1993; 14(Suppl K): 105-117]; [PMID: 8131778]

611 Galli SJ, Nakae S, Tsai M. Mast cells in the development of adaptive immune responses. Nat Immunol 2005; 6(2): 135-142. [DOI: 10.1038/ni1158]; [PMID: 15662442]

612 Theoharides TC, Alysandratos KD, Angelidou A, Delivanis DA, Sismanopoulos N, Zhang B, et al. Mast cells and inflammation. Biochim Biophys Acta 2010; 1822(1): 21-33. [DOI: 10.1016/ j.bbadis.2010.12.014]; [PMID: 21185371]

613 Kelley JL, Chi DS, Abou-Auda W, Smith JK, Kirshnaswamy G. The molecular role of mast cells in atherosclerotic cardiovascular disease. Mol Med Today 2000; 6(8): 304-308]; [PMID: 10904247]

614 Zhang J, Alcaide P, Liu L, Sun J, He A, Luscinskas FW, et al. Regulation of endothelial cell adhesion molecule expression by mast cells, macrophages, and neutrophils. PLOS ONE 2011; 6(1): e14525. [DOI: 10.1371/journal.pone.0014525]; [PMID: 21264293]

615 Kovanen PT. Mast cells in human fatty streaks and atheromas: implications for intimal lipid accumulation. Curr Opin Lipiodol 1996; 7(5): 281-286]; [PMID: 8937517]

616 Jeziorska M, McCollum C, Woolley DE. Mast cell distribution, activation, and phenotype in atherosclerotic lesions of human carotid arteries. J Pathol 1997; 182(1): 115-122. [DOI: 10.1002/ (SICI)1096-9896(199705182: 1<115: AID-PATH806>3.0.C): 2-9]; [PMID: 9227350]

617 Kokkonen JO, Kovanen PT. Stimulation of mast cells leads to cholesterol accumulation in macrophages in vitro by a mast cellgranule-mediated uptake of low density lipoprotein. Proc Natl Acad Sci USA 1987; 84(8): 2287-2291]; [PMID: 3470793]

618 Kokkonen JO, Kovanen PT. Low-density-lipoprotein binding by mast-cell granules. Demonstration of binding of apiolipoprotein B to heparin proteoglycan of exocytosed granules. Biochem J 1987; 241(2): 583-589. [DOI: 10.1042/bj2410583]; [PMID: 3593208]

619 Steinberg D, Parthasarathy S, Carew TE, Khoo JC, Witztum JL. Beyond cholesterol. Modifications of low-density lipoprotein that increase its atherogenicity. $N$ Engl J Med 1989; 320(14): 915-924. [DOI: 10.1056/NEJM198904063201407]; [PMID: 2648148]

620 Lee M, Lindstedt LK, Kovanen PT. Mast cell-mediated inhibition of reverse cholesterol transport. Arterioscler Thromb Vasc Biol 1992; 12(11): 1329-1335. [DOI: 10.116101.ATV.12.11.1329]; [PMID: 1420092]

621 Kovanen PT. Role of mast cells in atherosclerosis. Chem Immunol 1995; 62: 132-170]; [PMID: 7546279]

622 Yeong P, Ning Y, Xu Y, Yin L. Tryptase promotes human monocyte-derived macrophage foam cell formation by suppressing LXRalpha activation. Biochim Biophys Acta 2010; 1801(5): 567576. [DOI: 10.1016/bbalip.2010.01.011]; [PMID: 20139031]

623 Wilson CB, Remington JS. Activity of human blood leukocytes against Toxoplasma gondii. J Infect Dis 1979; 140(6): 890-895]; [PMID: 541523]

624 Yong EC, Chi EY, Henderson WR Jr. Toxoplasma gondii alters eicosanoid release by human mononuclear phagocytes: role of leukotrienes in interferon g-induced antitoxoplasma activity. $J$ Exp Med 1994; 180(5): 1637-1648. [DOI: 10.1084/jem.180.5.1637]; [PMID: 7964451]

625 Henderson WR Jr, Chi EY. The importance of leukotrienes in mast cell-mediated Toxoplasma gondii cytotoxicity. J Infect Dis 1997; 177(5): 1437-1443]; [PMID: 9593043]
626 Cruz A, Mendes EA, de Andrade MVM, Nascimento VC, Cartelle $\mathrm{CT}$, Arantes RM, et al. Mast cells are crucial in the resistance against Toxoplasma gondii oral infection. Eur J Immunol 2014; 44(10): 2949-2954. [DOI: 10.1002/eji.201344135]; [PMID: 25091816]

627 Dlugonska H. Toxoplasma gondii and mast cells. Ann Parasitol 2014; 60(4): 235-238]; [PMID: 25706419]

628 Huang B, Huang S, Chen Y, Zheng H, Shen J, et al. Mast cells modulate acute toxoplasmosis in murine models. PLOS ONE 2013; 8(10): e77327. [DOI: 10.1371/journal.pone.0077327]; [PMID: 24146978]

629 Ferreira GL, Mineo JR, Oliveira JG, Ferro EA, Souza A, Santos AA. Toxoplasma gondii and mast cell interactions in vivo and in vitro: experimental infection approaches in Calomys callossus (Rodentia, Cricetidae). Microbes Infect 2004; 6(2): 172-181. [DOI: 10.1016/j.micinf.2003.11.007]; [PMID: 14998515]

630 Smith NL, Abi Abdallah DS, Butcher BA, Denkers EY, Baird B, Holowka D. Toxoplasma gondii inhibits mast cell degranulation by suppressing phospholipase $\mathrm{Cg}$-mediated $\mathrm{Ca}^{2+}$ mobilization. Front Microbiol 2013; 4: 179. [DOI: 10.3389/fmicb.2013.00179]; [PMID: 23847603]

631 Theoharides TC, Kempuraj D, Tagen M, Conti P, Kalogeromitros D. Differential release of mast cell mediators and the pathogenesis of inflammation. Immunol Rev 2007; 217: 65-78. [DOI: 10.1111/ j.1600-065X.2007.00519.x]; [PMID: 174998052]

632 Kandere-Grzybowska K, Letourneau R, Kempuraj D, Donelan J, Poplawski S, Boucher W, et al. IL-1 induces vesicular secretion of IL-6 without degranulation from human mast cells. J Immunol 2003; 171(9): 4830-4836

633 Ban Y, Watanabe T, Miyazaki A, Nakano Y, Tobe T, Idei T, et al. Impact of increased plasma serotonin levels and acrotid atherosclerosis on vascular dementia. Atherosclerosis 2007; 195(1): 153-159. [DOI: 10.1016/j.atherosclerosis.2006.09.005]; [PMID: 17049533]

634 Lorenowicz MJ, van Gils J, de Boer M, Hordijk PL, FernandezBorja M. Epac1-Rap1 signaling regulates monocyte adhesion and chemotaxis. J Leukoc Biol 2006; 80(6): 1542-1552. [DOI: 10.1189/jlb.0506357]; [PMID: 16940330]

635 Aviram M, Fuhrman B, Maor I, Brook GJ. Serotonin increases macrophage uptake of oxidized low density lipoprotein. Eur $J$ Clin Chem Clin Biochem 1992; 30(2): 55-61]; [PMID: 1581411]

636 Ito T, Ikeda U, Shimpo M, Yamamoto K, Shimada K. Serotonin increases interleukin-6 synthesis in human vascular smooth muscle cells. Circulation 2000; 102(20): 2522-2527. [DOI: 10.1161/01.CIR.102.20.2522]; [PMID: 11076827]

637 Yong EC, Chi EY, Fritsche TR, Henderson WR Jr. Human platelet-mediated cytotoxicity against Toxoplasma gondii: role of thromboxane. J Exp Med 1991; 173(1): 65-78. [DOI: 10.1084/ jem.173.1.65]; [PMID: 1898664]

638 Henderson WR, Rashed M, Yong EC, Frische TR, Chiang GK. Toxoplasma gondii stimulates the release of 13- and 9-hydroxyoctadecadienoic acids by human platelets. Biochemistry 1992; 31: 5356-5362

639 Henderson WR, Chi EY. Cytotoxic activity of 13-hydroxyoctadecadienoic acid against Toxoplasma gondii. Parasitology 1992; 105(Pt 3): 343-347]; [PMID: 1451675]

640 Shamseddin J, Akhlaghi L, Razmjou E, Shojaee S, Monavari SHR, Tajik N, et al. Conjugated linoleic acid stimulates apoptosis in $\mathrm{RH}$ and Teheran strains of Toxoplasma gondii, in vitro. Iran $J$ Parasitol 2015; 10(2): 238-244]; [PMID: 26246821]

641 Sarraf P, Frederich RC, Turner EM, Ma G, Jaskowiak NT, Rivet DJ $3^{\text {rd }}$, et al. Multiple cytokines and acute inflammation raise mouse leptin levels: potential role in inflammatory anorexia. J Exp Med 1997; 185(1): 171-175. [DOI: 10.1084/jem.185.1.171]; [PMID: 8996253]

642 Havel PJ. Peripheral signals conveying metabolic information to the brain: short-term and long-term regulation of food intake and 
energy homeostasis. Exp Biol Med (Maywood) 2001; 226(11): 963-977]; [PMID: 11743131]

643 La Cava A, Matarese G. The weight of leptin in immunity. Nat Rev Immunol 2008 ; 4(5): 371-379. [DOI: 10.1038/nri1350]; [PMID: 15122202]

644 Iikuni N, Lam QL, Lu L, Matarese G, La Cava A. Leptin and inflammation. Curr Immunol Rev 2008; 4(2): 70-79. [DOI: 10.2174/1573395087843255046]; [PMID: 20198122]

645 van Dielen FM, van’t Veer C, Schols AM, Soeters PB, Buurman WA, Greve JW. Increased leptin concentrations correlate with increased concentrations of inflammatory markers in morbidly obese individuals. Int J Obese Relat Metab Disord 2001; 25(12): 1759-1766. [DOI: 10.1038/sj.ijo.0801825]; [PMID: 11781755]

646 Matarese G. Leptin and the immune system: how nutritional status influences the immune response. Eur Cytokine Netw 2000; 11(1): 7-14]; [PMID: 10705294]

647 Kim KY, Kim JK, Han SH, Lim JS, Kim KI, Cho DH, et al. Adiponectin is a negative regulator of NK cell cytotoxicity. J Immunol 2006; 176(10): 5958-5964. [DOI: 10.4049/ jimmunol.176.10.5958]; [PMID: 16670304]

648 Fujita Y, Murakami M, Ogawa Y, Masuzaki H, Tanaka M, Ozaki $\mathrm{S}$, et al. Leptin inhibits stress-induced apoptosis of T lymphocytes. Clin Exp Immunol 2002; 128(1): 21-26. [DOI: 10.1046/j.13652249.2002.01797.x]; [PMID: 11982586]

649 Park HY, Kwon HM, Lim HJ, Hong BK, Lee JY, Park BE, et al. Potential role of leptin in angiogenesis: leptin induces endothelial cell proliferation and expression of matrix metalloproteinases in vivo and in vitro. Exp Mol Med 2001; 33(2): 95-102. [DOI: 10.1038/emm.2001.17]; [PMID: 11460888]

50 Jones SA. Directing transition from innate to acquired immunity: defining a role of IL-6. J Immunol 2005; 175(6): 3463-3468. [DOI: 10.4049/jimmunol.175.6.3463]; [PMID: 16148087]

651 Yokota K, Miyazaki T, Hirano M, Akiyama Y, Mimura T. Simvastatin inhibits production of interleukin 6 (IL-6) and IL-8 and cell proliferation nduced by tumor necrosis factor-a in fibroblast-like synoviocytes from patients with rheumatoid arthritis. J Rheumatol 2006; 33(3): 463-471]; [PMID: 16511915]

652 Reeves GM, Mazaheri S, Snitker S, Langenberg P, Giegling I, Hartmann AM, et al. A positive association between T. gondii seropositivity and obesity. Front Publ Health 2013; 1: 73. [DOI: 10.3389/fpubh.2013.00073]; [PMID: 24400300]

653 Baltaci AK, Mogulkoc R. Plasma leptin levels in rats with induced Toxoplasma gondii infection. Bratisl Lek Listy 2012; 113(2): 6769. [DOI: 10.4149/BLL 2012 016]; [PMID: 22394033]

654 Aygun AD, Gungor S, Ustundag B, Gurgoze MK, Sen Y. Proinflammatory cytokines and leptin are increased in serum of prepubertal obese children. Mediators Inflamm 2005; 3: 180-183. [DOI: 10.1155/MI.2005.180]; [PMID: 16106106]

655 Pollmächer T, Schuld A, Kraus T, Haack M, Hinze-Selch D. On the clinical relevance of clozapine-triggered release of cytokines and soluble cytokine-receptors. Fortschr Neurol Psychiatr 2001; 69 Suppl 2: 65-74. [DOI: 10.1055/s-2001-16533]; [PMID: 11533853]

656 Asensio CS, Arsenijevic D, Lehr L, Giacobino JP, Muzzin P, Rohner-Jeanrenaud F. Effects of leptin on energy metabolism in beta-less mice. Int J Obesity (London) 2008; 32(6): 936-942. [DOI: 10.1038/ijo.2008.13]; [PMID: 18283283]

657 Arsenijevic D, Girardier L, Seydoux J, Chang HR, Dulloo AG. Altered energy balance and cytokine expression in a murine model of chronic infection with Toxoplasma gondii. Am J Physiol 1997; 272(5 Pt 1): E908-E917]; [PMID: 9176193]

658 Arsenijevic D, De Bilbao F, Giannakopoulos P, Girardier L, Samec $\mathrm{S}$, Richard D. A role of interferon-gamma in the hypermetabolic response to murine toxoplasmosis. Eur Cytokine Net 2001; 12(3): 518-527]; [PMID: 11566633]

659 Matthys P, Billiau A. Cytokines and cachexia. Nutrition 1997; 13(9): 763-770. [DOI: 10.1016/S0899-9007(97)00185-8]; [PMID:
9290087]

660 Plata-Salaman CR. Central nervous system mechanisms contributing to the cachexia-anorexia syndrome. Nutrition 2000; 16(10): 1009-1002. [DOI: 10.1016/S0899-9007(00)00413-5]; [PMID: 11054608]

661 Kelley T, Yang W, Chen CS, Reynolds K, He J. Global burden of obesity in 2005 and projections to 2030. Int J Obes 2008; 32(9): 1431-1437. [DOI: 10.1038/ijo.2008.102]; [PMID: 18607383]

662 Reiser J, Adair B, Rheinheckel T. Specialized roles for cysteine cathepsins in health and disease. J Clin Invest 2010; 120(10): 3421-3431. [DOI: 10.1172/JCI42918]; [PMID: 20921628]

663 Honey K, Rudensky AY. Lysosomal cysteine proteases regulate antigen presentation. Nat Rev Immunol 2003; 3(6): 472-482. [DOI: 10.1038/nri1110]; [PMID: 12776207]

664 Funkelstein L, Toneff T, Mosier C, Hwang SR, Beuschlein F, Lichtenauer UD, et al. Major role of cathepsin L for producing the peptide hormones ACTH, beta-endorphin, and alpha-MSH, illustrated by protease gene knockout and expression. J Biol Chem 2008; 283(51): 35652-35659. [DOI: 10.1074/jbc.M709010200]; [PMID: 18849346]

665 Deal C. Potential new targets for osteoporosis. Nat Clin Pract Rheumatol 2009; 5(1): 20-27. [DOI: 10.1038/ncprheum0977]; [PMID: 19098925]

666 Riese RJ, Mitchell RN, Villadangos JA, Shi GP, Palmer JT, Karp $\mathrm{ER}$, et al. Cathepsin $\mathrm{S}$ activity regulates antigen presentation and immunity. J Clin Invest 1998; 101(11): 2351-2363. [DOI: 10.1172/JCI1158]; [PMID: 9616206]

667 Shi GP, Villadangos JA, Dranoff G, Small C, Gu L, Haley KJ, et al. Cathepsin S required for normal MHC class II peptide loading and germinal center development. Immunity 1999; 10(2): 197-206. [DOI: 10.1016/S1074-7613(00)80020-5]; [PMID: 10072072]

668 Shi GP, Bryant RA, Riese RJ, Verhelst S, Driessen C, Li Z, et al. Role for cathepsin $\mathrm{F}$ in invariant chain processing and major histocompatibility complex class II peptide loading by macrophages. J Exp Med 2000; 191(7): 1177-1186. [DOI: 10.1084/jem.191.7.1177]; [PMID: 10748235]

669 Li W, Yuan XM. Increased expression and translocation of lysosomal cathepsins contribute to macrophage apoptosis in atherogenesis. Ann NY Acad Sci 2004; 1030: 427-433. [DOI: 10.1196/annals.1329.053]; [PMID: 15659826]

670 De Nooijer R, Bot I, von der Thüsen JH, Leeuwenburgh MA, Overkleeft HS, Kraaijeveld AO, et al. Leukocyte cathepsin S is a potent regulator of both cell and matrix turnover in advanced atherosclerosis. Arterioscler Thromb Vasc Biol 2009; 29(2): 188194. [DOI: 10.1161/ATVBAHA.108.181578]; [PMID: 19095996]

671 Turk V, Turk B, Guncar G, Turk D, Kos J. Lysosomal cathepsins: structure, role in antigen processing and presentation, and cancer. Adv Enzyme Regul 2002; 42: 285-303. [DOI: 10.1016/S00652571(01)00034-6]; [PMID: 12123721]

672 Turk V, Turk B, Turk D. Lysosomal cysteine proteases: facts and opportunities. EMBO J 2001; 20(17): 4629-4633. [DOI: 10.1093/ emboj/20.17.4629]; [PMID: 11532926]

673 Cheng XW, Huang ZH, Kuzuya M, Okumura K, Murohara T. Cysteine protease cathepsins in atherosclerosis-based vascular disease and its complications. Hypertension 2011; 58(6): 978-986. [DOI: 10.1016/HYPERTENSIONAHA.111.180935]; [PMID: 21986502]

674 Dollery CM, Libby P. Atherosclerosis and proteinase activation. Cardiovasc Res 2006; 69(3): 625-635. [DOI: 10.1016/ j.cardiores.2005.11.003]; [PMID: 16376322]

675 Qin Y, Shi GP. Cysteinyl cathepsins and mast cells proteases in the pathogenesis and therapeutics of cardiovascular diseases. Pharmacol Ther 2011; 131: 338-350.[DOI: 10.1016/ j.pharmthera.2011.04.010]; [PMID: 21605595]

676 Liu J, Sukhova GK, Yang JT, Sun J, Ma L, Ren A, et al. Cathepsin $\mathrm{L}$ expression and regulation in human abdominal aortic aneurysm, atherosclerosis, and vascular cells. Atherosclerosis 2006; 184(2): 
302-311. [DOI: 10.1016/j.atherosclerosis.2005.05.012]; [PMID: 15982660]

677 Lutgens SP, Cleutjens KB, Daeman MJ, Heeneman S. Cathepsin cysteine proteases in cardiovascular disease. FASEB $J$ 2007; 21(12): 3029-3041. [DOI: 10.1096/fj.06-7924com]; [PMID: 17522380]

678 Turk B, Turk D, Turk V. Lysosomal cysteine proteases: more than scavengers. Biochim Biophys Acta 2000; 1477(1-2): 98-111]; [PMID: 10708852]

679 Cheng XW, Kuzuya M, Nakamura K, Di Q, Liu Z, Sasaki T, et al. Localization of cysteine protease, cathepsin $\mathrm{S}$, to the surface of vascular smooth muscle cells by association with integrin alphanubeta3. Am J Pathol 2006; 168(2): 685-694. [DOI: 10.2352/ajpath.2006.050295]; [PMID: 16436681]

680 Liu J, Sukhova GK, Sun JS, Xu WH, Libby P, Shi GP. Lysosomal cysteine proteases in atherosclerosis. Arterioscler Thromb Vasc Biol 2004; 24(8): 1359-1366. [DOI: 10.1161/01. ATV.0000134530.27208.41]; [PMID: 15178558]

681 Doran AC, Meller N, McNamara CA. Role of smooth muscle cells in the initiation and early progression of atherosclerosis. Arterioscler Thromb Vasc Biol 2008; 28(5): 812-819. [DOI: 10.1161/ATVBAHA.107.159327]; [PMID: 18276911]

682 Shashkin P, Dragulev B, Ley K. Macrophage differentiation to foam cells. Curr Pharm Des 2005; 11(23): 3061-3072]; [PMID: 16178764]

683 Oörni K, Sneck M, Brömme D, Pentikäinen MO, Lindstedt KA, Mayranpaa M, et al. Cysteine protease cathepsin $\mathrm{F}$ is expressed in human atherosclerotic lesions, is secreted by cultured macrophages, and modifies low density lipoprotein particles in vitro. J Biol Chem 2004; 279(33): 34776-34784. [DOI: 10.1074/ jbc.M310814200]; [PMID: 15184381]

684 Mahmood DF, Jguirim-Souissi I, Khadija el-H, Blondeau N, Diderot V, Amrani S, et al. Peroxisome proliferators-activated receptor $\mathrm{g}$ induces apoptosis and inhibits autophagy of human monocyte-derived macrophages via induction of cathepsin L. Potential role in atherosclerosis. J Biol Chem 2011; 286(33): 28858-28866. [DOI: 10.1074/jbc.M111.273292]; [PMID: 21700710]

685 Kitamoto S, Sukhova GK, Sun J, Yang M, Libby P, Love $\mathrm{V}$, et al. Cathepsin L deficiency reduces diet-induced atherosclerosis in low-density lipoprotein receptor-knockout mice. Circulation 2007; 11(15)5: 2065-2075. [DOI: 10.1161/ CIRCULATIONAHA.107.688523]; [PMID: 17404153]

686 Kim DE, Kim JY, Schellingerhout D, Kim EJ, Kim HK, LeeS, et al. Protease imaging of human atheromata captures molecular information of atherosclerosis, complementing anatomic imaging. Arterioscler Thromb Vasc Biol 2010; 30(3): 449-456. [DOI: 10.1161/ATVBAHA.109.194613]; [PMID: 20056915]

687 Papaspyridonos M, Smith A, Burnand KG, Taylor P, Padayachee $\mathrm{S}$, Suckling KE, et al. Novel candidate genes in unstable areas of human atherosclerotic plaques. Arterioscler Thromb Vasc Biol 2006; 26(8): 1837-1844. [DOI: 10.1161/01. ATV.0000229695.6814.76]; [PMID: 16741146 688]

688 Jormsjö S, Wuttge DM, Sirsjö A, Whatling C, Hamsten A, Stemme $\mathrm{S}$, et al. Differential expression of cysteine and aspartic proteases during progression of atherosclerosis in apolipoprotein E-deficient mice. Am J Pathol 2002; 161(3): 939-945. [DOI: 10.1016/S00029440(10)64254-X]; [PMID: 12213722]

689 Li W, Dalen H, Eaton JW, Yuan XM. Apoptotic death of inflammatory cells In human atheroma. Arterioscler Thromb Vasc Biol 2001; 21(7): 1124-1130. [DOI: 10.1161/hq0701.092145]; [PMID: 11451740]

690 Reddy VY, Zhang QY, Weiss SJ. Pericellular mobilization of the tissue-destructive cysteine proteinases, cathepsin B, L, and S, by human monocyte-derived macrophages. Proc Natl Acad Sci USA 1995; 92(9): 3849-3853]; [PMID: 7731994]

691 Robert L, Robert AM, Jacotot B. Elastin-elastase-atherosclerosis revisited. Atherosclerosis 1998; 140(2): 281-295. [DOI: 10.1016/ S0021-9150(98)00171-3]; [PMID: 9862271]

692 Chwieralski CE, Welte T, Buhling F. Cathepsin-regulated apoptosis. Apoptosis 2006; 11(2): 143-149. [DOI: 10.1007/ s10495-006-3486-y]; [PMID: 16502253]

693 Frlan R, Gobec S. Inhibitors of cathepsin B. Curr Med Chem 2006; 13(19): 2309-2327]; [PMID: 16918357]

694 Gacko M, Chyczewski L, Chrostek L. Distribution, activity and concentration of cathepsin B and cystatin $\mathrm{C}$ in the wall of aortic aneurysm. Pol J Pathol 1999; 50(2): 83-86]; [PMID: 104811531]

695 Gacko M, Glowinski S. Cathepsin D and cathepsin L activities in aortic aneurysm wall and parietal thrombus. Clin Chem Lab Med 1998; 36(7): 449-52. 10.1515/CCLM.1998.075]; [PMID: 9746268]

696 Gacko M, Glowinski S. Activities of proteases in parietal thrombus of aortic aneurysm. Clin Chim Acta 1998; 271(2): 171-177. [DOI: 10.1016/S0009-8981(97)00246-5]; [PMID: 9565332]

697 Church LD, Cook GP, McDermott MF. Primer: inflammasomes and interleukin 1 beta in inflammatory disorders. Nat Clin Pract Rheumatol 2008; 4(1): 34-42. [DOI: 10.1038/ncprheum0681]; [PMID: 18172447]

698 Pham CT, Ley TJ. Dipeptidyl peptidase I is required for the processing and activation of granzymes $\mathrm{A}$ and $\mathrm{B}$ in vivo. Proc Natl Acad Sci USA 1999; 96(15): 8627-8632. [DOI: 10.1073/ pnas.96.15.8627]; [PMID: 10411926]

699 Mabee CL, McGuire MJ, Thiele DL. Dipeptidyl peptidase I and granzyme A are coordinately expressed during CD8+ T cell development and differentiation. J Immunol 1998; 160(12): 58805885]; [PMID: 9637500]

700 Dahl SW, Halkier T, Lauritzen C, Dolenc I, Pedersen J, Turk V, et al. Human recombinant pro-dipeptidyl peptidase I (cathepsin C) can be activated by cathepsin $\mathrm{L}$ and $\mathrm{S}$ but not by autocatalytic processing. Biochemistry 2001; 40(6): 1671-1678. [DOI: 10.1021/ bi001693z]; [PMID: 11327826]

701 Li W, Kornmark L, Jonasson L, Forssell C, Yuan XM. Cathepsin $\mathrm{L}$ is significantly associated with apoptosis and plaque destabilization in human atherosclerosis. Atherosclerosis 2009; 202(1): 92-102. [DOI: 10.1016/j.atherosclerosis.2008.03.027]; [PMID: 18495127]

702 Amuthan G, Biswas G, Zhang SY, Klein-Szanto A, Vijayasarathy C, Avadhani NG. Mitochondria-to-nucleus stress signaling induces phenotypic changes, tumor progression and cell invasion. $E M B O$ $J$ 2001; 20(8): 1910-1920. [DOI: 10.1093/emboj/20.8.1910]; [PMID: 11296224]

703 Wei DH, Jia XY, Liu YH, Guo FX, Tang ZH, Li XH, et al. Cathepsin L stimulates autophagy and inhibits apoptosis of oxLDL-induced endothelial cells: Potential role in atherosclerosis. Int J Mol Med 2013; 31(2): 400-406. [DOI: 10.3892/ ijmm.2012.1201]; [PMID: 23229094]

704 Cancel LM, Tarbell JM. The role of apoptosis in LDL transport through cultured endothelial cell monolayers. Atherosclerosis 2010; 208(2): 335-341. [DOI: 10.1016/ j.atherosclerosis.2009.07.051]; [PMID: 19709659]

705 Que XC, Ngo H, Lawton J, Gray M, Liu Q, Engel J, et al. The cathepsin B of Toxoplasma gondii, toxopain-1, is critical for parasite invasion and rhoptry protein processing. J Biol Chem 2002; 277(28): 25791-25797. [DOI: 10.1074/jbc.M202659200]; [PMID: 12000756]

706 Barret AJ, Kirschke H. Cathepsin B, cathepsin H, and cathepsin L. Methods Enzymol 1981; 80 Pt C: 535-561]; [PMID: 7043200]

707 Que XC, Engel JC, Ferguson D, Wunderlich A, Tomavo S, Reed SL. Cathepsin Cs are key for the intracellular survival of the protozoan parasite Toxoplasma gondii. J Biol Chem 2007; 282(7): 4994-5003. [DOI: 10.1074/jbc.M606764200]; [PMID: 17164247]

708 Dou Z, Carruthers VB. Cathepsin proteases in Toxoplasma gondii. Adv Exp Med Biol 2011; 712: 49-61. [DOI: 10.1007/978-1-44198414-2.4]; [PMID: 21660658] 
709 Que XC, Wunderlich A, Joiner KA, Reed SL. Toxopain-1 is critical for infection in a novel chicken embryo model of congenital toxoplasmosis. Infect Immun 2004; 72(5): 2915-2921. [DOI: 10.1128/IAI.72.5.2915-2921.2004]; [PMID: 15102804]

710 Huang R, Que X, Hirata K, Brinen LS, Li JH, et al. The cathepsin L of Toxoplasma gondii (TgCPL) and its endogenous macromolecular inhibitor, toxostatin. Mol Biochem Parasitol 2009; 164(1): 86-94. [DOI: 10.1016/j.molbiopara.2008.11.012]; [PMID: 19111576]

711 Larson ET, Parussini F, Huynh MH, Glebel JD, Kelley AM, Zhang $\mathrm{L}$, et al. Toxoplasma gondii cathepsin $\mathrm{L}$ is the primary target of the invasion-inhibitory compound morpholinurea-leucyl-homophenylvinyl sulfone phenyl. J Biol Chem 2009; 284: 26839-26850. [DOI: 10.1074/jbc.M109.003780]; [PMID: 19596863]

$712 \mathrm{Li} \mathrm{H}$, Child MA, Bogyo M. Proteases as regulators of pathogenesis: Examples from Apicomplexa. Biochim Biophys Acta 2012; 1824(1): 177-185. [DOI: 10.1016/j.bbapap.2011.06.002]; [PMID: 21683169]

713 Parrusini F, Coppens I, Shah PP, Diamond SL, Carruthers VB. Cathepsin L occupies a vacuolar compartment and is a protein maturase within the endo/exocyticsystem of Toxoplasma gondii. Mol Microbiol 2010; 76(6): 1340-1357. [DOI: 10.1111/j.13652958.2010.07181.x]; [PMID: 20444089]

714 Laliberte J, Carruthers VB. Toxoplasma gondii toxolysin 4 is an extensively processed putative metalloproteinase secreted from micronemes. Mol Biochem Parasitol 2011; 177(1): 49-56. [DOI: 10.1016/j.molbiopara.2011.01.009]; [PMID: 21277910]

715 Lagal V, Binder EM, Huynh MH, Kafsack BF, Harris PK, Diez $\mathrm{R}$, et al. Toxoplasma gondii protease TgSUB1 is required for cell surface processing of micronemal adhesive complexes and efficient adhesion of tachyzoites. Cell Microbiol 2010; 12(12): 1792-1808. [DOI: 10.1111/j.1462-5822.2010.01509.x]; [PMID: 20678172]

716 Miller SA, Thathy V, Ajioka JW, Blackman MJ, Kim K. TgSUB2 is a Toxoplasma gondii rhoptry organelle processing proteinase. Mol Microbiol 2001; 49(4): 883-894]; [PMID: 12890015]

717 Brossier F, Jewett TJ, Sibley LD, Urban S. A spatially localized rhomboid protease cleaves cell surface adhesions essential for invasion by Toxoplasma. Proc Natl Acad Sci USA 2005; 102(11): 4146-4151. [DOI: 10.1073/pnas.0407918102]; [PMID: 15753289]

718 Daneri-Navarro A, Del Toro-Arreola S, Sanchez-Hernandez PE, Ramirez-Duenas MG, Armendariz-Borunda J, PerezMonfort R. Immunosuppresive activity of proteases in cervical carcinoma. Gynecol Oncol 2005; 98(1): 111-117. [DOI: 10.1016/ j.ygyno.2005.03.034]; [PMID: 15894359]

719 Röcken C, Tautenhahn J, Bühling F, Sachwitz D, Vöckler S, Goette A, Bürger T. Prevalence and pathology of amyloid in atherosclerotic arteries. Arterioscler Thromb Vasc Biol 2006; 26: 676-677. [DOI: 10.1161/01.ATV.0000201939.10103.be]; [PMID: 16484604]

720 Cataldo AM, Nixon RA. Enzymatically active lysosomal proteases are associated with amyloid deposits in Alzheimer's brain. Proc Natl Acad Sci USA 1990; 87(10): 3861-3865]; [PMID: 1692625]

721 Dreyer RN, Bausch KM, Fracasso P, Hammond LJ, Wunderlich $\mathrm{D}$, Wirak DO, et al. Processing of the pre-b-amyloid protein by cathepsin D is enhanced by a familial Alzheimer's disease mutation. Eur J Biochem 1994; 224(2): 265-271. [DOI: 10.1111/ j.1432-1033.1994.00265.x]; [PMID: 7523115]

722 Hook V, Funkelstein L, Wegrzyn J, Bark S, Kindy M, Hook G. Cysteine cathepsins in the secretory vesicle produce active peptides: cathepsin $\mathrm{L}$ generates peptide neurotransmitters and cathepsin B produces beta-amyloid of Alzheimer's disease. Biochim Biophys Acta 2012; 1824(1): 89-104. [DOI: 10.1016/ j.bbapap.2011.08.015]; [PMID: 21925292]

723 Tian R, Hou G, Li D, Yuan T-F. A possible change process of inflammatory cytokines in the prolonged chronic stress and its ultimate implications for health. Sci World J 2014; 2014: 780616.
[DOI: 10.1155/2014/780616]; [PMID: 24995360]

724 Mehta JL, Saldeen TGP, Rand K. Interactive role of infection, inflammation and traditional risk factors in atherosclerosis and coronary artery disease. J Am Coll Cardiol 1998; 31(6): 1217 1225. [DOI: 10.1016/S0735-1097(98)00093-X]; [PMID: 9581711]

725 Werb Z, Foley R, Munck A. Interaction of glucocorticoids with macrophages - identification of glucocorticoid receptors in monocytes and macrophages. J Exp Med 1978; 147(6): 1684 1694. [DOI: 10.1084/jem.147.6.1684]; [PMID: 681878]

726 Rhinehart JJ, Balcerzak SP, Sagone AL, LoBuglio AF. Effect of corticosteroids on human monocyte function. J Clin Invest 1974; 54(6): 1337-1343. [DOI: 10.1172/JCI107880]; [PMID: 4612058]

727 Schreiber AD, Parsons J, McDermott P, Cooper RA. Effect of corticosteroids on the human monocyte IgG and complement receptors. J Clin Invest 1975; 56(5): 1189-1197. [DOI: 10.1172/ JCI108196]; [PMID: 1184745]

728 Zwet TL, Thompson J, Furth R. Effect of glucocorticosteroids on the phagocytosis and intracellular killing by peritoneal macrophages. Infect Immun 1975; 12(4): 699-705]; [PMID: 811557]

729 Werb Z. Biochemical actions of glucocorticoids on macrophages in culture. Specific inhibitions of elastase, collagenase, and plasminogen activatior secretion and effects on other metabolic functions. J Exp Med 1978; 147(6): 1695-1712. [DOI: 10.1084/ jem.147.6.1695]; [PMID: 210248]

730 Brattsand R, Linden M. Cytokine modulation by glucocorticoids: mechanism and actions in cellular studies. Aliment Pharmacol Ther 1996; 10(Suppl 2): 91-92. [DOI: 10.1046/j.13652036.1996.22164025.x]; [PMID: 8899106]

731 Saklatvala J. Glucocorticoids: do we known how they work? Arthritis Res Ther 2002; 4(3): 146-150. [DOI: 10.1186/ar398]; [PMID: 12010562]

732 De Bosscher K, Haegeman G. Latest perspectives on antiinflammatory actions of glucocorticoids. $\mathrm{J} \mathrm{Mol} \mathrm{Endocrinol}$ 2009; 23(3): 281-291. [DOI: 10.1210/me.2008-0283]; [PMID: 19095768]

733 Ehrchen J, Steinmüller L, Barczyk K, Tenbrock K, Nacken W, Eisenacher $\mathrm{M}$, et al. Glucocorticoids induce differentiation of a specifically activated, antiinflammatory subtype of human monocytes. Blood 2007; 109(3): 1265-1274. [DOI: 10.1182/ blood-2006-02-001115]; [PMID: 17018861]

734 Kalbak K. Incidence of arteriosclerosis in patients with rheumatoid arthritis receiving long-term corticosteroid therapy. Ann Rheum Dis 1972; 31(3): 196-200]; [PMID: 5032452]

735 Troxler RG, Sprague EA, Albanese RA, Fuchs R, Thompson AJ. The association of elevated plasma cortisol and early atherosclerosis as demonstrated by coronary angiography. Atherosclerosis 1977; 26(2): 151-162. [DOI: 10.1016/00219159(77)90098-3]; [PMID: 836352]

736 Klinkner AM, Waites CR, Kerns WD, Bugelski PJ. Evidence of foam cell and cholesterol crystal formation in macrophages incubated with oxidized LDL by fluorescence and electron microscopy. J Histochem Cytochem 1995; 43(10): 1071-1078. [DOI: 10.1177/43.10.7560885]; [PMID: 7560885]

737 Greenspan P, Yu H, Mao F, Gutman RL. Cholesterol deposition in macrophages: foam cell formation mediated by cholesterolenriched oxidized low density lipoprotein. J Lipid Res 1997; 38(1): 101-9]; [PMID: 9034204]

738 Glass CK, Witztum JL. Atherosclerosis. The road ahead. Cell 2001; 104(4): 503-516. [DOI: 10.1016/S0092-8674(01)00238-0]; [PMID: 11239408]

739 Libby P. Inflammation in atherosclerosis. Nature 2002; 420(6917): 868-874. [DOI: 10.1038/nature01323]; [PMID: 12490960]

740 Petrichenko IE, Daret D, Kolpakova GV, Shakhov YA, Larrue J. Glucocorticoids stimulate cholesteryl ester formation In human smooth muscle cells. Arterioscler Thromb Vasc Biol 1997; 17(6): 1143-1151. [DOI: 10.1161/01.ATV.17.6.1143]; [PMID: 9194766] 
741 Wang T, Gao JM, Yi SQ, Geng GQ, Gao XJ, Shen JL, et al. Toxoplasma gondii infection in the peritoneal macrophages of rats treated with glucocorticoids. Parasitol Res 2014; 113(1): 351-358. [DOI: 10.1007/s00436-013-3661-3]; [PMID: 24248630]

742 Costa FR, Mota CM, Santiago FM, Silva MV, Ferreira MD, Fonseca DM, et al. GITR activation positively regulates immune responses against Toxoplasma gondii. PLOS ONE 2016; 11(3): e0152622. [DOI: 10.1371/journal.pone.0152622]; [PMID: 27027302]

743 Nishikawa Y, Kawase O, Vielemeyer O, Suzuki H, Joiner KA, Xuan X, et al. Toxoplasma gondii infection induces apoptosis in noninfected macrophages, role of nitric oxide and other soluble factors. Parasite Immunol 2007; 29(7): 375-385. [DOI: 10.1111/ j.1365-3024.2007.00956.x]; [PMID: 17576367]

744 Seabra SH, Da Souza W, DaMatta RA. Toxoplasma gondii partially inhibits nitric oxide production of activated murine macrophages. Exp Parasitol 2002; 100(1): 62-70. [DOI: 10.1006/ expr.2001.4675]; [PMID: 11971655]

745 Jones LA, Anthony JP, Henriquez FL, Lyons RE, Nickdel MB, Carter KC, et al. Toll-like receptor-4-mediated macrophage activation is differentially regulated by progesterone via the glucocorticoid and progesterone receptors. Immunology 2008; 125(1): 59-69. [DOI: 10.1111/j.1365-2567.2008.02820.x]; [PMID: 18373668]

746 Korhonen R, Lahti A, Hämäläinen M, Kankaanranta H, Moilanen E. Dexamethasone inhibits inducible nitric-oxide synthase expression and nitric oxide production by destabilising mRNA in lipopolysaccharide-treated macrophages. Mol Pharmacol 2002; 62(3): 698-704]; [PMID: 12181447]

747 McKay LI, Cidlowski JA. Molecular control of immune/ inflammatory responses: interactions between nuclear factor-kB and steroid receptor signaling pathways. Endocr Rev 1999; 20(4): 435-459. [DOI: 10.1210/edrv.20.4.0375]; [PMID: 10453354]

748 Masur H, Murray HW, Jones TC. Effect of hydrocortisone on macrophage response to lymphokine. Infect Immun 1982; 35(2): 709-714]; [PMID: 7056582]

749 Chandrasekar PH, Momin F. Bone Marrow Transplant Team. Disseminated toxoplasmosis in marrow recipients: a report of three cases and a review of the literature. Bone Marrow Transplant 1997; 19(7): 685-689. [DOI: 10.1038/sj.bmt.1700736]; [PMID: 9156245]

750 Mele A, Paterson PJ, Prentice HG, Leoni P, Kibbler CC. Toxoplasmosis in bone marrow transplantation: a report of two cases and systematic review of the literature. Bone Marrow Transplant 2002; 29(8): 691-698. [DOI: 10.1038/sj.bmt.1703425]; [PMID: 12180115]

751 Patchell RA. Neurological complications of organ transplantation. Ann Neurol 1994; 36(5): 688-703. [DOI: 10.1002/ana.410360503]; [PMID: 7979215]

752 Penn I. Some problems with posttransplant limphoproliferative disease. Transplantation 2000; 79(5): 705-706]; [PMID: 10755511]

753 Stracciari A, Guarino M. Neuropsychiatric complications of liver transplantation. Metab Brain Dis 2001; 16: 3-11]; [PMID: 117260]

754 Marco S, Cecilia F, Patrizia B. Neurologic complications after solid organ transplantation. Transplant Int 2009; 22(3): 269-278. [DOI: 10.1111/j.1432-2277.2008.00780]; [PMID: 19076332]

755 Paya CV, Fung JJ, Nalesnik MA, Kieff E, Green M, Gores G, et al. EBV-induced post-transplant lymphoproliferative disorders. ASTS/ASTP EBV PTLD Task Force and the Mayo Clinic Organized International Consensus Development Meeting. Transplantation 1999; 68(10): 1517-1525]; [PMID: 10589949]

756 Morhun PJ, Weisz JM, Elias SJ, Holland GN. Recurrent ocular toxoplasmosis in patients treated with systemic corticosteroids. Retina 1996; 16(5): 383-387]; [PMID: 8912963]

757 Ahlbom E, Gogvadze V, Chen M, Celsi G, Ceccatelli S.
Prenatal exposure to high levels of glucocorticoids increases the susceptibility of cerebellar granule cells to oxidative stressinduced cells death. PNAS 2000; 97(26): 14726-14730. [DOI: 10.1073/pnas.260501697]; [PMID: 11114198]

758 LeWinn KZ, Stroud LR, Molnar BE, Ware JH, Koenen KC, Buka SL. Elevated maternal cortisol levels during pregnancy are associated with reduced childhood IQ. 2009; 38(6): 1700-1710. [DOI: 10.1093/ije/dyp200]; [PMID: 19423658]

759 Hulinska D, Sykora J, Zastera M. Effect of cortisone on Toxoplasma gondii infection studied by electron microscopy. Folia Parasitol (Praha) 1990; 37(3): 207-212]; [PMID: 2227703]

760 Prandota J. The importance of Toxoplasma gondii infection in disease presenting with headaches. Headaches and aseptic meningitis may be manifestations of the Jarisch-Herxheimer reaction. Int $J$ Neurosci 2009; 119(12): 2144-2182. [DOI: 10.3109/00207450903149217]; [PMID: 19916846]

761 Prandota J. Idiopathic intracranial hypertension may be caused by reactivation of latent cerebral toxoplasmosis. Effect of drugs and biologic substances. In: Gemma C (ed.), Neuroinflammation. Pathogenesis, Mechanisms and Management, Nova Science Publishers, New York, 2012; pp. 273-336.

762 Kassi E, Adamopoulos C, Basdra EK, Papavassiliou AG. Role of vitamin D in atherosclerosis. Circulation 2013; 128(23): 2517. 2531. [DOI: 10.1161/CIRCULATIONAHA.113.002654]; [PMID: 24297817]

763 Holick MF. Vitamin D deficiency. N Engl J Med 2007; 357(3): 266-281. [DOI: 10.1056/NEJMra070553]; [PMID: 17634462]

764 Szeto FL, Reardon CA, Yoon D, Wang Y, Wong KE, Chen Y, et al. Vitamin D receptor inhibits atherosclerosis in mice. Mol Endocrinol 2012; 26(7): 1091-1101. [DOI: 10.1210/me.20111329]; [PMID: 22638071

765 Weng S, Sprague JE, Oh J, Riek AE, Chin K, Garcia M, et al. Vitamin D deficiency induces high blood pressure and accelerates atherosclerosis in mice. PLoS ONE 2013; 8(1): e54625. [DOI: 10.1371/journal.pone.0054625]; [PMID: 23349943]

766 Li YC. Molecular mechanism of vitamin D in the cardiovascular system. J Investig Med 2011; 59(6): 868-871. [DOI: 10.2310/ JIM.0b013e31820ee448]; [PMID: 21307778]

767 Lai H, Fishman EK, Gerstenblith G, Brinker JA, Tong W, Bhatia $\mathrm{S}$, et al. Vitamin D deficiency is associated with significant coronary stenoses in asymptomatic African American chronic cocaine users. Int J Cardiol 2011; 158(2): 211-216. [DOI: 10.1016/j.ijcard.2011.01.032]; [PMID: 21295360]

768 De Boer IH, Kestenbaum B, Shoben AB, Michos ED, Sarnak MJ, Siskovick DS. 25-hydroxyvitamin D levels inversely associate with risk for developing coronary artery calcification. $J \mathrm{Am} \mathrm{Soc}$ Nephrol 2009; 20: 1805-1812. [DOI: 10.1681/ASN.2008111157]; [PMID: 19443637]

769 Oh J, Weng S, Felton SK, Bhandare S, Riek A, Butler B, et al. $1,25(\mathrm{OH} 2)$ vitamin $\mathrm{D}$ inhibits foam cell formation and suppresses macrophage cholesterol uptake in patients with type 2 diabetes mellitus. Circulation 2009; 120(8): 687-698. [DOI: 10.1161/ CIRCULATIONAHA.109.856070]; [PMID: 19887238]

770 Takeda M, Yamashita T, Sasaki N, Nakajima K, Kita T, Shinohara $\mathrm{M}$, et al. Oral administration of an active form of vitamin D (calcitriol) decreases atherosclerosis in mice by inducing regulatory $\mathrm{T}$ cells and immature dendritic cells with tolerogenic functions. Arterioscler Thromb Vasc Biol 2010; 30(12): 24952503. [DOI: 10.1161/ATVBAHA.110.215459]; [PMID: 20930170]

771 Vanhoutte PM. Endothelial dysfunction and atherosclerosis. Eur Heart J 1997; 18(Suppl E): E19-E29]; [PMID: 9402468]

772 Artaza JN, Norris KC. Vitamin D reduces the expression of collagen and key profibrotic factors by inducing an antifibrotic phenotype in mesenchymal multipotent cells. $J$ Endocrinol 2009; 200(2): 207-221. [DOI: 10.1677/JOE-08-0241]; [PMID: 19036760] 
773 Giovannucci E. Vitamin D and cardiovascular disease. Curr Atheroscler Rep 2009; 11(6): 456-461]; [PMID: 19852887]

774 Kendrick J, Targher G, Smits G, Chonchol M. 25-Hydroxyvitamin $\mathrm{D}$ deficiency is independently associated with cardiovascular disease in the Third National Health and Nutrition Examination Survey. Atherosclerosis 2009; 205(1): 255-260. [DOI: 10.1016/ j.atherosclerosis.2008.10.033]; [PMID: 19091317]

775 Toubi E, Shoenfeld Y. The role of vitamin D In regulating immune responses. Isr Med Assoc J 2010; 12(3): 174-175]; [PMID: 20684184]

776 Lemire JM, Archer DC, Beck L, Spiegelberg HL. Immunosuppressive actions of 1, 25-dihydroxyvitamin D3: preferential inhibition of Th1 functions. J Nutr 1995; 125(6 Suppl): 1704S-1708S. [PMID: 7782931]

777 van Etten E, Mathieu C. Immunoregulation by 1, 25-dihydroxyvitamin D3: basic concepts. J Steroid Biochem Mol Biol 2005; 97(1-2): 93-101. [DOI: 10.1016/j.jsbmb.2005.06.002]; [PMID: 16046118]

778 Adorini L, Penna G. Control of immune diseases by the vitamin D endocrine system. Nature Clin Pract Rheumat 2008; 4(8): 404412. [DOI: 10.1038/ncprheum0855]; [PMID: 18594491]

779 Veldman CM, Cantorna MT, DeLuca HF. Expression of 1,25-dihydroxyvitamin $\mathrm{D}(3)$ receptor in the immune system. Arch Biochem Biophys 2000; 374(2): 334-338. [DOI: 10.1006/ abbi,1999.1605]; [PMID: 10666315]

780 van Halteren AG, Tysma OM, van Etten E, Mathieu C, Roep BO. 1alpha, 25-dihydroxyvitamin D3 or analogue treated dendritic cells modulate human autoreactive $\mathrm{T}$ cells via the selective induction of apoptosis. $J$ Autoimmun 2004; 23(3): 233-239. [DOI: 10.1016/j.jaut.2004.06.004]; [PMID: 15501394]

781 Bhalla AK, Amento EP, Serog B, Glimher LH. 1,25-dihydroxyvitamin D3 inhibits antigen-induced $\mathrm{T}$ cell activation. J Immunol 1984; 133(4): 1748-1754]; [PMID: 6206136]

782 Chen S, Sims GP, Chen XX, Gu YY, Lipsky PE. Modulatory effects of 1, 25-dihydroxyvitamin D3 on human B cell differentiation. J Immunol 2007; 179(3): 1634-1647. [DOI: 10.4049/jimmunol.179.3.1634]; [PMID: 17641030]

783 Jirapongsananuruk O, Melamed I, Leung DY. Additive immunosuppressive effects of 1, 25-dihydroxyvitamin D3 and corticosteroids on TH1, but not TH2, responses. $J$ Allergy Clin Immunol 2000; 106(5): 981-985. [DOI: 10.1067/ mai.2000.110101]; [PMID: 11080724]

784 Boonstra A, Barrat FJ, Crain C, Heath VL, Savelkoul HF, O'Garra A. 1alpha,25-Dihydroxyvitamin d3 has a direct effect on naïve CD4(+) T cells to enhance the development of Th2 cells. J Immunol 2001; 167(9): 4974-4980. [DOI: 10.4049/ jimmunol.167.9.4974]; [PMID: 11673504]

785 O’Connel K, Kelly S, Kinsella K, Jordan S, Kenny O, Murphy $\mathrm{D}$, et al. Dose-related effects of vitamin $\mathrm{D}$ on immune responses in patients with clinically isolated syndrome and healthy control participants: study protocol for an exploratory randomized double-blind placebo-controlled trial. Trials 2013; 14: 272. [DOI: 10.1186/1745-6215-14-272]; [PMID: 23981773]

786 Pelajo CF, Lopez-Benitez J, Miller LC. Vitamin D and autoimmune rheumatic disorders. Autoimmune Rev 2010; 9(7): 507-510. [DOI: 10.1016/j.autrev.2010.02.011]; [PMID: 20146942]

787 Youssef DA, Miller CW, El-Abbassi AM, Cutchins DC, Cutchins C, Grant WB, et al. Antimicrobial implications of vitamin D. Dermatoendocrinol 2011; 3(4): 220-229. [DOI: 10.4161/ derm.3.4.15027]; [PMID: 22259647]

788 Zittermann A. Vitamin A in preventive medicine: are we ignoring the evidence? Br J Nutr 2003; 89(5): 552-572. [DOI: 10.1079/ BJN2003837]; [PMID: 12720576]

789 Shoenfeld N, Amital H, Shoenfeld Y. The effect of melanism and vitamin D synthesis on the incidence of autoimmune diseases. Nat Clin Pract Rheumatol 2009; 5(2): 99-105. [DOI: 10.1038/ ncprheum0989]; [PMID: 19182816]

790 Arnson Y, Amital H, Shoenfeld Y. Vitamin D and autoimmunity: new aetiological and therapeutic considerations. Ann Rheum Dis 2007; 66(9): 1137-1142. [DOI: 10.1136/ard.2007.069831]; [PMID: 17557889]

791 Orbach H, Zandman-Goddard G, Amital H, Barak V, Szekanecz $\mathrm{Z}$, Szucs G, et al. Novel biomarkers in autoimmune diseases: prolactin, ferritin, vitamin D, and TPA levels in autoimmune diseases. Ann NY Acad Sci 2007; 1109: 385-400. [DOI: 10.1196/ annals.1398.044]

792 Cutolo M. Vitamin D and autoimmune rheumatic diseases. Rheumatology (Oxford) 2009; 48(3): 210-212. [DOI: 10.1093/ rheumatology/ken394]; [PMID: 18930963]

793 Cantorna MT, Yu S, Bruce D. The paradoxical effects of vitamin D on type 1 mediated immunity. Mol Aspects Med 2008; 29(6): 369-375. [DOI: 10.1016/j.mam.2008.04.004]; [PMID: 18561994]

794 Cannell JJ, Hollis BW, Zasloff M, Heaney RP. Diagnosis and treatment of vitamin D deficiency. Expert Opin Pharmacother 2008; 9(1): 107-118. [DOI: 10.1517/14656566.9.1.107]; [PMID: 18076342]

795 van Etten E, Branisteanu DD, Verstuyf A, Waer M, Bouillon R, Mathieu C. Analogs of 1, 25-dihydroxyvitamin D3 as dosereducing agents for classical immunosuppressants. Transplantation 2000; 69(9): 1932-1942]; [PMID: 10830234]

796 Lemire J. 1, 25-Dihydroxyvitamin D3 - a hormone with immunomodulatory properties. Z Rheumatol 2000; 59(Suppl 1): 24-27]; [PMID: 10769431]

797 Yuk JM, Shin DM, Lee HM, Yang CS, Jin HS, Kim KK, et al. Vitamin D3 induces autophagy in human monocytes/macrophages via cathelicidin. Cell Host Microbe 2009; 6(3): 231-243. [DOI: 10.1016/j.chom.2009.08.004]; [PMID: 19748465]

798 Rockett KA, Brookes R, Udalova I, Vidal V, Hill AV, Kwiatkowski D. 1,25-Dihydroxyvitamin D3 induces nitric oxide synthase and suppresses growth of Mycobacterium tuberculosis in a human macrophage-like cell line. Infect Immun 1998; 66(11): $5314-$ 5321]; [PMID: 9784538]

799 Oswald IP, Wynn TA, Sher A, James SL. NO as an effector molecule of parasite killing: modulation of its synthesis by cytokines. Comp Biochem Physiol Pharmacol Toxicol Endocrinol 1994; 108(1): 11-18]; [PMID: 7520338]

800 Liu PT, Stenger S, Li H, Wenzel L, Tan BH, Krutzik SR, et al. Toll-like receptor triggering of a vitamin D-mediated human antimicrobial response. Science 2006; 311(5768): 1770-1773. [DOI: 10.1126/science.1123933]; [PMID: 16497887]

801 Mahmoudi MJ, Saboor-Yaraghi AA, Zabetian-Targhi F, Siassi F, Zarnani AH, et al. Vitamin A decreases cytotoxicity of oxidized low-density lipoprotein in patients with atherosclerosis. Immunol Invest 2016; 45(1): 52-62. [DOI: 10.3109/08820139.2015.1095208]; [PMID: 26700065]

802 Wang TT, Nestel FP, Bourdeau V, Nagai Q, Wang J, Liao J, et al. Cutting edge: 1,25-dihydroxyvitamin D3 is a direct inducer of antimicrobial peptide gene expression. J Immunol 2004; 173(5): 2909-2912. [DOI: 10.4049/jimmunol.173.5.2909]; [PMID: 15322146]

803 Gombart AF, Borregard N, Koeffler HP. Human cathelicidin antimicrobial peptide CAMP gene is a direct target of the vitamin $\mathrm{D}$ receptor and its strongly up-regulated in myeloid cells by 1,25-dihydroxyvitamin D3. FASEB J 2005; 19(9): 1067-1077. [DOI: 10.1096/fj.04-3284com]; [PMID: 15985530]

804 Fabri M, Stenger S, Shin DM, Yuk JM, Liu PT, Realegeno S, et al. Vitamin D is required for IFN- $\gamma$-mediated antimicrobial activity of human macrophages. Sci Transl Med 2011; 3: 104ra102. [DOI: 10.1126/scitranslmed.3003045]; [PMID: 21998409]

805 Lorente F, Fontan G, Jara P, Casas C, Garcia-Rodriguez MC, Ojeda JA. Defective neutrophil motility in hypovitaminosis D rickets. Acta Paediatr Scand 1976; 65(6): 695-699]; [PMID: 187003]

806 Cannell JJ, Vieth R, Umhau JC, Holick MF, Grant WB, Madronich 
S, et al. Epidemic influenza and vitamin D. Epidemiol Infect 2006; 134(6): 1129-1140. [DOI: 10.1017/S0950268806007175]; [PMID: 16959053]

807 Bikle DD. Vitamin D and the immune system: role in protection against bacterial infection. Curr Opin Nephrol Hypertens 2008; 17(4): 348-352. [DOI: 10.1097/MNH.0b013e3282ff64a3]; [PMID: 18660668]

808 Jeng L, Yamshchikov AV, Judd SE, Blumberg HM, Martin GS, Ziegler TR, et al. Alterations in vitamin D status and antimicrobial peptide levels in patients in the intensive care unit with sepsis. J Transl Med 2009; 7: 28. [DOI: 10.1186/1479-5876-728]; [PMID: 19389235]

809 Ghaffarifar F, Abdolah Pour M, Sharifi Z, Dalimi Asl A, AlKawaz E. The effect of vitamin D3 alone and mixed with IFN- $\gamma$ on tachyzoites of Toxoplasma gondii (RH strain) proliferation and nitric oxide (NO) production in infected macrophages of BALB/C mice. Iran J Parasitol 2010; 5(3): 48-56]; [PMID: 22347255]

810 Rajapakse R, Uring-Lambert B, Andarawewa KL, Rajapakse $\mathrm{RP}$, Abou-Bacar A, Marcellin L, et al. 1,25(OH)2D3 inhibits in vitro and in vivo intracellular growth of apicomplexan parasite Toxoplasma. Iran J Steroid Biochem Mol Biol 2007; 103(3-5): 811-814. [DOI: 10.1016/j.jsbmb.2006.12.058]; [PMID: 17270431]

811 Rajapakse R, Mousli M, Pfaff AW, Uring-Lambert B, Marcellin I, Bronner C, et al. 1,25-Dihydroxyvitamin D3 induces splenocyte apoptosis and enhances BALB/c mice sensitivity to toxoplasmosis. J Steroid Biochem Mol Biol 2005; 96(2): 179-185. [DOI: 10.1016/ j.jsbmb.2005.03.002]; [PMID: 15939587]

812 Tanabe K, Kimata I, Takada S. Multiplication of Toxoplasma gondii in maturing erythroid cells. J General Microbiol 1980; 118(1): 45-49]; [PMID: 7420056]

813 Milovanovic I, Vujanic M, Klun I, Bobic B, Nikolic A, Ivovic $\mathrm{V}$, et al. Toxoplasma gondii infection induces lipid metabolism alterations in the murine host. Mem Inst Oswaldo Cruz 2009; 104(2): 175-178. [DOI: 10.1590/S0074-02762009000200008]; [PMID: 19430640]

814 Lecca M, Saba L, Sanfilippo R, Pintus E, Cadoni M, Sancassiani F, et al. Quality of life in carotid atherosclerosis: the role of comorbid mood disorders. Clin Pract Epidemiol Mental Health 2016; 12: 1-8. [DOI: 10.2174/1745017901612010001]; [PMID: 27346995]

815 Duffy AR, Beckie TM, Brenner LA, Beckstead JW, Seyfang A, Postolache TT, et al. Relationship between Toxoplasma gondii and mood disturbances in women veterans. Mil Med 2015; 180(6): 621-625. [DOI: 10.7205/MILMED-D-14-00488]; [PMID: 26032378]

816 Sanfelice RA, da Silva SS, Bosqui LR, Miranda-Sapla MM, Barbosa BF, Silva RJ, et al. Privastatin and simvastatin inhibit the adhesion, replication and proliferation of Toxoplasma gondii $(\mathrm{RH}$ strain) in HeLa cells. Acta Trop 2016; Dec 21. [DOI: 10.1016/ j.actatropica.2016.12.006]; [PMID: 28012901]

817 Cortez E, Stumbo AC, Oliveira M, Bartosa HS, Carvalho L. Statins inhibit Toxoplasma gondii multiplication in macrophages in vitro. Int J Antimicrob Agents 2009; 33(2): 185-186. [DOI: 10.1016/ ijantimicaq.2008.07.026]; [PMID: 18996682]

818 Moreno SNJ, Li ZH. Anti-infectives targeting the isoproneoid pathway of Toxoplasma gondii. Expert Opin Ther Targets 2008; 12(3): 253-263. [DOI: 10.1517/14728222.12.3.253]; [PMID: 18260336]

819 Li ZH, Ramakrishnan S, Striepen B, Moreno SNJ. Toxoplasma gondii relies on both host and parasite isoprenoids and can be rendered sensitive to atorvastatin. PLoS Pathog 9(10): e1003665. [DOI: 10.1371/journal.ppat.1003665]

820 Montazeri M, Ebrahimzadeh MA, Ahmadpour E, Sharif M, Sarvi S, Daryani A. Evaluation of propranolol effect on experimental acute and chronic toxoplasmosis using quantitative PCR. Antimicrob Agents Chemother 2016; 60(12): 7128-7133. [DOI: 10.1128/AAC.01323-16]; [PMID: 27645234]

821 Montazeri M, Daryani A, Ebrahimzadeh M, Ahmadpour E, Sharif M, Sarvi S. Effect of propranolol alone and in combination with pyrimethamine on acute murine toxoplasmosis. Jundishapur $J$ Microbiol 2015; 8(9): e22572. [DOI: 10.5812/jjm.22572]; [PMID: 26487920]

822 Manuck SB, Kaplan JR, Adams MR, Clarkson TB. Effects of stress and the sympathetic nervous system on coronary artery atherosclerosis in the cynomolgus macaque. Am Heart $J$ 1988; 116(1 Pt 2): 328-333. [DOI: 10.1016/0002-8703(88)90110-X]; [PMID: 2899392]

823 Orekhov AN, Baldenkov GN, Tertov VV, Ryong LH, Kozlov SG, Lyakishev AA, et al. Cardiovascular drugs and atherosclerosis: effects of calcium antagonists, b-blockers, and nitrites on atherosclerotic characteristics of human aortic cells. J Cardiovasc Pharmacol 1988; 12(Suppl. 6): S66-S68]; [PMID: 2468911]

824 Prandota J. Increased generation of antibodies directed against brain proteins in patients with autism and their families may be caused by $T$. gondii infection. Maternal and fetal microchimerisms probably play an important role in these processes acting as a "Trojan horse" in dissemination of the parasite. In: Gemma C (ed.), Neuroinflammation. Pathogenesis, Mechanisms, and Management. New York: Nova Science Publishers 2012; pp. 447638. ISBN 978-1-61942-462-3

825 Hester J, Mullins J, Sa Q, Payne L, Mercier C, Cesbron-Delauw MF, Suzuki Y. Toxoplasma gondii antigens recognized by IgG antibodies differ between mice with and without active proliferation of tachyzoites in the brain during the chronic stage of infection. Infect Immun 2012; 80(10): 3611-3620. [DOI: 10.1128/ IAI.00604-12]; [PMID: 22851753]

826 Sternberger LA. The unlabelled antibody peroxidaseantiperoxidase (PAP) method. In: Immunocytochemistry, $2^{\text {nd }}$ edn., (Cohen S and McClusky RT, eds). John Wiley \& Sons, New York 1979; pp. 104-169.

827 Conley FK, Jenkins KA. Immunohistological study of the anatomic relationship of Toxoplasma antigens to the inflammatory response in the brains of mice chronically infected with Toxoplasma gondii. Infect Immun 1981; 31(3) 1184-1192]; [PMID: 7228401]

Peer reviewers: Annapaola Zito, Kalyani R 